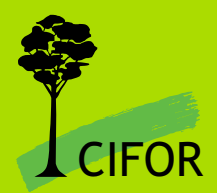

\title{
Faire progresser la REDD Enjeux, options et répercussions
}

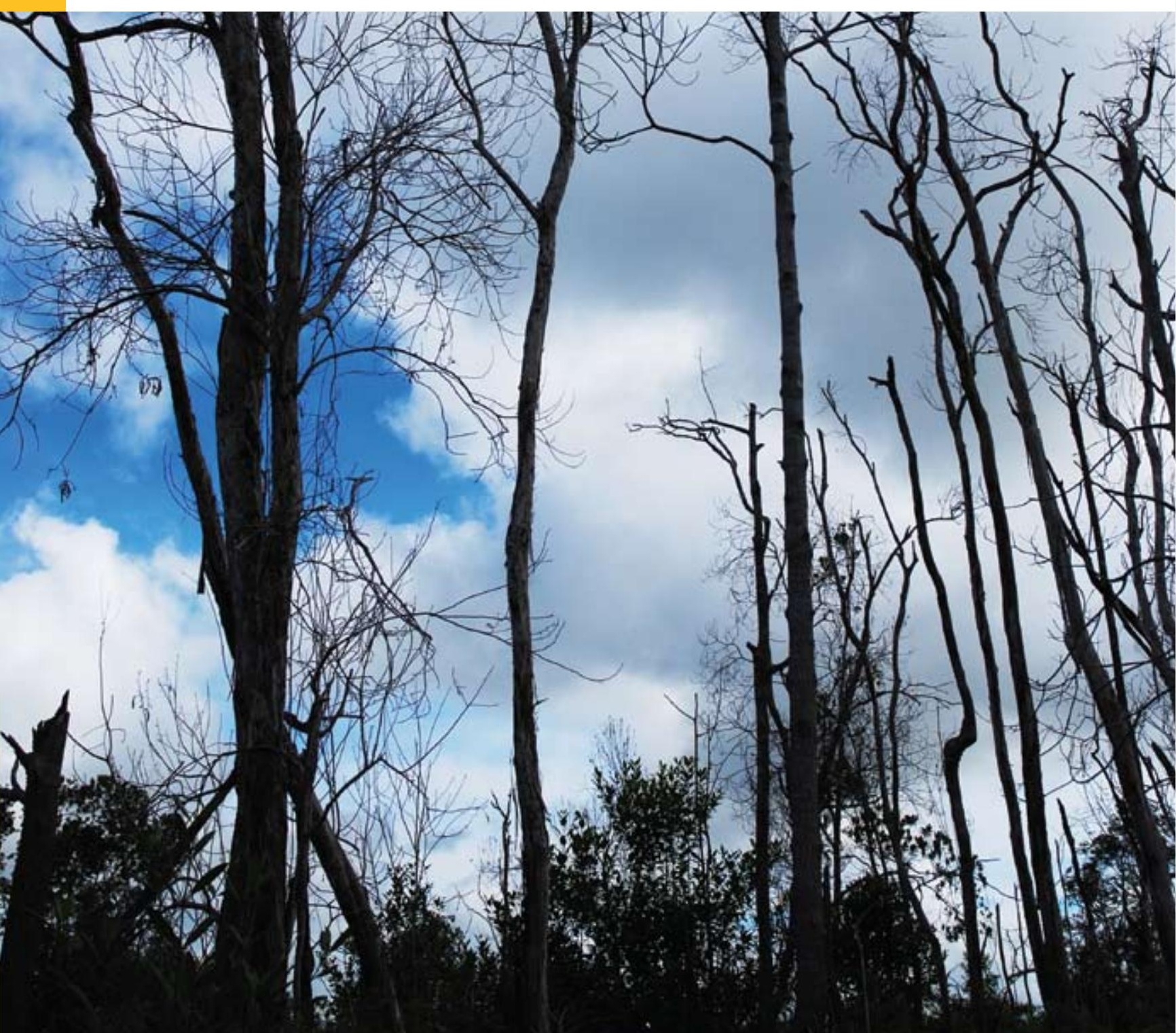





\section{Faire progresser la REDD Enjeux, options et répercussions}

Editeur : Arild Angelsen 


\section{Clause limitative de responsabilité}

Les opinions exprimées dans cet ouvrage sont celles des auteurs. Elles ne représentent pas nécessairement celles des institutions dont les auteurs font partie ni des personnes ou institutions ayant financé la publication du présent ouvrage.

Angelsen, A. (éd.) 2009 Faire progresser la REDD : Enjeux, options et répercussions. CIFOR, Bogor, Indonésie.

Photographes: couverture, chapitres 3, 7 \& 8 : Ryan Woo, chapitres 1 \& 4 : Brian Belcher, chapitre 2 : Herwasono Soedjito, chapitre 5 : Christophe Kuhn, chapitre 6 : Markku Kanninen, chapitre $9:$ Carol J.P. Colfer, chapitre 10 : Agung Prasetyo, chapitre 11 : Edmond Dounias.

Traduction de : Angelsen, A. (ed.) 2008 Moving ahead with REDD : Issues, options and implications. CIFOR, Bogor, Inodnesia.

Imprimé par SUBUR Printing, Indonésie

$188 \mathrm{p}$.

ISBN 978-602-8693-05-9

Publié par le Centre de recherche forestière internationale

JI. CIFOR, Situ Gede,

Bogor Barat 16115, Indonésie

Tel. : +62 (251) 8622-622 ; Fax : +62 (251) 8622-100

E-mail : cifor@cgiar.org

Site web : http ://www.cifor.cgiar.org

(c) par CIFOR

Tous droits réservés.

Publié en 2008

\section{Centre de recherche forestière internationale (CIFOR)}

CIFOR défend le bien-être humain, la conservation de l'environnement et l'équité en menant une recherche pour éclairer les politiques et les pratiques qui affectent les forêts dans les pays en développement. CIFOR est l'un des 15 centres au sein du Groupe consultatif sur la recherche agricole internationale (CGIAR). Le siège du CIFOR est situé à Bogor, en Indonésie. CIFOR a également des bureaux en Asie, en Afrique et en Amérique du Sud. 


\section{Table des matières}

Préface

iv

vi

viii

xii

1

5 Comment ajuster les besoins des pays aux sources de financement ? Michael Dutschke et Sheila Wertz-Kanounnikoff en collaboration avec Leo Peskett, Cecilia Luttrell, Charlotte Streck et Jessica Brown

6 Comment fixer les niveaux de référence pour les paiements au titre de la REDD ?

Arild Angelsen

7 Comment traiter les fuites ?

Sven Wunder

8 Comment garantir la permanence et déterminer les responsabilités ? Michael Dutschke en collaboration avec Arild Angelsen

9 Comment mesurer, surveiller et vérifier les émissions de $\mathrm{CO}_{2}$ liées aux forêts ?

Sheila Wertz-Kanounnikoff et Louis V. Verchot

en collaboration avec Markku Kanninen et Daniel Murdiyarso

10 Comment mesurer et surveiller la dégradation des forêts ?

Daniel Murdiyarso, Margaret Skutsch, Manuel Guariguata,

Markku Kanninen, Cecilia Luttrell, Pita Verweij et Osvaldo Stella Martins

11 Comment tirer parti des bénéfices associés à la REDD et éviter les effets nocifs ?

David Brown, Frances Seymour et Leo Peskett

Appendice : Récapitulatif des propositions présentées à la CCNUCC au titre de la REDD

Philippe Guizol et Stibniati Atmadja

Abréviations

Glossaire

Bibliographie 


\section{Préface}

Les émissions résultant de la déforestation et de la dégradation des forêts dans les pays en développement constituent environ 20 pour cent du total des émissions annuelles de gaz à effet de serre dans le monde. Ce type d'émissions n'est, à ce jour, pas inclus dans la Convention-cadre des Nations Unies sur les changements climatiques (CCNUCC) ni dans son Protocole de Kyoto.

Si nous voulons combattre le changement climatique et limiter la hausse de la température mondiale à deux degrés tout au plus, il faut impérativement inscrire à l'ordre du jour des prochains débats sur le climat la question de la réduction des émissions dues à la déforestation et à la dégradation des forêts (REDD) dans les pays en développement dans le cadre du prochain agenda pour le climat mondial.

La REDD peut générer d'autres bénéfices substantiels qui s'ajoutent à la réduction des émissions de gaz à effet de serre. Au nombre de ceux-ci figurent les effets positifs sur la biodiversité et le développement durable, notamment la réduction de la pauvreté et le renforcement des droits des peuples autochtones. Un programme REDD bien conçu est à même de rapporter un triple dividende - gains pour le climat, pour la biodiversité et pour le développement durable.

La Norvège a lancé une initiative internationale en faveur du climat et des forêts à la treizième Session de la Conférence des Parties à Bali en décembre 2007. A travers cette initiative, elle est prête à affecter aux activités de la REDD dans les pays en développement un montant de 3 milliards de couronnes norvégiennes par an au cours des cinq prochaines années. Les contributions de la Norvège, d'autres pays donateurs ainsi que celles provenant d'institutions multilatérales sont la manifestation incontestable de l'intérêt sincère et de l'engagement portés à la réduction des émissions résultant de la déforestation et de la dégradation des forêts dans les pays en développement.

Il ne sera néanmoins possible d'obtenir des réductions à grande échelle - des émissions de gaz à effet de serre résultant de la déforestation et de la dégradation des forêts dans les pays en développement - que si ces émissions sont incluses dans un agenda mondial climat post-2012. 
Certes, l'idée à la base du programme REDD est simple mais certaines questions y afférentes sont complexes comme celles liées à la détermination des mesures, des échelles, du financement, de la permanence, des responsabilités et de la fixation des niveaux de fuites de carbone et des niveaux de référence. Cet ouvrage a été publié avec le concours de la Norvège afin de faciliter et de faire progresser les négociations de la CCNUCC sur tous ces enjeux si complexes en précisant chacune des options y associées et, en particulier, leurs répercussions sur le plan d'une mise en application efficace, efficiente et équitable.

Porté par la ferme volonté de toutes les parties, le programme REDD pourrait être inclus dans le prochain accord sur le climat et rapporter un triple dividende. C'est là tout notre espoir et toute notre ambition.

Erik Solheim

Ministre de l'environnement et du développement international Norvège 


\section{Remerciements}

La rédaction et la publication de cet ouvrage n'ont pris que deux mois grâce à l'enthousiasme et au travail assidu de plus de trois douzaines de personnes.

Les auteurs ont travaillé des heures durant pour en rédiger le texte. Ils en ont tiré plusieurs avant-projets pour tenir compte des révisions apportées par des lecteurs internes et externes. La plupart des auteurs ont aussi fait une évaluation critique des chapitres autres que les leurs dans le cadre de la révision habituelle pratiquée par les pairs.

Quatre des chapitres de cet ouvrage (3, 4, 5 et 10) se fondent sur des documents et des fiches d'information (Infobriefs) préparés au titre d'un projet commun CIFOR-IPAM-ODI coordonné par Cecilia Luttrell. Les travaux ont progressé rapidement à l'instigation de Cecilia qui a méthodiquement compilé tous les documents et fait un travail de rédaction et de publication très complet pour ces quatre chapitres.

En charge de la coordination du processus de publication au siège du CIFOR à Bogor, Stibniati ("Nia ") Atmadja a relu et édité les chapitres et a contribué activement à plusieurs parties de cet ouvrage, notamment à l'inclusion des textes de la CCNUCC et du glossaire.

D'autres membres du personnel du CIFOR ont aussi pris une part active. Manuel Guariguata a présidé au recrutement des collaborateurs extérieurs chargés de faire l'évaluation critique tandis que Sandra McGuire a engagé toutes les forces vives du département de communication du CIFOR pour appuyer cette partie du processus. Gideon Suharyanto a veillé à ce que l'impression de l'ouvrage soit parfaitement conforme aux normes du CIFOR en la matière. La composition et le graphisme sont l'œuvre de Rahadian Danil tandis que Catur Wahyu l'a aidé à améliorer un grand nombre de figures.

Collaborateurs extérieurs, David Kaimowitz et Robert O’Sullivan ont relu le texte et apporté de judicieuses critiques et de précieuses suggestions. Outre les auteurs des chapitres, d'autres personnes ont fait l'évaluation critique d'un ou plusieurs chapitres ou ont contribué à leur rédaction : Laura Bozzi, Andrea Cattaneo, Joz Cozijnsen, Karsten Dunger, Manuel Estrada, Annette Frieberg, 
Alana George, Ole Hofstad, Dimitri Kanounnikoff, Katia Karousakis, Patrick van Laake, Michael Obersteiner, Krystof Obidzinski, Lucio Pedroni, Herry Purnomo, Paulo Moutinho, Stephan Schwartzmann, Fred Stolle et Dan Zarin. Sandra Child, Mark Havard, Guy Manners, Claire Miller, Henning Pape-Santos et Catharine Way ont jalousement veillé sur tout le processus de préparation avant la publication finale.

La réalisation de cet ouvrage a été financée par le gouvernement de la Norvège au titre de son initiative pour le climat et les forêts (International Climate and Forest Initiative). Le projet commun CIFOR-IPAM-ODI a été financé par la Fondation David et Lucile Packard (The David and Lucile Packard Foundation).

Ce livre est le résultat d'un solide travail d'équipe. A tous ceux et à toutes celles ainsi qu'à toutes les institutions qui ont apporté leur concours : terima kasib et tusen takk!

Bogor, Indonésie et Ås, Norvège, 30 novembre 2008

Arild Angelsen

Rédacteur de la publication 


\section{Résumé}

L'idée phare au cour du mécanisme de réduction des émissions dues à la déforestation et à la dégradation des forêts (REDD) est de récompenser les individus, les communautés, les projets et les pays qui réduisent les émissions de gaz à effet de serre (GES) liées aux forêts. Le programme REDD est en mesure de réduire en peu de temps et à faible coût les émissions de $\mathrm{CO}_{2}$ tout en contribuant en même temps à la réduction de la pauvreté et au développement durable.

Cela semble trop beau pour être vrai. Le programme REDD a pour fondement une idée simple qui plaît mais la traduire dans les faits est bien plus compliqué. Il nous faudra aborder bon nombre de questions ardues avant de pouvoir créer les mécanismes qui permettront d'exploiter pleinement le potentiel de la REDD. Comment mesurer la réduction des émissions quand les données sont insuffisantes, voire inexistantes ? Comment se procurer les milliards de dollars nécessaires pour mettre en place un mécanisme REDD ? Comment garantir les acquis de la réduction des émissions résultant de la déforestation et de la dégradation dans la réalité (gains) et veiller à ce qu'il n'en découle pas d'activités d'abattage dans d'autres zones forestières (fuites) ou l'année suivante (permanence) ? Comment faire participer les pauvres aux bénéfices qui en seront retirés ?

Toutes ces questions sont débattues dans cet ouvrage. Elles sont très opportunes pour structurer le programme REDD au moment même où se négocie l'agenda climatique mondial post-2012 au titre de la Convention-cadre des Nations Unies sur les changements climatiques (CCNUCC). Chaque chapitre décrit une question clé, en présente les options et en évalue les répercussions en fonction du critère des " $3 \mathrm{E}$ " : efficacité du carbone, efficience des coûts et équité des bénéfices y associés. Si des solutions techniques existent pour résoudre tous les problèmes, des compromis peuvent parfois être trouvés pour satisfaire à ces trois critères. Il y a aussi lieu de noter que les questions purement techniques sont rares et que la plupart des options ont des incidences politiques comme, par exemple, celle qui concerne la répartition des fonds du programme REDD dans les différents pays. 
Des niveaux de financement sans précédent seront nécessaires pour arriver à réduire les émissions dans des proportions, disons, de 50 pour cent. Il faudra avoir recours à un financement public, comprenant l'aide au développement, pour renforcer les capacités (capacité de réponse), mener à bien des exercices de démonstration, des réformes politiques et différentes activités dans des régions à haut risque et à faible gouvernance. L'exploitation du marché du carbone représente cependant une source potentielle de financements encore bien plus importante grâce à la vente directe de crédits carbone sur les marchés respectant les normes du programme REDD, ou aux fonds recueillis au titre de la mise aux enchères des permis d'émission ou d'une taxe imposée sur les échanges de carbone.

Certains craignent de voir se diluer ou se perdre les efforts engagés par ailleurs pour atténuer les effets du changement climatique si les crédits REDD (peu onéreux) deviennent des produits fongibles (interchangeables) avec d'autres types de crédits carbone. Ces craintes sont peut-être exagérées et diverses options ont été formulées pour réduire les risques au minimum. En particulier, si le programme REDD est inclus dans un accord mondial, les objectifs relatifs au total des émissions résultant des GES pourraient être plus ambitieux sans entraîner d'augmentation de coûts ; autrement dit, les négociations menées au titre de la CCNUCC ne doivent pas dissocier les décisions qui concernent les objectifs globaux des décisions qui portent sur l'inclusion de la REDD.

L'offre de crédits sera déterminante pour mesurer l'impact d'une intégration des crédits REDD dans le marché du carbone et sera elle-même influencée par les niveaux de référence des émissions. Au nombre des questions débattues dans le cadre de la REDD, l'argument le plus controversé est celui qui concerne les niveaux de référence. Ceux-ci ont en effet d'immenses répercussions sur l'application effective du programme comme sur un partage équitable entre les différents pays. Confrontés à un dilemme, les négociateurs doivent trouver le juste équilibre entre, d'une part, le risque de " réductions fictives " associé à des incitations édulcorées si les niveaux de référence sont trop généreux et, d'autre part, une faible participation au programme, voire son rejet, par les pays en développement si les niveaux de référence sont trop contraignants.

Pour que le mécanisme du programme REDD puisse fonctionner correctement, les réductions des émissions doivent être additionnelles, c'est-à-dire que les niveaux de référence des émissions ne doivent pas être fixés à un niveau supérieur à celui du scénario de la poursuite de la tendance actuelle (Business as usual, BAU). De plus, ces réductions doivent avoir un caractère permanent. Il sera aussi nécessaire de déterminer les responsabilités au cas où la permanence ne serait pas respectée si l'on veut assurer la fongibilité des crédits REDD qui pourront alors être échangés avec des crédits carbone d'autres secteurs.

Si la non-permanence est une forme de fuite dans le temps, il y aura fuite dans l'espace si le fait de réduire la déforestation et la dégradation dans une 
zone géographique donnée entraîne des taux d'émission plus élevés dans une autre. Sur un même territoire national, ces fuites peuvent être surveillées, voire éventuellement réduites, en redéfinissant les interventions, en neutralisant des activités ou en passant à l'échelle géographique supérieure de comptabilisation des crédits carbone.

Il existe trois grands niveaux d'approche pour déterminer l'échelle géographique de comptabilisation de la REDD et de l'accumulation des crédits de ce type sur le marché : le niveau sous-national, le niveau national, et un mélange des deux, ou approche combiné. L'approche nationale prend en compte les fuites survenant sur le territoire national et encourage les pays à s'engager dans la voie de réformes stratégiques de grande envergure (parfois peu coûteuses) qui peuvent aboutir à des réductions plus importantes et permanentes des émissions. Au niveau sous-national, l'approche peut prendre la forme de projets qui attireront les investisseurs privés parce que les résultats en sont plus tangibles; en outre, elle est susceptible de mieux réussir dans des pays qui ne sont institutionnellement pas prêts à adopter une approche nationale. Une approche combinée permettra aux pays de commencer par l'approche sous-nationale puis de passer progressivement à l'approche nationale, ou de comptabiliser et de recevoir simultanément des crédits aux niveaux sousnational et national. L'approche combinée est donc plus souple et permet à un plus grand nombre de pays de participer au programme REDD.

La capacité à surveiller, rendre compte et contrôler (Monitoring, Reporting and Verification, MRV) les émissions varie considérablement d'un pays à l'autre. Un programme REDD élaboré à l'échelle du monde doit être suffisamment souple pour éviter toute discrimination à l'encontre des pays insuffisamment dotés en capacités MRV. Au nombre des lignes directrices du groupe d'experts intergouvernemental sur l'évolution du climat (GIEC), par exemple, figure celle d'une plus grande tolérance à l'adhésion des pays au programme - leur permettant de se servir de valeurs globales par défaut pour les stocks de carbone par hectare lorsqu'ils comptabilisent leurs émissions. L'introduction de ce type de flexibilité permettrait de faire participer les pays pauvres qui ont des taux de dégradation élevés. Les incertitudes inhérentes à ces approches simplifiées signifient cependant qu'il faudrait " actualiser » les crédits carbones. Cela constituerait une incitation directe et encouragerait les pays à améliorer leurs méthodes de mesure et de surveillance pour finir par accéder aux crédits carbone sans réserve.

Les bénéfices associés au programme REDD sont potentiellement substantiels, notamment ceux qui sont liés à la réduction de la pauvreté, à l'amélioration de la gouvernance, à la conservation de la biodiversité et à la prestation d'autres services environnementaux. Si ce type de bénéfices dépend en grande partie des stratégies nationales en matière de REDD et de la façon dont les pays les mettent en œuvre (étude qui ne rentre pas dans le cadre de ce livre), l'architecture globale du programme REDD devrait permettre - et non interdire - aux 
pays en développement de saisir les opportunités d'adopter ce programme de façon à profiter de ces bénéfices sans entraîner d'effets dommageables. Les flux financiers du programme et sa mise en ouvre au niveau national doivent être harmonisés grâceà des engagements internationaux préexistants et à de nouvelles normes, en particulier des garanties de procédure, afin de minimiser le risque de conséquences négatives imprévues pour les communautés vulnérables.

Cet ouvrage met bien en évidence les différences qui existent d'un pays à l'autre à divers égards : infrastructure et dotations institutionnelles, capacités MRV pour mettre en œuvre les stratégies et mesures REDD, facteurs déterminants de la déforestation et de la dégradation des forêts, et ainsi de suite. Cette hétérogénéité doit être reflétée dans l'architecture globale du programme REDD dont les mécanismes doivent être suffisamment souples pour assurer la participation des pays la plus large fossible dès le début. Il faut veiller à prévoir en même temps les mécanismes qui permettront "d'avancer ", par exemple, pour améliorer les capacités MRV et passer d'une approche sous-nationale (projet) à une approche nationale. La flexibilité est nécessaire pour une autre raison également : la REDD est une expérience à grande échelle et il convient de prévoir une marge de manœuvre suffisante pour apporter les corrections nécessaires à mi-parcours de ce processus d'apprentissage en fonction de ce qui réussit ou non. 


\section{Liste des auteurs}

Arild Angelsen - Chercheur Associé, Centre de recherche forestière internationale (CIFOR), Indonésie et Professeur, Université Norvégienne des Sciences de la vie (UMB), Norvège - arild.angelsen@umb.no

Stibniati Atmadja - Chercheuse, Centre de recherche forestière internationale (CIFOR), Indonésie - s.atmadja@cgiar.org

David Brown - Chercheur, Overseas Development Institute (ODI), RoyaumeUni-d.brown@odi.org.uk

Jessica Brown - Chercheuse, Overseas Development Institute (ODI), Royaume-Uni-j.brown@odi.org.uk

Michael Dutschke - Directeur Exécutif, Biocarbon Consult, Allemagne michael@biocarbon.net

Manuel Guariguata - Chercheur, Centre de recherche forestière internationale (CIFOR), Indonésie-m.guariguata@cgiar.org

Philippe Guizol - Chercheur, Centre de coopération internationale en recherche agronomique pour le développement (CIRAD), France et Centre de recherche forestière internationale, Indonésie-p.guizol@cgiar.org

Markku Kanninen - Chercheur, Centre de recherche forestière internationale (CIFOR), Indonésie-m.kanninen@cgiar.org

Ruben Lubowski - Economiste, Environmental Defense Fund, Etats-Unis rlubowski@edf.org

Cecilia Luttrell - Chercheuse Associée, Centre de recherche forestière internationale (CIFOR), Indonésie - c.luttrell@cgiar.org

Daniel Murdiyarso - Chercheur, Centre de recherche forestière internationale (CIFOR), Indonésie - d.murdiyarso@cgiar.org

Leo Peskett - Chercheur, Overseas Development Institute (ODI), RoyaumeUni-l.peskett@odi.org.uk

Frances Seymour - Directrice Générale, Centre de recherche forestière internationale (CIFOR), Indonésie - f.seymour@cgiar.org

Margaret Skutsch - Chercheuse, Université de Twente, Pays-Bas - m.skutsch@ utwente.nl

Osvaldo Stella Martins - Coordinateur de Programme, Instituto de Pesquisa Ambiental da Amazônia (IPAM) - osvaldostella@ipam.org.br

Charlotte Streck - Directrice, Climate Focus, Pays-Bas - c.streck@climatefocus. com 
Louis Verchot - Chercheur, Centre de recherche forestière internationale (CIFOR), Indonésie - l.verchot@cgiar.org

Pita Verweij - Professeur Assistant, Université d'Utrecht, Pays-Bas p.a.verweij@uu.nl

Sheila Wertz-Kanounnikoff - Chercheuse Associée, Centre de recherche forestière internationale (CIFOR), Indonésie - s.wertz-kanounnikoff@cgiar. org

Sven Wunder - Chercheur, Centre de recherche forestière internationale (CIFOR), Indonésie - s.wunder@cgiar.org 

S'il est possible de contester certaines de ces affirmations qui sont décrites et analysées dans plusieurs chapitres de cet ouvrage, la plupart des observateurs reconnaissent toutefois que la REDD est un mécanisme clé des efforts déployés dans le monde pour limiter les effets du changement climatique. Nous devons faire progresser ce mécanisme. Dans les négociations internationales sur le climat, on entend par REDD toute une série d'actions et de démarches visant à réduire les émissions liées à la déforestation et à la dégradation des forêts. Au cœur même de ce dispositif se trouve le plan conceptuel qui confie à la communauté mondiale le soin de créer les mécanismes qui récompenseront ceux qui réduiront les émissions liées à la déforestation et à la dégradation des forêts - ce qui revient à puiser dans le potentiel de réduction des émissions de GES à travers une réduction "significative, expéditive, peu coûteuse et gagnant-gagnant » des émissions liées aux forêts.

La REDD s'articule donc autour d'une idée simple : payer ceux qui réduisent la déforestation et la dégradation des forêts. Comme c'est le cas pour toutes les idées simples cependant, la difficulté se trouve dans leurs mis-en-application. Parallèlement à l'enthousiasme, croît également une certaine inquiétude à propos de la faisabilité et des répercussions potentiellement négatives de la REDD. Il va falloir aborder de nombreuses questions difficiles si l'on veut créer un mécanisme réellement efficace : comment surveiller les réductions d'émissions, en rendre compte et les contrôler (Monitoring, Reporting and Verification, MRV) si les données relatives aux forêts sont de mauvaise qualité ou n'existent pas ? Comment financer la REDD compte tenu du fait qu'une réduction de $50 \%$ des émissions pourrait coûter de 20 à 30 milliards de dollars US par an ? Faut-il récompenser les projets, les pays, ou les deux à la fois ? Comment garantir le caractère permanent des réductions d'émissions autrement dit que les arbres épargnés une année ne seront pas abattus l'année suivante ? Comment éviter les fuites - à savoir que le fait d'épargner des arbres dans un pays ou une zone de projet n'entraine pas l'abattage d'un plus grand nombre d'arbres ailleurs? Comment s'assurer que toute réduction d'émission créditée est bien réelle; autrement dit qu'elle a un caractère additionnel à ce qui se passerait en l'absence d'un mécanisme REDD ? Comment veiller à ce que les paiements effectués au titre de la REDD soient équitablement distribués et bénéficient aux pauvres ? Toutes ces questions et d'autres encore doivent être débattues si nous voulons faire évoluer le mécanisme REDD et arriver à un accord sur ses modalités d'inclusion dans un agenda mondial de l'après 2012 pour le climat.

\subsection{Faire progresser la REDD}

La recette de ce livre est simple : nous posons les problèmes clé, présentons différentes solutions possibles et évaluons ensuite celles-ci sur la base du critère des " $3 \mathrm{E}$ » : Efficacité : le mécanisme peut-il entraîner une réduction 
substantielle des émissions ? Efficience : ces réductions sont-elles obtenues au moindre coût possible ? Équité : les coûts et bénéfices sont-ils répartis équitablement entre les pays et à l'intérieur d'un même pays ?

Le titre provisoire du livre était « REDD - architecture globale dans le cadre du nouvel agenda climatique ». Il met bien en évidence notre intention d'examiner différentes possibilités d'inclusion de la REDD dans l'accord mondial post2012 sur le climat actuellement négocié au sein de la Convention-cadre des Nations Unies sur les changements climatiques (CCNUCC). Nous étudions la conception et la mise en ouvre des projets REDD aux niveaux national et local seulement dans la mesure où ils relèvent de l'architecture globale du programme. Cela ne veut pas dire que les questions locales et nationales soient moins importantes pour la REDD mais plutôt qu'elles sont si complexes qu'elles justifieraient la parution d'un autre ouvrage qui leur serait spécifiquement consacré ! L'un des objectifs principaux de notre livre est de montrer que des options techniques existent pour tous les enjeux importants, même si des compromis sont parfois négociés entre les 3E. La complexité de la REDD, toutefois, ne tient pas seulement à ses aspects techniques mais au fait que les choix qui seront faits (les options) pourraient avoir de grandes répercussions sur la répartition des coûts et des bénéfices dans les différents pays. La question des données de base (niveaux de référence) constitue également un enjeu de taille à cet égard. Les niveaux de référence comportent en effet un aspect technique, à savoir la prévision réaliste des futurs taux de déforestation et de dégradation des forêts dans un scénario de la poursuite de la tendance actuelle (BAU) mais ils sont également déterminants pour fixer le niveau auquel un pays commencera à percevoir des crédits carbone au titre de la réduction de ses émissions, sur la base de l'interprétation de principes comme les " responsabilités communes mais différenciées » et les " circonstances nationales pertinentes ». Les niveaux de référence auront une influence importante sur les bénéfices et deviendront donc un enjeu politique.

Nombreux sont les articles de presse, rapports et déclarations qui sont publiés presque quotidiennement sur la REDD. Pourquoi écrire un livre sur le sujet? D'abord et avant tout, ce livre étudie toutes les grandes questions et options actuellement négociées au sein de la CCNUCC et, à notre connaissance, il n'existe actuellement pas d'autre livre sur ce sujet. Ensuite, il y a eu prolifération de plaidoyers en faveur de modèles REDD particuliers émanant aussi bien des parties de la CCNUCC (les pays) que des ONG environnementales, des organisations de recherche et des groupes de réflexion. Notre modeste ambition est de compléter ces offres et de présenter une évaluation des différentes options qui soit la plus objective possible. 


\subsection{Quel est l'objet de ce livre ?}

Reprenant les différentes options proposées pour structurer la REDD, ce livre les examine dans le contexte d'un agenda climatique mondial. Chaque chapitre porte sur une question que doivent nécessairement traiter aussi bien les négociateurs de la CCNUCC que tous les autres acteurs impliqués dans le débat mondial sur la REDD.

\section{Quelles sont les questions structurelles qui se posent en matière de REDD et} quels sont les critères qui permettent d'évaluer les options possibles? Trois grandes questions sont relevées à cet égard dans le chapitre 2. Premièrement, comment insérer la REDD dans l'architecture globale de la CCNUCC ? Doitelle faire partie d'un large accord mondial post-2012 sur le climat ou faire l'objet d'un accord séparé ? Les auteurs de ce livre relèvent que la réponse dépendra en grande partie de celle qui sera apportée à une autre question qui est celle du financement de la REDD. En effet, comment la REDD doit-elle être financée et - question subsidiaire - comment doit-elle être intégrée aux marchés du carbone ? Si les fonds de la REDD proviennent, par exemple, des marchés réglementaires (pour compenser les engagements des pays visés à l'Annexe I), il est alors préférable d'inclure la REDD dans un vaste accord post-2012.

Deuxièmement, la REDD doit-elle être incluse dans un cadre englobant le secteur forestier et, dans ce cas, la foresterie doit-elle aussi être incluse dans un système de comptabilisation élargi englobant l'agriculture, la foresterie et les autres utilisations des terres [(AFOLU) Agriculture, Forestry and Other Land Uses] ? Reste à résoudre la question-clé de la fusion ou non du programme de boisement/reboisement (B/R) - qui fait actuellement partie du Mécanisme de Développement Propre (MDP) au titre du Protocole de Kyoto - avec le mécanisme REDD dans un cadre de comptabilisation général englobant tout le secteur forestier.

Troisièmement, quelles mesures de performance utiliser ? Les stratégies et mesures permettant de réduire les émissions doivent-elles être comptabilisées (méthode des moyens) ou devons-nous plutôt mesurer les résultats (méthode des résultats) ? Si cette deuxième méthode est retenue, faudra-t-il lier les incitations aux niveaux absolus des stocks de carbone forestiers ou aux variations de ces stocks (équivalant à des émissions qui seront soit positives, soit négatives) ? La méthode basée sur les émissions est conforme à la conception d'ensemble actuelle et à l'architecture comptable de la CCNUCC, et constitue aussi le principal projet proposé au titre des négociations actuelles sur la REDD. C'est sur cette méthode qu'est essentiellement axé ce livre.

Quels sont les coûts et le potentiel de la REDD ? Trois grandes questions du débat sur la REDD sont abordées dans le chapitre 3. Quel sera le coût de la REDD ? Quels seront les effets de la REDD sur la stratégie globale visant à 
réduire les émissions de GES, sur le prix du carbone et les efforts pour réduire les émissions dans d'autres secteurs ? L'auteur de ce chapitre avance que la REDD offre une opportunité immédiate d'atténuer une source substantielle d'émissions à un coût relativement faible. Les coûts estimés pour réduire de moitié la déforestation varient de 7 à 28 milliards de dollars US par an, mais la comparaison avec d'autres options d'atténuation des émissions reste à l'avantage de la REDD même pour les estimations les plus élevées. Peu onéreuse et à même de réduire les émissions à brève échéance, l'exploitation du potentiel de la REDD pourrait entraîner une plus grande réduction des émissions pour un même coût global. Une étude a montré que l'inclusion de la REDD dans la stratégie globale pourrait réduire le réchauffement climatique de $0,25^{\circ} \mathrm{C}$ sans entraîner de surcoût.

Si les crédits carbone liés à la REDD deviennent fongibles et, par conséquent, interchangeables avec d'autres crédits carbone liés à la réduction des émissions de GES, certains craignent que les marchés du carbone soient inondés de crédits REDD bon marché et que cela supprime d'autres activités d'atténuation et freine le développement à long terme des technologies d'énergie propre. Quoiqu'il s'agisse d'une inquiétude certes légitime, certains auteurs comme Eliasch (2008) ont pu avancer qu'elle pourrait être surestimée. En outre, un certain nombre d'options tendent à minimiser ce risque, comme la fixation d'objectifs globaux plus restreints, l'imposition d'un contrôle sur la fongibilité et la " comptabilisation des crédits".

Quelle est la bonne échelle pour la REDD ? Le chapitre 4 analyse les trois grandes options envisagées pour comptabiliser la REDD et en créditer les bénéfices : (i) au niveau sous-national (ou à celui des projets) ; (ii) au niveau national ; ou (iii) à ces deux niveaux selon une approche combinée. S’ils adoptent l'approche combinée, les pays peuvent commencer par une approche sous-nationale et passer progressivement à l'approche nationale. Ils peuvent aussi comptabiliser et recevoir des crédits aux deux niveaux (sous-national et national) simultanément.

Dans le contexte d'une approche nationale, les gouvernements peuvent engager des réformes stratégiques de grande envergure (parfois peu coûteuses) veiller à la cohérence des rapports de surveillance et de contrôle (MRV) et rendre compte de toute "fuite de carbone " survenant sur le territoire national. Cette option favorise une meilleure intégration avec les politiques de développement national et une meilleure appropriation du mécanisme au niveau du pays. Elle est celle que préfèrent la plupart des pays. Les investisseurs privés sont plus favorables à une comptabilisation des crédits à l'échelle sous-nationale comme, par exemple, au niveau des projets, privilégiant éventuellement les projets forestiers à caractère tangible et de portée limitée et leurs liens plus directs avec les réductions d'émissions. Des projets peuvent être entrepris au titre de la REDD même quand le pays hôte n'est pas institutionnellement prêt à mettre 
en œuvre le mécanisme au niveau national. La troisième approche, approche combinée, est flexible et permet aux pays de combiner plusieurs mécanismes comptables et d'adopter différents rythmes d'application au niveau national. Elle permet donc de maximiser le potentiel des approches sous-nationale et nationale même s'il reste le défi d'harmoniser les deux niveaux en fin de parcours.

Comment ajuster les besoins des pays aux sources de financement ? Le chapitre 5 décrit les besoins de financement du programme REDD en fonction des trois stades d'intervention suivants : (i) les investissements initiaux nécessaires pour mettre en place l'infrastructure, les systèmes de surveillance des forêts, le renforcement des capacités et les autres activités préliminaires et de démonstration (capacité de réponse) ; (ii) les dépenses courantes de mise en œuvre des politiques et mesures nationales $(P M)$; et (iii) les paiements effectués aux propriétaires des forêts pour compenser leur manque à gagner (coûts d'opportunité).

L'aide publique au développement (APD) ainsi que d'autres formes de financement par l'état peuvent être source de financement pour les pays qui n'ont qu'un accès limité aux mécanismes globaux de la REDD. De bons exemples comprennent : des récompenses attribuées au titre d'actions anticipées et des crédits accordés pour mettre en œuvre les politiques et mesures nationales. Des mécanismes liés au marché comme l'inclusion de crédits REDD dans le marché du carbone, la mise aux enchères des permis d'émissions et/ou des taxes et redevances imposées sur les transactions de carbone sont les moyens les plus prometteurs pour collecter les fonds nécessaires à l'exploitation du plein potentiel de la REDD. Le financement pourrait cependant se révéler insuffisant (i) pendant la période de démonstration du mécanisme international de la REDD avant 2012 et (ii) dans les pays sans gouvernance forestière bien établie et dans lesquels, par voie de conséquence, le climat des investissements est à haut risque. Quel que soit le scénario, nous devons chercher à compenser le manque de financement de source publique comme de source privée. Il faut surtout garder présent à l'esprit que le futur mécanisme de la REDD doit être flexible et ouvert aux innovations en matière de financement afin de pouvoir s'adapter aux besoins changeants des pays et à leurs multiples expériences.

Comment fixer le niveau de référence des paiements effectués au titre de la $\boldsymbol{R E D D}$ ? Le chapitre 6 établit la distinction suivante entre trois significations du terme " niveaux de référence " dans le débat actuel : (i) les niveaux de référence historiques, autrement dit les taux de déforestation et de dégradation des forêts et les taux des émissions de GES en résultant au cours des $\mathrm{x}$ dernières années ; (ii) le scénario de la poursuite de la tendance actuelle (BAU), autrement dit l'évolution probable des émissions liées à la déforestation et à la dégradation en l'absence du mécanisme de la REDD, et (iii) les niveaux de référence des crédits comptabilisés, autrement dit le niveau auquel doivent 
démarrer les paiements qui seront effectués au titre de la REDD. Un niveau de référence - dans le contexte du scénario de la poursuite de la tendance actuelle (BAU) - est un étalon de mesure de l'impact des stratégies (conformes au principe d'additionnalité) adoptées au titre de la REDD tandis qu'un niveau de référence de crédit carbone sert d'étalon de mesure pour récompenser les pays (ou les projets) dont les taux d'émissions sont inférieurs à ce niveau. Si les niveaux de référence du scénario de la poursuite de la tendance actuelle (BAU) peuvent être considérés comme relevant du domaine technique, la fixation des niveaux de référence des crédits carbone dépend largement de la politique.

Presque toutes les propositions utilisent les niveaux de déforestation historiques comme point de départ et la plupart recommandent aussi de prendre en compte les " circonstances nationales " et de " récompenser les actions anticipées ». Il reste encore à mettre ces principes en pratique. Or les négociateurs se trouvent confrontés à un dilemme de taille. S’ils optent pour des niveaux de référence généreux, basés sur des évaluations par pays et tenant compte des circonstances nationales, ils risquent d'encourager les « réductions fictives » qui compromettrait l'intégrité environnementale (et, par conséquent, la réduction des émissions globales) et la crédibilité du mécanisme REDD ; mais s'ils imposent des niveaux de crédits carbone trop restreints ils risquent de rendre l'accord inacceptable. Il leur faut donc trouver un point d'équilibre délicat entre le risque de "réductions fictives " et l'acceptation politique du mécanisme REDD par les pays.

Comment traiter les fuites ? Le chapitre 7 aborde l'une des préoccupations-clé du débat sur la REDD. Comment garantir que la réduction de la déforestation et de la dégradation (DD) dans une zone géographique n'entraînera pas une plus grande DD et un taux d'émissions plus élevé dans une autre zone ? Les fuites causées par trois interactions de la forêt sur le climat sont aussi analysées dans ce chapitre, à savoir celles du boisement/reboisement (B/R), des zones de conservation protégées et de la gestion durable des forêts.

Il existe plusieurs façons de traiter la question des fuites de carbone ou «émissions déplacées ", notamment : (i) en surveillant ce qui se passe à l'extérieur des limites territoriales du projet ; (ii) en passant à l'échelle hiérarchiquement supérieure de la comptabilisation des crédits carbone, ce qui est l'un des principaux arguments en faveur d'une approche nationale plutôt que sous-nationale ; (iii) en escomptant les crédits basés sur des estimations de l'ampleur de la fuite ; (iv) en réorganisant les interventions pour réduire la fuite au minimum ; et (v) en neutralisant la fuite à travers des activités complémentaires, comme celles impliquant des « moyens de subsistance alternatifs ».

Les fuites peuvent donc être comptabilisées et les incitations (pour tenter de les maitriser) structurées pour en réduire l'ampleur. Elles sont toutefois l'une des composantes naturelles de la capacité d'adaptation d'une économie et ne 
peuvent être entièrement éradiquées ni justifier qu'un projet soit dénoncé. Considérées sous l'angle de l'équité et du développement, les fuites peuvent en fait être le signe d'une économie saine si, par exemple, en réponse à des obstacles dressés au titre de la REDD, il y a migration des facteurs de production vers de nouvelles opportunités et des pertes minimes pour les acquis sociaux (le bien-être). Reconnaître la possibilité de compromis entre l'atténuation des émissions de $\mathrm{CO}_{2}$ et des objectifs de développement plus larges peut donc nous amener à accepter délibérément l'éventualité des fuites et à revoir certaines de nos priorités en matière de mesures d'atténuation.

Comment garantir la permanence et déterminer les responsabilités ? Dans le chapitre 8 il est question d'une autre grande préoccupation du débat sur la REDD. Comment garantir qu'une zone forestière épargnée aujourd'hui ne sera pas détruite demain ? Qui devrait être tenu pour responsable si le cas se produit ? Quelles sont les dispositions à prévoir pour les contrats et les mécanismes financiers au titre de la REDD pour en garantir la permanence? Lorsque la responsabilité des stocks de carbone terrestre a pu être déterminée dans une zone géographique ou dans un secteur d'activités, il s'ensuit, non pas que le risque de non-permanence ait disparu mais, qu'il devra être compensé ailleurs. Cela pourrait être le cas à l'avenir si les pays en développement se fixent des objectifs d'émissions. En attendant, dans l'intervalle qui nous sépare de cette échéance, nous devons trouver des solutions intérimaires.

La gestion des risques de réémission est l'un des aspects permettant de garantir la permanence pour les projets comme pour les pays. Il faudra toutefois résoudre une autre question si nous voulons que les mécanismes REDD soient comptabilisés et rapportent des crédits carbones en conformité avec les marchés du carbone volontaires ou formels (réglementaires). La solution sera de mettre en place un système de responsabilité commerciale. Le chapitre 8 décrit les différents mécanismes nécessaires pour rendre les crédits carbones fongibles à partir de l'utilisation des terres et d'autres secteurs d'activités. Au nombre de ces mécanismes, les plus intéressants sont notamment les suivants : (i) des créditstampon pour les projets (inscription des crédits sur un compte temporaire); (ii) la mise en commun des risques pour un certain nombre de projets ; (iii) des garanties (assurance) commerciales ; et (iv) un partage des responsabilités sous forme de partenariats d'application des législations forestières (FCP) entre pays visés et pays non visés à l'Annexe 1 .

Comment surveiller, rendre compte et contrôler (MRV) les émissions de carbone liées aux forêts ? Le chapitre 9 examine les technologies existantes en matière de surveillance des forêts et analyse les avantages et inconvénients des différentes méthodes. Il existe deux méthodes de surveillance : (i) l'approche des différences de stocks, qui mesure les stocks de carbone forestier à différentes époques, et (ii) l'approche des entrées et des sorties, qui évalue le résultat net des flux dans le réservoir de carbone. 
Il existe un point d'équilibre entre le coût et la précision des méthodes. Dans les pays qui ont besoin d'un très grand degré de précision, ce sont des outils comme l'imagerie à fine résolution (pour discerner, par exemple, les signes de dégradation forestière ou de déboisement à petite échelle), l'imagerie répétée au fil du temps (pour surmonter, par exemple, les contraintes liées à la couverture nuageuse) ou encore l'imagerie de plus grande technicité associée à un traitement spécifique plus complexe (par exemple : pour analyser les images radar) qui seront utilisés et leur coût est loin d'être négligeable. De même, les techniques de mesures au sol, cruciales pour vérifier et mesurer les stocks de carbone, prennent du temps et sont relativement coûteuses à grande échelle, comme dans le cas de l'établissement d'un inventaire national.

Compte tenu de la grande variabilité des capacités de surveillance, rapports de suivi et de contrôle (MRV) des différents pays, il est nécessaire de prévoir pour la REDD un plan global qui soit suffisamment flexible pour éviter toute discrimination à l'encontre de pays n'ayant que de médiocres moyens dans ce domaine. Une approche progressive est recommandée afin de laisser les pays renforcer leurs capacités et acquérir de l'expérience avant de les intégrer éventuellement dans un mécanisme de paiement basé sur la performance, dans un futur agenda climatique. Il faudrait aussi prévoir des mesures susceptibles d'inciter tous les acteurs à davantage de précision et d'efficacité et appuyer le renforcement des capacités. Dans le but de surmonter les contraintes liées aux coûts et aux capacités nationales, est également examinée dans ce chapitre l'option consistant à mettre en place un système de surveillance centralisée et à en confier l'administration à une institution internationale.

Comment mesurer et surveiller la dégradation des forêts? Le chapitre 10 traite de la dégradation des forêts - beaucoup plus difficile à mesurer que la déforestation - mais qui est une source d'émission de GES trop importante pour ne pas être prise en compte. La dégradation des forêts peut être définie comme une réduction du taux de carbone par hectare de forêt (densité du carbone). Dans les cas où les données sont limitées, le groupe d'experts intergouvernemental sur l'évolution du climat (GIEC) suggère de commencer à calculer la dégradation à partir de niveaux relativement simples en surveillant les variations de superficies dans différents types de forêts mais en utilisant les valeurs par défaut globales des densités de $\mathrm{CO}_{2}$. Cela permettra d'arriver progressivement à une plus grande exactitude à mesure que l'on aura davantage de données nationales et sous-nationales. Les incertitudes inhérentes à ces approches simplifiées entraînent nécessairement des " actualisations " pour les crédits carbone - ce qui constituera une incitation directe pour les pays à améliorer leurs méthodes de mesure et de surveillance.

L'utilisation des méthodes préconisées par le GIEC (différences de stocks et évaluation des gains et pertes) et des graduations appropriées (niveaux de précision) devraient permettre de surmonter les difficultés liées à la 
comptabilisation du carbone dans le processus de dégradation des forêts qu'il serait dès lors réaliste d'inclure dans un accord sur la REDD. Le mécanisme REDD deviendrait ainsi d'autant plus efficace qu'il engloberait une plus large gamme d'émissions de gaz à effet de serre en provenance des forêts. L'équité en serait aussi améliorée sur le plan international parce qu'un plus grand nombre de pays, notamment en Afrique, seraient encouragés à participer à la REDD. Il importe donc que toute décision prise en matière de mesures MRV dans le contexte de la dégradation des forêts le soit en tenant compte des différences de circonstances.

Comment bénéficier des avantages associés à la REDD et éviter les effets dommageables?Le chapitre 11 s'intéresse aux raisons pour lesquelles la REDD a été l'objet d'une attention particulière pendant les négociations internationales sur le climat. La REDD a le potentiel de réduire la pauvreté, de protéger les droits humains, d'améliorer la gouvernance, de conserver la biodiversité et de procurer d'autres services environnementaux (par exemple des bénéfices associés) ainsi que de réduire les émissions des gaz à effet de serre. Toutefois elle peut aussi avoir des répercussions négatives sur les pauvres et les plus démunis de pouvoir si elle est appliquée en l'absence de garanties appropriées.

Ce chapitre relie chaque bénéfice associé à la REDD à des caractéristiques spécifiques aux niveaux international et national de façon à ce qu'il puisse être réalisé sans effets dommageables. Les auteurs estiment que : (i) il importe d'intégrer la REDD dans les grandes stratégies de développement économique pour que les avantages financiers qui en seront retirés bénéficient aux pauvres ; (ii) les paiements basés sur la performance, la transparence des données, la responsabilité financière et le contrôle international peuvent exercer une influence positive sur les droits humains et la gouvernance; et (iii) les bénéfices de la biodiversité peuvent être renforcés en ciblant des zones géographiquement vulnérables - même si les résultats dépendent aussi de facteurs externes telles que les stratégies et les politiques qui sont de véritables déterminants de la déforestation - et en encourageant ou en interdisant certains types d'activités.

Le défi que doit relever la communauté internationale est de veiller à ce que l'architecture globale préparée par la CCNUCC ouvre aux pays en développement des opportunités - loin de les en priver - afin qu'ils puissent mettre en œuvre le mécanisme de la REDD pour en retirer les bénéfices associés sans effets dommageables. Les bénéfices seront importants et les risques réduits s'ils sont associés à une harmonisation des flux financiers et de la mise en œuvre de la REDD au niveau national avec d'autres engagements internationaux préexistants, les normes émergentes - en particulier celles qui touchent au droit procédural - et les stratégies nationales de développement.

Plusieurs chapitres relèvent les circonstances très diverses que connaissent les différents pays en matière de capacités MRV, d'infrastructure institutionnelle 
pour mettre en œuvre la REDD, de facteurs déterminants de la déforestation et dela dégradation des forêts et ainsi de suite. Les stratégies nationales actuellement élaborées au titre de la REDD dans un certain nombre de pays reflètent bien cette diversité, mais il faut aussi que l'architecture globale de la REDD reflète cette hétérogénéité. Il n'y a pas de solution unique. Les mécanismes doivent être suffisamment flexibles pour assurer une large participation d'entrée de jeu. Ils devront en même temps être assortis d'incitations pour permettre « d'aller de l'avant ", par exemple, pour faire progresser les capacités MRV et passer d'une approche sous-nationale (projets) au niveau hiérarchiquement supérieur de l'approche nationale.

La flexibilité est nécessaire pour une autre raison encore : nous ne pouvons savoir avec certitude comment les mécanismes fonctionneront en pratique. Bien que ce livre tente d'évaluer quelles pourraient en être les répercussions sur différents modèles envisagés, la REDD reste une expérience à très grande échelle. "Les processus de négociations au niveau international sont souvent des exercices d'apprentissage à grande échelle à travers lesquels certaines parties $\mathrm{du}$ moins, modifient leur perception du problème, choisissent des politiques alternatives et voient aussi se transformer leurs incitations " (Underdal 2002 : 5). Notre but est de contribuer à ce processus d'apprentissage. 



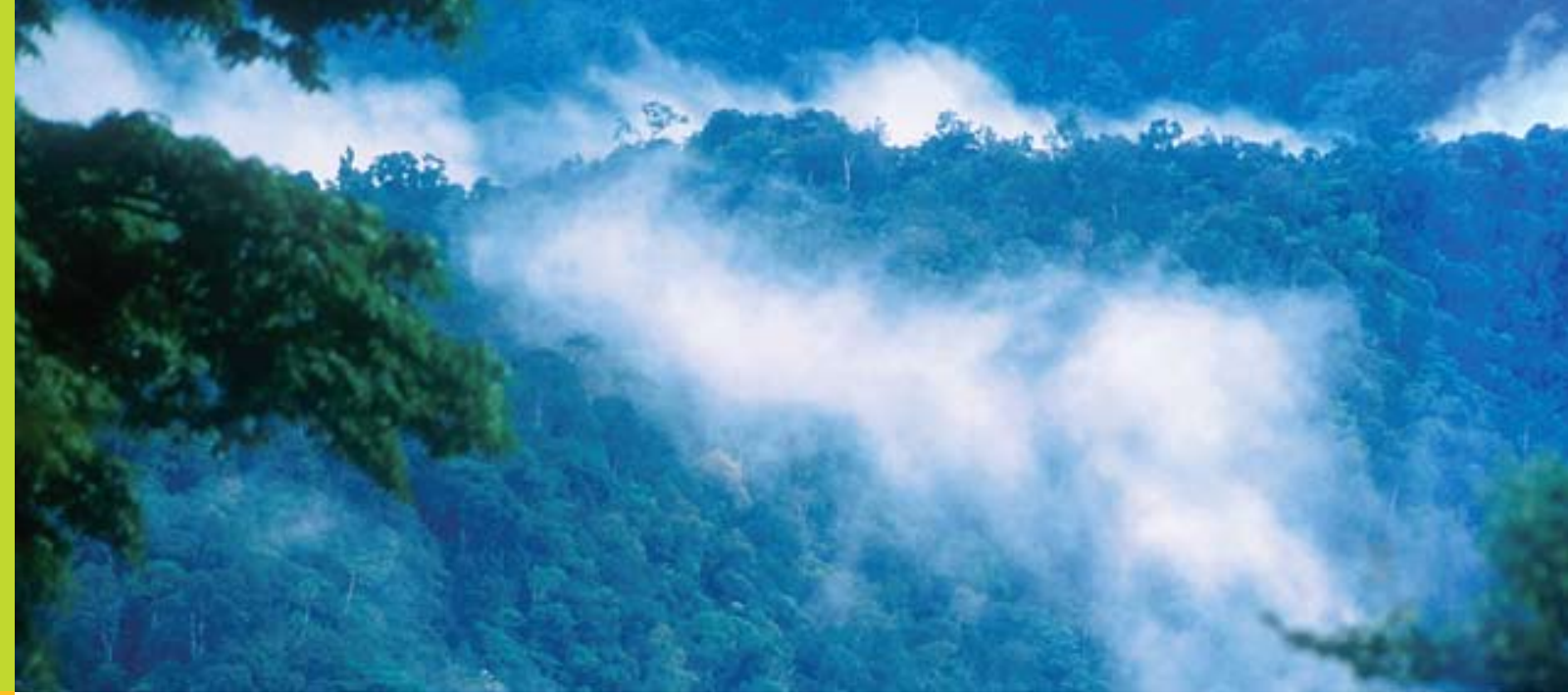

\section{Chapitre 2}

\section{Quels sont les enjeux clé du modèle REDD et les critères d'évaluation des options?}

Arild Angelsen et Sheila Wertz-Kanounnikoff

\subsection{Qu'est-ce que la REDD?}

La réduction des émissions liées à la déforestation et à la dégradation des forêts dans les pays en développement, ou REDD, fait partie des adjonctions les plus récentes au vocabulaire climatique. Au sens littéral de l'expression, la REDD est un objectif plutôt qu'une série clairement définie d'actions ou d'activités. La Convention-cadre des Nations Unies sur les changements climatiques (CCNUCC) se réfere à la REDD comme à un vaste ensemble d'approches et d'actions visant à réduire les émissions causées par la déforestation et la dégradation des forêts. ${ }^{1}$

Dans les débats, cependant, la REDD se réfère en premier lieu à : (i) l'élaboration de mécanismes de paiement pour faire des versements aux

Noter que l'utilisation de l'abréviation « REDD " n'est pas toujours uniforme dans le débat - y compris dans les documents de la CCNUCC - quant à l'inclusion explicite du terme " dégradation des forêts " dans le titre la treizième Conférence des Parties (COP 13) à Bali en 2007, par exemple, s'y réfère en tant que "réduction des émissions liées à la déforestation dans les pays en développement " (Décision 2/ CP.13), tandis que les soumissions les plus récentes concernant le Plan d'Action de Bali définissent la REDD comme la « réduction des émissions liées à la déforestation et à la dégradation des forêts dans les pays en développement » (FCCC/AWGLCA/2008/18). Toutes les explications mettent néanmoins toujours l'accent sur la réduction des émissions liées aux forêts dans les pays en développement. 
pays en développement au titre de la réduction de leurs émissions liées à la déforestation et à la dégradation des forêts (comparées à un niveau de référence); et (ii) des activités qui préparent les pays à participer au mécanisme de la REDD. Une question véritablement au cœur de la REDD est donc celle de la création d'un système de "paiements pour des services environnementaux à différents niveaux (international et national)» (PSE). Ce système de paiements à multiples niveaux est illustré dans la Figure 2.1.

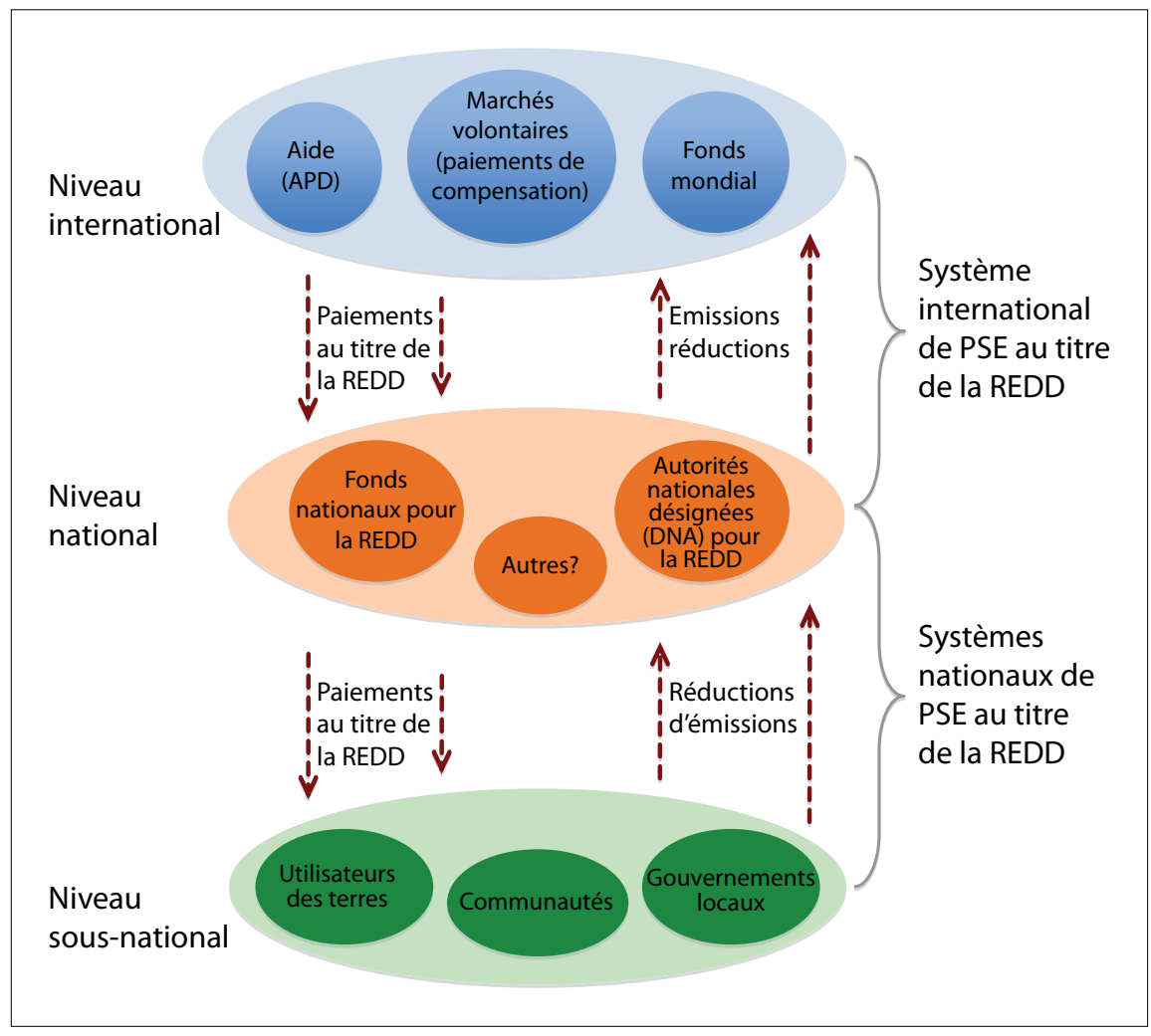

Figure 2.1. Modèle conceptuel d'un système REDD à multiples niveaux de " paiements pour des services environnementaux » (PSE)

Au niveau international, les acheteurs du service effectueront des paiements (en provenance, par exemple, des marchés volontaires ou réglementaires) aux prestataires de services (gouvernements ou entités sous-nationales dans les pays en développement) pour un service environnemental (moins d'émissions causées par la déforestation et la dégradation des forêts), ou l'adoption de mesures susceptibles de rendre ce même service (par exemple : les réformes foncières ou la mise en application des législations). Au niveau des pays, les gouvernements nationaux ou autres intermédiaires (les acheteurs du service) paieront les administrations ou les propriétaires fonciers au niveau sous-national (les prestataires de services) pour réduire les émissions, ou prendront d'autres 
mesures susceptibles de réduire les émissions (par exemple : la limitation de l'impact de l'abattage).

Les paiements directs du niveau international au niveau sous-national ne sont possibles que lorsque ces transactions sont approuvées par une institution gouvernementale nationale - par exemple, une autorité nationale désignée [Designated National Authority (DNA)] - comme c'est le cas pour le mécanisme de développement propre (MDP) et la mise en œuvre conjointe (MOC) [Joint Implementation (JI)] au titre du Protocole de Kyoto. Dans le débat actuel toutefois, l'approche sous-nationale n'est envisagée qu'en tant qu'étape intermédiaire d'un modèle national REDD (chapitre 4).

Il importe de noter que les stratégies nationales élaborées au titre de la REDD peuvent aussi inclure - outre des PSE - une large gamme de politiques comportant notamment des réformes foncières, une gestion plus efficace des zones forestières protégées et des politiques qui réduisent la demande de terres forestières et de leurs produits. L'un des avantages d'une approche nationale est précisément de pouvoir mettre en place des politiques à large base et de les comptabiliser dans la mesure où elles aboutissent à une réduction des émissions.

Dans ce livre nous mettons l'accent sur le niveau international - les options envisagées pour un accord global sur la REDD dans le cadre de la CCNUCC. Ce chapitre analyse quelques grandes questions - qui ne sont pas traitées dans les chapitres suivants - liées aux modèles conceptuels envisagés. Au nombre de ces questions figurent notamment les trois questions suivantes : (i) où situer la REDD dans l'architecture d'ensemble de la CCNUCC; (ii) le champ d'application de la REDD ; et (iii) la mesure de la performance. Enfin, nous présentons un ensemble de critères - le critère des $3 \mathrm{E}$ (ou triple E) - pour évaluer les options du modèle envisagé pour la REDD : efficacité (du carbone), efficience (des coûts) et équité (et son incidence sur les bénéfices associés). Ces critères sont utilisés tout au long des chapitres qui suivent.

\subsection{La REDD dans l'architecture d'ensemble de la CCNUCC}

L'une des questions cruciales débattues dans le contexte de la REDD est de savoir si ce mécanisme : (i) doit faire partie d'un agenda post-2012 élargi (cf. communications de la Coalition of Rainforest Nations, septembre 2007 et du Mexique, août 2008) ; ou (ii) faire l'objet d'un accord séparé (cf. Brésil, février 2007 et Center for Clean Air Policy (CCAP), août 2007). Si cette perspective peut sembler être, de l'avis de certains observateurs, une question purement technique, elle relève néanmoins d'enjeux fondamentaux débattus dans le cadre 
de la REDD dont le plus important est celui du financement de ce mécanisme. Si le financement provient (en partie) des marchés réglementaires, c'est à dire si les pays visés à l'Annexe 1 peuvent acheter des crédits REDD (paiements de compensation) en tant que faisant partie de leurs propres engagements, il est alors plus logique d'inclure la REDD dans un agenda post-2012 élargi. Si le financement doit provenir d'un apport de fonds, un accord séparé a probablement davantage de chances de réussite. Les positions adoptées dans ce débat représentent donc essentiellement des opinions divergentes quant au financement envisagé pour la REDD.

Un autre débat lié à celui du financement concerne celui de l'additionnalité de la REDD par rapport à des réductions obtenues dans d'autres secteurs. Il est intéressant de noter à cet égard que les deux écoles de pensée avancent les mêmes arguments. En général, ceux qui sont en faveur d'un accord REDD séparé pensent que cette séparation garantirait l'additionnalité en empêchant les crédits REDD bon marché " d'inonder " le marché et en ne déplaçant donc pas les efforts d'atténuation vers d'autres secteurs (voir chapitres 3, 4 et 6). Dans la droite ligne de cet argument, il s'ensuit que la meilleure façon de conférer l'additionnalité serait de maintenir la REDD à l'écart des autres engagements pris pour réduire les émissions de gaz à effet de serre (GES).

Ceux qui sont en faveur de l'inclusion de la REDD dans un agenda élargi post-2012 sur le climat tablent sur deux arguments principaux. Premièrement, la REDD a besoin d'être directement reliée aux marchés réglementaires (c.-à-d. qui vendent des crédits REDD en tant que paiements de compensation). Deuxièmement, l'intégration d'une opportunité d'atténuation à faible coût (c.-à-d. la REDD) dans un accord élargi permettra de fixer l'objectif global de réduction des émissions de GES à un niveau plus élevé sans frais supplémentaires (chapitre 3). Le système fonctionnera d'autant mieux que les objectifs de la REDD et les objectifs globaux de la réduction des émissions de GES seront négociés en même temps. Ceux qui sont en faveur de cette approche peuvent invoquer l'exemple du mécanisme pour un développement propre (MDP) dont les dispositions techniques ont été décidées à Marrakech en 2001 (COP 7) après que les objectifs globaux de réduction des émissions de GES aient été fixés à Kyoto en 1997 (COP 3). En conséquence, l'une des raisons pour lesquelles la déforestation évitée a été exclue du MDP tient précisément au fait qu'elle ne rapporte aucune réduction additionnelle des émissions.

Les questions relatives aux objectifs et aux engagements des pays en développement sont aussi au cœur des débats sur l'intégration de la REDD dans un accord élargi. D'aucuns envisagent pour l'avenir la mise en place d'un système complet de plafonnement et d'échange (CAT) qui inclue tous les pays et tous les secteurs (par exemple, Eliasch 2008). D'autres sont plus sceptiques et ne pensent pas que les pays en développement doivent adopter - du moins à brève échéance - des objectifs contraignants ; ils craignent en outre qu’inclure 
la REDD dans un accord général sur le climat puisse constituer le premier stade de l'élaboration d'un système CAT. La proposition selon laquelle les pays en développement auraient aussi besoin de réduire leurs émissions mais ne seraient pas liés par des objectifs contraignants tant que les pays développés n'auront pas montré le chemin en réduisant leurs taux d'émission de $\mathrm{CO}_{2}$ (Stern 2008) semble prometteuse et porteuse d'une initiative qui pourrait être reprise collectivement au niveau mondial pour atténuer les effets du changement climatique.

Il importe de se demander comment la REDD pourrait être intégrée dans le cadre de la CCNUCC étant donné l'incidence d'une telle intégration sur un grand nombre d'enjeux fondamentaux de ce mécanisme. Le degré de participation et le niveau d'engagement des parties en seront affectés (aussi bien du côté de l'offre que de la demande) ainsi que les modalités de financement de la REDD. Toute décision concernant l'architecture de la REDD ne devrait être prise qu'après la conclusion d'un accord sur toutes ces questions.

\subsection{Champ d'application de la REDD et activités d'atténuation comptabilisables}

Le débat sur le climat s'articule autour de la réduction de la concentration des gaz à effet de serre (GES) dans l'atmosphère. Inclure la totalité des puits et sources de carbone risque toutefois de devenir une tâche titanesque. Il serait préférable de considérer plutôt les négociations sur le climat comme le déroulement progressif d'actions conduites pas à pas où les mesures d'atténuation des effets du changement climatique font l'objet de discussions et sont peu à peu insérées dans un nombre croissant de secteurs et d'activités. L'une des questions clé de la REDD concerne le champ d'application des actions d'atténuation comptabilisables, deux grandes options pouvant être retenues à cet effet : (i) l'option consistant à inclure la REDD dans le secteur forestier ; et (ii) l'option visant à inclure la foresterie dans un cadre global avec l'agriculture, la foresterie et les autres utilisations des terres [agriculture, forestry and other land uses (AFOLU)]. Nous analyserons chacun de ces points en suivant.

Le stock total de carbone forestier est à tout moment déterminé par deux facteurs : la superficie totale de la forêt et la quantité de carbone par hectare de forêt (densité de carbone). Cela signifie que les variations peuvent être mesurées par ces deux facteurs : la superficie de la forêt et la densité de carbone. On peut, en outre, distinguer les activités qui atténuent les changements négatifs et celles qui renforcent les changements positifs. Il en résulte quatre façons différentes de promouvoir les stocks de carbone forestier comme le montre succinctement le Tableau 2.1. Il s'agit de la déforestation, du boisement/reboisement (B/R)], de la dégradation, et de la restauration/réhabilitation. 
Tableau 2.1. Champ possible des activités comptabilisables dans un mécanisme REDD foresterie. ${ }^{2}$

\begin{tabular}{lll}
\hline $\begin{array}{l}\text { Variations } \\
\text { modifiant : }\end{array}$ & $\begin{array}{l}\text { Atténuation des } \\
\text { changements négatifs }\end{array}$ & $\begin{array}{l}\text { Amélioration des } \\
\text { changements positifs }\end{array}$ \\
\hline $\begin{array}{l}\text { la superficie de la } \\
\text { forêt (hectare) }\end{array}$ & Déforestation éludée & Boisement et reboisement (B/R) \\
\hline $\begin{array}{l}\text { la densité de } \\
\text { carbone } \\
\text { (carbone par } \\
\text { hectare) }\end{array}$ & Dégradation éludée & $\begin{array}{l}\text { Restauration et réhabilitation de } \\
\text { la forêt (augmentation du stock } \\
\text { de carbone) }\end{array}$ \\
\hline
\end{tabular}

Le débat sur le champ d'application des activités comptabilisables dans le cadre de la REDD a sensiblement évolué au cours des trois dernières années. Au début, il s'agissait essentiellement de "limiter/atténuer les changements négatifs » entraînés par la déforestation (COP-11 en 2005 à Montréal) puis, par la dégradation des forêts (COP-13 en 2007 à Bali). A Bali, les Parties ont aussi convenues d'examiner les options visant à " augmenter les stocks de carbone forestier ", c'est-à-dire, de récompenser aussi éventuellement les " changements positifs renforcés" (Tableau 2.1) résultant d'actions de restauration/réhabilitation des forêts. ${ }^{3}$

Le processus d'augmentation des réserves de carbone peut être considéré comme le complément positif de celui de la dégradation des forêts - ce dernier faisant diminuer les densités de carbone alors que le premier les fait remonter. De même, les actions de boisement/reboisement $(B / R)$ peuvent être considérées comme le complément positif de la déforestation. Dans les deux cas, ce qui est au cœur de l'action engagée n'est pas seulement de stopper les changements négatifs (déforestation, dégradation des forêts), mais d'aller plus loin et de récompenser tout changement positif additionnel $(\mathrm{B} / \mathrm{R}$, augmentation des réserves de carbone).

Un argument logique déterminant pour inclure la REDD dans un système de comptabilisation cohérent - qui ne limiterait pas seulement les changements négatifs mais inciterait aussi aux changements positifs - tient au fait que les

\footnotetext{
Noter que même dans un système qui récompense les changements survenus dans une zone forestière, il faut néanmoins connaître les densités de carbone pour calculer la totalité des bénéfices liés au carbone. On peut supposer que les densités sont constantes dans le temps, ou les surveiller et les comptabiliser afin de déterminer les variations de quantités (émissions) de $\mathrm{CO}_{2}$ en provenance des forêts, comme on le fait pour les actions $\mathrm{B} / \mathrm{R}$ du MDP et pour certains projets du marché volontaire (voir chapitre 10).

Au paragraphe 11 de la Décision 2/CP.13 il est disposé ce qui suit : "Prend acte de la poursuite de l'examen, en application de la décision 1/CP.13, des démarches générales et des mesures d'incitation positives pour tout ce qui touche à la réduction des émissions résultant du déboisement et de la dégradation des forêts dans les pays en développement ainsi que du rôle de la conservation, de la gestion durable des forêts et des réserves de carbone des forêts dans les pays en développement ".
} 
mesures prises pour stopper les changements négatifs pourraient entraîner une restauration des terres forestières, c'est à dire une augmentation des densités de carbone. Pourquoi ne pas récompenser ces effets (augmentations) positifs ? Une logique similaire peut être appliquée aux augmentations de superficie. De manière explicite : une molécule de $\mathrm{CO}_{2}$ absorbée par l'atmosphère et stockée dans un arbre vaut tout autant que celle qui n'est pas émise.

L'un des défis de ce système global de comptabilisation forestière tient à ce que le boisement/reboisement $(\mathrm{B} / \mathrm{R})$ est déjà inclus dans le MDP au titre du Protocole de Kyoto. Cet argument pourrait suffire à exclure le volet $B / R$ $\mathrm{du}$ projet REDD. Deux solides contre-arguments peuvent toutefois y être immédiatement opposés. Le volet $\mathrm{B} / \mathrm{R}$ prévu au titre du MDP a, jusqu'ici et pour diverses raisons, été un échec. Si aucune révision importante dans les meilleurs délais n'est entreprise, exclure le volet B/R du nouvel accord REDD équivaudra à ne disposer d'aucun mécanisme efficace pour prendre en compte les changements positifs sur les surfaces forestières. De plus, exclure le volet $\mathrm{B} / \mathrm{R}$ de la REDD entraîne le risque d'aboutir à une fragmentation de toute l'architecture forestière envisagée (voir ci-dessous).

L'insertion de la REDD dans l'architecture de la CCNUCC soulève la question de déterminer la mesure dans laquelle la foresterie doit faire partie de systèmes de comptabilisation du carbone terrestre englobant l'agriculture, la foresterie et les autres utilisations des terres (AFOLU). Certes, il existe des arguments en faveur de cette approche AFOLU plus globale (Trines et al. 2006, Terrestrial Carbon Group 2008) qui assurerait un suivi cohérent des différents secteurs, activités et réserves de carbone entre toutes les parties. De nouvelles questions telles que la bioénergie pourraient aussi être traitées dans ce cadre plus global plutôt qu'un accord REDD distinct qui risquerait de fragmenter cette structure en systèmes séparés visant différentes catégories d'utilisation des terres. Il n'en reste pas moins, toutefois, que la mise en place d'un cadre AFOLU intégré est une tâche compliquée. Une solution prometteuse pourrait être de considérer la REDD comme l'une des pierres - pouvant être facilement imbriquée à l'avenir - de l'édifice AFOLU à construire.

\subsection{Approches basées sur les mesures ou sur les résultats composés d'émissions ou de stocks}

Une troisième question clé du modèle envisagé pour la REDD concerne la base de comptabilisation. Faut-il prendre pour base de référence les mesures nécessaires pour atteindre un résultat spécifique ou les résultats eux-mêmes? Ces deux approches sont qualifiées d'approche basée sur les mesures pour l'une et sur les résultats pour l'autre. 
Dans les modèles basés sur les mesures, les paiements sont soumis à des conditions imposées au titre des mesures supposées produire un résultat qualifié de souhaitable mais impossible à mesurer directement. Ces modèles sont aussi parfois désignés sous l'appellation de "politiques et mesures" (PM). Dans le contexte de la REDD, on trouve au nombre des PM, par exemple, les réformes foncières, la mise en application des législations forestières et l'adoption de pratiques d'utilisation des terres visant à garantir un résultat donné (comme, par exemple, la limitation de l'impact de l'abattage).

Dans les modèles basés sur les résultats, ce sont ces derniers qui conditionnent les paiements. Deux types de quantifications des résultats relèvent du débat sur la REDD : celles basées sur les émissions et celles basées sur les stocks. Dans la première approche, celles des émissions (ou des flux), seules sont prises en compte les variations nettes des stocks de carbone pendant des périodes spécifiques pour calculer les crédits carbone (voir chapitre 9). Dans une approche basée sur les stocks, les paiements sont déterminés en fonction du stock total de carbone dans une forêt donnée pendant une période spécifique, c'est-à-dire en fonction des niveaux absolus et non des variations (émissions).

Considérés sous l'angle de l'efficacité et de l'efficience, les modèles basés sur les résultats peuvent être préférés à ceux qui sont basés sur les mesures au motif qu'ils relient directement les paiements au service rendu. Il ne faudrait cependant pas oublier que le résultat doit être mesurable - condition nécessaire à laquelle il n'est pas toujours possible de satisfaire. Dans certaines situations, ni la gouvernance ni les institutions ne sont encore suffisamment étoffées pour garantir le fonctionnement d'approches basées sur les résultats. Dans d'autres situations, les méthodes qui sont pratiquées pourraient gêner ces approches, comme dans le cas de la dégradation des forêts (voir chapitre 10).

C'est une approche basée sur les émissions qu'a retenu le Protocole de Kyoto, rendant naturelle l'application de cette même démarche à la REDD. S’il est vrai que le terme "émissions » est enchâssé au cœur même de l'acronyme REDD, il n'empêche que les partisans d'une approche basée sur les stocks défendent leur point de vue en arguant que cette approche assurerait probablement une plus grande efficacité du carbone (Woods Hole Research Center (WHRC) et Amazon Institute for Environmental Research (IPAM) 2008, Terrestrial Carbon Group 2008) et serait mieux perçue par le secteur privé qui investira donc davantage (Centre for International Sustainable Development Law (CISDL) et Global Public Policy Institute (GPPI) 2007). Ces mêmes partisans d'une approche basée sur les stocks évoquent aussi les défis méthodologiques associés aux approches basées sur les émissions (notamment en ce qui concerne les niveaux de référence, la maîtrise des fuites et l'équité) qui peinent à prendre en compte les efforts préalablement déployés par les pays en matière de conservation de leurs forêts. 
Bien qu'une approche basée sur les stocks puisse permettre d'éviter certains des écueils d'une approche basée sur les émissions, cette dernière méthode présente néanmoins des avantages sur le plan de l'efficacité. Les transactions des marchés mondiaux émergents du carbone se font en fonction des réductions d'émissions. La conservation du stock ne permet pas, en soi, de générer des crédits d'émissions - ce qui supprime la possibilité d'aller puiser directement dans les marchés réglementaires pour financer les activités engagées au titre de la REDD. ${ }^{4}$

Un principe général pour disposer d'un mécanisme efficace consiste à cibler la difficulté aussi directement que possible. L'approche basée sur les émissions est une meilleure adepte de ce principe que les approches basées sur les stocks ou sur les mesures. Il y a aussi lieu de noter que l'approche basée sur les stocks comporte un risque important - celui que de gros paiements puissent être effectués au titre de zones forestières qui ne sont pas menacées et "diluent " ainsi les fonds disponibles qui ciblent les forêts menacées en ne rapportant qu'une faible additionnalité.

L'approche basée sur les émissions est conforme aux priorités actuelles et à l'architecture de la CCNUCC et constitue également la principale proposition qui a été présentée au titre des négociations sur la REDD. Ce sera donc cette approche - l'approche basée sur les émissions - dont il sera question dans le reste de cet ouvrage.

\subsection{Les critères d'évaluation des options ou critère des $3 \mathrm{E}$}

Un grand nombre de projets conceptuels ont été présentés au titre de la REDD. Comment les évaluer?

Un projet type de modèle conceptuel REDD cible la réduction d'émissions de GES au moindre coût possible, tout en contribuant au développement durable. Les projets de modèles (ou propositions) peuvent être évalués en fonction de cet objectif selon trois séries de critères qualifiés de "critère des $3 \mathrm{E}$ »: le mécanisme mis en place réussira-t-il à atteindre les objectifs fixés en matière d'émissions liées aux GES (efficacité) ? Sera-t-il possible d'atteindre cet objectif au moindre coût (efficience) ? Quelles en sont les incidences sur la répartition et sur les bénéfices y associés (équité et bénéfices y associés) ?

\footnotetext{
4 Des liens indirects peuvent être créés, par ex. en mettant aux enchères des quotas d'émissions de GES ou par des taxes sur les marchés du carbone pour financer des approches basées sur les stocks (voir chapitre 5).
} 


\subsubsection{Efficacité}

Par efficacité on entend "l'efficacité du carbone ", l'efficacité se référant dans ce contexte à l'ampleur de la réduction des émissions. Elle dépendra d'un certain nombre de facteurs, notamment de la faisabilité politique et du degré d'engagement des pays à participer et à mettre en œuvre les mécanismes de la REDD mais aussi - et c'est un aspect plus important encore - du modèle qui sera finalement retenu pour l'architecture de la REDD. Pour pouvoir réduire les émissions dans les plus fortes proportions possibles, le modèle REDD doit être doté d'un certain nombre de caractéristiques : étendu (réductions substantielles) et ampleur (englober toutes les sources et tous les puits d'importance significative), ainsi que flexibilité et robustesse pour tenir compte de la diversité des conditions locales.

Les réductions d'émissions ne sont pas directement observables mais peuvent être définies comme la différence existant entre les émissions résultant d'un scénario sans mécanisme REDD et les émissions résultant d'un scénario comportant l'application d'un mécanisme REDD. Il faudra mettre en place un système qui devra : (i) mesurer avec exactitude des émissions réelles et vérifiables liées à un scénario appliquant le mécanisme REDD ; et (ii) comporter des hypothèses réalistes de ce qui se passerait en l'absence d'application d'un mécanisme REDD (additionnalité). Enfin, il ne faut pas oublier que des actions engagées dans le contexte de la REDD peuvent avoir des effets secondaires indésirables dans l'espace (émissions déplacées ou fuites), dans le temps (permanence), et/ ou pour d'autres activités d'atténuation. Ces effets secondaires devront être pris en compte dans l'évaluation de l'efficacité globale. Les différents éléments constituant le critère de l'efficacité sont énumérés au Tableau 2.2.

Tableau 2.2. Eléments constitutifs du critère de l'efficacité

\section{Critère de l'efficacité}

Degré de

réduction et

additionnalité

Ampleur /

champ

d'application

Flexibilité et robustesse
Réduction des émissions en termes absolus ou relatifs comparée à celles d'un scénario de la poursuite de la tendance actuelle (BAU). L'additionnalité est un critère plus spécifique qui pose comme condition que les réductions soient additionnelles; autrement dit, elles s'ajouteraient à ce qui se passerait en l'absence d'un mécanisme REDD (dans un scénario BAU).

Inclusion des différents secteurs d'activités et catégories d'utilisateurs des forêts, types de forêts et catégories de mesures d'atténuation.

Capacité de s'adapter afin de répondre aussi bien à diverses conditions locales qu'à de futurs changements imprévus à toutes échelles géographiques. D'éventuels compromis entre flexibilité et robustesse doivent être envisagés. 


\begin{tabular}{ll}
\hline Critère de l'efficacité \\
\hline $\begin{array}{l}\text { Caractère } \\
\text { vérifiable des } \\
\text { mesures }\end{array}$ & $\begin{array}{l}\text { Pour être vérifiables, les mesures doivent (i) être fondées sur } \\
\text { une technologie permettant de prendre des mesures exactes et } \\
\text { complètes ; et (ii) avoir la capacité de réaliser ces mesures. }\end{array}$ \\
$\begin{array}{l}\text { Déplacement } \\
\text { des émissions } \\
\text { (fuites) }\end{array}$ & $\begin{array}{l}\text { Des fuites peuvent se produire à l'intérieur d'un même pays } \\
\text { et d'un pays à l'autre, ainsi que dans le contexte d'activités } \\
\text { d'utilisation des terres (par ex. passage de la déforestation à } \\
\text { la dégradation au cas où seule l'une de ces deux activités est } \\
\text { concernée). En général, plus l'échelle et le champ d'application de }\end{array}$ \\
$\begin{array}{ll}\text { la REDD seront étendus, plus le risque de fuite sera faible. } \\
\text { Permanence et } \\
\text { responsabilité }\end{array}$ & $\begin{array}{l}\text { On entend par permanence le processus permettant de garantir } \\
\text { les réductions à long terme, c.à- d. d'éviter les réductions } \\
\text { d'émissions qui ne retardent (les effets de ces émissions) } \\
\text { que pendant une brève période. Des mesures engageant les } \\
\text { responsabilités peuvent s'appliquer au cas où le principe de la } \\
\text { permanence n'aurait pas été respecté. }\end{array}$ \\
\hline $\begin{array}{l}\text { Effet sur d'autres } \\
\text { mesures } \\
\text { d'atténuation }\end{array}$ & $\begin{array}{l}\text { Un risque réel tient à ce que les actions engagées au titre de } \\
\text { la REDD le soient au détriment d'autres mesures d'atténuation } \\
\text { des effets du changement climatique. Il est difficile de mesurer } \\
\text { l'impact de ces effets. }\end{array}$ \\
\hline
\end{tabular}

\subsubsection{Efficience}

Par efficience on entend l'obtention de résultats à moindres coûts pour une action engagée au titre de la réduction des émissions. Divers coûts doivent être pris en compte dans toute élaboration de modèle pour la REDD. Ces coûts peuvent être ventilés en frais d'établissement (investissements de renforcement initial des capacités, voir : Eliasch 2008) et frais de fonctionnement liés à la réduction des émissions. Cette dernière catégorie peut à son tour être divisée en frais d'exploitation (ou coûts de protection de la forêt, voir : Eliasch 2008) et coûts d'opportunité (voir chapitre 5). Outre les coûts d'opportunité, les coûts de transaction doivent être pris en compte dans le calcul des dépenses encourues par les propriétaires fonciers au titre de leur participation au mécanisme REDD ; c'est un aspect parfois négligé aux retentissements insondables. Le Tableau 2.3 résume les éléments liés au critère de l'efficience. 
Tableau 2.3. Critère de l'efficience

\begin{tabular}{ll}
\hline Critère de l'efficience & lnvestissements initiaux \\
$\begin{array}{l}\text { (capitaux de départ pour } \\
\text { renforcer les capacités) }\end{array}$ & $\begin{array}{l}\text { Coûts liés à la mise en place du mécanisme REDD, } \\
\text { notamment de l'infrastructure technique et des } \\
\text { structures de gouvernance et, surtout, des actions de } \\
\text { formation et de renforcement des capacités nécessaires. }\end{array}$ \\
$\begin{array}{l}\text { Frais d'exploitation } \\
\text { (coûts liés à la protection } \\
\text { de la forêt) }\end{array}$ & $\begin{array}{l}\text { Frais d'exploitation d'un mécanisme REDD qui, outre un } \\
\text { examen régulier de suivi, inclue une série de politiques et } \\
\text { de mesures comme la mise en application des législations } \\
\text { forestières et les réformes foncières. }\end{array}$ \\
$\begin{array}{l}\text { Coûts d'opportunité des } \\
\text { propriétaires fonciers }\end{array}$ & $\begin{array}{l}\text { Les coûts d'opportunité correspondent au manque à } \\
\text { gagner résultant de l'application de meilleurs choix } \\
\text { (non forestiers) d'utilisation des terres, par ex., la somme } \\
\text { minimale à verser à un propriétaire pour l'inciter à cesser } \\
\text { toute activité de déboisement et de dégradation dans la } \\
\text { forêt (paiement de compensation). Ce facteur coût fera } \\
\text { partie des éléments-clé du système national des PSE. }\end{array}$ \\
\hline $\begin{array}{l}\text { Coûts de transaction des } \\
\text { propriétaires fonciers }\end{array}$ & $\begin{array}{l}\text { Pour participer au programme REDD, le propriétaire } \\
\text { foncier encourra vraisemblablement des frais } \\
\text { additionnels (par ex. édifier des clôtures, obtenir des } \\
\text { certifications), qui devront être pris en compte dans les } \\
\text { paiements de compensation. }\end{array}$ \\
\hline
\end{tabular}

\subsubsection{Equité et bénéfices y associés}

La plupart des propositions présentées au titre de la REDD comportent des objectifs ne visant pas le climat mais la répartition des bénéfices et des coûts, les moyens de subsistance, la réduction de la pauvreté, la protection des droits, et/ou la biodiversité (chapitre 11). Les considérations liées à l'équité ont plusieurs dimensions, notamment la répartition équitable des bénéfices entre les pays et à l'intérieur même des pays ou encore les effets des activités REDD sur les communautés locales et autochtones. Les critères d'évaluation des bénéfices associés à l'équité comprennent le développement économique et la réduction de la pauvreté, la biodiversité, les droits et la gouvernance des forêts (Tableau 2.4). 
Chapitre 2 Quels sont les enjeux clé du modèle REDD et les critères d'évaluation des options ?

Tableau 2.4. Critère de l'équité et des bénéfices y associés

\begin{tabular}{|ll}
\hline Critère de l'équité & \\
\hline $\begin{array}{l}\text { Répartition équitable } \\
\text { entre les pays }\end{array}$ & $\begin{array}{l}\text { Un premier aspect d'un traitement équitable est lié à } \\
\text { la pauvreté, c.à-d. (i) à la capacité des pays pauvres à } \\
\text { participer à un projet REDD (par ex. surveillance, suivi et } \\
\text { contrôle - MRV - et conditions en matière de gouvernance); } \\
\text { et (ii) au traitement préférentiel à accorder aux pays les plus } \\
\text { pauvres (par ex. en fixant les niveaux de référence). } \\
\text { Un autre aspect d'un traitement équitable consiste à « ne } \\
\text { pas pénaliser les actions anticipées »ni «à récompenser les } \\
\text { mauvaises politiques »; si les niveaux de référence étaient } \\
\text { basés sur une déforestation antérieure, il conviendrait de ne } \\
\text { pas pénaliser un « retard de développement ». }\end{array}$ \\
$\begin{array}{l}\text { Répartition équitable } \\
\text { à l'intérieur des pays }\end{array}$ & $\begin{array}{l}\text { On se réfère ici à l'équité dans le pays même, c.-à-d. à la } \\
\text { répartition des coûts et des bénéfices entre tous les niveaux } \\
\text { de l'administration (gouvernements locaux par rapport } \\
\text { au gouvernement central) et entre tous les partenaires } \\
\text { intervenant dans l'utilisation des terres. }\end{array}$ \\
\hline $\begin{array}{l}\text { Effets sur les } \\
\text { communautés locales } \\
\text { et autochtones }\end{array}$ & $\begin{array}{l}\text { Le Plan d'Action de Bali reconnaitt le rôle joué par les } \\
\text { communautés locales et autochtones dans les activités liées } \\
\text { à la REDD. Les conséquences sur le plan pratique en sont } \\
\text { la reconnaissance des droits traditionnels et l'inclusion des } \\
\text { communautés autochtones dans le processus de décision } \\
\text { de la REDD. }\end{array}$ \\
\hline
\end{tabular}

\section{Critère des bénéfices associés}

Développement économique et réduction de la pauvreté Biodiversité
La REDD peut soit faciliter soit gêner le développement économique aux niveaux national et sous-national et toucher aussi les secteurs d'activités économiquement dépendants des forêts ainsi que les économies nationales dans leur ensemble.

Les objectifs en matière de carbone et de biodiversité sont essentiellement compatibles mais des compromis pourraient être trouvés, par exemple, dans la répartition géographique des fonds (il pourrait y avoir des chevauchements entre des zones critiques pour la biodiversité d'une part, et le carbone, d'autre part).

Situation en matière de droits et de gouvernance des forêts
La REDD a le potentiel nécessaire pour améliorer la situation des droits et la gouvernance forestière, par ex. à travers des systèmes d'information plus transparents sur les forêts. Elle constitue aussi un risque dès lors que les sommes potentiellement considérables qu'elle est en mesure de générer donnent lieu à des pratiques de corruption, de mauvaise gestion et de " capture des élites ». 


\subsection{Conclusions}

D'importantes décisions devront être prises quant au modèle à appliquer pour faire progresser la REDD et mettre au point son architecture globale. La CCNUCC doit fixer de toute urgence des lignes directrices pour certains des enjeux les plus importants. Il conviendra de procéder à une évaluation minutieuse des avantages et inconvénients des différentes options ainsi que de leurs implications pour être à même de faire un choix judicieux et bien informé. Pour devenir un mécanisme d'atténuation internationalement accepté, la REDD devra essentiellement se conformer au moins aux trois critères suivants : (i) l'efficacité, à travers des actions aboutissant à de réelles réductions des émissions, (ii) l'efficience, en obtenant ces réductions à moindre coût, et (iii) l'équité, à travers des bénéfices associés et en limitant les concessions mutuelles non souhaitables sur le plan social et écologique. 


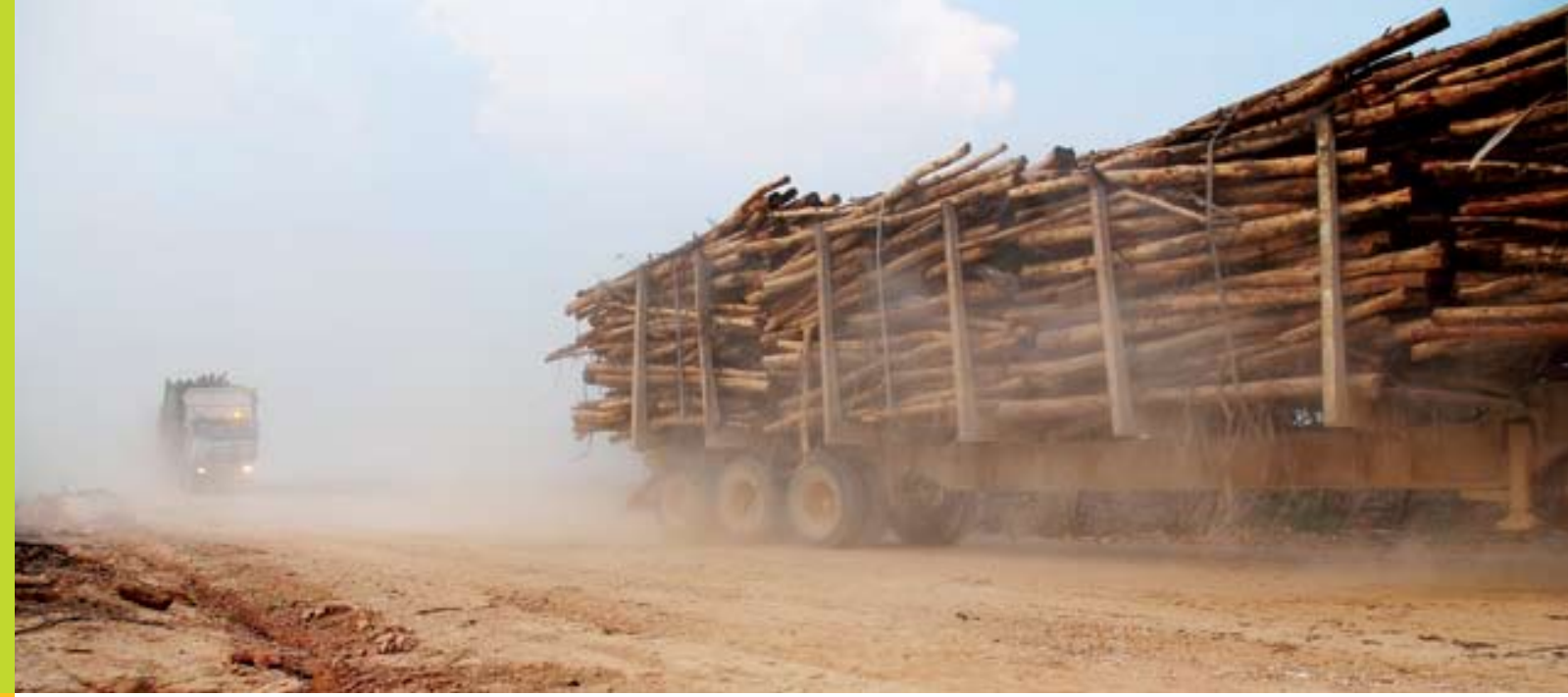

\section{Chapitre 3}

\section{Quels coûts et quel potentiel pour la REDD?}

Ruben N. Lubowski

\subsection{Introduction}

Il est scientifiquement reconnu que toute riposte pour éviter de dangereuses perturbations du système climatique - par exemple, une hausse des températures supérieure à 2 degré centigrades d'ici la fin du siècle - passe nécessairement par la réduction rapide et à grande échelle des émissions des gaz à effet de serre (GES) causées par les pays développés et les principaux pays en développement émetteurs de GES. La réduction des émissions provenant des forêts tropicales ombrophiles offre une possibilité immédiate d'atténuer une source substantielle d'émissions à des coûts pouvant être estimés à un niveau relativement faible. Les actions engagées pour réduire les émissions liées à la déforestation et à la dégradation des forêts (REDD) pourraient aussi constituer une "stratégie passerelle » intéressante pour réduire les émissions à brève échéance tout en gagnant du temps pour s'adapter à un avenir où de faibles émissions de $\mathrm{CO}_{2}$ est une réalité.

Dans ce chapitre nous examinons certains des enjeux importants qui détermineront la politique et l'architecture de la REDD : Combien coûtera la REDD ? Quelle sera l'influence globale de la REDD sur la stratégie globale visant à réduire les émissions de GES ? Quels en seront les effets sur le prix du 
carbone et les actions visant à réduire les émissions dans d'autres secteurs ? Nous mettons l'accent sur la façon dont différents modèles économiques apportent des réponses à ces questions.

\subsection{Combien coûtera la REDD ?}

\subsubsection{Types de coûts liés à la REDD}

Les coûts évalués pour réduire les émissions liées à la déforestation et à la dégradation des forêts (REDD) varient en fonction des données, de l'approche de modélisation utilisée et des types de coûts qui entrent en compte. Dans les études qui ont été faites, les coûts sont rapportés à l'offre ou à la demande de réductions d'émissions REDD, ou aux deux. La plupart des estimations portent sur les " coûts d'opportunité " liés à une déforestation évitée au sens où l'entend un propriétaire foncier (c.-à-d. le renoncement à des bénéfices économiques qui pourraient être tirés d'une autre utilisation des terres), sans les coûts associés à la mise en place des capacités institutionnelles et au fonctionnement du mécanisme REDD.

Certains modèles économiques présentent des « courbes d'offre » estimées (" courbes de coûts marginaux ») pour tout un éventail de coûts correspondant à des réductions progressives d'émissions résultant des forêts (Figure 3.1). La pente de la courbe ascendante des coûts montre qu'à de faibles taux de réduction d'émissions, il est possible de maintenir les coûts à des niveaux relativement bas en protégeant, par exemple, uniquement les terres les moins onéreuses. On constate que l'écart se creuse avec le différentiel des coûts à mesure que les taux de réduction d'émissions augmentent, les coûts " marginaux " augmentant aussi puisqu'il faudra prendre des mesures de protection supplémentaires qui s'étendront nécessairement aux terres les plus onéreuses. Par exemple, les estimations concernant les coûts globaux d'opportunité font plus que doubler quand le taux de protection de la forêt amazonienne du Brésil passe de 94 à 100 pour cent en raison du potentiel agricole considérable de terres ne représentant pourtant que 6 pour cent de la superficie totale (Nepstad et al. 2007).

Au nombre des coûts de mise en œuvre des politiques REDD figurent les frais initiaux de lancement des activités de "renforcement des capacités ", les " frais d'administration " liés à la surveillance, à la mise en application et aux autres activités nécessaires pour mener à bien un programme REDD et les "frais de transaction " associés à une mise en contact des acheteurs et des vendeurs dans de bonnes conditions. Les pays n'ont pas les mêmes capacités de réduire les émissions provenant des forêts tropicales et les coûts de mise en œuvre varieront d'un pays à l'autre en fonction des capacités et stratégies nationales. Les besoins ponctuels en matière de renforcement des capacités et de réforme des politiques au titre de la REDD dans 40 pays ont été récemment estimés à un total de 4 milliards de dollars US (Eliasch 2008). En outre, les 
coûts afférents à la création et à la validation de crédits REDD dépendront essentiellement des règles fixant les niveaux de référence qui détermineront les conditions dans lesquelles seront effectuées les compensations au titre de la REDD (voir chapitre 6).

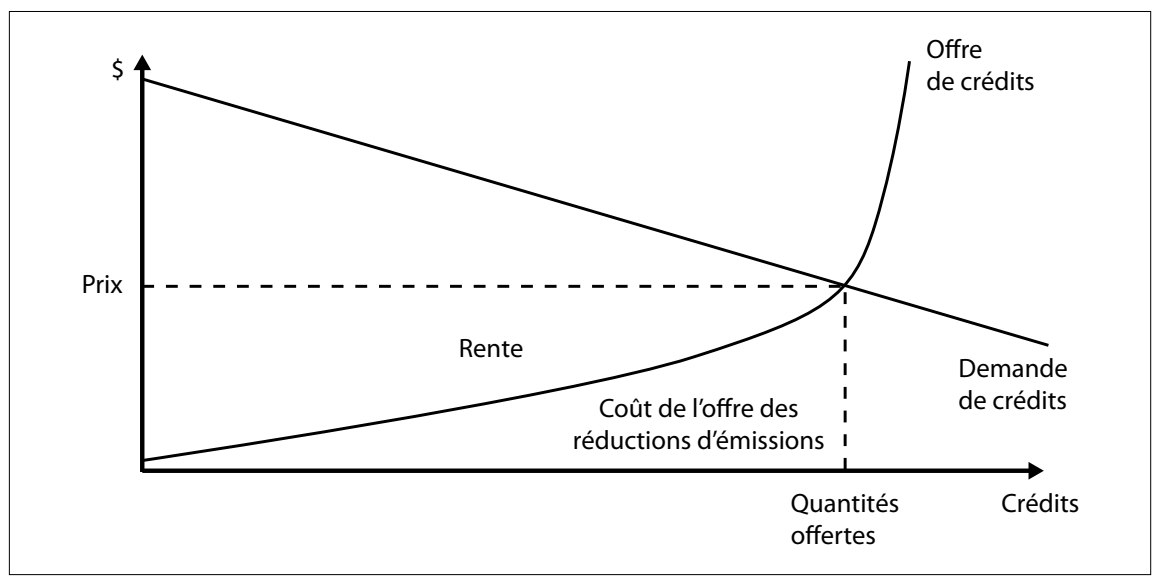

Figure 3.1. Offre et demande de 'crédits' REDD

\subsubsection{Approches de modélisation}

La plupart des estimations concernant les coûts associés à la REDD sont tirées d'études " ascendantes » ou « techniques » basées sur des informations détaillées relatives à des activités particulières conduites sur des sites particuliers, à des prix déterminés. Les modèles "descendants " en revanche sont plus globaux et prennent en compte les interactions du marché des matières premières aussi bien la demande que l'offre. Les estimations indiquées par ces derniers pour les coûts de projets REDD à grande échelle sont en général plus élevées, en partie parce qu'elles tiennent compte des réactions du marché (voir Tableau 3.1) à mesure que les actions visant à réduire la déforestation feront baisser l'exploitation du secteur forestier et les superficies boisées converties en terres agricoles. Il en résultera une moindre croissance de l'offre de soja, bétail et bois qui entraînera une augmentation du prix de ces produits, incitant ainsi à une reprise des activités de déforestation tant que la demande non satisfaite ne sera pas complètement absorbée. Ces réactions du marché rendront la REDD plus coûteuse et accroîtront le risque de "fuites " en fournissant les motivations nécessaires à un déplacement des activités de déforestation dans une autre zone. 
Tableau 3.1. Réduire de moitié la déforestation dans le monde : comparaison entre les modèles ascendants et descendants

\begin{tabular}{|c|c|c|}
\hline & $\begin{array}{l}\text { Modèle ascendant } \\
\text { Analyse dans huit pays tropicaux } \\
\text { (Grieg-Gran dans Eliasch 2008) }\end{array}$ & $\begin{array}{l}\text { Modèle descendant } \\
\text { Examen de trois modèles } \\
\text { globaux d'utilisation des terres } \\
\text { (Kindermann et al. 2008) }\end{array}$ \\
\hline $\begin{array}{l}\text { Coût d'une } \\
\text { déforestation } \\
\text { réduite de } \\
\text { moitié }\end{array}$ & 7 milliards de dollars US/an & $\begin{array}{l}\text { 17,2 à } 28 \text { milliards de dollars } \\
\text { US/an }\end{array}$ \\
\hline Echéance & $\begin{array}{l}\text { Immédiate ; et réductions } \\
\text { annuelles assurées sur une } \\
\text { période de } 30 \text { ans }\end{array}$ & En 2050 \\
\hline Frais encourus & $\begin{array}{l}\text { Coûts d'opportunité liés à la } \\
\text { protection des forêts (par ex. } \\
\text { coûts liés à l'offre de réduction } \\
\text { d'émissions à la Figure } 3.1 \text { ) ; } \\
\text { frais administratifs estimés } \\
\text { entre } 233 \text { et } 500 \text { millions dollars } \\
\text { US/an pour la REDD ; et coûts } \\
\text { ponctuels estimés à } 50 \text { millions } \\
\text { de dollars US pour dresser les } \\
\text { inventaires forestiers nationaux } \\
\text { dans } 25 \text { pays, auxquels il faut } \\
\text { ajouter de } 7 \text { à } 17 \text { millions dollars } \\
\text { US/an pour les administrer }\end{array}$ & $\begin{array}{l}\text { Les courbes des coûts } \\
\text { d'opportunité sont des courbes } \\
\text { estimées. Les coûts totaux } \\
\text { tiennent compte des coûts } \\
\text { d'opportunité liés à l'offre de } \\
\text { réduction d'émissions, auxquels } \\
\text { s'ajoutent les « rentes » (profits) } \\
\text { versées aux fournisseurs REDD } \\
\text { vendant leurs réductions au prix } \\
\text { unique du marché (Figure 3.1). } \\
\text { Cela correspond à une dépense } \\
\text { pour un acheteur sur un } \\
\text { marché de concurrence ; les } \\
\text { « rentes » que perçoit le vendeur } \\
\text { constituent une redistribution } \\
\text { des ressources et non un coût } \\
\text { pour la société. Toutefois, } \\
\text { ces rentes ont un effet sur le } \\
\text { coût réel, autrement dit sur } \\
\text { la capacité d'un programme } \\
\text { REDD à optimiser ses réductions } \\
\text { d'émission avec un budget limité. }\end{array}$ \\
\hline Commentaires & $\begin{array}{l}\text { Les prix sont fixés pour les } \\
\text { matières premières }\end{array}$ & $\begin{array}{l}\text { Les effets du marché sont pris en } \\
\text { compte (par ex. hausses de prix } \\
\text { quand l'offre baisse), ce qui tend } \\
\text { à relever les coûts }\end{array}$ \\
\hline
\end{tabular}

Les différences observées dans le scénario modélisé « de référence » indiquant ce que serait une déforestation sans mise en application de stratégies REDD ont aussi un effet sur les coûts estimés pour la REDD. Une déforestation plus forte dans un scénario de la poursuite de la tendance actuelle (BAU) entraînerait un plus grand nombre d'émissions potentielles à réduire mais pourrait aussi, dans cette simulation, exercer une pression plus forte sur les forêts et, par conséquent, 
contribuer à relever les coûts liés à la protection de la forêt. D'autres différences constatées en matière de données et d'hypothèses contribuent aussi à des estimations diverses des coûts liés à la REDD (Tableau 3.2).

Tableau 3.2. Effets de l'inclusion de différentes caractéristiques des modèles sur les coûts estimés de la REDD

\section{Choisir les caractéristiques à inclure dans le modèle}

\section{Effet sur les coûts}

Effets sur les prix : la baisse de l'offre de bois, cultures, etc. fait monter les prix et, par conséquent les coûts d'opportunité liés à la protection de la forêt.

Nombre de déterminants de la déforestation modélisés : la prise en compte de facteurs supplémentaires comme l'exploitation du bois et l'agriculture, accroîtront les coûts d'opportunité liés à la protection des forêts ; à l'avenir, la prise en compte de nouveaux facteurs comme les biocarburants - plutôt que d'extrapoler à partir de facteurs de déforestation antérieurs - peut aussi accroître les coûts estimés.

Mise en œuvre et coûts de transaction, risques liés aux investissements.

Bénéfices tirés de la conversion en terres agricoles par rapport aux coûts y afférents : le profit ponctuel réalisé (bois récolté après défrichement) augmentera le coût des actions liées à la protection de la forêt.

Une « élasticité de la transformation » (paramètre fictif pour décrire la convertibilité des terres forestières à d'autres utilisations) plus grande, correspondra une augmentation des coûts dans certains modèles.

Densité de carbone /libération de $\mathrm{CO}_{2}$ : plus le taux d'émissions évitées par ha protégé sera important, plus le coût par tonne de carbone sera réduit.

Bénéfices liés au bois dans les forêts protégées (par ex. gestion durable des forêts).

Champ d'application du modèle REDD (activités forestières, secteurs, pays, gaz) : plus il sera vaste, moins il y aura de fuites et plus il y aura de possibilités de réaliser des réductions globales à faible coût.

\section{Champ d'application des incitations : plus la couverture sera} complète, moins il y aura de fuites et plus les coûts seront bas.

Cibler les incitations : fonder les paiements sur les réductions d'émissions diminue les transferts aux non-émetteurs et donc les coûts (pour les acheteurs), mais des considérations comme celles visant à éviter les « fuites » et à assurer l'équité doivent aussi être prises en compte. 


\subsection{Quels seront les effets de la REDD sur la stratégie globale de réduction des émissions?}

La prise en compte de la déforestation et d'autres options d'utilisation des terres pour réduire les émissions dans le contexte des modèles simulés pour le climat est chose relativement nouvelle. Néanmoins, les résultats du Forum 21 sur la modélisation de l'énergie [Energy Modeling Forum 21 (Rose et al. 2007)] et d'autres études montrent qu'une diminution de la déforestation - grâce à la plantation d'arbres (boisement et reboisement, B/R), aux changements apportés en matière de gestion forestière, et à d'autres actions engagées dans les forêts pour atténuer les émissions de GES - pourrait entraîner d'importantes économies sur le prix à payer pour atteindre les objectifs de stabilisation du climat d'ici au siècle suivant (Tableau 3.3, Fischer et al. 2007).

Ces économies pourraient permettre de plus grandes réductions d'émissions - au même coût global - que celles susceptibles de se produire en l'absence d'un mécanisme REDD. Les montants de ces économies qui résultent de l'application de mesures d'atténuation du changement climatique dans les forêts, sont estimés à 2 trillions de dollars US et pourraient financer des scénarios de réduction plus contraignants permettant, selon les modèles de simulation, de diminuer encore de 10 pour cent les émissions, ce qui correspondrait à $0,25^{\circ} \mathrm{C}$ de réchauffement en moins, à atteindre au cours de ce siècle (voir Tableau 3.3). Le gain potentiel - à travers des activités REDD - dépend des taux de concentration des GES-cibles dans l'atmosphère et des différentes options disponibles pour réduire les émissions. Plus le nombre d'options possibles est élevé, plus le sera aussi celui des sources potentielles de réductions à faible coût sans oublier un effet corollaire qui est de faire diminuer la dépendance vis-à vis d'une option qui serait la seule à permettre d'atteindre un objectif de réduction d'émissions à moindres frais. Une autre hypothèse critique pouvant avoir un effet sur le rôle estimé de la REDD dans les modèles de simulation climatiques est celle du développement attendu des futures technologies basées sur les biocarburants (Tableau 3.3). Combinée au piégeage et à la séquestration du carbone, la production de biomasse dans le but de générer de l'électricité, en particulier, pourrait théoriquement devenir un puissant concurrent pour accaparer des terres si ce moyen permet de fabriquer de l'énergie sans émissions négatives de $\mathrm{CO}_{2}$ (cf. Obersteiner et al. 2001). 
Tableau 3.3. Contribution potentielle de la REDD à une réduction des coûts et à l'achat d'un supplément de réductions d'émissions : comparaison entre plusieurs modèles

\begin{tabular}{|c|c|}
\hline Modèle et type & Résultats \\
\hline $\begin{array}{l}\text { WITCH associé } \\
\text { au GTM (analyse } \\
\text { d'évaluation } \\
\text { intégrée ; } \\
\text { Tavoni et al. 2007) }\end{array}$ & $\begin{array}{l}\text { L'inclusion de mesures de réduction des émissions dues à la } \\
\text { déforestation, comme des activités B/R et des changements } \\
\text { de gestion forestière, permettrait d'atteindre un objectif de } \\
550 \text { e- } \mathrm{CO}_{2} \text { parties par million par volume (ppmv) atmosphère } \\
\text { au même coût global que pour un objectif de } 600 \text { ppmv } \\
\text { n'incluant pas de telles mesures d'atténuation. Ces dernières } \\
\text { permettent de réaliser une économie globale d'environ } 2 \\
\text { trillions de dollars US; il en résulterait une élévation de la } \\
\text { température estimée à } 0,25^{\circ} \mathrm{C} \text { de moins d'ici la fin du siècle } \\
\text { sans entraîner de frais supplémentaires (comparés à ceux } \\
\text { encourus au titre des réductions dans le secteur de l'énergie). }\end{array}$ \\
\hline $\begin{array}{l}\text { GLOCAF associé } \\
\text { au GCOMAP et au } \\
\text { modèle de groupe } \\
\text { IIASA (analyse } \\
\text { d'évaluation } \\
\text { intégrée; } \\
\text { Eliasch 2008) }\end{array}$ & $\begin{array}{l}\text { Les coûts afférents à une réduction globale de } 50 \% \text { - par } \\
\text { rapport aux niveaux de } 1990 \text { - des émissions d'ici } 2050 \\
\text { (stabilisées à } 475 \text { e- } \mathrm{CO}_{2} \text { ) pourraient être revus à la baisse ( } 25 \\
\text { à } 50 \% \text { en } 2030 \text { et } 20 \text { à } 40 \% \text { en 2050) en cas d'inclusion des } \\
\text { réductions liées à la déforestation et de prise en compte des } \\
\text { activités B/R. Les économies réalisées (approx. } 2 \text { trillions dollars } \\
\text { US) pourraient financer les dépenses afférentes à la réalisation } \\
\text { d'un objectif global de } 10 \% \text { d'émissions en moins. }\end{array}$ \\
\hline $\begin{array}{l}\text { MESSAGE (analyse } \\
\text { d'évaluation } \\
\text { intégrée ; cf. Rao et } \\
\text { Riahi } 2006 \text {; } \\
\text { Riahi et al. 2006) }\end{array}$ & $\begin{array}{l}\text { Inclut une vaste gamme d'options d'utilisation des terres : } \\
\text { déforestation évitée, B/R, atténuation des activités agricoles, } \\
\text { biocarburants (combustibles liquides et énergie générée à } \\
\text { travers le piégeage et la séquestration du carbone). Les options } \\
\text { impliquant des biocarburants sont en forte concurrence avec } \\
\text { les forêts ; les options foresterie et biocarburants devraient } \\
\text { contribuer respectivement à hauteur de } 1 \text { à } 2 \% \text { et } 6 \text { à } 24 \% \\
\text { pendant les } 50 \text { prochaines années, et dans une proportion de } \\
4 \text { à } 8 \% \text { et } 14 \text { à } 29 \% \text { d'ici au siècle prochain pour atteindre une } \\
\text { stabilisation d'environ } 650 \text { e- } \mathrm{CO}_{2} \text { ppmv. Le modèle prévoit une } \\
\text { conversion de périmètres substantiels de forêts primaires en } \\
\text { forêts plantées d'exploitation. }\end{array}$ \\
\hline $\begin{array}{l}\text { GRAPE (analyse } \\
\text { d'évaluation } \\
\text { intégrée ; } \\
\text { Kurosawa 2006) }\end{array}$ & $\begin{array}{l}\text { Inclut la déforestation évitée, le boisement/reboisement } \\
\text { (B/R), I'atténuation des activités agricoles, et les biocarburants } \\
\text { sous forme de combustibles liquides (mais pas à des fins de } \\
\text { production d'énergie à travers le piégeage et la séquestration } \\
\text { du carbone). Les activités de foresterie jouent ici un grand } \\
\text { rôle et représenteraient } 55 \% \text { et } 15 \% \text { des réductions } \\
\text { respectivement prévues d'ici les } 50 \text { à } 100 \text { prochaines années. }\end{array}$ \\
\hline $\begin{array}{l}\text { GTEM (modèle } \\
\text { « d'équilibre } \\
\text { général »; Jakeman } \\
\text { et Fisher 2006) }\end{array}$ & $\begin{array}{l}\text { Inclut la déforestation évitée, le boisement/reboisement } \\
\text { (B/R) et l'atténuation des activités agricoles; exclut les } \\
\text { biocarburants. Dans I'hypothèse d'un objectif de } \\
\text { concentration de } 650 \text { e- } \mathrm{CO}_{2} \text { l la contribution de la } \\
\text { foresterie est estimée à } 11 \% \text { de la réduction totale dans les } \\
50 \text { prochaines années et, toutes options d'atténuation basées } \\
\text { sur l'utilisation des terres confondues, ce scénario devrait } \\
\text { permettre d'économiser de } 1.6 \text { à } 7.6 \text { trillions de dollars US } \\
\text { selon que sont ou non incluses des options d'atténuation } \\
\text { autres que celles visant à réduire les émissions de } \mathrm{CO}_{2} \text {. }\end{array}$ \\
\hline
\end{tabular}


La plupart des études axées sur la REDD mettent l'accent sur son potentiel économique en supposant que les cadres institutionnels et les capacités nécessaires existent déjà et peuvent être facilement mobilisés pour mettre immédiatement en œuvre le mécanisme REDD dans le monde entier. Ce ne sont cependant pas tous les pays qui choisiront d'adhérer à un accord international sur le climat ou qui seront en mesure de réduire leurs émissions liées à la déforestation à brève échéance. Ces obstacles institutionnels et politiques font baisser l'échelle réaliste des réductions possibles et leur impact global réel. Des incitations contradictoires pour promouvoir la réduction globale à travers le monde des émissions de GES et de celles liées à la déforestation et à la dégradation des forêts (REDD) pourraient créer un terrain propice aux «fuites » ou au « déplacement » d'émissions d'un pays à l'autre, les réductions d'émissions pour un pays étant potentiellement neutralisées par des augmentations ailleurs. Ainsi, Gan et McCarl (2007), par exemple, ont estimé les fuites dans le secteur des produits forestiers sur le plan international à un niveau très élevé atteignant entre 42 et 95 pour cent.

\subsection{Quel sera l'effet de la REDD sur le prix du carbone et quelles seront les actions à mener pour réduire les émissions dans d'autres secteurs?}

Les avantages potentiels présentés par la REDD du point de vue des coûts y afférents pourraient détourner l'intérêt de la nécessité de réduire les émissions dans d'autres secteurs au cas où les crédits REDD deviendraient interchangeables sans restrictions avec d'autres crédits GES. L'un des risques perçus est que la REDD pourrait "inonder " le marché du carbone et faire perdre de vue le signal prix qui est de mettre en place et de déployer des technologies basées sur une énergie propre. L'impact de la REDD sur les prix du carbone et les incitations technologiques dépend de plusieurs facteurs, notamment :

- des quantités d'émissions pouvant effectivement résulter d'une déforestation évitée et des crédits pouvant être attribués à ce titre dans le cadre de la REDD, étant entendu que les chiffres des émissions comme des crédits dépendent du coût total de la REDD, des pays qui participent au mécanisme et des conditions d'attribution des crédits (ou droits d'émission).

- de la demande en matière de REDD, basée sur l'objectif global de réduction des émissions ainsi que sur le coût et la disponibilité d'autres options d'atténuation des émissions. Au cas où des objectifs plus contraignants seraient fixés, la demande de REDD augmentera et la réduction deviendra plus onéreuse pour d'autres secteurs. 
- des options pour appliquer des actions de «comptabilisation " anticipées pour réduire les émissions en prévision d'obligations futures, relevant ainsi potentiellement la demande actuelle de REDD.

- des règles sur la "fongibilité " des crédits REDD. Restreindre l'utilisation de la REDD et des autres options d'atténuation reviendrait à relever le prix du carbone (et du coût total).

Tavoni et al. (2007) estiment que la mise en ouvre globale de la REDD ainsi que des activités de boisement/reboisement (B/R) et des changements de modes de gestion forestière retarderaient le déploiement de certaines technologies et réduiraient les investissements en recherche et développement dans le secteur de l'énergie d'environ 10 pour cent pour un objectif de réduction d'émissions donné fixe. Anger et Sathaye (2006) concluent à une réduction de 40 pour cent du prix du carbone si la REDD est introduite dans un marché qui autorise l'attribution de crédits sans plafonnement en récompense de la prise de mesures d'atténuation dans un pays en développement à travers le MDP. D'autres études rapportent des effets plus insidieux qui varient en fonction de la scène politique.

D'après Eliasch (2008), l'introduction de crédits REDD associés à de modestes limitations quantitatives des réductions n'aurait qu'un impact négligeable sur le prix du carbone dans l'Union Européenne, même si les pays ont la capacité suffisante pour honorer de 50 à 85 pour cent - selon le degré de rigidité de l'objectif de l'UE - de la part (quota) de réductions qui leur a été attribuée à travers des crédits internationaux. L'impact proportionnel de la REDD sur le prix ne pourra être évalué avec précision qu'en tenant compte des facteurs qui ont déterminé la forme des courbes des coûts, y compris des coûts correspondant à d'autres options potentielles.

Des objectifs de longue durée, suffisamment ambitieux et crédibles, devraient inciter à une participation anticipée au marché du carbone et à économiser les crédits qui pourront être utilisés dans un contexte de resserrement des objectifs plus tard. Au vu de toutes ces options de " comptabilisation " Piris-Cabezas et Keohane (2008) estiment qu'un programme REDD global abaisserait le prix du carbone de 14 pour cent sur le marché mondial et qu'en ajoutant toutes les mesures d'atténuation existantes dans le secteur forestier cette baisse atteindrait 31 pour cent pour un objectif de réduction d'émissions fixe donné. Le fait de doubler l'offre estimée de crédits REDD n'aurait qu'un effet relativement peu important sur le prix modélisé étant donné que les crédits additionnels sont " comptabilisés» et utilisés progressivement. Si la REDD permet ainsi de constituer un stock de réductions d'émissions à un coût relativement faible, ce " portefeuille " pourrait aussi réduire les effets de la volatilité des prix du fait qu'il représente une garantie contre toute future flambée imprévue des prix. 


\subsection{Conclusion}

Les éléments scientifiques les plus récents dont nous disposons laissent entendre que seule la mise en œuvre quasi-immédiate d'un programme global pour atteindre d'importantes réductions d'émissions de GES d'ici la fin de la première moitié du siècle en cours permettra de préserver les options visant à éviter de graves perturbations du système climatique mondial. Malgré diverses hypothèses, tout un éventail de modèles économiques indique que la REDD peut apporter une contribution substantielle pour stabiliser à des coûts efficients les taux de concentration des GES à l'échelle et au rythme qui s'imposent.

Les coûts et les échéances sont des éléments importants du programme REDD. Les économies de coûts estimées pour la REDD pourraient permettre d'atteindre des réductions d'émissions globales bien plus importantes et rapides que celles qui pourraient être atteintes en l'absence d'un programme REDD pour les mêmes quantités de dépenses globales engagées. La stabilisation des taux de concentration des GES à des niveaux sans risque oblige à d'ambitieux efforts pour réduire rapidement les émissions dues aussi bien aux forêts tropicales qu'à d'autres secteurs. La plupart des coûts stratégiques liés à la mise en œuvre de la REDD visent les " coûts d'opportunité » sans prendre en compte les coûts de transaction et les coûts liés au renforcement des capacités qui pourraient représenter des investissements supplémentaires conséquents. Cependant les économies de coûts réalisées sur le long terme à travers les mesures envisagées dans le secteur forestier par la plupart des modèles sont largement suffisantes pour couvrir ces éventuels surcoûts.

L'impact économique de la REDD dépendra des objectifs globaux du programme en matière de climat, de l'architecture, de l'élaboration et de la mise en ouvre de son plan stratégique, ainsi que de l'interchangeabilité des crédits REDD avec ceux du reste du marché des GES. Le risque potentiel « d'inondation » du marché du carbone par une offre REDD trop abondante peut être circonscrit par des stratégies allant de la fixation d'objectifs contraignants et de longue durée "comptabilisables ", à l'imposition de restrictions (plafonnements) modestes d'utilisation de la REDD et d'autres types de crédits.

Des réductions anticipées d'émissions peuvent être particulièrement précieuses au sens où elles constituent une assurance en garantissant le maintien d'options climatiques dans un contexte d'incertitudes scientifiques (Fisher et al. 2007). A mesure que les forêts tropicales disparaissent, la REDD devient aussi une option opportune et rentable - dont nous ne disposons que pour une période limitée pour réduire les émissions de $\mathrm{CO}_{2}$. La précarité et l'irréversibilité font partie de la nature même de la REDD - (une fois le processus de déforestation engagé, il ne pourra que se poursuivre) - et rendent encore plus urgente la nécessité de protéger les forêts tropicales dès maintenant plutôt que de se priver d'avoir recours à l'avenir à des options permettant de réduire les émissions globales. 


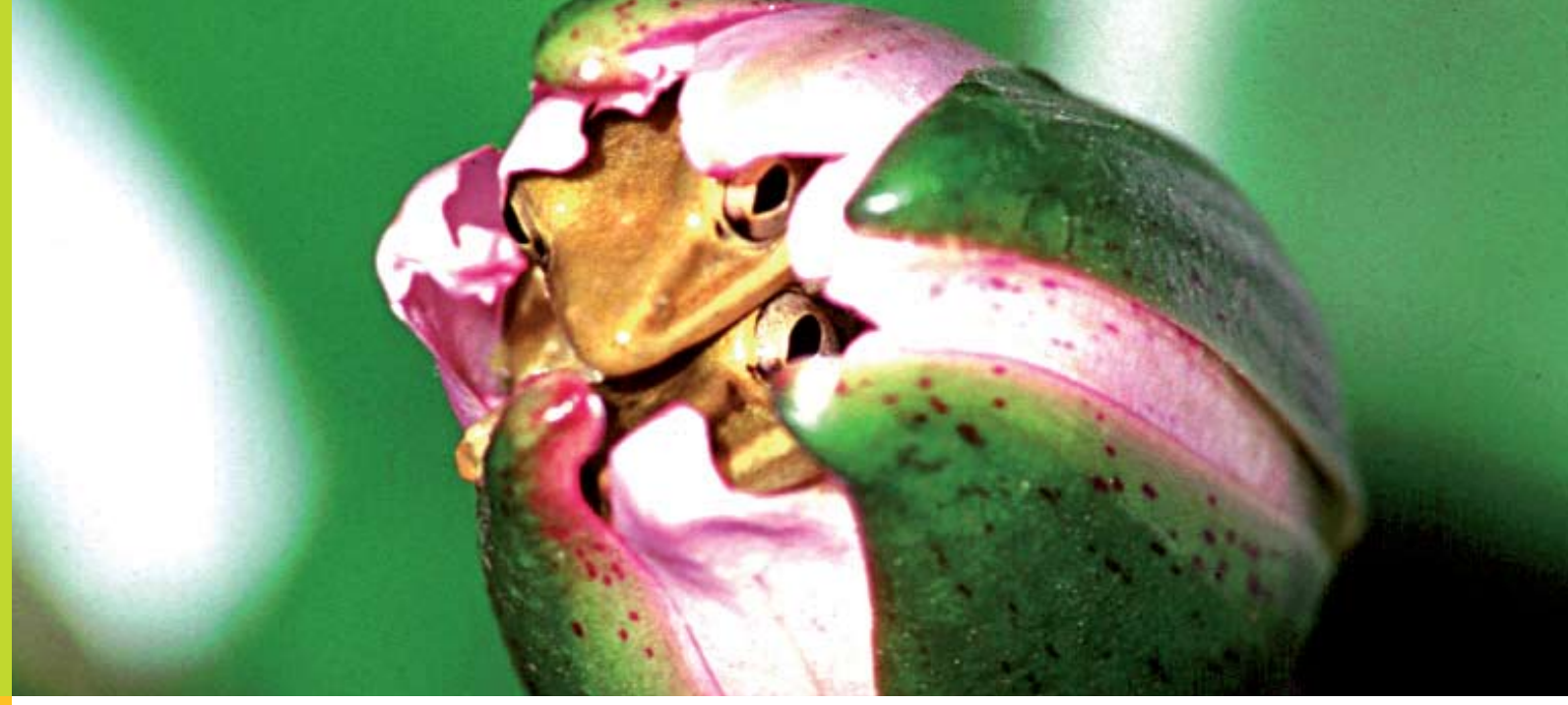

\title{
Chapitre 4
}

\section{Quelle échelle choisir pour la REDD?}

\author{
Arild Angelsen, Charlotte Streck, Leo Peskett, Jessica Brown et \\ Cecilia Luttrell
}

\subsection{Introduction}

Afin de promouvoir la réduction des émissions dues à la déforestation et à la dégradation des forêts, un mécanisme financier du même nom (REDD) a été proposé pour doter les pays en développement des incitations leur permettant de réduire les émissions liées au secteur forestier. Le mécanisme REDD pourrait faire partie de l'accord international sur le climat qui fait actuellement l'objet de négociations au sein de la Convention-cadre des Nations Unies sur les changements climatiques (CCNUCC). Une question clé du débat concerne le niveau géographique (échelle spatiale) d'offre et de comptabilisation des incitations destinées aux activités engagées au titre de la REDD. Cette comptabilisation devrait-elle s'effectuer : (i) au niveau sous-national (ou à celui du projet); (ii) au niveau national, ou (iii) aux deux niveaux (approche combinée) ? Ce chapitre décrit d'abord les trois approches envisagées pour la REDD et en évalue ensuite, pour chacune d'entre elles, les effets sur l'efficacité du carbone, l'efficience (rentabilité) des coûts et l'équité (les « $3 \mathrm{E} »)$.

Les différences existant entre les approches sous-nationales, nationales et combinées sont souvent floues. L'une des raisons est que l'expression 
" échelle spatiale » n'a pas le même sens dans les différentes propositions. Dans ce chapitre, on entend par échelle, le niveau de comptabilisation d'un mécanisme de financement international. Le niveau de comptabilisation sera généralement étroitement relié au niveau du compte à créditer (paiement), même si les accords conclus en matière de partage des bénéfices entre les niveaux sous-national et national peuvent estomper les contours des échelles. Le niveau de mise en œuvre est d'importance secondaire : au niveau national, la mise en œuvre peut inclure à la fois des projets qui sont mis en œuvre au niveau national et une stratégie nationale REDD qui attribue des crédits (droits d'émission) à des projets mis en œuvre par d'autres. De même, un projet mis en œuvre dans un contexte sous-national peut s'appuyer sur de bonnes politiques nationales qui facilitent la réalisation de ses objectifs.

\subsection{Trois échelles possibles pour la REDD}

\subsubsection{Approche sous-nationale}

La Figure 4.1 illustre les différences entre les trois options possibles. Dans l'approche sous-nationale, les activités REDD seront mises en œuvre dans un périmètre géographique bien défini ou en tant que projets par des individus, des communautés, des organisations non-gouvernementales (ONG), des sociétés privées ou des gouvernements nationaux ou locaux. Comme c'est le cas pour les trois approches, les activités créditrices effectuées au titre de la REDD devront s'appuyer sur des règles internationalement reconnues et acceptées en matière de surveillance, rapport de suivi et contrôle (Monitoring, Reporting and Verification, MRV), un système pour enregistrer les crédits attribués (les paiements effectués), et des arrangements institutionnels à la fois au niveau national (par ex. une autorité nationale désignée ou une autre ayant autorité pour approuver tous les projets) et au niveau international (par ex. un organe de supervision, un projet centralisé et un centre d'enregistrement des crédits). 


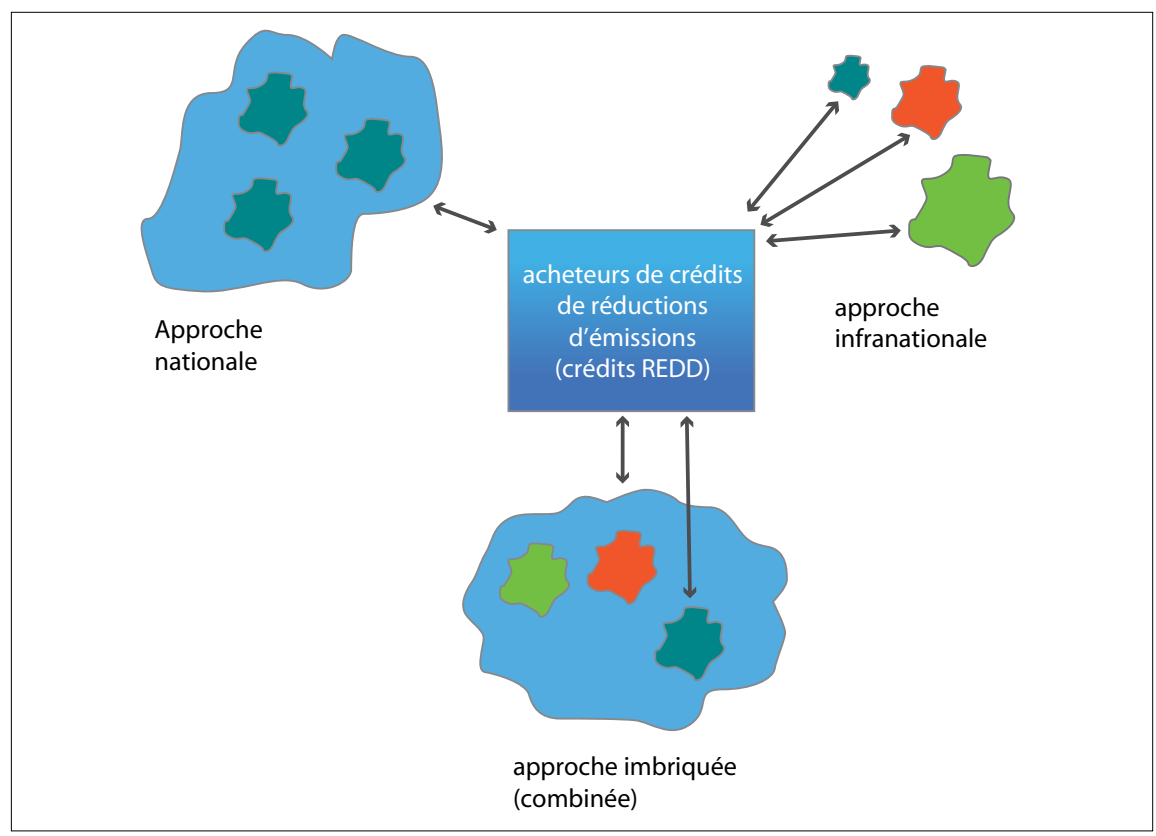

Figure 4.1. Les trois approches de la REDD en matière de comptabilisation et d'enregistrement des crédits d'émission Note : Les flèches indiquent la provenance de l'argent versé (acheteurs internationaux) et des informations reçues (entités (infra) nationales).

Les modalités et la procédure mises au point au titre du mécanisme pour un développement propre (MDP) du Protocole de Kyoto pourraient servir de modèle à l'élaboration de ce nouvel instrument institutionnel. Le MDP autorise les pays développés (visés à l'Annexe I) à compenser leurs émissions de gaz à effet de serre par un soutien apporté à des projets visant à réduire ces émissions dans des pays en développement. Dans le secteur forestier, seuls les projets de boisement/reboisement (B/R) sont actuellement éligibles et un seul projet a été approuvé à ce jour. Le MDP a mieux réussi dans d'autres secteurs, notamment celui de l'énergie ; sa valeur sur le marché des produits de base représentait 7,4 milliards de dollars US en 2007 (Hamilton et al. 2008). Les progrès des projets $\mathrm{B} / \mathrm{R}$ au titre du MDP sont lents en raison de règles et de méthodologies complexes et aussi parce que les frais d'enregistrement grèvent considérablement les coûts de transaction qui sont très élevés. D'autres obstacles sont liés au caractère non transférable des crédits temporaires attribués aux projets et à l'exclusion des crédits temporaires du système de quotas d'émissions (ETS) de l'UE. L'ETS est de loin le plus grand marché du carbone en place, avec un volume de transactions ayant atteint 50 milliards de dollars US en 2007, soit 58 pour cent du commerce mondial de carbone (Hamilton et al. 2008).

Un autre exemple d'une approche sous-nationale est celui du marché volontaire du carbone pour des projets visant à prévenir la déforestation. Les transactions 
sur ce marché ont atteint 330 millions de dollars US en 2007 (les projets liés aux forêts représentant 18 pour cent de la part du marché), représentant moins de 5 pour cent de la valeur du marché primaire du MDP. Les transactions sur le marché volontaire du carbone sont effectuées dans leur plus grande proportion (à hauteur de 80 pour cent) avec des acheteurs du secteur privé (Hamilton et al. 2008).

Comme le MDP réussit relativement bien dans d'autres secteurs et qu'il dispose d'une structure institutionnelle établie, certaines Parties à la CCNUCC, arguant en outre des difficultés que certains pays pourraient rencontrer à adopter l'approche nationale prévue au titre de la REDD, avancent l'idée qu'un mécanisme basé sur les projets devrait être inclus dans le cadre global de la REDD (voir la récente communication présentée à la CCNUCC à cet égard par le Paraguay au nom de l'Argentine, du Panama, du Paraguay et du Pérou dans l'Appendice). Les négociations post-2012 au titre du Protocole de Kyoto (Article 3.9) portent aussi sur l'inclusion éventuelle de la REDD dans le mécanisme MDP. Il conviendrait toutefois que les négociateurs reconnaissent que les succès limités des projets de boisement/reboisement $(B / R)$ ne plaident pas en faveur d'une réplication pure et simple du modèle MDP pour l'approche REDD basée sur les projets.

\subsubsection{Approche nationale}

La plupart des propositions présentées par les pays à la CCNUCC préconisent une approche nationale. Cette démarche est le reflet des expériences vécues dans le contexte des approches basées sur les projets souvent caractérisées par des fuites et des coûts de transaction élevés. L'approche nationale aborde aussi les questions liées à la souveraineté. Il y est reconnu que la lutte contre la déforestation implique de procéder à des réformes stratégiques de grande envergure - ce qui relève davantage de l'approche nationale qui est donc potentiellement mieux placée que les deux autres approches (sous-nationale ou combinée) pour aboutir à des réductions qui pourront être réalisées à plus grande échelle et auront un caractère plus permanent.

Les gouvernements adoptant une approche nationale devront établir un système national de capacités MRV et de récompenses pour l'obtention de réductions d'émissions qui seront mesurées en fonction d'un niveau de référence déterminé (voir chapitre 6). Les réductions seront récompensées par l'attribution de crédits carbone commercialisables, des transferts financiers versés par un fonds mondial ou d'autres mécanismes. Dans l'approche nationale, aucun crédit direct ne sera émis internationalement pour récompenser des activités qui se seront déroulées pour réduire des émissions au niveau sous-national.

Afin de leur faciliter l'accès au système international mis en place pour les incitations, chaque pays participant, compte tenu de ses circonstances 
nationales, sera responsable de la mise en œuvre des politiques et mesures (PM) visant à réduire les émissions dues à la déforestation et à la dégradation des forêts sur la totalité de son territoire. Ces PM pourront comporter la mise en place d'un système autorisant l'ouverture de crédits (paiements pour services environnementaux, ou PSE) aux communautés locales. L'un des grands avantages de l'approche nationale tient à ce que les gouvernements peuvent adopter toute une série de stratégies et d'actions axées sur la lutte contre la déforestation et la dégradation des forêts.

\subsubsection{Approche combinée}

$\mathrm{Au}$ vu des circonstances nationales qui diffèrent d'un pays à l'autre, certaines propositions ont été présentées à la CCNUCC pour suggérer d'intégrer les activités menées au niveau sous-national dans un système de comptabilisation à travers une approche combinée (explicitement présentée pour la première fois, par Pedroni et al. 2007). S'ils adoptent cette approche, les pays pourront entreprendre des actions au titre de la REDD au niveau qu'ils souhaiteront. Ceux qui décident de commencer au niveau sous-national pourront ensuite progresser au niveau de l'approche nationale à mesure qu'ils renforcent leurs capacités et améliorent la gouvernance. Il sera obligatoire de passer à l'étape suivante, celle de l'approche nationale, soit en fonction d'un calendrier convenu et déterminé, soit quand un certain pourcentage - reconnu et convenu d'avance - d'un périmètre forestier sera au bénéfice de projets REDD, la première des deux options venant à échéance étant réputée s'appliquer.

Malgré le caractère obligatoire du passage à l'approche nationale, il restera possible de porter au crédit du système de comptabilisation qui sera mis en place les crédits (droits d'émission) acquis au titre de projets individuels. L'approche combinée comporte donc deux aspects singuliers : premièrement, la capacité de passer progressivement d'une approche sous-nationale à une approche nationale dans des délais prescrits ; et, deuxièmement, la possibilité, pour chaque pays, de comptabiliser et recevoir des crédits (droits d'émission) internationaux aux niveaux sous-national et national simultanément (voir Figure 4.1). En outre, différents pays peuvent utiliser différents mécanismes de comptabilisation au même moment.

Dans une approche combinée où les opérations de comptabilisation et d'enregistrement des crédits carbone s'effectuent aux niveaux sous-national et national, il sera nécessaire d'harmoniser les procédures liées aux capacités MRV et à la fixation des niveaux de référence. Un système de partage des crédits entre ces deux niveaux pourrait être instauré sur le modèle du mécanisme de mise en œuvre conjointe (MOC) du Protocole de Kyoto. A la fin de chaque exercice comptable, le pays serait tenu de déduire tous les crédits attribués et engagés au niveau sous-national des crédits attribués au niveau national 
au titre des réductions d'émissions applicables à tout le territoire national (voir Encadré 4.1). Au cas où aucun crédit carbone n'aurait été engrangé au niveau national, la procédure d'attribution des crédits carbone continuerait à s'appliquer aux activités indépendantes dûment validées et vérifiées au niveau sous-national.

\section{Encadré 4.1. Comment une approche combinée pourrait fonctionner}

Un projet génère des réductions d'émissions atteignant 1000 tonnes de dioxyde de carbone pendant l'exercice comptable. La réduction totale des émissions pour le pays (crédits carbone) atteint 5000 tonnes pendant cette même période. Les 1000 tonnes déjà portées au crédit du projet doivent être déduites du solde national. Aux fins de pouvoir éventuellement déduire les coûts du projet liés aux fuites, ainsi qu'à la surveillance, aux rapports de suivi et au contrôle (MRV) et prendre en compte le risque de non-permanence (relèvement possible des taux d'émissions dans le futur), le gouvernement pourrait retenir une certaine partie des crédits carbone attribués à ce projet. Un arrangement pourrait donc être trouvé entre le gouvernement et les responsables du projet aux termes duquel 70 pour cent des crédits attribués reviendraient au projet et 30 pour cent au gouvernement. Dans ce scénario, le projet conserverait 700 crédits et le gouvernement accuserait un total de 4300 crédits.

Même dans le cas d'une approche exclusivement nationale, un pays peut aussi prévoir d'attribuer à des projets une partie des crédits nationaux. Cette disposition permettrait de réduire la déforestation et la dégradation et de compenser les dépenses encourues par les districts, les communautés et les agriculteurs au titre d'activités en faveur de la protection des forêts. En d'autres termes, un pays devrait mettre en place un système national de paiements pour services environnementaux (PSE) qui étendrait la portée du système global au niveau local. Dans une approche combinée, un tel système serait considéré comme faisant partie intégrante de l'accord international alors que ce ne serait pas le cas dans un modèle exclusivement national.

\subsection{Evaluation des trois approches}

Les avantages des trois approches décrites ci-dessus peuvent être évalués en fonction du critère des " $3 \mathrm{E}$ » qui a fait l'objet d'une analyse détaillée au chapitre 2 : Est-ce que le mécanisme atteint ses objectifs en matière d'émissions de gazà effet de serre (efficacité du carbone) ? Est-ce que ces objectifs sont atteints au coût minimum (efficience du coût) ? Quelles en sont les répercussions sur la répartition et quels sont les bénéfices y associés (équité et bénéfices associés) ? Cette section est consacrée à l'évaluation de ces trois critères qui sont récapitulés au Tableau 4.1. 
Tableau 4.1. Avantages et inconvénients des approches infranationales, nationales et combinées

\begin{tabular}{|c|c|c|c|}
\hline \multirow[b]{2}{*}{ Modèle REDD } & \multicolumn{3}{|c|}{ Critères } \\
\hline & Efficacité & $\begin{array}{c}\text { Efficience } \\
\text { (rentabilité) }\end{array}$ & $\begin{array}{c}\text { Equité et bénéfices } \\
\text { associés }\end{array}$ \\
\hline $\begin{array}{l}\text { Approche } \\
\text { infranationale }\end{array}$ & $\begin{array}{l}\text { + Participation élargie } \\
\text { à court terme } \\
+ \text { Intéressant pour les } \\
\text { investisseurs privés } \\
\text { - Fuites sur territoire } \\
\text { national (problème) } \\
\text { - Ne déclenche pas } \\
\text { les changements } \\
\text { politiques requis } \\
\text { - Faible participation } \\
\text { des pays-hôtes }\end{array}$ & $\begin{array}{l} \pm \text { Coûts MRV totaux } \\
\text { en baisse mais } \\
\text { plus élevés par } \\
\text { équivalent } \mathrm{CO}_{2} \\
+ \text { Recours possible } \\
\text { aux paiements } \\
\text { différenciés pour } \\
\text { les incitations: } \\
\text { réduit les coûts }\end{array}$ & $\begin{array}{l}\text { + Facilite la } \\
\text { participation des } \\
\text { pays pauvres et } \\
\text { des pays à faible } \\
\text { gouvernance } \\
\text { + Peut cibler les } \\
\text { groupes les plus } \\
\text { pauvres d'un } \\
\text { pays et favoriser } \\
\text { une plus grande } \\
\text { participation des } \\
\text { communautés }\end{array}$ \\
\hline $\begin{array}{l}\text { Approche } \\
\text { nationale }\end{array}$ & $\begin{array}{l}\text { + Elargissement des } \\
\text { politiques } \\
+ \text { Tient compte des } \\
\text { fuites dans le pays } \\
\text { + Renforcement du } \\
\text { sentiment national } \\
\text { d'appropriation } \\
\text { - Questions non } \\
\text { résolues liées } \\
\text { aux niveaux } \\
\text { de référence } \\
\text { (additionnalité) }\end{array}$ & $\begin{array}{l}\text { + MRV et coûts } \\
\text { de transaction } \\
\text { moins chers par } \\
\text { équivalent } \mathrm{CO}_{2} \\
\text { + Stratégies (non- } \\
\text { PSE) disponibles à } \\
\text { bas prix } \\
\text { - Potentiel d'échec } \\
\text { des politiques et de } \\
\text { la gouvernance }\end{array}$ & $\begin{array}{l}\text { + Transferts globaux } \\
\text { potentiellement } \\
\text { plus importants } \\
\text { + Meilleur aligne- } \\
\text { ment sur les } \\
\text { stratégies de } \\
\text { développement } \\
\text { national } \\
\text { - Favorise les pays à } \\
\text { revenus moyens } \\
\text { - Risque élevé } \\
\text { de capture } \\
\text { des niveaux } \\
\text { et des élites } \\
\text { («nationalisation » } \\
\text { des droits } \\
\text { d'émission) }\end{array}$ \\
\hline $\begin{array}{l}\text { Approche } \\
\text { imbriquée }\end{array}$ & $\begin{array}{l}\text { + Combine les points } \\
\text { forts des deux } \\
\text { autres approches } \\
+ \text { Flexibilité basée sur } \\
\text { les circonstances } \\
\text { nationales } \\
+ \text { Potentiel } \\
\text { d'expansion des } \\
\text { transferts globaux } \\
\text { - Questions non } \\
\text { résolues liées } \\
\text { aux niveaux } \\
\text { de référence } \\
\text { (additionnalité) }\end{array}$ & $\begin{array}{l}\text { + Paiements de } \\
\text { compensation } \\
\text { différenciés et } \\
\text { stratégies élargies } \\
\text { - Coûts MRV élevés } \\
\text { (ventilation exigée } \\
\text { pour les données } \\
\text { nationales) } \\
\text { - Défis liés à } \\
\text { I'harmonisation des } \\
\text { niveaux nationaux } \\
\text { et infranationaux }\end{array}$ & $\begin{array}{l}\text { + Participation } \\
\text { accrue des pays } \\
\text { et transferts plus } \\
\text { importants vers les } \\
\text { pays pauvres } \\
\text { + Possibilité de } \\
\text { cibler les groupes } \\
\text { pauvres de la } \\
\text { population }\end{array}$ \\
\hline
\end{tabular}




\subsubsection{Efficacité}

Dans le contexte de l'efficacité du carbone et des objectifs de réduction des émissions, il est possible de procéder à une évaluation des trois approches (sous-nationale, nationale, et combinée) en les replaçant dans trois dimensions principales : (i) leur capacité à traiter les fuites et l'additionnalité ; (ii) le niveau global de participation qui aura un effet sur le résultat obtenu en matière de réduction globale des émissions ; et (iii) les réformes stratégiques de grande envergure qui auront des effets sur l'ampleur, les coûts et la permanence des réductions d'émissions.

Il est indispensable de fixer des niveaux de référence de comptabilisations crédibles (pour les crédits carbones) dans les approches nationales et de s'attaquer aux questions de la permanence et de la détermination des responsabilités (étudiées au chapitre 8). Les questions liées à ces procédures restent posées et n'ont pas encore été toutes résolues au fil des négociations internationales. Le risque est réel car au vu du très grand nombre de critères qui doivent être discutés pour fixer les niveaux de référence (comme les circonstances nationales, par exemple), il en est qui peuvent être gonflés (surévalués) et donner lieu, par conséquent, à ce qui a été qualifié de "réductions fictives " (correspondant à l'absence d'additionnalité) (voir chapitre 6). Un tel scénario compromettrait à la fois l'efficacité et la crédibilité à long terme des systèmes nationaux.

Le champ géographique d'application des approches nationales et combinées est potentiellement beaucoup plus vaste que celui des approches sous-nationales - ce qui permet à ces deux premières approches de prendre en compte les problèmes liés aux fuites sur le territoire national et d'être plus efficaces ( $M$-Co Consulting, 2008 ; voir aussi le chapitre 7).

La plupart des pays en développement ne peuvent actuellement adopter une approche nationale en raison de l'insuffisance de leur infrastructure en capacités MRV. Cela soulève le problème des fuites au niveau international. La flexibilité de l'approche combinée devrait permettre à la plupart des pays de participer plus tôt à la réduction des émissions puisqu' ils pourraient adopter soit une approche (temporaire) par le biais des projets soit une approche nationale, ou les deux à la fois. Leur choix sera fonction de leurs capacités MRV, qu'ils aient ou non des institutions en place pour gérer les fonds liés à la REDD ainsi que de la nature de leurs stratégies pour réduire les émissions dues à la déforestation et à la dégradation des forêts. La flexibilité de l'approche combinée et ses possibilités d'ouverture en matière de participation devraient favoriser l'obtention de taux d'émission inférieurs à ceux des deux autres approches.

Les investisseurs privés pourraient avoir quelque réticence à faire l'acquisition de réductions d'émissions auprès des pays. Ils pourraient préférer investir dans des projets forestiers " tangibles ", directement associés à des réductions d'émissions et à d'autres bénéfices, tels que la conservation de la biodiversité 
et la réduction de la pauvreté. Ils sont aussi moins enclins à faire des avances de fonds au niveau national au titre des réductions d'émissions qu'à investir directement dans des projets forestiers car ils n'ont que peu de pouvoir de contrôle, voire aucun, sur les risques d'investissements nationaux. Tout cela ne peut qu'exacerber le problème d'une participation déjà limitée des pays.

Des mesures portant, par exemple, sur la réforme du régime foncier et l'amélioration de la gouvernance pourraient être les éléments clé d'une stratégie nationale de réduction des émissions dues à la déforestation et à la dégradation des forêts (REDD). Il pourrait être difficile, cependant, de suivre les effets de ces réformes dans certaines zones géographiques particulières. En outre, ce type de réformes ne relève généralement pas d'une approche sous-nationale ou basée sur les projets. Ce sont donc les approches nationales - plutôt que sous-nationales - qui sont le mieux à même de favoriser l'adoption de grandes politiques stratégiques et d'aboutir à des réductions à la fois plus importantes et de plus longue durée.

\subsubsection{Efficience}

Plusieurs facteurs sont susceptibles d'avoir un impact sur l'efficience des coûts (ou rentabilité) dans le contexte des trois approches (sous-nationale, nationale et combinée) à la REDD, notamment : (i) les coûts liés à la surveillance, aux rapports de suivi et au contrôle (MRV) ; (ii) les coûts de mise en œuvre des stratégies; et (iii) les paiements effectués au titre des coûts d'opportunité.

Toute infrastructure MRV mise en place au niveau national entraîne des économies d'échelle significatives, ce qui indique une forte présomption de plus grande efficience (rentabilité) de l'approche nationale (par rapport aux deux autres approches) en matière de coûts afférents à la réduction d'émissions par unité de réduction d'émission de dioxyde de carbone ou par superficie visée. Une approche exclusivement nationale, par exemple, ne doit pas nécessairement reposer sur une ventilation des données par région ou district, ce qui diminue le nombre de parcelles-échantillons à surveiller. L'approche combinée est plus onéreuse que l'approche nationale parce que la surveillance et la comptabilisation doivent se faire aussi bien au niveau sous-national qu'au niveau national (et que la désagrégation des données présentées au niveau national est un processus coûteux).

Un deuxième élément ayant un effet sur la rentabilité (efficience des coûts) est celui du coût de mise en œuvre d'une stratégie liée à la REDD. Toute mise en œuvre d'un système d'enregistrement des crédits au niveau sous-national (c'est à dire d'un système de PSE) suppose la prise en compte de coûts tels que ceux afférents à l'enregistrement des projets auprès d'institutions centrales, à la validation et à la vérification, ainsi que des frais d'administration des contrats. 
Un système établi au niveau national bénéficiera certes des économies d'échelle et d'un potentiel de réductions d'émissions plus important à un coût plus faible mais la bureaucratie et la corruption peuvent le rendre inopérant - ce qui ne sera pas le cas d'une approche sous-nationale dont les coûts globaux de transaction par unité de réduction d'émissions sont sans doute plus élevés mais dont le fonctionnement devrait être plus efficace. Les approches sousnationales se traduisent souvent par de petits projets administrés par des entités privées qui ont l'expérience des mécanismes du marché du carbone et donnent la priorité à la rentabilité.

Les approches nationales peuvent comporter des réformes stratégiques de grande envergure dont bon nombre seront moins onéreuses à appliquer que les mécanismes de paiements pour des services environnementaux (PSE). Parfois, ces réformes pourraient même être à l'origine d'économies comme, par exemple, en supprimant l'octroi de subventions qui stimulent la déforestation et la dégradation des forêts.

Les coûts d'opportunité liés à la conservation de la forêt (c.-à-d., le manque à gagner résultant le plus souvent des profits non réalisés qui auraient pu être tirés de l'agriculture et de l'exploitation du bois) varient énormément d'un titulaire de droits d'utilisation sur la forêt à l'autre. Si des compensations en rapport avec le manque à gagner (les coûts d'opportunité) qu'ils subissent pouvaient être versées aux titulaires de droits sur la forêt, le coût total de la réduction d'émissions serait moins élevé. Dans une étude faite au Brésil, Börner et Wunder (2008) ont estimé que des paiements parfaitement différenciés - comparés à des compensations de montant uniformisé - permettent d'économiser de 45 à 75 pour cent du coût total de réduction des émissions.

L'adoption de la pratique des paiements différenciés pourrait être plus réaliste dans le cas d'une approche sous-nationale en raison de coûts de transaction plus élevés dans un système national. Cette pratique ne suscite cependant aucun problème du point de vue de l'équité puisque certains des titulaires de droits les plus pauvres sont aussi ceux dont les coûts d'opportunité (le manque à gagner) sont les moins élevés. Le système national des PSE du Costa Rica témoigne des difficultés, notamment en matière de paiements qui ne sont pas rentabilisés, auxquelles donne lieu le fait de ne pas prendre en compte les activités non additionnelles (Karousakis 2007).

\subsubsection{Equité}

L'équité est une question que les pays doivent se poser à l'échelle internationale et à l'intérieur de leur territoire national. Dans ce dernier cas, les contours de la question seront largement déterminés par les politiques et les stratégies nationales en matière de REDD. Bien qu'un accord international sur la 
réduction des émissions dues à la déforestation et à la dégradation des forêts soit en fin de compte probablement " neutre quant à la mise en œuvre ", au sens où il ne spécifiera pas quelles stratégies nationales doivent effectivement être mises en œuvre, ses dispositions globales auront des répercussions sur la répartition des bénéfices et des coûts à l'intérieur du territoire national.

Au niveau international, un accord qui n'accepterait que l'approche nationale pour la REDD pourrait exclure quasiment tous les pays à faibles revenus au motif que leur infrastructure en matière de capacités MRV est inadéquate et leur gouvernance insuffisante. Les courants internationaux d'échanges monétaires se réorienteraient donc vers quelques pays à revenus moyens comme le Brésil. Il apparaît cependant que les approches sous-nationales ne soient pas davantage les meilleures à cet égard, comme le montre l'expérience du MDP. En 2007 en effet, 73 pour cent de tous les crédits MDP vendus étaient « made in China" fabriqués en Chine (Hamilton et al. 2008). Cela montre bien la nécessité - quelle que soit l'approche retenue - de renforcer les capacités et les institutions nationales et, plus généralement, d'améliorer la gouvernance et la responsabilisation pour assurer la participation des pays les plus pauvres.

S'agissant de l'équité dans le pays même, une approche nationale centralisée pourrait restreindre la participation des communautés rurales à l'élaboration et la mise en œuvre de la REDD. Il pourrait en résulter une répartition inéquitable des bénéfices et la "nationalisation » des droits sur le carbone. De gros afflux de fonds pourraient aussi accroître le risque de corruption et être captés par l'Etat, empêchant ainsi les plus pauvres d'avoir accès aux bénéfices. Les façons de procéder des gouvernements sont décidément toujours très mitigées quand il s'agit de promouvoir un processus de prise de décision global pour la REDD (Foti et al. 2008), ce qui ne semble pas indiquer une forte mobilisation pour une large participation du niveau local audit processus. Si les façons de procéder au niveau national sont inéquitables, les résultats risquent de l'être aussi. A contrario, une approche nationale qui s'alignerait sur des stratégies de développement national pourrait être porteuse de bénéfices à long terme pour le développement.

Des approches sous-nationales et combinées - à plus petite échelle - pourraient être plus flexibles que des approches nationales à plus grande échelle, et mieux adaptées pour répondre à des besoins spécifiques. Des éléments de réponse nous sont apportés à cet égard par certains projets forestiers utilisateurs de crédits carbones; il semble que de tels projets aient permis de renforcer les capacités locales, la prise de décision participative et une gestion communautaire des ressources (Corbera 2005). Il faut reconnaitre cependant que les façons de procéder des investisseurs privés et des ONG de protection environnementale sont très mitigées quand il s'agit de tenir compte des préoccupations de la communauté et de les inclure dans leurs projets. Ce sont des objectifs de protection du climat mondial qui gouvernent en premier lieu les marchés du 
carbone, plutôt que des objectifs socio-économiques à caractère local. Les deux approches (sous-nationale et nationale) seront vraisemblablement confrontées à des défis dans ce domaine quoique les facteurs primordiaux influençant le niveau et les formes de participation ainsi que les acteurs et processus impliqués dans ces deux approches soient très différents.

\subsection{Résumé et conclusions}

Trois façons d'aborder (approches) la question de l'échelle ou du niveau géographique des systèmes de comptabilisation et d'incitations envisagés pour la REDD ont été étudiées dans ce chapitre : le soutien direct aux projets (niveau sous-national), le soutien direct aux pays (niveau national), ou une approche combinée, mélange des deux précédentes approches.

Une approche sous-nationale ou approche basée sur les projets permet une participation anticipée et plus large, et retient l'intérêt des investisseurs privés. Elle est cependant plus vulnérable aux fuites (augmentation des émissions à l'extérieur du périmètre du projet) et ne peut traiter les facteurs déterminants de la déforestation et de la dégradation des forêts.

Une approche nationale permet d'élaborer et de mettre en œuvre toute une série de stratégies, de s'attaquer au problème des fuites sur le territoire national et permet aussi l'appropriation au niveau national. A court ou moyen terme toutefois, la question de la faisabilité de cette approche se pose pour un grand nombre de pays qui n'ont pas les moyens de l'adopter rapidement. Elle est plus vulnérable à des fautes ou des échecs de gouvernance et elle est moins susceptible de mobiliser l'intérêt des investisseurs privés ou la participation des gouvernements locaux.

L'approche combinée est la plus flexible. Elle permet aux pays de démarrer des activités au niveau sous-national et de passer progressivement à une approche nationale. L'approche combinée permet aux deux approches (sous-nationale et nationale) de coexister au sein d'un système dans lequel les crédits REDD sont attribués à la fois au titre des projets et par le gouvernement, optimisant ainsi le potentiel des approches sous-nationale et nationale. Le défi à relever dans le cas d'une approche combinée cependant consiste précisément à harmoniser ces deux niveaux de fonctionnement. 


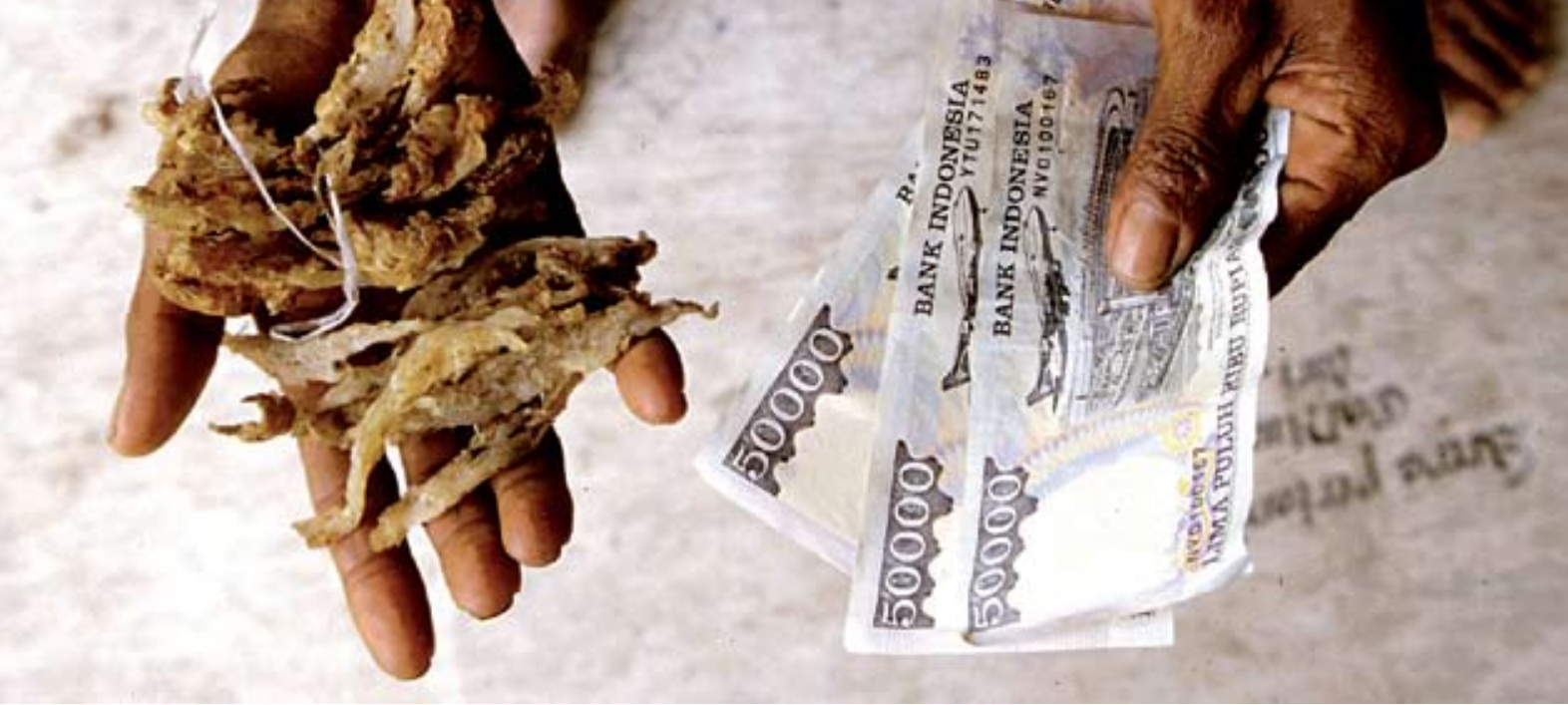

\section{Chapitre 5}

\section{Comment ajuster les besoins des pays aux sources de financement?}

Michael Dutschke et Sheila Wertz-Kanounnikoff

Avec Leo Peskett, Cecilia Luttrell, Charlotte Streck et Jessica Brown

\subsection{Le défi : Mécanismes efficaces pour faire face à la diversité des situations}

La réduction des émissions dues à la déforestation et à la dégradation des forêts (REDD) est une option potentiellement peu onéreuse pour atténuer les effets du changement climatique à condition de passer à l'action dès aujourd'hui (Stern 2006). Si les crédits carbone forestiers sont inclus dans les échanges globaux d'émissions, le coût en résultant pour réduire de moitié les émissions globales nettes de dioxyde de carbone d'ici 2030 est estimé de 17 à 33 milliards de dollars US par an (Eliasch 2008). La treizième Conférence des Parties (COP 13) de la Convention-cadre des Nations Unies sur les changements climatiques (CCNUCC) en 2007 a jeté les bases de l'inclusion de la REDD dans les pays en développement dans le régime post-2012 de protection du climat. Les pays développés sont encouragés à trouver les moyens nécessaires pour financer ces activités REDD dans les pays en développement.

Les capacités de réduction des émissions forestières varient d'un pays en développement à l'autre. Ces variations sont dues à des conditions nationales différentes liées à des facteurs déterminants différents de déforestation et de 
dégradation des forêts, ainsi qu'à des capacités institutionnelles différentes de surveillance, de contrôle et de réglementation.

De multiples sources de financement REDD existent déjà et sont disponibles ou susceptibles de le devenir. Le montant et la composition de ces fonds dépendent du modèle qui sera retenu pour la REDD et varieront dans le temps. Actuellement, la plus grande partie des fonds REDD est spécifiquement affectée au renforcement des capacités ou capacité de réponse. Bien que la nature du mécanisme REDD continue à faire l'objet de débats dont les résultats se répercuteront sur les besoins de financement et les mouvements de capitaux, nous présentons ci-après les résultats de notre étude préliminaire sur les flux potentiels dans différents contextes nationaux et identifions les lacunes possibles en matière de financement.

\subsection{Quels sont les besoins en matière de financement?}

Indépendamment du modèle qui sera retenu pour le mécanisme REDD (Eliasch 2008, voir Tableau 5.1), deux besoins principaux existent en matière de financement :

- Financement des activités de renforcement des capacités par avance de fonds (capacité de réponse) : Les pays doivent satisfaire à des conditions minimales d'actualisation de leurs capacités comme : de mettre en place l'infrastructure nécessaire à la surveillance de la réduction des émissions, de préciser la situation en matière de régime foncier et de renforcer les capacités institutionnelles d'application des législations. Une étude estime les couts étalés sur une période de cinq ans, de renforcement des capacités pour 40 nations forestières à un montant pouvant atteindre jusqu'à 4 milliards de dollars US (Hoare et al. 2008). Les montants en cause et types de coûts varieront de façon significative d'un pays à l'autre.

- Financement lié aux dépenses de fonctionnement de la REDD : Les coûts liés à la réduction des émissions relèvent de deux catégories : les coûts liés à la protection des forêts et les coûts d'opportunité. La première catégorie concerne les coûts de mise en œuvre des politiques et mesures (PM) nécessaires, à l'intérieur et à l'extérieur du secteur forestier, pour réduire les émissions des forêts. Des exemples sont, notamment : la surveillance des forêts, la réforme des régimes fonciers, l'application des législations, la taxation des terres forestières, l'imposition de restrictions en matière de construction de routes et de zonage agricole. La deuxième catégorie est constituée par les coûts d'opportunité qui correspondent au manque à gagner que représente la privation de bénéfices liés à des activités de déforestation ou les frais encourus au titre de l'adoption de stratégies 
Tableau 5.1. Résumé des besoins de financement de la REDD

\begin{tabular}{|c|c|c|c|}
\hline & \multirow{2}{*}{$\begin{array}{c}\text { Avance de fonds } \\
\text { (renforcement des } \\
\text { capacités) }\end{array}$} & \multicolumn{2}{|c|}{$\begin{array}{l}\text { Dépenses de fonctionnement } \\
\text { (réduction des émissions) }\end{array}$} \\
\hline & & $\begin{array}{l}\text { Coûts liés à la } \\
\text { protection des } \\
\text { forêts }\end{array}$ & $\begin{array}{l}\text { Coûts } \\
\text { d'opportunité }\end{array}$ \\
\hline Objectifs & $\begin{array}{l}\text { Investissements initiaux } \\
\text { pour mettre en place } \\
\text { une infrastructure REDD } \\
\text { (systèmes de surveillance } \\
\text { données forestières et stock } \\
\text { de carbone), et promouvoir } \\
\text { la participation } \\
\text { des parties prenantes }\end{array}$ & $\begin{array}{l}\text { Couvrir les } \\
\text { coûts liés à la } \\
\text { mise en œuvre } \\
\text { des politiques } \\
\text { et mesures } \\
\text { (PM) faisant } \\
\text { fructifier les } \\
\text { investissements } \\
\text { REDD }\end{array}$ & $\begin{array}{l}\text { Compenser } \\
\text { le manque à } \\
\text { gagner dû aux } \\
\text { activités liées } \\
\text { à la réduction } \\
\text { des émissions } \\
\text { provenant } \\
\text { des forêts }\end{array}$ \\
\hline $\begin{array}{l}\text { Eléments } \\
\text { constitutifs }\end{array}$ & $\begin{array}{l}\text { - Financement initial } \\
\text { - Peu d'effet direct sur } \\
\text { les émissions liées à } \\
\text { l'utilisation des terres } \\
\text { - Coûts de transaction } \\
\text { initiaux }\end{array}$ & $\begin{array}{l}\text { - Financement } \\
\text { initial } \\
\text { - Coûts et } \\
\text { bénéfices } \\
\text { dépendent } \\
\text { des stratégies } \\
\text { adoptées } \\
\text { - Coûts de } \\
\text { transaction } \\
\text { récurrents }\end{array}$ & $\begin{array}{l}\text { - Financement } \\
\text { continu } \\
\text { - Les coûts } \\
\text { varient dans } \\
\text { l'espace et dans } \\
\text { le temps }\end{array}$ \\
\hline Exemples & $\begin{array}{l}\text { - Mise en place d'un } \\
\text { système de surveillance } \\
\text { (0.5 à } 2 \text { millions de dollars } \\
\text { US en Inde et au Brésil) } \\
\text { - Compilation d'inventaires } \\
\text { forestiers ( } 50 \text { millions } \\
\text { dollars US pour } 25 \\
\text { nations) } \\
\text { - Renforcement des } \\
\text { capacités ( } 4 \text { milliards de } \\
\text { dollars US pour } 40 \text { nations } \\
\text { pendant } 5 \text { ans) } \\
\text { - Réforme des régimes } \\
\text { fonciers (en fonction de } \\
\text { la taille, } 4 \text { à } 20 \text { milliards } \\
\text { de dollars US pendant } 5 \\
\text { ans par pays sur la base } \\
\text { d'estimations établies } \\
\text { pour le Rwanda, le Ghana } \\
\text { et les lles Salomon) }\end{array}$ & $\begin{array}{l}\text { - Coûts } \\
\text { récurrents pour } \\
\text { inventaires } \\
\text { forestiers (7-17 } \\
\text { millions de } \\
\text { dollars US par } \\
\text { an pour } 25 \\
\text { pays) } \\
\text { - Suivi lié à } \\
\text { l'application } \\
\text { des législations }\end{array}$ & $\begin{array}{l}\text { - Coûts } \\
\text { d'opportunité } \\
\text { pour réduire } \\
\text { de moitié la } \\
\text { déforestation } \\
\text { (7 milliards de } \\
\text { dollars US par } \\
\text { an pendant } \\
30 \text { ans pour } \\
\text { huit pays) }^{c}\end{array}$ \\
\hline
\end{tabular}

${ }^{a}$ Hoare et al. $2008 ;{ }^{b}$ Eliasch $2008 ;$; Grieg-Gran 2008 
d'exploitation forestière plus durables. Ces coûts varient d'un endroit à l'autre et dans le temps. Les coûts d'opportunité sont plus élevés quand les marchés sont accessibles et quand l'expansion des activités de protection de la forêt (par exemple, avec la REDD) entraîne une intensification de l'agriculture. Des coûts d'opportunité peu élevés n’impliquent cependant pas nécessairement que les actions engagées au titre de la REDD seront peu onéreuses puisque ces actions se déroulent souvent dans des zones regroupant les plus grands défis en matière de politique, d'administration et de surveillance forestières (Eliasch 2008).

\subsection{Le contexte forestier a un effet sur les besoins de financement}

Les pressions exercées sur les forêts varient d'un pays et d'une région à l'autre, ainsi que dans le temps. La pression anthropique sur les forêts est modelée, entre autres, par l'accès au marché, les types d'exploitation forestière et la sécurité foncière. Chomitz et al. (2006) ont établi une typologie stylisée des forêts en trois parties : le "massif forestier " (ou zone centrale) au-delà des limites des terres agricoles (ou " frontière agricole »), la " frange forestière » et les zones litigieuses, ainsi que les " mosaïques " dans lesquelles coexistent terres forestières et terres agricoles (Tableau 5.2). Ces trois types de forêts correspondent essentiellement aux trois stades de la courbe de transition des forêts (Figure 5.1).

On estime que les fronts de déforestations dans lesquelles la déforestation est concentrée, sont actuellement répartis de façon assez uniforme entre toutes les régions (Figure 5.2). Différentes stratégies pourraient être requises pour relever les défis liés à la gouvernance ainsi qu'à la déforestation et à la dégradation, dans différents types de forêts. Des stratégies, par exemple, qui pourraient être importantes pour améliorer la gestion forestière dans les mosaïques de terres forestières - dans lesquelles la dégradation est concentrée - pourraient inclure le respect des droits de propriété foncière et la création de nouveaux marchés pour les services environnementaux (Tableau 5.2). 
Table 5.2. Trois types stylisés de forêts.

\begin{tabular}{|c|c|c|c|}
\hline & $\begin{array}{l}\text { "Cœur » des } \\
\text { forêts, au-delà } \\
\text { de la « frontière } \\
\text { agricole " } \\
\text { (approx. } 49 \% \text { de } \\
\text { la superficie des } \\
\text { forêts tropicales) }\end{array}$ & $\begin{array}{l}\text { Lisières des } \\
\text { forêts et zones } \\
\text { litigieuses } \\
\text { (approx. } 37 \% \text { de } \\
\text { la superficie des } \\
\text { forêts tropicales) }\end{array}$ & $\begin{array}{l}\text { Terres « mosaïques » } \\
\text { (approx. } 14 \% \text { de la } \\
\text { superficie des forêts } \\
\text { tropicales) }\end{array}$ \\
\hline Caractéristiques & $\begin{array}{l}\text { - Eloignées des } \\
\text { marchés; faible } \\
\text { déforestation } \\
\text { - Faible densité de } \\
\text { population mais } \\
\text { pourcentages } \\
\text { élevés de } \\
\text { populations } \\
\text { autochtones et } \\
\text { de populations } \\
\text { pauvres }\end{array}$ & $\begin{array}{l}\text { - Rapide } \\
\text { expansion de } \\
\text { l'agriculture } \\
\text { et forte } \\
\text { déforestation } \\
\text { - Rapide } \\
\text { augmentation de } \\
\text { la valeur foncière } \\
\text { des terres (en } \\
\text { limite de la } \\
\text { "frontière ») } \\
\text { - Conflits liés à } \\
\text { l'utilisation des } \\
\text { forêts (zones } \\
\text { litigieuses) }\end{array}$ & $\begin{array}{l}\text { - Forêts décimées, } \\
\text { fragmentées; } \\
\text { ralentissement de la } \\
\text { déforestation, mais } \\
\text { dégradation plus } \\
\text { importante } \\
\text { - Valeurs foncières } \\
\text { élevées et fortes } \\
\text { densités de } \\
\text { population dont } \\
\text { une bonne partie } \\
\text { vivant dans } \\
\text { les forêts }\end{array}$ \\
\hline $\begin{array}{l}\text { Besoins en } \\
\text { matière de } \\
\text { stratégies }\end{array}$ & $\begin{array}{l}\text { - Protection } \\
\text { des droits } \\
\text { des peuples } \\
\text { autochtones } \\
\text { - Fixation de } \\
\text { droits équitables } \\
\text { pour prévenir } \\
\text { toute expansion } \\
\text { désordonnée } \\
\text { au-delà des } \\
\text { limites des terres } \\
\text { agricoles } \\
\text { - Réglementation } \\
\text { de l'expansion } \\
\text { des } \\
\text { infrastructures }\end{array}$ & $\begin{array}{l}\text { - Application des } \\
\text { règlements et } \\
\text { législations, } \\
\text { par ex. pour } \\
\text { prévenir toute } \\
\text { action tendant } \\
\text { à accaparer des } \\
\text { ressources } \\
\text { - Règlement } \\
\text { équitable des } \\
\text { différends } \\
\text { - Maîtrise de } \\
\text { l'expansion de } \\
\text { l'infrastructure } \\
\text { routière }\end{array}$ & $\begin{array}{l}\text { Faire respecter la } \\
\text { législation sur les } \\
\text { droits de propriété } \\
\text { applicables } \\
\text { aux ressources } \\
\text { naturelles } \\
\text { - Promouvoir les } \\
\text { marchés pour } \\
\text { les services } \\
\text { environnementaux } \\
\text { - Réformer la } \\
\text { règlementation } \\
\text { existante afin } \\
\text { d'encourager les } \\
\text { initiatives dans le } \\
\text { secteur forestier }\end{array}$ \\
\hline
\end{tabular}

Source : Chomitz et al. 2006

\subsection{Ajuster besoins et financement}

Les sources du financement existant et potentiel d'activités liées à la REDD peuvent être d'origine publique ou d'origine privée (Tableau 5.3) et il conviendra de respecter l'adéquation entre les différentes sources de financement et les différents besoins auxquelles elles devront répondre (Tableau 5.4). L'Organisation de coopération et de développement économiques (OCDE), par exemple, ne classe pas les dépenses publiques d'acquisition de crédits carbone sous la rubrique 


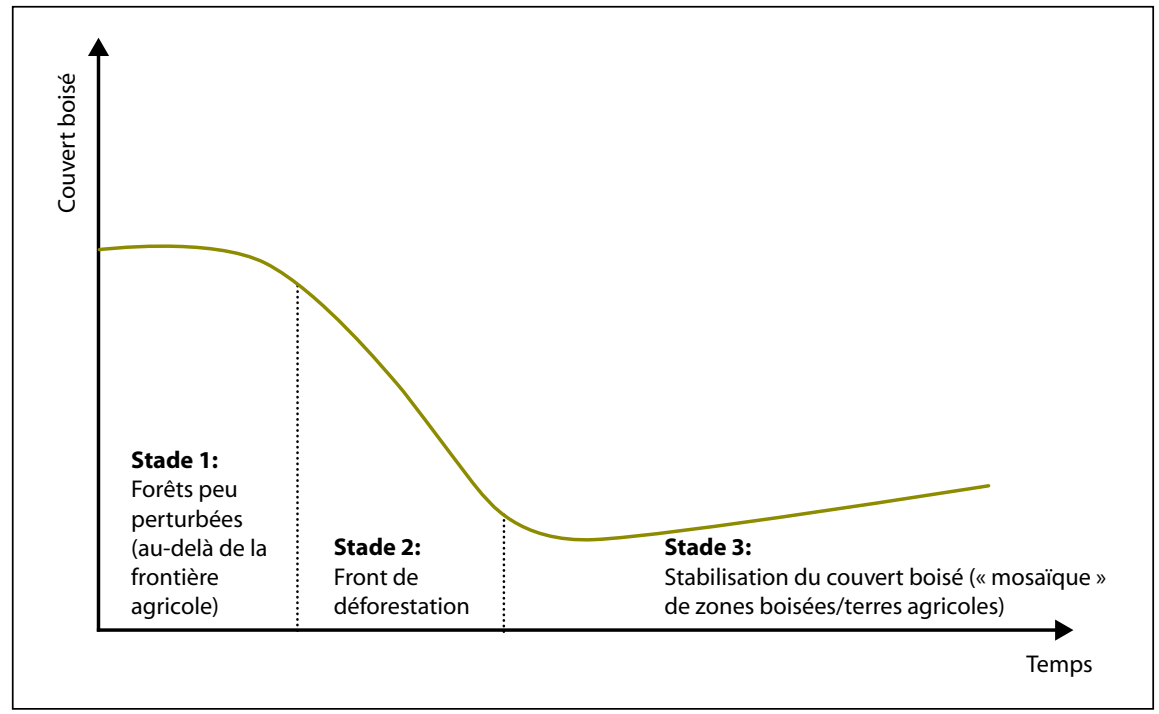

Figure 5.1. Courbe de transition des forêts ou FT (Forest transition)

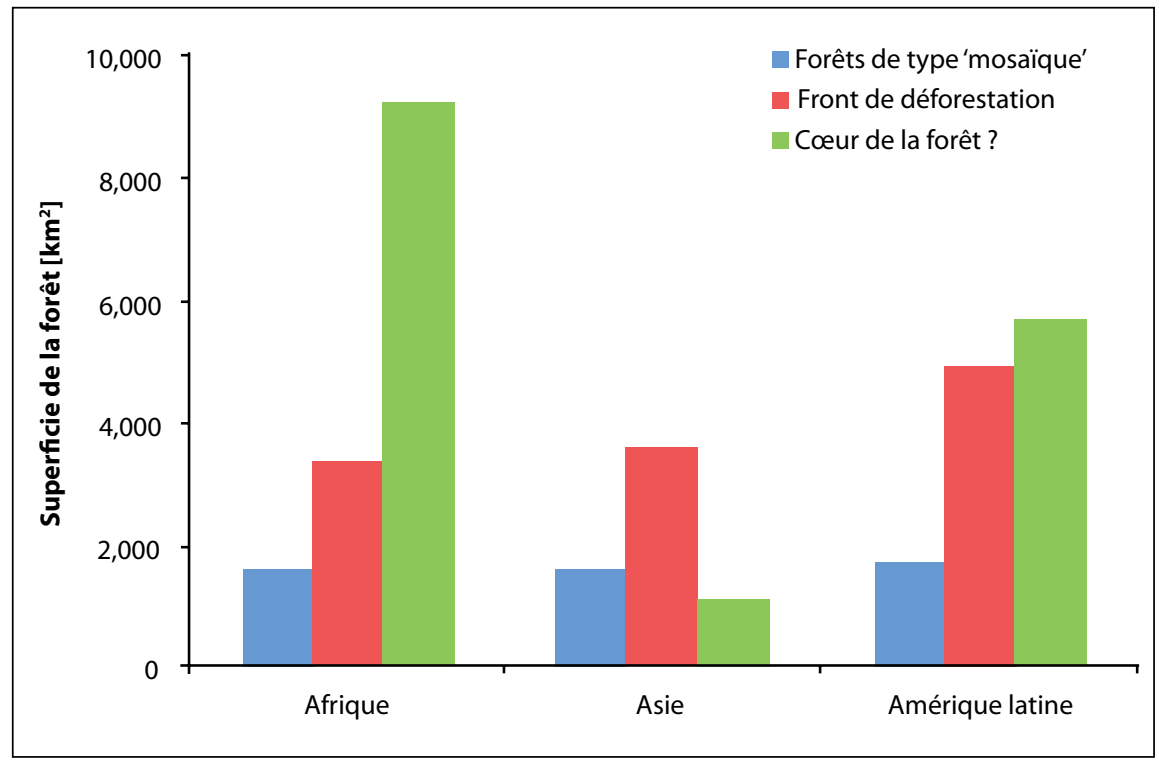

Figure 5.2. Répartition approximative des types de forêts par région Note : Le recours à des approximations est justifié par le fait qu'il est impossible de cartographier des types stylisés de forêts. S'agissant des « mosaïque forestières », seules les données concernant la portion boisée ont été utilisées.

Source : Chomitz et al. (2006) sur la base de données globales de couverture terrestre datant de 2000 (ECJRC 2003) 
de l'aide publique au développement (APD). La raison en est que ces crédits seraient pris en compte comme reports d'APD (c.à-d. qu'ils devraient être soustraits des montants correspondant à l'APD l'année même où ils seraient effectifs) (Dutschke et Michaelowa 2006). Les activités génératrices de crédits carbones devront, par conséquent, être financées à travers le secteur privé et les ventes de crédits REDD aux gouvernements des pays visés à l'Annexe I afin de compenser les émissions de gaz à effet de serre (GES) de ces pays.

La répartition des modes de financement public et privé se fait en fonction de types de forêts bien distincts (Tableau 5.4). Le financement public est particulièrement bien adapté aux forêts qui se situent au-delà des limites des

Table 5.3. Sources potentielles de financement des activités REDD

\begin{tabular}{|c|c|}
\hline \multicolumn{2}{|r|}{ Financement en provenance du secteur public } \\
\hline Type & Description \\
\hline $\begin{array}{l}\text { APD } \\
\text { traditionnellement } \\
\text { liée à la foresterie }\end{array}$ & $\begin{array}{l}\text { - En augmentation ; s'est accrue de 47,6 \% depuis } 2000 \text { et } \\
\text { a totalisé presque } 2 \text { milliards de dollars US entre } 2005 \text { et } \\
2007 \text { (Banque Mondiale 2008) } \\
\text { - Accorde des subventions, des prêts à taux réduits, } \\
\text { des financements à court terme pour des projets } \\
\text { spécifiques et à long terme pour des programmes ou un } \\
\text { soutien budgétaire } \\
\text { - Intéresse aussi les bénéfices associés liés à la réduction } \\
\text { de la pauvreté, la conservation de la biodiversité et } \\
\text { l'amélioration de la gouvernance }\end{array}$ \\
\hline $\begin{array}{l}\text { Nouvelle APD pour } \\
\text { la REDD }\end{array}$ & $\begin{array}{l}\text { - Récente émergence de nouveaux mécanismes de } \\
\text { financement liés à la REDD qui tirent une partie ou la } \\
\text { totalité de leurs revenus de sources internationales de } \\
\text { financement public } \\
\text { - Inclut des financements visant à « relancer» le secteur } \\
\text { privé, comme le Fonds de partenariat de la Banque } \\
\text { mondiale pour le carbone forestier (FCPF), et des sources } \\
\text { de financement visant à renforcer les capacités du secteur } \\
\text { public comme le Fonds du Bassin du Congo }\end{array}$ \\
\hline $\begin{array}{l}\text { Intérieur (niveau } \\
\text { national) }\end{array}$ & $\begin{array}{l}\text { - Financement limité affecté par le secteur public à la } \\
\text { foresterie à partir des taxes et redevances perçues à ce titre } \\
\text { - Type de financement habituel des subventions et } \\
\text { autres incitations } \\
\text { - Parraine les services environnementaux dans les forêts }\end{array}$ \\
\hline
\end{tabular}

Financement en provenance du secteur privé et du marché du carbone (inclut les achats de crédits REDD - par les gouvernements des pays de l'Annexe I - en tant que paiements de compensation des marchés du carbone)

$\begin{array}{ll}\text { Marchés du } & \text { - } \\ \text { carbone existants } & \text { réglementaire actuel exclut la REDD) } \\ \text { - } & \text { Le marché réglementaire se limite au boisement/ } \\ & \text { reboisement (B/R) au titre du MDP, qui peut - ou non - } \\ & \text { faire partie d'un futur mécanisme REDD } \\ \text { - } & \text { Le marché volontaire est dominant dans le secteur } \\ & \text { forestier, représentant jusqu'à } 18 \% \text { de tous les projets dans } \\ & \text { le monde en } 2007 \text { (Hamilton et al. 2007) }\end{array}$




\begin{tabular}{|c|c|}
\hline \multicolumn{2}{|r|}{ Financement en provenance du secteur public } \\
\hline Type & Description \\
\hline $\begin{array}{l}\text { Marchés du } \\
\text { carbone futurs }\end{array}$ & $\begin{array}{l}\text { - Trois grands axes de discussion : } \\
\text { i) intégrer la REDD dans un marché réglementaire global } \\
\text { du carbone ; } \\
\text { ii) attribuer le montant des recettes résultant des enchères; } \\
\text { iii) attribuer le montant des recettes provenant d'autres } \\
\text { redevances, amendes et taxes } \\
\text { - Les marchés intérieurs et régionaux peuvent aussi } \\
\text { envisager d'utiliser le système de comptabilisation des } \\
\text { crédits REDD pour se mettre en règle : par ex. le système de } \\
\text { quotas d'émissions (ETS) de l'Union Européenne }\end{array}$ \\
\hline $\begin{array}{l}\text { Investissements } \\
\text { étrangers directs }\end{array}$ & $\begin{array}{l}\text { Pourraient constituer une source de financement } \\
\text { importante, mais les investissements seraient concentrés } \\
\text { dans des pays à faibles risques dotés d'industries } \\
\text { forestières rentables } \\
\text { - Les investissements dans le secteur forestier ont augmenté } \\
\text { de } 29 \% \text { passant de } 400 \text { millions de dollars US entre } 2000 \\
\text { et } 2002 \text { à } 516 \text { millions de dollars US entre } 2005 \text { et } 2007 \\
\text { (Banque Mondiale 2008) }\end{array}$ \\
\hline $\begin{array}{l}\text { Intérieur (niveau } \\
\text { national) }\end{array}$ & $\begin{array}{l}\text { Partenariats mixtes (public-privé) ou projets de } \\
\text { microcrédits qui ne seront probablement pas significatifs, } \\
\text { notamment dans les pays les moins avancés au regard du } \\
\text { faible niveau de ressources dans ces pays, du manque de } \\
\text { compétences et des difficultés liées à une recherche de } \\
\text { financement auprès de banques nationales peu disposées } \\
\text { à prendre des risques }\end{array}$ \\
\hline Non-lucratif & $\begin{array}{l}\text { - Représente une part croissante du financement } \\
\text { international privé } \\
\text { - Type de financement de petite envergure généralement } \\
\text { accordé sous forme de subventions très spécifiques; } \\
\text { pourrait ne pas être largement applicable à la REDD } \\
\text { - Le secteur non-lucratif s'intéresse à la REDD et pourrait être } \\
\text { moins hostile à la prise de risques que des entreprises à } \\
\text { but lucratif }\end{array}$ \\
\hline
\end{tabular}

terres agricoles, c'est-à-dire " au-delà de la frontière agricole " et aux fronts de déforestation dont les régimes fonciers et les structures de gouvernance en place sont relativement fragiles. Le secteur privé pourrait jouer un plus grand rôle dans le financement d'activités axées sur " mosaïques forestières » dotées de régimes fonciers relativement solides et de bonnes structures de gouvernance. Il y a lieu de noter cependant que ce type de "mosaïques forestières " représente actuellement le plus faible pourcentage de la superficie des forêts tropicales. 


\subsection{Financement par le secteur public}

Un financement public avec mise de fonds d'avance est nécessaire pour créer un climat politique propice à la réalisation de résultats efficaces en matière de REDD, tout particulièrement dans des contextes de faible gouvernance. Un financement à travers l'APD se révèle crucial pour couvrir les dépenses afférentes au renforcement des capacités. Peu de pays en développement ont montré

Tableau 5.4. Adéquation entre sources de financement et types de forêts

\begin{tabular}{|c|c|c|c|}
\hline & $\begin{array}{l}\text { Forêts situées au-delà } \\
\text { des terres agricoles } \\
\text { (de la « frontière } \\
\text { agricole ») }\end{array}$ & $\begin{array}{l}\text { Fronts de } \\
\text { déforestation }\end{array}$ & $\begin{array}{l}\text { "Mosaïques } \\
\text { forestières " }\end{array}$ \\
\hline $\begin{array}{l}\text { Financement } \\
\text { par le secteur } \\
\text { public }\end{array}$ & $\begin{array}{l}\text { Besoin substantiel de } \\
\text { sources internationales } \\
\text { et nationales de } \\
\text { financement }\end{array}$ & $\begin{array}{l}\text { Important pour } \\
\text { faciliter les } \\
\text { investissements liés } \\
\text { à la REDD }\end{array}$ & $\begin{array}{l}\text { Le besoin de } \\
\text { financement } \\
\text { dépendra du } \\
\text { contexte de } \\
\text { gouvernance }\end{array}$ \\
\hline $\begin{array}{l}\text { Financement } \\
\text { par le secteur } \\
\text { privé }\end{array}$ & $\begin{array}{l}\text { Moins probable, des } \\
\text { titres de propriété bien } \\
\text { établis étant requis } \\
\text { pour effectuer des } \\
\text { paiements liés à la } \\
\text { REDD }\end{array}$ & $\begin{array}{l}\text { Probable, à } \\
\text { condition d'avoir } \\
\text { mis en place } \\
\text { des garanties } \\
\text { suffisantes pour } \\
\text { les paiements qui } \\
\text { seront effectués au } \\
\text { titre de la REDD }\end{array}$ & $\begin{array}{l}\text { Très probable, à } \\
\text { condition d'avoir } \\
\text { mis en place } \\
\text { des garanties } \\
\text { suffisantes pour } \\
\text { les paiements qui } \\
\text { seront effectués } \\
\text { au titre de la REDD }\end{array}$ \\
\hline
\end{tabular}

qu'ils avaient soit les moyens soit la volonté politique nécessaire pour financer cet aspect de la REDD. Même si la REDD devait être intégrée dans le marché global du carbone, il faudrait encore trouver un financement supplémentaire de 11 à 19 millions de dollars US par an en provenance d'autres sources selon toute vraisemblance, l'APD - pour réduire de moitié les émissions d'ici 2020 (Eliasch 2008).

L'intérêt croissant manifesté par les bailleurs pour la REDD a donné un coup d'accélérateur à la réorientation des montants disponibles au titre de l'APD vers le secteur des activités forestières basées sur le carbone. Un soutien apporté aux programmes ou aux budgets contribue au renforcement des institutions gouvernementales et permet aux pays de mieux s'approprier les systèmes liés à la REDD. Quand les rentrées dues au carbone sont suffisantes pour servir de garanties, le financement peut prendre la forme d'emprunts. Le soutien apporté au renforcement des capacités peut être acheminé à travers le volet assistance technique de l'APD. 
Mais l'APD n'est sans doute qu'une solution valant pour le court terme; le récent accroissement des montants consacrés par l'APD aux activités liées à la foresterie qui ont atteint presque 2 milliards de dollars US (2005-2007) ne représente qu'une petite fraction des 11 à 19 milliards de dollars US recommandés dans le rapport Eliasch. Il s'ensuit la nécessité d'un déploiement stratégique de l'APD pour stimuler et compléter les investissements privés - ce qui revient essentiellement à appuyer les investissements nécessaires à la mise en place des infrastructures de base. Un apport de fonds permettant d'avoir un effet de levier sur les investissements privés se révèle aussi particulièrement important dans les pays à hauts risques où il n'existe que peu d'opportunités de financement par le secteur privé. Cependant, le financement des activités forestières par l'APD a tendance à graviter vers des environnements moins risqués comme l'Asie du Sud et du Sud-est et les Amériques plutôt que vers l'Afrique (Banque Mondiale 2008).

La probable dépendance de la REDD sur l'APD, en particulier dans le contexte de la création de nouveaux fonds internationaux pour appuyer les activités de la REDD, suscite un certain nombre d'appréhensions liées à la façon dont ces efforts devraient être structurés, notamment :

- le manque d'harmonisation entre les diverses initiatives, ce qui pourrait entraîner des charges supplémentaires pour des gouvernements dont les ressources sont déjà extrêmement sollicitées ;

- le manque d'alignement sur les systèmes administratifs des pays et la faible capacité des gouvernements à absorber les fonds pour en faire un usage efficace ;

- le risque de détourner l'APD d'autres secteurs, comme la santé et l'éducation.

Ces craintes liées au manque d'harmonisation et d'alignement reprennent les mêmes préoccupations exprimées par toutes les parties prenantes au secteur de l'aide qui ont abouti à la Déclaration de Paris sur l'efficacité de l'aide (OCDE 2005).

\subsection{Financement du marché du carbone}

L'investissement dans le marché du carbone est susceptible de mobiliser un intérêt encore plus grand et à plus long terme que celui manifesté par l'APD pour la REDD, compte tenu en particulier de l'incitation que constituent les échanges de crédits carbone sur les marchés de compensation des émissions de GES. Les investissements qui seront effectués dans le marché du carbone seront plus vraisemblablement axés sur les pays dotés de solides structures de gouvernance et de systèmes fonciers bien établis. L'existence de systèmes de vérification ou de certification nationaux est aussi susceptible d'attirer les 
investisseurs. La mesure dans laquelle le secteur privé participera au financement dépend d'un certain nombre de facteurs, notamment :

- des engagements à long terme pris en matière de réduction d'émissions GES ;

- des crédits carbones attribués au titre des approches sous-nationales dans le projet REDD ;

- des actions anticipées génératrices de crédits REDD qui pourront être comptabilisées en prévision de la réalisation des objectifs de l'après 2012.

Le marché volontaire du carbone est un terrain d'expérimentation utile pour les différentes approches de la REDD mais ne sera probablement pas suffisamment rentable pour pouvoir financer de grandes initiatives dans le cadre de la réduction des émissions dues à la déforestation et à la dégradation des forêts. Des mécanismes de financement émergents comme les FFB (Forest Backed Bonds) - instruments financiers commercialisables garantis par des actifs forestiers - pourraient aussi constituer de nouvelles sources d'apport de capitaux (Petley 2007).

Les marchés internationaux du carbone sont une source de financement attrayante pour la REDD parce qu'ils sont potentiellement capables de mobiliser des montants importants pouvant être investis à long terme. Les principales options actuellement débattues dans le contexte de la REDD sont les suivantes : (i) intégration de la REDD dans un marché global du carbone ; (ii) affectation du produit des enchères à un fonds REDD ; et (iii) attribution des recettes provenant d'autres redevances, amendes et taxes à un fonds REDD.

\subsubsection{Intégration de la REDD dans des marchés du carbone globaux}

Le plus grand potentiel de financement pour la REDD est à rechercher dans la création de mécanismes de marché du carbone qui permettent de convertir des réductions d'émissions (provenant d'initiatives REDD) en crédits carbone que les entreprises et les pays pourront utiliser aux fins de respecter les engagements pris en matière d'émissions.

Les montants générés par les crédits commercialisables de la REDD dépendront d'un certain nombre de facteurs. Au nombre de ceux-ci figurent l'ampleur des budgets d'émissions visés par l'Annexe I, la fongibilité des crédits REDD sur les marchés du carbone et d'autres aspects relevant de l'architecture de la REDD. Par fongibilité on entend le type et le degré d'intégration de la REDD dans les marchés du carbone existants. D’aucuns craignent qu'une fongibilité sans réserve inonde les marchés du carbone de crédits REDD, supposant donc que 
ces crédits seront moins onéreux que les crédits provenant d'autres activités d'atténuation. Il n'en sera pas forcément ainsi (voir chapitre 3). A certains égards, une offre excédentaire de crédits carbone bon marché pourrait faire baisser les prix du carbone et libérer les incitations pour les affecter à d'autres activités REDD mais, à d'autres égards, le fait d'accepter les crédits REDD en tant qu'instrument de réglementation crée une demande de nouvelles activités de réduction d'émissions dues à la déforestation et à la dégradation des forêts. Une étude a montré que les crédits REDD, même en supposant acquise la condition d'un accès au marché sans restrictions, n'engloutirait très probablement pas le marché du carbone. Laisser entrer tous les crédits forestiers sur le marché ne devrait vraisemblablement avoir pour seul effet que de faire diminuer le prix de la tonne de carbone en 2020 de 35 dollars US à 24 dollars US (Piris Cabezas et Keohane 2008). Le rapport Eliasch (2008) conclut aussi à une exagération quant à la crainte exprimée par certains à propos d'une inondation possible du marché par les crédits REDD. Les négociants de crédits carbone (carbon traders), plusieurs pays d'Amérique latine et l'Indonésie sont en faveur d'une fongibilité sans réserve (voir Tableau 5.5).

Un certain nombre de propositions ont aussi pris en compte le risque d'inondation du marché et, par conséquent, de mise en danger de l'intégrité de l'environnement (Tableau 5.5). Au nombre de ces propositions figurent des propositions axées sur l'adoption d'objectifs géographiquement élargis et la régulation de la fongibilité des crédits REDD en créant un "double marché " (Ogonowski et al. 2007) et une nouvelle unité d'échanges spécifique à la REDD (Hare et Macey 2007).

Tableau 5.5. Propositions visant à rendre les crédits REDD fongibles sur les marchés du carbone

\begin{tabular}{|c|c|c|}
\hline Proposition & Description & Type \\
\hline $\begin{array}{l}\text { Fongibilité sans } \\
\text { réserve } \\
\text { (propositions des } \\
\text { pays incl. Belize, } \\
\text { Chili, Indonésie } \\
\text { et al.) }\end{array}$ & $\begin{array}{l}\text { Les crédits REDD sont vendus } \\
\text { en tant que paiements de } \\
\text { compensation aux pays de } \\
\text { l'Annexe } 1 \text {. La demande pour } \\
\text { REDD augmente en raison } \\
\text { de crédits REDD devenus } \\
\text { relativement bon marché. Il est } \\
\text { possible de plafonner le montant } \\
\text { des crédits admis dans le système }\end{array}$ & $\begin{array}{l}\text { Fongibilité sans réserve } \\
\text { des crédits REDD, } \\
\text { associée à des objectifs } \\
\text { de réduction d'émissions } \\
\text { géographiquement élargis } \\
\text { pour les pays de l'Annexe } \\
\mathrm{B} \text {, ayant pour résultat } \\
\text { une augmentation de la } \\
\text { demande de crédits }\end{array}$ \\
\hline $\begin{array}{l}\text { Double marché } \\
\text { (Center for Clean } \\
\text { Air Policy - CCAP) }\end{array}$ & $\begin{array}{l}\text { Crée un système d'échanges } \\
\text { propre à la REDD ; la demande est } \\
\text { provoquée par le transfert d'une } \\
\text { partie des engagements visés à } \\
\text { l'Annexe I au nouveau marché } \\
\text { (les montants dépendent des } \\
\text { objectifs globaux de l'Annexe I) }\end{array}$ & $\begin{array}{l}\text { Marché distinct mais } \\
\text { rattaché - transfert de } \\
\text { certains engagements } \\
\text { d'un marché actuel } \\
\text { vers un marché REDD. } \\
\text { La fongibilité peut } \\
\text { s'accentuer au fur et à } \\
\text { mesure des échéances du } \\
\text { marché REDD }\end{array}$ \\
\hline
\end{tabular}




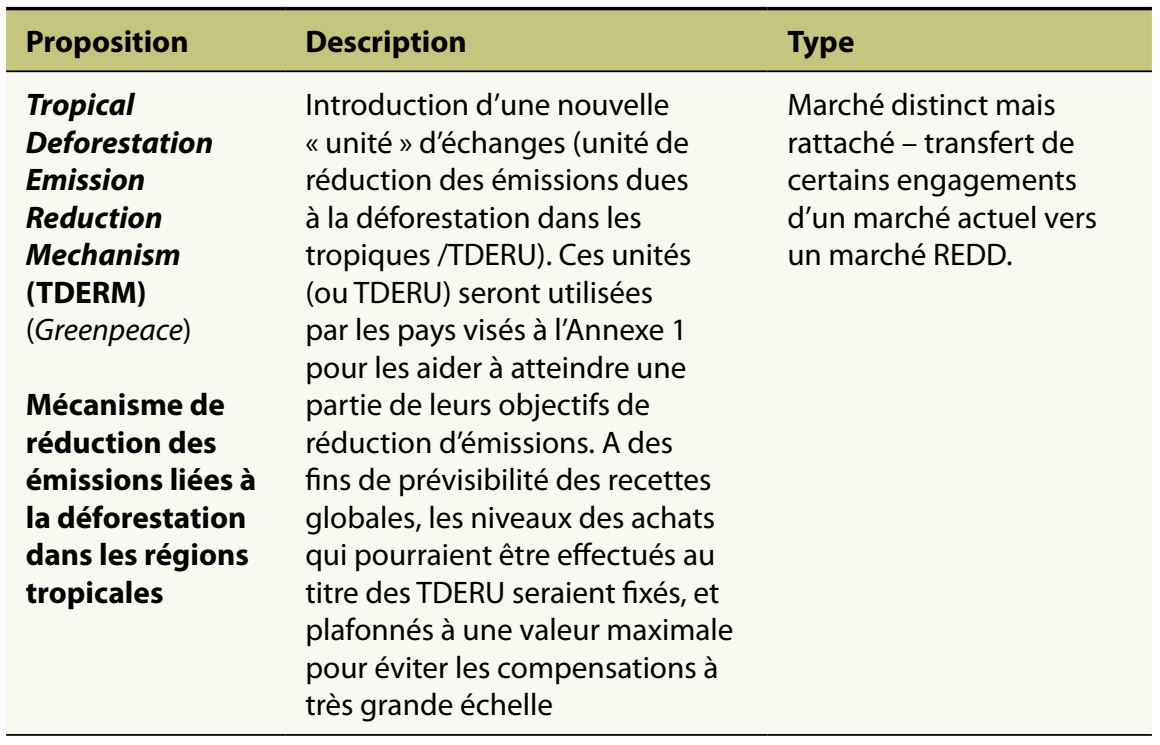

\subsubsection{Affectation du produit des enchères à un fonds REDD}

Une autre façon de collecter des fonds consiste à mettre aux enchères les permis d'émission provenant de projets de réductions d'émissions commercialisables, et d'affecter une partie du produit de la vente à un fonds REDD global. La loi Warner-Lieberman Bill (US), et le programme de l'UE Climate and Energy Package prévoient de verser une partie du produit de la vente aux enchères des droits d'émission au bénéfice de la REDD.

L'Union Européenne envisage d'affecter 5 pour cent du produit de la vente aux enchères au système de réduction des quotas d'émissions (ETS) de l'UE après 2012 au titre des efforts déployés à l'échelle planétaire pour lutter contre la déforestation. Cela permettrait de collecter des montants estimés de 2,0 à 2,7 milliards de dollars US par an jusqu'en 2020 (EC 2008). L'Allemagne vient récemment de s'engager à investir la totalité des recettes provenant de la vente aux enchères de ses droits d'émission UE dans des activités et des interventions stratégiques en faveur du climat aux niveaux national et international. Ces ventes aux enchères permettent de collecter des fonds en quantités substantielles. Le produit des ventes aux enchères représente ainsi - pour l'Allemagne toute seule - plus de 1 milliard d'Euros par an. Les recettes qui pourraient provenir de la mise aux enchères des droits d'émission liés aux émissions dues aux trafics aérien et maritime ont été estimées à 40 milliards de dollars US (Eliasch 2008). Le résultat d'une mise aux enchères de toutes les émissions des pays industrialisés pourrait atteindre au moins 100 milliards de dollars par an (Dutschke 2008). Ce dont on est moins certain cependant c'est de la proportion des montants de 
ces ventes qui serait effectivement reversée à la REDD, sachant que - du côté des bénéficiaires - d'autres secteurs et mécanismes, comme les transferts de technologies et les mesures d'adaptation (au changement climatique), seront en concurrence.

Certaines propositions présentées à la CCNUCC (CAN-International, Norvège) préconisent le recours à des ventes aux enchères de droits d'émission au niveau international (c.-à-d. les quantités attribuées ou assigned amount units $(\mathrm{AAU})$ du système de quotas d'émissions prévu dans le Protocole de Kyoto) en tant que moyen supplémentaire pour collecter des fonds au bénéfice de la REDD. ${ }^{1}$ En découplant la REDD des objectifs globaux des réductions d'émissions, ces propositions diminuent le risque d'inonder le marché. Reste la question critique de savoir comment garantir que le produit de ces ventes aux enchères sera effectivement affecté à des fins de réduction des émissions dues à la déforestation et à la dégradation des forêts (REDD).

\subsubsection{Attribution des recettes provenant d'autres redevances, amendes et taxes}

Une troisième proposition consiste à attribuer les recettes provenant des taxes et redevances à un mécanisme de financement de la REDD. Elles pourraient être liées à des marchés du carbone ou provenir d'autres marchés. Au nombre des options actuelles figurent les suivantes :

- Imposer une redevance sur le transfert des quantités attribuées (AAU) aux Parties au Protocole de Kyoto ou à d'autres secteurs/activités ;

- Porter le montant des amendes infligées aux pays n'ayant pas respecté leurs engagements sur un fonds d'affectation spécifique.

L'imposition d'une redevance sur un mécanisme de développement propre ou tout autre projet lié au carbone au niveau international peut être comparée au prélèvement de $2 \%$ effectué sur les transactions MDP pour appuyer le Fonds d'adaptation de la CCNUCC. Un système similaire pourrait aussi être appliqué au niveau national. La Chine, par exemple, a établi un système de taxation graduée des projets MDP pour réorienter le financement de grands projets industriels relevant du MDP vers des initiatives ayant plus d'impact sur le développement durable (Muller 2007). D'autres options incluent le prélèvement d'une taxe sur le transport aérien ou maritime international, qui pourrait générer des revenus à hauteur de 10 à 15 milliards de dollars US ou

\footnotetext{
1 Alors que les droits d'émission dans le marché du carbone de l'UE font déjà l'objet, en partie, de mise aux enchères, l'attribution d'unités AAU aux pays au titre du Protocole de Kyoto est gratuite. Selon CANInternational, la vente de quantités attribuées (AAU) au prix de 30 à 40 dollars US l'unité permettrait de recueillir 3,75 milliards de dollars US par an pour chaque poucent de quantités attribuées (AAU) vendues. La vente d'une fraction des quantités attribuées, par ex. 20 à $30 \%$, se solderait par un résultat total de 75 à 112,5 milliards de dollars US par an qui pourraient être mis à disposition des programmes d'adaptation (au changement climatique), de la REDD et du transfert des technologies (Scholz et Schmidt 2008).
} 
encore une taxe sur les transactions du marché monétaire international (taxe Tobin) qui pourrait rapporter à peu près le même montant (Eliasch 2008).

Ces mécanismes permettraient de recueillir des sommes substantielles pour financer la REDD mais comportent des failles si on les considère du point de vue de leur efficacité, de leur efficience (rentabilité) ou de l'équité - notamment en ce qui concerne une répartition équitable des revenus entre les pays et entre les secteurs d'activités. Les taxes et redevances influencent l'offre et la demande des activités de réduction d'émissions. Du côté de la demande, par exemple, imposer une redevance aux pays de l'Annexe I sur l'achat de quantités attribuées (AAU) pourrait avoir une incidence sur les affectations budgétaires en les détournant d'autres postes d'activité possibles.

En outre, certaines propositions pourraient être politiquement sensibles comme, par exemple, celle des amendes pour non-respect des engagements qui irait beaucoup plus loin que les mécanismes " peu contraignants " du Comité de contrôle du respect des dispositions du Protocole de Kyoto. De telles propositions seraient uniques en leur genre étant donné les systèmes d'application des dispositions traditionnellement peu contraignants des accords multilatéraux environnementaux.

\subsection{Combler les lacunes de financement}

Des financements à des niveaux sans précédent sont nécessaires pour exploiter pleinement le potentiel de la REDD et représentent l'un des défis principaux auxquels ce mécanisme est confronté. Le manque de financement pourrait être probablement plus prononcé : (i) pendant la période de démonstration du mécanisme international de la REDD avant 2012 ; et (ii) dans les pays dont les structures de gouvernance forestière sont fragiles et où, par conséquent, le contexte - pour les investissements - est à haut risque, comme c'est le cas de la plupart des régions de forêts tropicales.

L'APD peut apporter un soutien aux pays dont l'accès au marché est restreint, ce qui contribuerait à l'instauration d'une meilleure équité au niveau international. Il est aussi possible de pallier les manques de financement à travers des mécanismes appropriés comme en témoignent des mécanismes performants comme celui qui consiste à récompenser les actions anticipées (d'importance cruciale pour attirer les investissements privés anticipés et à haut risque) et à attribuer des crédits aux politiques et mesures (PM) de la REDD. Ce type de mesures pourrait atténuer toute incitation pernicieuse tendant à encourager les pays à accélérer leurs activités de déforestation avant 2012. Des mécanismes liés au marché comme ceux qui consistent notamment, à inclure les crédits REDD dans le marché du carbone, à mettre aux enchères les droits d'émission ou encore, à prélever des taxes et redevances sur les transactions de 
carbone, font partie des options les plus prometteuses pour pallier les manques de financement.

Quel que soit le scénario, il nous faut trouver des remèdes aux insuffisances des financements de source publique comme de source privée. Avant toute autre considération, il importe que le futur mécanisme qui sera mis en place pour la REDD soit ouvert à des approches flexibles et novatrices en matière de financement, afin de pouvoir s'adapter à l'évolution des besoins et des expériences dans les pays qui l'adopteront. 


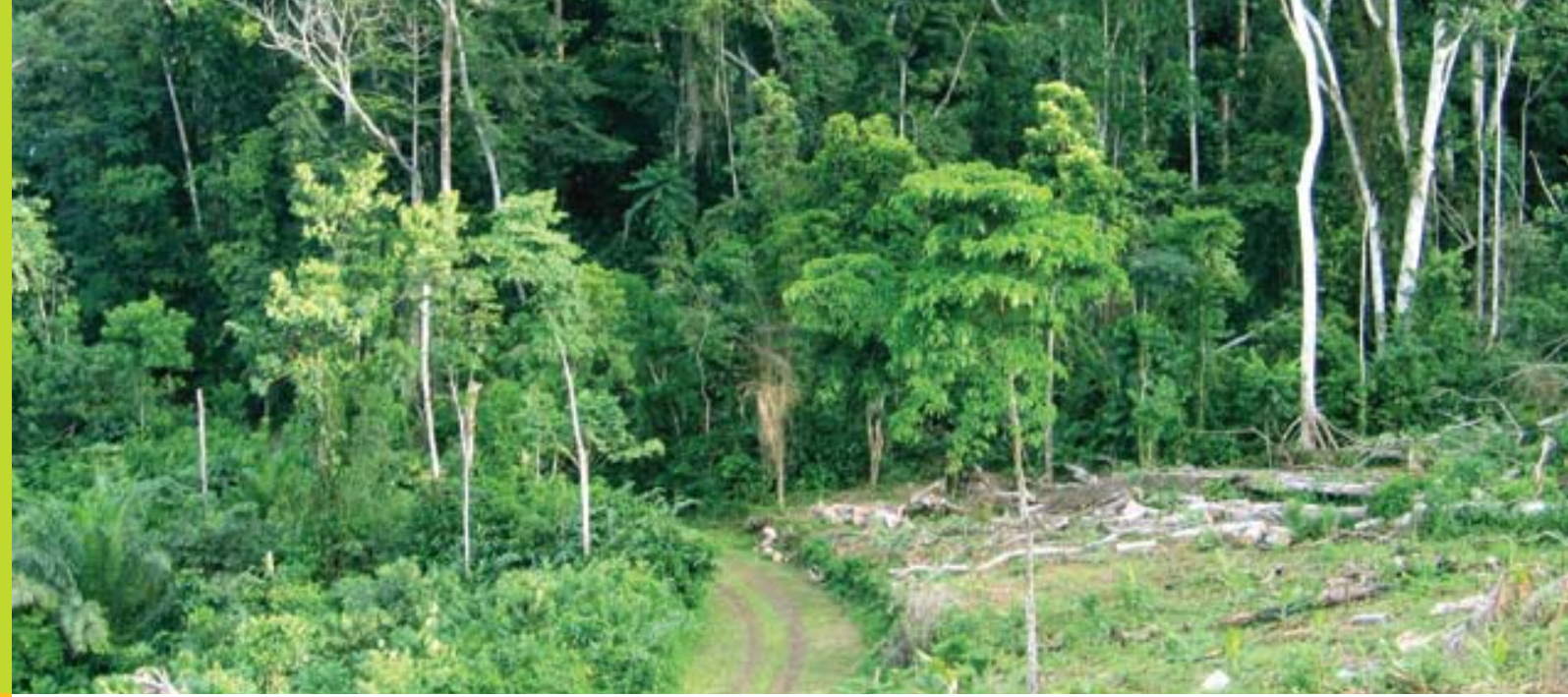

\title{
Chapitre 6
}

\section{Comment fixer les niveaux de référence des paiements REDD ?}

\author{
Arild Angelsen
}

\subsection{L'enjeu en cause}

L’un des éléments les plus critiques d'appréciation du nouveau régime envisagé à l'échelle mondiale pour " réduire les émissions dues à la déforestation et à la dégradation des forêts " (REDD) est de déterminer la façon dont les bases ou niveaux de référence seront fixés au niveau national. ${ }^{1}$ Les niveaux de référence ont de fortes incidences sur l'efficacité des actions en faveur de l'environnement, l'efficience des coûts (rentabilité) et la répartition des fonds de la REDD entre les différents pays. Il n'y a cependant pas de "formule » convenue sur la façon de les fixer. La plupart des soumissions qui sont présentées au titre de la REDD à la Convention cadre des Nations Unies sur les changements climatiques

\footnotetext{
Selon les cas, on utilise l'expression - «baseline » - "base de référence » dans le contexte du mécanisme pour un développement propre (MDP), ou - « reference line/level " - « niveau de référence " dans le contexte de la REDD. Cette distinction n'a pas été utilisée dans ce chapitre. Nous lui avons préféré la distinction établie entre Business as usual (BAU) (scenario de la poursuite de la actuelle tendance) et " crediting baselines" (bases de référence pour la comptabilisation) - expression que nous avons décrite succinctement dans ce chapitre - et utiliser l'expression "baseline " (base de référence) dans les deux cas, tandis que l'expression " reference line/level " (niveau de référence) sera utilisée au sens de " crediting baselines " (base de référence pour la comptabilisation).
} 
(CCNUCC) suggèrent d'utiliser les données historiques de déforestation mais de nombreux pays n'ont pas de données fiables à cet égard. De même, un soutien marqué s'est dégagé en faveur de l'inclusion des "circonstances nationales ", mais les répercussions sur le plan pratique restent encore à définir. Au vu des difficultés, certains pourraient « lever les bras au ciel de désespoir à l'idée de définir des niveaux de référence " (Pearce 2007 : 2). Il n'y a pas d'échappatoire possible cependant ; la question incontournable est de savoir quand (et comment) commencer à comptabiliser les réductions d'émissions.

Le débat est aussi noyé dans la terminologie, étant donné que les termes de "baseline " (base de référence) et de " reference line/level " (niveau de référence) se réfèrent au moins à trois choses différentes, illustrées à la Figure 6.1. En premier lieu, l'expression «baseline » (base de référence) peut se référer à la référence de base historique, c'est à dire au niveau de déforestation et de dégradation et aux émissions de $\mathrm{CO} 2$ ayant résulté sur un nombre x donné d'années écoulées. Deuxièmement, cette même expression "base de référence " peut se référer au scénario de la poursuite de la tendance actuelle (BAU) projeté : comment évolueront les émissions dues à la déforestation et à la dégradation en l'absence d'un mécanisme REDD ? Troisièmement, on peut aussi entendre par " baseline " (base de référence) la « crediting baseline " ou base de référence pour la comptabilisation (c.-à-d., un quota d'émissions, par exemple). Une base de référence BAU est l'étalon de mesure pour juger l'impact des mesures REDD qui ont été mises en œuvre (et garantir l'additionnalité), alors qu'une base de référence pour la comptabilisation est l'étalon de mesure pour récompenser le pays (ou le projet) si leur taux d'émission est inférieur

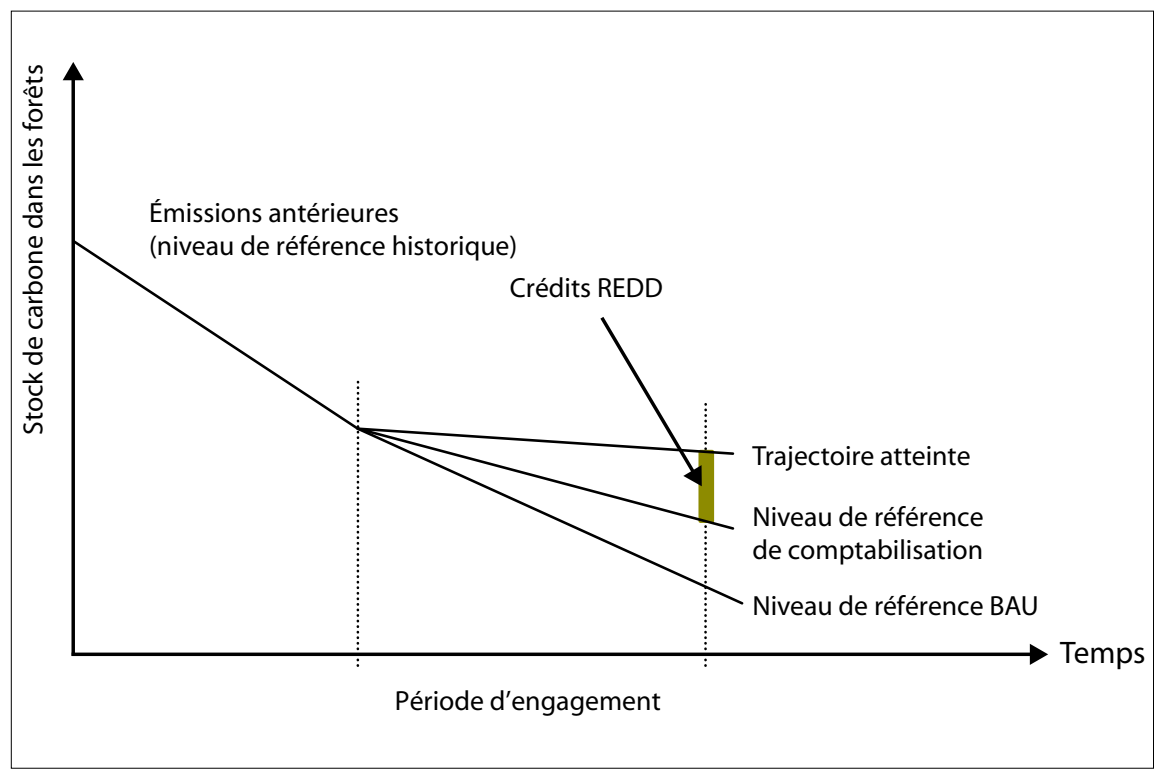

Figure 6.1. BAU (poursuite de la tendance actuelle) et crediting baselines (niveaux de référence pour la comptabilisation) 
au niveau de référence ou ne pas les récompenser, voire invoquer des soldes débiteurs si leur taux d'émission est supérieur à ce niveau (voir chapitre 8 sur les responsabilités).

Dans ce chapitre, une distinction a donc été établie entre le niveau de référence bistorique, le niveau de référence $\boldsymbol{B} \boldsymbol{A} \boldsymbol{U}$, et le niveau de référence pour $\boldsymbol{l} \boldsymbol{a}$ comptabilisation. Bien qu'aucune distinction entre des niveaux de références BAU et des références de base pour la comptabilisation n'ait été explicitement établie dans les soumissions, il est judicieux de juger des arguments en présence en replaçant les niveaux de référence dans deux contextes différents : (i) les bases de référence sont-elles de bons indices des futurs processus de déforestation et de dégradation des forêts (BAU) ? - question à laquelle pourraient, en principe, répondre les scientifiques sur la base de leurs connaissances actuelles des facteurs déterminants de déforestation et de dégradation; et (ii) constituent-elles des raisons acceptables pour fixer les niveaux de référence pour la comptabilisation? Cette deuxième question relève essentiellement du domaine politique et s'écarte très largement de considérations purement techniques.

\subsection{Le scénario de la poursuite de la tendance actuelle (BAU)}

\subsubsection{Déforestation historique au niveau national}

Le scénario BAU tente de répondre à une question entièrement hypothétique qui est la suivante : à quoi ressemblerait un processus de déforestation en l'absence de REDD ? Dans presque toutes les soumissions des Parties, ainsi que dans le Plan d'Action de Bali (COP 13), il est suggéré d'inclure dans les niveaux de référence les données nationales historiques de déforestation. La période de référence est généralement déterminée sur la base du taux moyen de déforestation des 10 années antérieures, et actualisée tous les trois ans, suivant une suggestion incluse dans une proposition plus ancienne présentée par Santilli et al. (2005). La période de référence exacte peut varier et une certaine flexibilité sera nécessaire au vu, par exemple, de l'existence ou non d'inventaires forestiers nationaux. Il y a lieu de noter que le choix de la période de référence historique peut avoir un impact considérable sur le scénario BAU, et les pays pourraient opter pour une stratégie basée sur des périodes de référence qui permettent d'optimiser les transferts liés à la REDD.

Dans quelle mesure les déforestations antérieures peuvent-elles nous permettre de prévoir les prochaines ? En premier lieu, les insuffisantes séries de données chronologiques dont disposent la plupart des pays en développement ne nous permettent pas d'en savoir autant que nous le souhaiterions. Deuxièmement, ce que nous savons laisse entendre que la déforestation passée n'est pas un indice précis de déforestation à venir (communication de la Nouvelle 


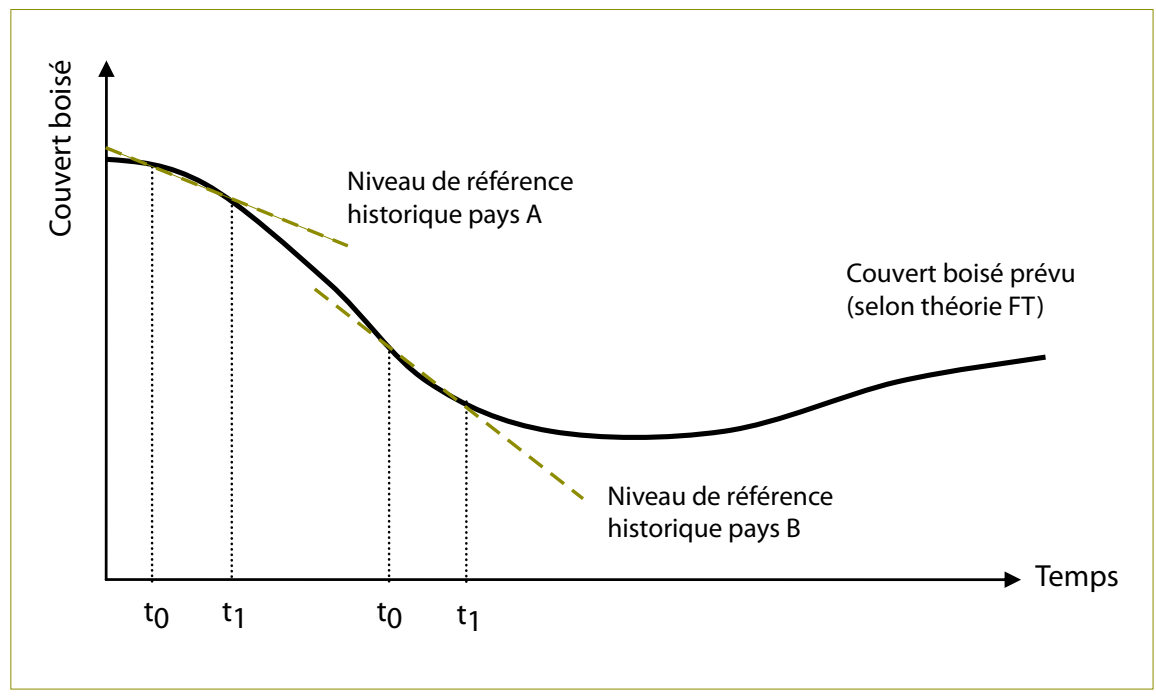

Figure 6.2. La transition des forêts (FT) et les niveaux de référence historiques

Zélande, avril 2008). A la différence des émissions de combustibles fossiles qui sont étroitement liées à une variable (produit intérieur brut, ou PIB), la déforestation a de multiples causes et peut beaucoup varier d'une année à l'autre. Elle peut aussi faire apparaître des tendances systématiques pendant des périodes plus longues ( 5 à 10 ans) qui diffèrent de schémas de déforestation antérieurs. Les fluctuations annuelles sont moins préoccupantes et peuvent être étudiées, par exemple, en utilisant des moyennes mobiles (par ex. les trois dernières années) ou des mécanismes prenant en compte la non-permanence et la responsabilisation (voir chapitre 8).

Un problème plus grave survient lorsque la déforestation historique sousévalue ou surévalue systématiquement le taux de déforestation dans un scénario BAU. Les variations de superficie forestière peuvent suivre un modèle - présenté dans la théorie de la "transition forestière " [Forest transition (FT) (Mather 1992 ; Angelsen 2007)] - dans lequel un pays donné, caractérisé au début du modèle par un fort pourcentage de terres boisées et un faible taux de déforestation, connaîtra une accélération du processus de déforestation, puis un ralentissement, une stabilisation du couvert forestier et, éventuellement une reprise. Ce modèle est illustré à la Figure 6.2. On peut avancer que des pays comme la Papouasie Nouvelle Guinée et la République démocratique du Congo qui se situent aux premiers stades de ce processus ont probablement un fort pourcentage de périmètres forestiers et des taux de déforestation peu élevés mais qui s'accélèrent. D’autres régions comme certaines parties du Brésil ou d'Indonésie qui se situent aux stades intermédiaires de ce même processus connaissent des taux élevés de déforestation qui devraient cependant baisser à mesure que les forêts deviennent plus clairsemées. Enfin, des pays ayant atteint les derniers stades de la transition comme la Chine et l'Inde (et 
un certain nombre de pays à hauts revenus) ont des périmètres forestiers en voie d'augmentation.

La transition des forêts n'est pas une « loi de la nature " et un certain nombre de facteurs en influencent le cours exact comme le contexte national, les forces économiques en présence et les stratégies adoptées par le gouvernement. Rendant compte, cependant, d'une tendance généralisée, la Figure 6.2 montre combien il est difficile de déterminer des niveaux de référence à partir de données ne portant que sur la déforestation historique. Une extrapolation des taux historiques sous-estime une future déforestation dans le contexte d'un scénario BAU pour les pays qui n'en sont qu'aux premiers stades de transition tandis qu'elle surestime ce même processus de déforestation dans les pays qui ont atteint des stades de transition plus avancés. ${ }^{2}$

\subsubsection{Circonstances nationales}

Proposé à la fois dans le Plan d'Action de Bali et dans un certain nombre de communications présentées par les pays, le deuxième élément à prendre en compte pour fixer les niveaux de référence est celui des « circonstances nationales ". Reste encore à déterminer quels sont les facteurs qui constituent des circonstances nationales légitimes (cf. la communication du Canada, mars 2008). L'une des propositions phare du débat (présentée, entre autres, par la Coalition for Rainforest Nations) est d'inclure un facteur d'ajustement au développement (DAF) dont une application pratique pourrait être que des niveaux de référence plus généreux seraient attribués aux pays dont le PIB par habitant est peu élevé, cette démarche pouvant être justifiée par plusieurs arguments. Au nombre de ces arguments figurent, notamment : (i) le fait que les pays les plus pauvres n'en sont probablement qu'aux premiers stades de la transition forestière et que, par conséquent, la déforestation (et la dégradation) devraient s'accélérer plutôt que ralentir dans un scénario BAU ; (ii) la capacité de mise en œuvre de la REDD pourrait être inversement proportionnelle au PIB par habitant, et des transferts plus importants seront nécessaires ; (iii) sur la base du principe de la CCNUCC des " responsabilités communes mais différenciées ", les conditions applicables dans le contexte de la REDD devraient être fixées à un niveau plus bas pour les pays les plus pauvres; et (iv) la REDD devrait constituer l'occasion d'un transfert de ressources aux pays les plus pauvres (bénéfices associés).

Il est aussi question d'étoffer la proposition sur la base d'une prise en compte des circonstances nationales à travers des modèles plus élaborés permettant

\footnotetext{
Noter que la transition des forêts décrit des variations de superficie forestière alors que ce qui nous intéresse concerne les variations des stocks de carbone forestier. Aux premiers stades de la transition, le carbone des forêts riches en carbone ne semble pas être utilisé alors que les densités de carbone (tonnes de carbone par ha) sont moindres aux stades plus avancés de la transition correspondant à une augmentation des périmètres boisés
} 
de faire des projections de déforestation sur la base de caractéristiques nationales spécifiques. Les modèles de régression déjà largement utilisés dans les études transversales sur la déforestation comportent un certain nombre de variables, dont certaines pourraient être incluses dans une formule permettant de déterminer les niveaux de référence (Angelsen et Kaimowitz 1999). Au nombre de ces facteurs (ou caractéristiques) figurent la densité et la croissance démographiques, la superficie forestière, la croissance économique, les prix des matières premières, les variables de la gouvernance, et la situation géographique (tropicale et régionale).

L'un des problèmes d'une approche modélisée tient au fait, par exemple, qu'elle est basée sur des valeurs projetées de la croissance démographique et économique et aussi sur des valeurs projetées des prix des matières premières. Motel et al. (2008) ont suggéré d'élargir cette approche en estimant l'impact des politiques gouvernementales après coup, autrement dit à la fin de la période de comptabilisation quand les informations requises sont disponibles. Les pays seraient alors récompensés pour avoir mené de front ces efforts et ces bonnes politiques ("Compensated Successful Effort" ou effort compensé réussi).

L'approche modélisée soulève plusieurs autres questions. D’abord, les séries de données chronologiques nécessaires sont, dans la plupart des pays, insuffisantes ou inexistantes. Deuxièmement, l'histoire de la déforestation modélisée nous montre que les modèles transversaux ne sont pas robustes, c'est-à-dire qu'il ne faut pas en attendre de réponse claire. Troisièmement, il n'est pas certain que les parties acceptent un chiffre de «boîte noire » en tant que niveau de référence.

\subsubsection{Déforestation historique à l'échelle mondiale}

Avancée par Achard et al. (2005) au titre d'une proposition du Joint Research Centre, une autre option vise à utiliser les taux de déforestation historiques mondiaux pour fixer les niveaux de référence individuels des pays. Les auteurs de la proposition suggèrent que les pays dont le taux de déforestation est inférieur à la moitié de la moyenne mondiale utilisent cette valeur comme base de référence nationale, tandis que les pays dont les taux de déforestation sont plus élevés utiliseraient un niveau de référence historique national. Différents scénarios peuvent aussi être envisagés sur la base de pondérations différentes pour la déforestation historique mondiale et la déforestation nationale (cf. Strassburg et al. 2008).

L'inclusion d'un facteur de déforestation mondiale dans la fixation des niveaux nationaux de référence se fonde sur deux hypothèses critiques. En premier lieu, on suppose que les différences de taux de déforestation reflètent des différences en matière de stratégies, et que les pays ne devraient pas être récompensés (punis) pour de bonnes (mauvaises) stratégies par l'attribution de niveaux de 
référence plus élevés (moins élevés). Un élément que l'on retrouve au cœur de maintes propositions est de "récompenser des actions anticipées". Si les stratégies constituent certes un facteur important pour expliquer les différences de taux de déforestation, les différences pour la plupart des pays dont les taux de déforestation sont peu élevés résultent en fait et avant tout d'autres facteurs, par exemple, du stade de transition forestière impulsé par le développement économique et la pénurie de forêts, plutôt que par l'adoption délibérée de politiques de conservation (Rudel et al. 2005).

Ensuite, la deuxième hypothèse de cette proposition est celle d'une certaine convergence des taux de déforestation dans le monde, à savoir que «à long terme, tous les pays en développement devraient connaître un taux de déboisement égal au taux moyen mondial de déforestation" (Eliasch 2008 : 136). Cette hypothèse prête à controverse et on manque de données empiriques pour la justifier. Bien au contraire, les éléments dont on dispose tendent à conforter la théorie de la transition forestière (Rudel et al. 2005 ; Chomitz et al. 2006), auquel cas il n'y aurait pas de convergence mondiale, mais plutôt des phases distinctes de variations du couvert boisé avec une lente augmentation de ce couvert au stade ultime de la transition.

\subsection{Niveaux de référence pour la comptabilisation}

Le niveau (ou base) de référence BAU est l'étalon de mesure pour évaluer l'impact des politiques et mesures REDD, alors que le niveau de référence pour la comptabilisation est l'étalon de mesure à partir duquel un pays (ou un projet) sera récompensé par l'attribution de crédits REDD ou d'autres formes de paiement. On pourrait, bien sûr, décider de fixer un niveau de référence de comptabilisation égal au niveau de référence BAU - ce qui est d'ailleurs l'hypothèse implicitement retenue. Mais il y a lieu de souligner la distinction importante - qui est d'ordre conceptuel - entre ces deux niveaux de référence, même si c'est s'aventurer sur le terrain de l'une des questions les plus litigieuses des négociations sur le climat: dans quelle mesure les pays en développement devraient-ils apporter - à un futur accord sur le climat - leur contribution à une réduction des émissions liées à la déforestation et à la dégradation des forêts (REDD) qui ne serait pas comptabilisée ?

Il existe trois raisons principales de ne pas superposer les niveaux de référence BAU et les niveaux de référence de comptabilisation. Tout d'abord, l'un des objectifs globaux du processus de la CCNUCC est de limiter les émissions mondiales de GES par rapport à un scénario BAU. Les responsabilités (reflétées dans leurs niveaux de référence de comptabilisation) attribuées aux pays en matière de réduction d'émissions doivent inévitablement s'ajouter pour atteindre 
l'objectif global de réduction d'émissions. La deuxième raison tient à la véritable crainte du déclenchement éventuel d'une situation où le fait d'avoir inclus des crédits REDD dans le marché réglementaire (des compensations) inonderait ce marché, autrement dit, ferait baisser le prix du carbone et supplanterait les autres activités d'atténuation (voir chapitres 3 et 5 pour plus de précisions). La fixation d'un niveau de référence plus bas pour la comptabilisation que pour le scénario BAU réduira le nombre de crédits commercialisés. En troisième lieu, il se pourrait qu'une partie des réductions d'émissions puissent être obtenues à travers des politiques et mesures appropriées (PM) et des approches financées par l'APD sans recours aux marchés.

Certains rapports d'orientation comme le rapport Eliasch (2008) suggèrent d'inclure la REDD dans un accord sur le climat sous forme de dispositions non contraignantes fondées sur des responsabilités limitées éliminant toute possibilité de perdre ("no-lose »), et de renforcer ensuite progressivement les engagements. La même idée est reprise dans la communication de Greenpeace (mars 2008), qui suggère d'augmenter les responsabilités des pays REDD en matière de réduction d'émissions à mesure que leurs circonstances économiques et l'agenda du climat mondial évoluent.

\subsubsection{Systèmes " sans perte »}

Une option pour faire en sorte que les pays participent à la REDD consiste, aux premiers stades de la mise en œuvre du mécanisme, à articuler les niveaux de référence de comptabilisation autour d'un système dans lequel aucun pays n'y perdrait, autrement dit un système "no-lose » ("sans perte»), similaire à celui $\mathrm{du}$ « sectoral no-lose targets» («cibles sectorielles sans perte») proposé par Ward et al. (2008). L'hypothèse « sans perte» - consistant à éliminer toute possibilité de perte - est sous-jacente à un grand nombre des négociations actuelles sur la REDD (bien qu'elle ait rarement été explicitée sous ce vocable).

Que signifie ce vocable «sans perte» sur le plan pratique? Al'évidence, un niveau de référence de comptabilisation qui serait fixé sans inclure de responsabilités (" niveau de référence et crédit ") et réputé égal à celui du scénario BAU constituerait pour les pays la garantie absolue qu'ils ne seront pas perdants en participant à la REDD. Il s'agit cependant d'une condition simplement suffisante et non nécessaire. Les pays REDD peuvent en effet continuer à tirer des bénéfices nets de leur participation à des systèmes dans lesquels les niveaux de référence de comptabilisation sont plus bas que ceux du scénario BAU, comme illustré dans l'encadré 6.1. La raison en est la suivante : pour les pays participant à la REDD, l'incitation à réduire la déforestation existera jusqu'au point où le coût marginal de réduction (c.-à-d. la courbe de l'offre nationale de REDD) sera égal aux compensations internationales, par exemple, au prix du marché des crédits REDD. A l'exception des réductions initiales moins 
onéreuses, ce prix sera supérieur aux coûts de réduction des émissions - ce qui génère ce que l'on pourrait appeler une rente REDD (zone $\mathrm{C}+\mathrm{D}$ dans la Figure). Grâce à cette rente, un pays peut obtenir un bénéfice net positif même si certaines des réductions initiales n’ont pas été compensées (payées), le niveau de référence comptabilisé pouvant être inférieur au niveau BAU. Les niveaux

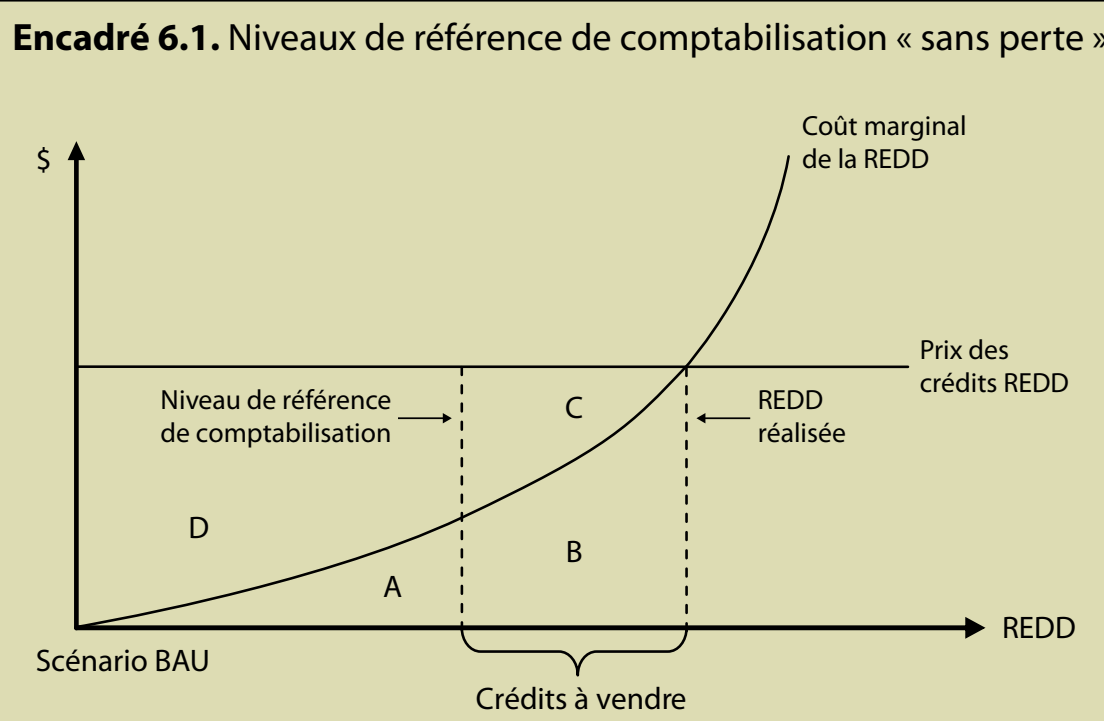

Les coûts marginaux de réduction en matière de déforestation et de dégradation commencent au niveau zéro dans le scénario BAU et augmentent à mesure que les réductions deviennent plus onéreuses (par exemple, quand sont exclues les utilisations de plus en plus rentables des terres agricoles). A un prix donné sur le marché international pour les crédits REDD, le pays réduira ses émissions jusqu'au point où les coûts marginaux équivaudront à ce prix (REDD réalisée). Le coût total de ces réductions est égal à la zone $A+B$. Un niveau de référence de comptabilisation est attribué et le pays perçoit un revenu correspondant à la vente des crédits REDD qui lui sont attribués au titre de ses réductions supérieures au niveau de référence de comptabilisation, autrement dit égales à la zone $B+C$. En définitive, les gains nets du pays équivaudront à $C-A$. Si le niveau de référence de comptabilisation est fixé au niveau de référence du scénario $B A U$, le gain du pays correspondra à la zone $C+D$, gain qui peut être qualifié de rente REDD.

Une question clé est de savoir quelle doit être le niveau de référence pour la comptabilisation afin qu'un pays puisse retirer un gain positif net de sa participation à la REDD. Si la courbe des coûts marginaux est linéaire, la réponse sera que le niveau de référence de comptabilisation devra être supérieur à une moitié de REDD réalisée. Mais des études empiriques ont montré que la courbe des coûts marginaux pour la REDD est convexe, comme illustré dans le schéma. Ainsi le niveau de référence de comptabilisation peut être déplacé plus loin à droite - autrement dit être inférieur à une moitié de REDD réalisée - et le pays continuera à retirer des bénéfices de sa participation à la REDD. 
de référence de comptabilisation "sans perte " peuvent donc implicitement comporter des réductions d'émissions qui n’ont pas été prises en compte.

\subsection{2 "Responsabilités communes mais différenciées »}

Principe reconnu de longue date par la CCNUCC et figurant aussi dans certaines soumissions concernant la REDD (par ex. Papouasie Nouvelle Guinée, août 2008), le principe des « responsabilités communes mais différenciées » est inscrit dans le Plan d'Action de Bali. Comme déjà indiqué, la proposition visant à prévoir un facteur d'ajustement pour tenir compte du développement (DAF), à travers lequel les niveaux de référence fixés pour la comptabilisation seraient plus favorables pour les pays les plus pauvres, peut être considérée comme une application pratique du principe selon lequel « certains pourcentages de déforestation seraient tolérés aux fins du développement socioéconomique d'un pays donné » (Alvardo et Wertz-Kanounnikoff, 2007 : 15).

Restent à mieux définir les conséquences possibles de l'application de ce principe sur le plan pratique. L'une d'entre elles pourrait être que chaque pays détient une part de responsabilité, c'est à dire que chacun d'entre eux doit venir à la table de négociations en étant prêt à des efforts qui ne seront pas nécessairement récompensés, tandis que les pays riches doivent faire encore plus à cet égard. Les conséquences réelles feront l'objet d'intenses négociations tard dans la nuit et ne seront pas examinées plus avant dans cet ouvrage.

\subsubsection{Quelques pistes de réflexion supplémentaires}

Etant donné les incertitudes liées à la détermination des niveaux de référence, une approche pratique a été proposée par Schlamadinger et al. (2005) sous le nom de "corridor approach " (approche corridor) avec un pourcentage croissant de réductions créditées. Par exemple, une réduction de la déforestation et de la dégradation qui passerait de $0,8 \%$ à $0,7 \%$ par an (ou l'équivalent en émissions de GES) génèrera des crédits carbone qui ne correspondront quà $20 \%$ des réductions d'émissions estimées alors que des réductions de $0,7 \%$ à $0,6 \%$ génèreront des crédits équivalant à $40 \%$ des réductions estimées, et ainsi de suite.

La déforestation et la dégradation qui surviennent après des processus naturels ou des catastrophes naturelles, par exemple après des ouragans, ne devraient pas être comptabilisées, ce qui serait conforme à la définition de la déforestation donnée dans la décision 11/CP7 de la CCNUCC, à savoir qu'il faut entendre par déforestation : "la conversion anthropique directe des terres forestières en terres non forestières ». Tout accord devrait en même temps prévoir des incitations visant à mieux gérer des risques « semi-naturels » comme les incendies. 


\subsection{Evaluation sur la base du critère des 3E}

Le critère des $3 \mathrm{E}$ applicable à l'évaluation de diverses propositions de modèles pour la REDD porte sur l'efficacité des crédits carbone, l'efficience des coûts, l'équité et les bénéfices y associés (voir chapitres 2 et 11). Les arguments concernant l'efficacité et l'efficience sont fondamentalement les mêmes et ont donc été réunis dans la même colonne du Tableau 6.1.

Tableau 6.1. Evaluation des propositions basées sur l'efficacité/l'efficience et l'équité

\begin{tabular}{lll}
\hline & Efficacité/efficience & $\begin{array}{l}\text { Equité (répartition } \\
\text { internationale) }\end{array}$ \\
\hline $\begin{array}{l}\text { Déforestation historique } \\
\text { au niveau national }\end{array}$ & $\begin{array}{l}\text { Les pays (riches en forêts) } \\
\text { où la déforestation est } \\
\text { peu importante } \\
\text { pourraient choisir de } \\
\text { dénoncer un accord }\end{array}$ & $\begin{array}{l}\text { Les pays riches comme } \\
\text { les pays pauvres en forêts } \\
\text { y perdent, les autres y } \\
\text { gagnent }\end{array}$ \\
$\begin{array}{lll}\text { Déforestation historique } \\
\text { au niveau mondial }\end{array}$ & $\begin{array}{l}\text { Risque « réductions } \\
\text { fictives » en provenance } \\
\text { de pays où le processus } \\
\text { de déforestation est peu } \\
\text { important }\end{array}$ & $\begin{array}{l}\text { Les pays à déforestation } \\
\text { importante y perdent, les } \\
\text { pays à déforestation peu } \\
\text { importante y gagnent }\end{array}$ \\
\hline $\begin{array}{l}\text { Circonstances nationales } \\
\text { (facteurs nationaux } \\
\text { spécifiques) }\end{array}$ & $\begin{array}{l}\text { Peut améliorer l'efficacité } \\
\text { si elles sont bien prises } \\
\text { en compte }\end{array}$ & $\begin{array}{l}\text { Dépend des facteurs pris } \\
\text { en compte }\end{array}$ \\
& $\begin{array}{l}\text { Risque de baisse } \\
\text { généralisée pour tous } \\
\text { les taux de réduction } \\
\text { d'émissions }\end{array}$ & $\begin{array}{l}\text { Certains pays (pauvres ?) } \\
\text { n'ont pas les moyens de } \\
\text { négocier des niveaux de } \\
\text { référence favorables }\end{array}$ \\
\hline $\begin{array}{l}\text { Flus attrayant pour les } \\
\text { pays pauvres qui sont } \\
\text { encouragés à participer } \\
\text { (crédits de référence } \\
\text { plus élevés pour les pays } \\
\text { pauvres) }\end{array}$ & $\begin{array}{l}\text { Bénéficie aux pays les plus } \\
\text { pauvres }\end{array}$ \\
\hline
\end{tabular}

Les niveaux de référence ont un effet sur l'efficacité à différents égards. S'ils sont trop contraignants un pays pourra estimer que les bénéfices nets ne sont ni assez élevés ni assez sûrs pour justifier sa participation. Par exemple, si un niveau de référence (pour la comptabilisation) supérieur au taux d'émissions historique n'est pas attribué aux pays qui se trouvent aux premiers stades de l'évolution de leurs paysages forestiers (taux de déboisement faible et densité forestière élevée) selon la théorie de la transition forestière (FT), il s'ensuivra que l'incitation de ces pays à participer à la REDD sera plus faible. En règle générale, il sera peut être nécessaire d'attribuer des niveaux de référence plus 
généreux pour obtenir une plus forte participation, mais cela accroîtra en même temps le risque de "réductions fictives" (voir ci-dessous).

Les effets de ces niveaux de référence (pour la comptabilisation) sur l'efficacité résultent aussi de l'impact des crédits carbone sur le marché mondial (en supposant acquise la permission d'introduire des crédits REDD sur ce marché). Nombre d'organisations non-gouvernementales de conservation de la nature (par ex. Leach, 2008) mettent en avant le risque éventuel "d'inondation du marché» par des crédits REDD bon marché. C'est, bien sûr, un scénario possible mais des moyens existent déjà pour le contrer : (i) diminuer l'offre de crédits REDD en abaissant les niveaux de référence ; (ii) augmenter la demande en introduisant des crédits REDD et en imposant, simultanément, des objectifs globaux plus contraignants en matière de réduction d'émission de GES (essentiellement, demandé par les pays de l'Annexe I) ; et (iii) instaurer un régime de fongibilité maîtrisée (limitée au début mais augmentant progressivement), par ex. en relevant le plafonnement imposé aux crédits REDD commercialisés (chapitre 5). La deuxième option est véritablement un argument majeur pour inclure la REDD dans un nouvel accord sur le climat: avec l'introduction d'une option d'atténuation peu onéreuse, les objectifs globaux peuvent devenir plus ambitieux.

Une crainte corrélée à ce qui précède tient à une éventualité que l'on a qualifiée risque de "réductions fictives ", c'est-à-dire que des crédits REDD ne correspondant pas à des efforts additionnels soient introduits sur le marché. Il y a lieu de noter que la création "de vent " n'est pas due à l'inclusion de réductions d'émissions à bas prix en tant que telles mais seulement à la mesure dans laquelle les niveaux de référence pour la comptabilisation des émissions ont été gonflés et fixés à une valeur supérieure à celle des niveaux de référence BAU (absence d'additionnalité). Le risque réel est qu'un grand nombre des critères utilisés pour fixer les niveaux de référence (par ex. les diverses circonstances nationales) pourraient aboutir à ces niveaux de référence gonflés, compromettant ainsi l'efficacité du système et sa crédibilité à long terme.

De même, l'utilisation de taux globaux de déforestation pour fixer les niveaux nationaux de référence implique un risque de "réductions fictives " à partir de pays dont le taux de déforestation est peu élevé. Nombreux sont ceux qui, parmi ces pays, sont susceptibles de se voir attribuer des niveaux de référence de comptabilisation supérieurs à leurs émissions dans un scénario BAU tandis que les acheteurs de crédits REDD s'attendent à payer des réductions réelles.

L'utilisation de critères différents pour fixer les niveaux de référence a des effets importants sur la distribution. De simples calculs d'approximation de distributions indiquent que la différence pour certains des plus grands pays forestiers tropicaux pourrait représenter plusieurs milliards de dollars US par 
an, ce qui apparaît aussi dans les scénarios simulant des méthodes de fixation différentes pour les niveaux de référence (par ex. Strassburg et al. 2008).

Les pays à faibles revenus richement dotés en forêts (aux premiers stades de la transition forestière) peuvent être les perdants si les niveaux de référence sont attribués uniquement sur la base de taux nationaux de déforestation. Sur la base de taux mondiaux de déforestation historique, ce sont les pays à taux de déforestation élevé (aux stades intermédiaires de la transition forestière) qui pourraient être les perdants. L'inclusion d'un facteur d'ajustement pour tenir compte du développement (DAF) bénéficierait aux pays les plus pauvres et ferait du mécanisme REDD un mécanisme davantage en faveur des pauvres (axé sur les initiatives en faveur des pauvres).

\subsection{Conclusion}

Les niveaux de référence font partie des éléments constitutifs critiques du nouveau régime de réduction des émissions envisagé pour réduire les émissions liées à la déforestation et à la dégradation des forêts (REDD), aussi bien du point de vue de leur efficacité et de leur répartition dans le monde que de celui de leur équité. Les conflits d'intérêt abondent et sont évidents entre (groupes de) pays, et (en partie pour cette raison) la question est politiquement sensible. Presque toutes les soumissions (propositions) utilisent la déforestation historique comme point de départ et la plupart laissent aussi entendre que les " circonstances nationales » et « la récompense des actions anticipées " soient prises en compte. Il reste à mettre ces principes en application. Une façon d'avancer dans ce débat consiste à établir une meilleure distinction entre les deux types de références étudiés dans ce chapitre: (i) les prévisions relatives à la déforestation et à la dégradation dans un scénario de la poursuite de la situation actuelle (BAU) ; et (ii) les niveaux de référence (pour la comptabilisation) qui seront établis sur la base du scénario BAU et d'une série de considérations politiques ainsi que du poids du pays dans les négociations.

Il ne fait pas de doute que les négociateurs seront confrontés à un dilemme clé. D’un côté, le choix de niveaux de référence généreux, fixés sur la base d'études spécifiques par pays pour prendre en compte les circonstances nationales, pourrait entraîner la création de " réductions fictives ", susceptible de compromettre l'intégrité de l'environnement (réductions globales des émissions de GES) et la crédibilité du mécanisme REDD. De l'autre côté, un accord qui fixerait des niveaux de référence de comptabilisation peu ambitieux pourrait être considéré comme inacceptable par les pays participant à la REDD. Bref, il s'agira d'un délicat exercice d'équilibre entre le risque de " réductions fictives » et la participation des pays à la REDD. 



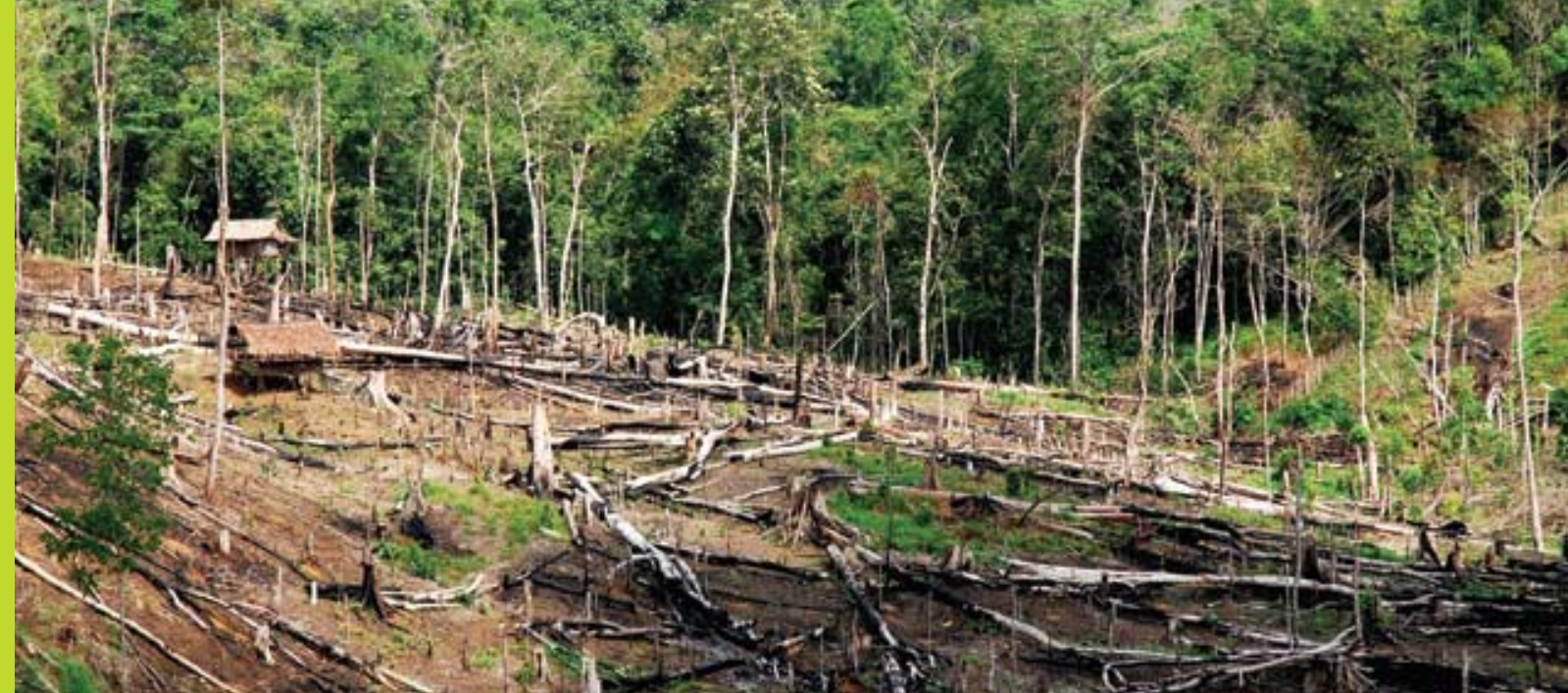

\title{
Chapitie 7
}

\section{Comment remédier aux fuites?}

\author{
Sven Wunder
}

\subsection{Introduction}

Imaginez que vous vivez au bord d'un lac de montagne. Récemment, la fonte des glaces causée par le réchauffement climatique a sérieusement inondé vos terres à plusieurs reprises. Vous décidez donc de construire une digue pour protéger les terres les plus basses et les plus inondables en contrebas. Cependant, comme le lac est de petites dimensions, vous allez de ce fait entraîner une nouvelle hausse du niveau de ses eaux, ce qui provoquera l'inondation de zones antérieurement épargnées. Si votre objectif primordial était de protéger et de rendre moins inondables vos terres en bordure de lac, il vous faudra déduire les " fuites " des gains projetés résultant de votre projet de construction d'une digue, c'est-à-dire chiffrer les pertes dues au déplacement des zones inondables ailleurs dans l'espace.

En principe, les fuites de carbone ont des effets similaires, au sens où ils se produisent à un autre endroit que celui de la fuite. Alors que les 37 pays développés visés à l'Annexe I du Protocole de Kyoto sont convenus de plafonner leurs émissions industrielles, les importations en hausse des pays non visés à l'Annexe I pourraient être à l'origine de "fuites " d'émissions. 
Les réductions nettes d'émissions de gaz à effet de serre (GES) dans une zone donnée subissent les effets d'émissions attribuables à des projets situés en dehors des zones ciblées par des mesures d'atténuation. Des fuites peuvent survenir quand l'échelle spatiale d'intervention est inférieure à l'échelle globale du problème ciblé. L'atténuation des émissions de $\mathrm{CO}_{2}$ est un objectif global, ce qui fait que des fuites peuvent survenir à diverses échelles, de l'exploitation agricole aux niveaux local/régional, national ou international - et dans de nombreux secteurs, y compris dans le cas de projets d'atténuation des effets du changement climatique dans le secteur forestier et celui de l'énergie. Les fuites de carbone sont essentiellement un processus économique même s'il y a d'autres interférences à caractère anthropique et biophysique. Contrairement à l'exemple donné au début de ce chapitre - celui de la digue - où les pressions (les zones inondables) sont exclusivement exportées, une fuite peut aussi avoir l'effet inverse, c.-à-d. d'importer d'autres réductions d'émissions de zones situées en dehors de la zone d'atténuation ciblée (dénommée « fuite inversée »).

Prenons pour exemple un projet de réduction d'émissions dues à la déforestation et à la dégradation des forêts (REDD) associé à un programme PSE (paiements pour services environnementaux) pour exploitations agricoles au titre duquel un propriétaire foncier pourrait être récompensé pour n'avoir pas déboisé sa parcelle forestière $(\mathrm{A})$ - inscrite au programme PSE - pendant une durée de cinq ans. Si le propriétaire a cependant déplacé tous ses projets de déboisement de la parcelle $(\mathrm{A})$ vers une autre parcelle, à savoir la parcelle $(\mathrm{B})$ - non inscrite au programme PSE - la mesure d'atténuation serait entièrement compensée par la fuite ou le "déplacement d'émissions " (pour reprendre l'expression utilisée dans le Plan d'Action de Bali à la treizième Session de la Conférence des Parties -COP 13). Si le propriétaire utilise en outre tous les fonds PSE pour acheter des tronçonneuses afin de défricher davantage pour ensuite laisser le bétail paître sur ces terres, les fuites à moyen terme pourraient représenter plus de 100 pour cent de l'atténuation - ce qui implique que la fuite a aussi une dimension temporelle - selon la rapidité des processus biophysiques et économiques. A contrario, si le propriétaire investit les fonds PSE dans l'écotourisme ou l'agroforesterie et cesse tout défrichement, la fuite serait inversée et importerait des gains d'atténuation provenant de sites autres que la seule parcelle-cible (A).

\subsection{Dimensions de la fuite}

\subsubsection{Voies de transmission des fuites}

Certains analystes établissent une distinction entre une fuite primaire (déplacement d'activités) causée par des parties prenantes à la REDD et une fuite secondaire (marché ou équilibre partiel/général) causée par des tiers, par ex. en réponse à des variations de prix (Aukland et al. 2003). Le Tableau 7.1 décrit les 
grandes différences existant entre les trois types de projets d'atténuation quant aux probabilités de fuites. S’agissant des activités au titre de la REDD (les deux dernières colonnes), les zones de conservation protégées sont distinguées des projets de gestion durable des forêts (GDF).

Tableau 7.1. Impact des mesures d'atténuation sur la probabilité de fuites et voies de transmission

\begin{tabular}{|c|c|c|c|}
\hline Types de projets & \multirow{2}{*}{$\begin{array}{l}\text { Boisement et } \\
\text { reboisement }(B / R)\end{array}$} & \multirow{2}{*}{$\begin{array}{l}\text { REDD - Zones de } \\
\text { conservation }\end{array}$} & \multirow{2}{*}{$\begin{array}{l}\text { REDD - Gestion } \\
\text { durable des forêts }\end{array}$} \\
\hline $\begin{array}{l}\text { Voies de } \\
\text { transmission des } \\
\text { fuites }\end{array}$ & & & \\
\hline $\begin{array}{l}\text { A. Marchés } \\
\text { fonciers }\end{array}$ & $\begin{array}{l}\text { Remplacement de } \\
\text { cultures/élevage } \\
\text { par plantations }\end{array}$ & $\begin{array}{l}\text { Freine la conversion } \\
\text { planifiée en terres } \\
\text { agricoles }\end{array}$ & $\begin{array}{l}\text { Freine la conversion } \\
\text { planifiée en terres } \\
\text { agricoles }\end{array}$ \\
\hline $\begin{array}{l}\text { B. Marchés du } \\
\text { travail }\end{array}$ & $\begin{array}{l}\text { Utilisation de main } \\
\text { d'œuvre au début ; } \\
\text { variable ensuite }\end{array}$ & $\begin{array}{l}\text { Baisse de l'emploi } \\
\text { peut entraîner une } \\
\text { migration externe }\end{array}$ & $\begin{array}{l}\text { Economie ou } \\
\text { déplacement de } \\
\text { main-d'œuvre? }\end{array}$ \\
\hline $\begin{array}{l}\text { C. Marchés des } \\
\text { capitaux }\end{array}$ & $\begin{array}{l}\text { Rentabilité peut } \\
\text { attirer capitaux }\end{array}$ & $\begin{array}{l}\text { Exportation due } \\
\text { à une rentabilité } \\
\text { moindre }\end{array}$ & $\begin{array}{l}\text { Impact sur la } \\
\text { rentabilité ? } \\
\text { controversé }\end{array}$ \\
\hline $\begin{array}{l}\text { D. Innovations } \\
\text { technologiques }\end{array}$ & Variable & $\begin{array}{l}\text { Aucun (à moins } \\
\text { d'être combinés à } \\
\text { l'écotourisme, aux } \\
\text { produits forestiers } \\
\text { non ligneux) }\end{array}$ & $\begin{array}{l}\text { Limitation des effets } \\
\text { de l'abattage, etc. }\end{array}$ \\
\hline $\begin{array}{l}\text { E. Marchés de la } \\
\text { production }\end{array}$ & $\begin{array}{l}\text { Produits des forêts } \\
\text { plantées (à moyen } \\
\text { terme) diminuent } \\
\text { les pressions sur } \\
\text { l'extraction }\end{array}$ & $\begin{array}{l}\text { Pas d'offre de } \\
\text { produits agricoles } \\
\text { ou de bois } \\
\text { provenant des } \\
\text { zones protégées } \\
\end{array}$ & $\begin{array}{l}\text { Moins de bois (à court } \\
\text { ou moyen terme) }\end{array}$ \\
\hline $\begin{array}{l}\text { F. Production de } \\
\text { revenus }\end{array}$ & Variable & Variable & Variable \\
\hline $\begin{array}{l}\text { G. Conditions } \\
\text { écologiques }\end{array}$ & $\begin{array}{l}\text { Plantations font } \\
\text { augmenter ou } \\
\text { diminuer l'intégrité } \\
\text { écologique } \\
\text { (ennemis des } \\
\text { cultures, vent, } \\
\text { biodiversité, etc.) }\end{array}$ & $\begin{array}{l}\text { Augmentation } \\
\text { de l'intégrité } \\
\text { du paysage et } \\
\text { adaptation, « effets } \\
\text { marginaux » évités }\end{array}$ & $\begin{array}{l}\text { Augmentation de } \\
\text { l'intégrité du paysage } \\
\text { et adaptation, « effets } \\
\text { marginaux » évités }\end{array}$ \\
\hline
\end{tabular}

Note : Les cellules gris claires indiquent une fuite (diminution hors site avec effet d'atténuation net), les cellules gris foncé indiquent une fuite inversée (augmentation hors site avec effet d'atténuation net), et les cellules blanches indiquent des impacts ambigus/ dépendant du contexte. 
Les déplacements de la demande foncière, que ce soit à travers des marchés fonciers concurrents ou d'autres mécanismes de substitution spatiaux, sont la force causale dominante des fuites pour la REDD (conservation et GDF) : comme la déforestation est essentiellement due à la conversion des terres forestières en terres agricoles, la fermeture de la frontière agricole créera une situation de pénurie foncière, à moins que l'utilisation de pratiques appropriées, par ex. la diminution du temps de mise en jachère ou l'enrichissement des pâturages, permette une agriculture plus intensive. En matière foncière, les pénuries provoquées sont plus prononcées pour la REDD que pour les activités de boisement et de reboisement $(B / R)$ qui sont souvent effectuées sur des terres dégradées de faible valeur économique.

S'agissant des activités REDD, les zones de conservation semblent nécessiter moins de main d'œuvre par hectare que la plupart des autres terres réaffectées à d'autres utilisations, ce qui pourrait entraîner des migrations externes et des effets de déplacement des émissions de GES ; s'agissant des projets de boisement/reboisement $(\mathrm{B} / \mathrm{R})$ et de gestion durable des forêts (GDF), les impacts sur l'emploi dépendent de la durée et du contexte. Les marchés de capitaux font partie des forces les plus fongibles : comme dans l'exemple de la digue où l'eau s'écoule doucement vers les recoins les plus éloignés, les capitaux financiers s'écoulent en règle générale tranquillement vers des options à forte rentabilité. Il leur sera donc possible de réagir à une situation de moindre rentabilité causée par l'application de mesures d'atténuation en finançant des options plus rentables ailleurs. Les activités de boisement/reboisement (B/R) et de GDF peuvent cependant parfois présenter des opportunités d'investissement intéressantes ; dans ce cas, elles importeront des capitaux et seront à l'origine de fuites inversées.

Les innovations technologiques en GDF (par ex. limitation de l'impact de l'abattage) peuvent réduire la dégradation de la forêt dans les zones avoisinantes, mais l'effet de propagation des technologies est habituellement négligeable pour la conservation comme pour le boisement/reboisement. S'agissant des marchés de production, les activités de boisement/reboisement augmenteront l'offre future de bois (tout en réduisant probablement les productions agricole et animale). Au contraire, la conservation liée à la REDD réduira l'offre (à court terme) de produits ligneux (bois) et agricoles, augmentera les prix des produits de base et devrait ainsi stimuler la production dans d'autres secteurs/ zones. Il y a lieu de noter qu'une diminution de la déforestation dans les zones protégées pourrait induire une dégradation de la forêt sur d'autres sites à travers les marchés du bois. La gestion durable des forêts (GDF) limite aussi l'expansion des cultures et de l'élevage mais assure une exploitation du bois et un approvisionnement durables dans le temps.

Il est difficile de démêler aussi bien les effets complexes entraînés par les projets d'atténuation sur les revenus ou les relations de production en aval 
et en amont que de déterminer à priori d'autres changements de trajectoires de développement dus à ce même type de projets. Tous ces facteurs peuvent cependant avoir un effet important sur les fuites et doivent donc être vérifiés. Enfin, la REDD peut contribuer au maintien de paysages écologiquement sains, y compris en les rendant plus adaptables au changement climatique, en évitant les " effets marginaux » de la dégradation des forêts et, par conséquent, en réduisant les émissions de GES hors site. Les fuites inversées liées à la REDD devraient être plus importantes que celles qui relèvent des projets de boisement/reboisement, dominés par les monocultures.

\subsubsection{Taille et importance}

Le Tableau 7.1 a montré que les forces de fuite (cellules grises) pouvaient être potentiellement plus significatives dans le cas de la REDD que dans celui des activités de boisement/reboisement (B/R), essentiellement parce que la REDD tend sans ambiguïté à limiter un développement local basé sur la conversion des terres forestières pour le développement. La gestion durable des forêts (GDF) entraîne probablement moins de possibilités de fuites que la conservation de zones protégées, mais un taux d'adoption décevant dans les régions tropicales indique aussi qu'elle est plus difficile à mettre en œuvre. Y a-t-il davantage de fuites liées à la REDD qu'à des projets $\mathrm{B} / \mathrm{R}$ ou à des projets basés sur l'énergie? Les fuites liées aux projets axés sur l'énergie ont été estimées dans le 2e Rapport d'évaluation du Groupe intergouvernemental d'experts sur l'évolution du climat (GIEC) dans une large fourchette de 0-70\%, mais ont été réduites plus tard à 5-20\%. Il y a peu de raisons de penser que les projets $\mathrm{B} / \mathrm{R}$ donnent lieu à des fuites plus élevées (Chomitz 2000). De récentes études de cas confirment cette opinion, par ex. une légère fuite inversée a été constatée dans le projet communautaire de plantation d'arbres de Scolel Té, déjà vieux de 10 ans, au Chiapas, Mexique (de Jong et al. 2007). Sathaye et Andrasko (2007 : 966) concluent que « les analyses à ce jour ont montré que les probabilités de fuite sont bien plus élevées (0-92\%) dans les cas de déforestation évitée et semblent augmenter à mesure que s'étend la région géographique étudiée ». Wu (2000) a calculé des effets dus aux fuites dans le Programme de conservation et de mise en réserve des terres aux Etats-Unis) atteignant environ $20 \%$. Un seul projet REDD en région tropicale a fait l'objet d'une étude approfondie : le projet Noel Kempff en Bolivie (Encadré 7.1). Les difficultés inhérentes à la fixation de niveaux de référence pour la REDD, avec deux ordres de grandeur de variations pour trois projections de modèles possibles, illustrent le plus grand problème actuel : le petit nombre de projets REDD réellement appliqués sur le terrain et les modèles préliminaires de quantification laissent un immense champ libre à la spéculation. Nous ne connaissons donc pas réellement l'ampleur des fuites liées à la REDD, ni leur importance par rapport à celles d'autres secteurs (Schwarze et al. 2002). 


\section{Encadré 7.1. Le projet Noel Kempff : Atténuation des émissions de $\mathrm{CO}_{2}$ en réduisant l'abattage et la déforestation}

En 1997, trois compagnies d'électricité intéressées par le marché volontaire du carbone ont uni leurs forces à celles de l'organisation The Nature Conservancy et investi 9,5 millions de dollars US dans les 634,000 ha de l'extension du Parc National Noel Kempff à l'est de la Bolivie. Ces millions ont servi principalement à racheter des concessions d'exploitation de bois et de grandes propriétés foncières, et à lancer des projets de conservation et de développement intégrés dans trois communautés locales. D'abord axé sur l'abattage évité (dégradation des forêts), le centre d'intérêt s'est déplacé vers la conversion évitée des terres forestières en terres agricoles (déforestation). La prévention des fuites primaires a constitué l'élément clé du modèle contractuel, en empêchant en particulier les exploitants forestiers des concessions rachetées de se réinstaller tout simplement ailleurs en Bolivie. Les fuites pouvant correspondre à la cessation des activités d'abattage ont été soigneusement examinées et établies pour une gamme de 2 à $42 \%$, les taux de décomposition du bois et les élasticités de la demande de bois constituant les facteurs les plus influents. Le processus de déforestation s'est en fait accru initialement dans les communautés locales - ce que l'on a espéré constituer un phénomène transitoire - en raison de la création de nouveaux systèmes d'utilisation des terres. La procédure de fixation de niveaux de référence pour la déforestation et l'abattage s'est révélée délicate à mettre en œuvre. Trois modèles simulant des niveaux de référence pour les émissions de $\mathrm{CO}_{2}$ sur une période de plus de 20 ans ont abouti à des résultats extrêmement différents : $F A C=11.54 \mathrm{TgC}$, GEOMOD $=1.05 \mathrm{TgC}$, et $\mathrm{LUCS}=0.18 \mathrm{TgC}$. S'agissant des fuites liées à l'exploitation du bois, un modèle dynamique optimisé a été utilisé. Pour ce qui est de la modélisation spatiale des fuites liées à la déforestation, GEOMOD est le modèle qui conviendrait le mieux, mais la déforestation prévue dans le cadre du projet à court terme n'avait pas encore été rendue effective, et l'éloignement semble apparemment continuer à limiter le risque d'une colonisation accrue des terres par des squatters venus des zones tampon.

Sources : Winrock (2002), Sohngen et Brown (2004), Brown et al. (2007)

\subsubsection{Facteurs déterminants des fuites}

Peu de projets REDD sont mis en œuvre actuellement et il est donc prématuré de demander des estimations crédibles de fuites ou des recettes de modèles résistants aux fuites. S'il est utile de faire des simulations chiffrées, les écarts de prévisions restent cependant beaucoup trop larges pour être acceptables.

A l'échelle des pays, les fuites peuvent avoir un effet significatif sur les projets sous-nationaux qui relèvent de la REDD. Si une nation perd $1 \%$ de son couvert boisé par an, cela signifie que $99 \%$ de son couvert n'est donc actuellement pas menacé. Les partisans de l'efficience des coûts pour la REDD, notamment le Rapport Stern, supposent que ce $1 \%$ peut être identifié avec exactitude, ce qui, à l'évidence, est une position irréaliste. Certes, la déforestation est très concentrée dans les zones d'avancée des frontières agricoles, par ex. dans l'Arc de déforestation brésilien. Les modélisations spatiales pour le Mexique 
permettent maintenant de faire des projections de deux tiers de déforestation, en utilisant des variables telles que la proximité des routes et des marchés, la qualité du sol, les pentes, la croissance de la population, etc. Cependant, dans les zones où prévaut le défrichement progressif d'îlots forestiers dans les paysages agricoles, il sera bien plus difficile de faire des prévisions spatiales en matière de déforestation et de démêler tous les aspects des fuites que la situation sur le terrain rend encore plus complexes. Dans ce contexte, les erreurs de prévisions spatiales et le caractère plus fongible dans l'espace des facteurs économiques contraignants, sous-entendent qu'il sera nécessaire de protéger simultanément d'autres réserves forestières, outre celles qui sont initialement menacées (les îlots forestiers). Ces menaces de fuites qui s'étendent à de multiples sites peuvent sensiblement accroître les coûts liés à la REDD.

Le bon sens peut toutefois aider dans l'exercice consistant à faire une évaluation préliminaire des fuites dans différent sites et scénarios (voir Figure 7.1). Tout d'abord, si la main d'œuvre et le capital sont très mobiles, les activités et émissions déplacées par la REDD s'écouleront facilement ailleurs (a). Si des périmètres forestiers adjacents - dotés de sols de bonne qualité mais mal protégés par une réglementation insuffisante ou caractérisés par des prix fonciers bon marché - sont disponibles, il existe une plus forte probabilité que des fuites se produisent dans ces périmètres plutôt que dans d'autres qui seraient plus éloignés, bien protégés, plus onéreux et/ou moins susceptibles d'être convertis à d'autres utilisations (b). Si la demande de produits soumis à des restrictions au titre de la REDD (bois, cultures, production animale, etc.) est inélastique par rapport au prix - autrement dit que la réduction de l'offre (suscitée par la REDD) n'aura pas pour résultat une forte diminution de la demande - il existe alors une plus forte probabilité de fuite de l'activité (c). Des technologies de production flexibles peuvent aider à compenser au niveau local (d) la pénurie de terres causée par les zones de conservation mises de côté et protégées dans le cadre de la REDD, par ex. en transformant l'élevage extensif de bétail amazonien en élevage intensif grâce à une rénovation des pâturages ou au quasi-abandon des cultures sur brûlis grâce à une meilleure fertilisation. Inversement, si la production mécanisée de soja dépend de technologies basées sur des coefficients d'intrants fixes, il en résultera l'impossibilité d'appliquer des mesures d'adaptation au niveau local pour économiser des terres et, par conséquent, une plus grande probabilité de fuites. 


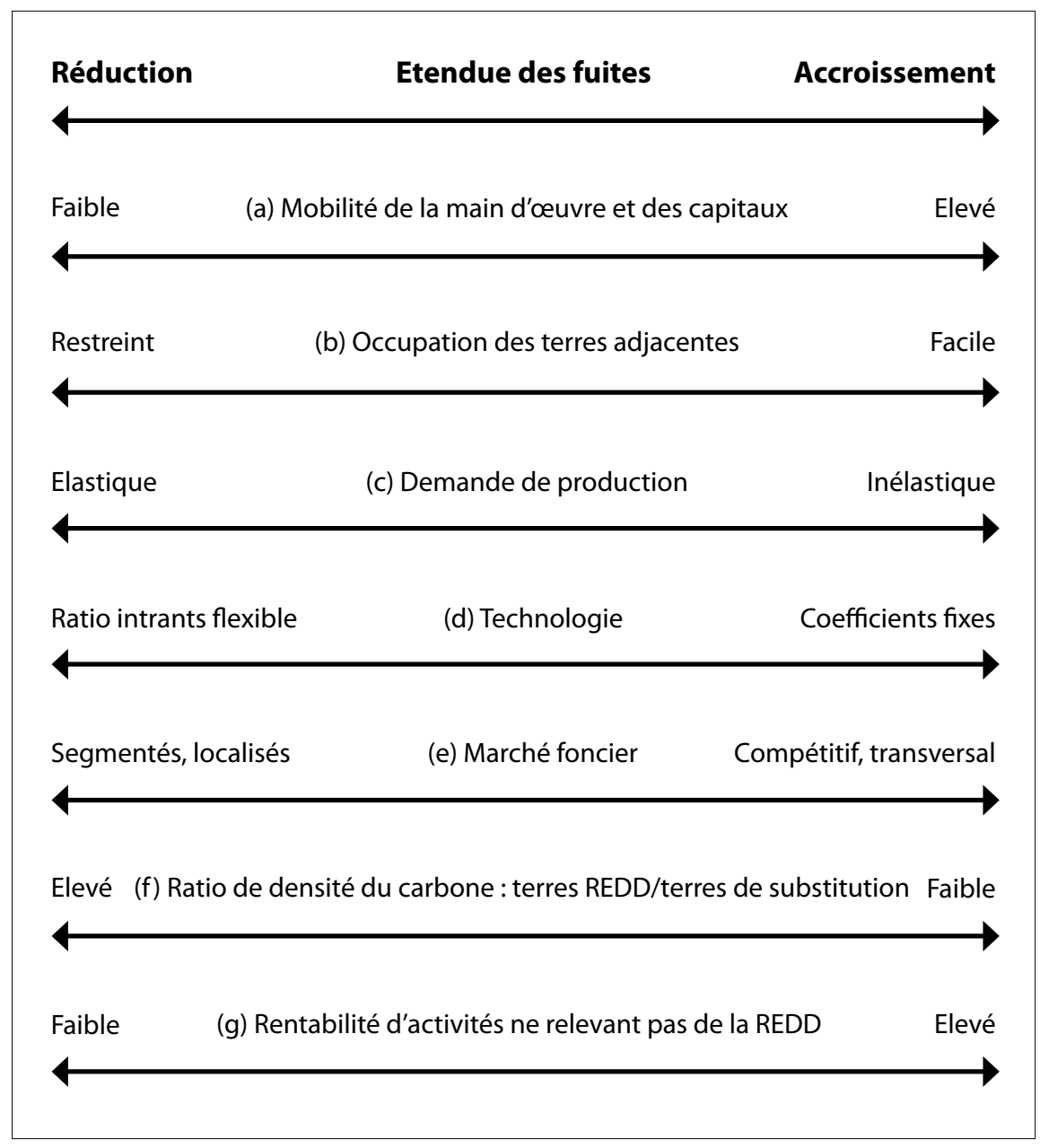

Figure 7.1. Eléments probables d'explication de scénarios à fuites élevées par rapport à des scénarios à fuites faibles

Si les marchés fonciers sont compétitifs et intégrés à toutes les échelles et dans toutes les régions, les fuites sont alors plus probables (e). Par exemple, quand les exploitations de soja se sont développées au Brésil dans les années 1980, les exploitants de soja ont racheté des petites exploitations dans les parties les plus sèches du centre du Brésil, repoussant l'élevage de bétail plus au nord, y compris jusque dans le bassin amazonien. Par contraste, en Papouasie Nouvelle Guinée, pratiquement toute la terre appartient aux communautés locales et des changements d'affectation à grande échelle - sur le modèle brésilien - axés sur le marché, semblent très improbables.

Les fuites concernent aussi les quantités de carbone stockées dans les zones protégées, comparées à celles vers où les activités restreintes pour la REDD se déplacent - y compris les variations dans le temps de stocks de carbone 
comparables (f). Effectivement exclus par la REDD, des secteurs économiques à valeur élevée comme l'exploitation du palmier à huile, du soja, des plantes pérennes ou encore l'abattage et l'extraction minière, seraient sans doute mieux en mesure de couvrir les coûts de transport et de relocalisation sur un autre site que des activités génératrices de revenus moindres comme la récolte de charbon de bois, la pratique de l'agriculture sur brûlis ou l'élevage sur pâturages extensifs (g). Les résultats obtenus après une vérification préliminaire des facteurs (a)-(g) pourraient remettre d'emblée en question certaines propositions de réductions d'émissions de $\mathrm{CO}_{2}$, comme dans le cas Yasuní en Equateur (Encadré 7.2) : même en l'absence de mesures précises, les problèmes liés aux fuites semblent insurmontables.

\section{Encadré 7.2. La proposition Yasuní : Atténuation des émissions de $\mathrm{CO}_{2}$ en laissant le pétrole sous terre}

Le Parc national Yasuní dans la région amazonienne de l'Equateur est doté d'un paysage de forêts d'une extraordinaire biodiversité, mais aussi de très importantes réserves pétrolières. Le Président Correa a annoncé en juin 2007 son intention d'extraire le pétrole du parc de 982,000 ha à moins que la communauté internationale ne lui propose des compensations annuelles de 350 millions de dollars par an pendant 20 ans, ce qui représente à peu près la moitié des recettes estimées du pétrole. Nonobstant les gains sur le plan de la conservation de la biodiversité, l'argument avancé consiste à considérer les bénéfices liés au commerce du carbone comme justifiant quasiment à eux seuls les paiements de compensation demandés : 111 millions de tonnes de carbone qui, autrement, seraient exportées, pourraient rester enfouies dans le sol - ce qui aurait donc pour conséquence que les émissions liées à la dégradation des forêts, au forage et à la mise en place d'une infrastructure routière pour les transports, seraient évitées. Des questions comme celles de la permanence du projet après 20 ans d'existence, ou encore du risque éthique pesant sur un parc établi de longue date mais menacé de perdre son statut de parc national, pourraient alimenter la controverse. Des critères sélectionnés dans la Figure 7.1 montrent aussi que, même si la composante REDD du projet pouvait être efficacement appliquée, les fuites liées au maintien des réserves de pétrole dans le sol avoisineraient les $100 \%$; la demande globale d'énergie étant fortement inélastique et l'offre élastique. Exclure la possibilité d'exploiter le pétrole du parc Yasuní ne ferait donc que déplacer cette activité - à travers des écarts de prix marginaux - vers d'autres sites (c). II suffit de peu de main d'œuvre, les capitaux financiers liés à l'industrie du pétrole sont extrêmement mobiles (a), et les rendements très élevés $(\mathrm{g})$ - ce qui augmente encore la fongibilité du facteur spatial et favorise des fuites globales élevées.

Source : Correa et Moreno (2002)

Une distinction n'est pas toujours établie entre les activités liées à l'exploitation forestière et à l'abattage (facteurs déterminants de la dégradation des forêts) et les activités qui relèvent de la conversion des terres forestières en terres agricoles (facteurs déterminants de déforestation) dans les études sur les fuites liées à la REDD (Murray 2008), alors qu'elles correspondent à des processus économiques sensiblement divergents. L'abattage d'essences à haute valeur 
lucrative est une activité de rente type nécessitant peu d'investissements fixes dans l'espace alors que la déforestation - moins axée sur les exportations constitue normalement, en règle générale, un investissement immobilisant une future utilisation des terres, avec des rendements plus variables. D'après la Figure 7.1, il semblerait que le coefficient de fuites soit plus élevé pour l'abattage d'essences à forte valeur lucrative que pour la déforestation.

Enfin, différentes échelles de fuites servent différents objectifs. Les fuites liées aux exploitations agricoles sont à la base des projets PSE qui sont un outil essentiel pour mettre la REDD en ouvre sur le terrain. Les fuites au niveau $\mathrm{du}$ projet constituent des informations importantes pour les investisseurs même si les niveaux de référence régionaux sont souvent plus fiables (Sathaye et Andrasko 2007). Les approches combinées peuvent être une passerelle pour atteindre des objectifs nationaux (chapitre 9). Des fuites peuvent se produire à l'échelle internationale dans des pays à couverts forestiers denses et à faibles taux de déforestation (par ex., le Gabon et le Surinam) si des incitations préventives modérées ne sont pas proposées à ces pays afin qu'ils protègent leurs importants patrimoines forestiers (da Fonseca et al. 2007) - ce qui relierait les fuites aux niveaux de référence pour la comptabilisation et aux stocks (chapitres 6 et 9).

\subsection{Options pour remédier aux fuites}

\subsubsection{Surveiller}

Les fuites constituent sans aucun doute un « carton rouge " pour la REDD. Fort complexe, la question fait l'objet d'une recommandation extrêmement importante que l'on retrouve dans un grand nombre de soumissions présentées à la Convention-cadre des Nations Unies sur les changements climatiques (CCNUCC) - par ex. celles de la Colombie, de l'Union Européenne, des Etats-Unis d'Amérique (voir Appendice) - à savoir qu'il est essentiel de mieux en surveiller l'ampleur. S'agissant des fuites primaires, les chiffres historiques de déforestation (de préférence, ventilés par secteur) sont d'importance vitale. Une sélection attentive des zones-témoins peut aider à surveiller les impacts à l'intérieur et à l'extérieur des limites spatiales des projets. Des études socio-économiques locales et des indicateurs de tendances (données démographiques, prix des terrains, cultures, production animale et produits ligneux) peuvent aider à mieux comprendre et à mesurer l'impact hors site (Aukland et al. 2003). Des programmes comme The Voluntary Carbon Standard axé sur des projets d'utilisation des terres et le BioCarbon Fund recommandent de surveiller les fuites en lisière du périmètre des projets, par ex. sur des superficies représentant de cinq à sept fois ce périmètre lorsqu'il est supérieur à 100000 ha et le représentant de 20 à 40 fois lorsqu'il est inférieur à 100000 ha. Les fuites secondaires et celles qui se produisent au niveau international doivent être surveillées à travers des modèles économiques ou des échanges 
internationaux qui se fondent sur des bases de données améliorées, afin de pouvoir éventuellement réduire les immenses écarts de prévision actuels et mieux modéliser les situations les plus sensibles (section 7.2).

\subsubsection{Agrandir l'échelle}

Dans un grand nombre de soumissions présentées à la CCNUCC il est recommandé de recourir à des échelles de comptabilisation hiérarchiquement supérieures, c'est-à-dire de passer de l'échelon sous-national à l'échelle nationale afin de mieux maîtriser les fuites (voir chapitre 4). Les fuites qui se produisent au niveau international à travers le marché des produits de base sont potentiellement élevées pour les activités de la REDD qui diminuent sensiblement l'offre globale de matières premières quand la hausse des prix du marché mondial en stimule la production ailleurs. Plus les pays qui déboisent participeront à la REDD, moins il y aura de probabilités de fuites causées par la déforestation au niveau international.

\subsubsection{Utiliser des taux d'actualisation}

Dans certaines soumissions à la CCNUCC (par ex. Colombie, Banque Mondiale - voir Appendice), des doutes ont été exprimés quant à la possibilité de mieux maîtriser les fuites dans la pratique en agrandissant l'échelle de la REDD. Tant que la participation d'un pays reste en-dessous de certains seuils, il pourra être nécessaire d'attribuer les bénéfices liés à la REDD à des conditions moins avantageuses non seulement pour tenir compte du caractère de non permanence mais aussi des fuites estimées au niveau international (Murray 2008). Les divers mécanismes proposés dans le cadre de la CCNUCC comme de constituer des réserves de conservation non créditées, de prendre des assurances, d'actualiser les crédits, ou d'ajuster les niveaux de référence et les objectifs en tenant compte des fuites (Murray 2008), ont essentiellement les mêmes fins que les méthodes de comptabilisation des crédits plus classiques et plus conservatrices. Il sera nécessaire d'améliorer la surveillance pour pouvoir calculer avec précision les taux d'actualisation. Cela pourrait aussi être utile d'en faire un centre d'intérêt des projets de démonstration de la REDD à des fins d'apprentissage.

\subsubsection{Rééquilibrer}

Des questions qui figurent moins souvent dans les soumissions à la CCNUCC mais dont l'importance est tout aussi grande sont celles qui ont trait aux modèles envisagés à l'échelle nationale et à celle des projets (section 7.2.3) : quelle est l'ampleur des risques de fuite pour différentes actions REDD sur le terrain ? Quelle importance respective faut-il accorder, pour maîtriser les 
fuites, à différentes caractéristiques de l'action REDD proposée : objectif principal, site géographique, limites, incitations ? Etant donné les effets très différents que peuvent avoir la conservation, la GDF et les activités B/R sur la REDD (Tableau 7.1), est-il possible de maîtriser les fuites en contrebalançant soigneusement ces effets les uns avec les autres aux échelles nationales et régionales à travers une meilleure absorption de la main d'œuvre et des capitaux exportés (Schwarze et al. 2002) ? Réussir ce délicat exercice d'équilibre pourrait permettre de réduire les fuites au niveau sous-national dans des proportions substantielles.

\subsubsection{Neutraliser}

Quelques simulations de stratégies décisionnelles (Aukland 2003 :129) recommandent de traiter toutes les fuites primaires en neutralisant les composantes liées aux "modes de vie alternatifs ». Cependant, comme nous l'ont appris des décennies d'investissements dans des projets intégrés de conservation et de développement [Integrated Conservation and Development Project (ICDP)], changer les modes de vie peut constituer un immense défi. Si le changement de mode de production, par exemple, exige des éleveurs de bétail brésiliens qu'ils pratiquent une gestion plus intensive de leurs pâturages, il pourrait être souhaitable d'ajouter au projet des incitations et une formation spécifiques. En revanche, s'il s'agit de faire de bûcherons et de paysans pratiquant des cultures itinérantes des opérateurs d'écotourisme et des négociants en produits forestiers non ligneux - comme dans le cas Noel Kempff - la tâche pourrait bien se révéler à la fois trop ardue, trop coûteuse et trop risquée. Il arrive aussi, à l'inverse, que des projets intégrés de ce type (ICDP) réussissent trop bien sur le plan économique et deviennent des " aimants " qui attirent les candidats migrants et augmentent encore les pressions exercées sur les ressources naturelles (Wittemyer et al. 2008). Ainsi la diffusion de technologies intensifiant le rendement des sols - souvent recommandée par les spécialistes pour maîtriser les fuites - peut être si bien accueillie que la déforestation augmente, entraînant ce qui a pu être qualifié d'un " effet d'acceptation exagéré » (Aukland 2003).

\subsection{Evaluation des options}

Si vous enfoncez votre poing dans un oreiller, vous comprimerez quelques plumes mais des renflements se produiront ailleurs. Il en va de même pour les fuites liées à la REDD qu'il est impossible d'éliminer complètement à moins que toutes les forêts et zones boisées du monde participent en même temps à un même programme REDD. Etant donné l'ampleur du phénomène cependant, comment remédier aux fuites à travers des actions qui prennent en compte de façon équilibrée les critères d'efficacité, d'efficience et d'équité ? 
La façon la plus efficace de procéder est sans aucun doute d'agrandir les échelles d'action de la REDD, aussi bien à l'intérieur qu'à l'extérieur des pays. Dans le contexte des stratégies actuelles en matière de climat, et s'agissant des fuites les mesures d'atténuation n'entrant pas ici en ligne de compte - c'est l'échelle mondiale qui constitue la règle. Seule une participation globale élargie peut les réduire et c'est sous cet angle que la REDD est un point d'entrée stratégique. Si la fuite peut être raisonnablement chiffrée à travers la surveillance (7.3.1), il est souhaitable d'actualiser les bénéfices ou d'inscrire des " crédits de réserve " sur des comptes bancaires (7.3.3), en veillant à ce que seules des réductions nettes d'émissions soient récompensées. Réaménager les modèles d'intervention de la REDD peut effectivement restreindre les fuites à l'intérieur d'un pays (7.3.4). Neutraliser les fuites (7.3.5) est un procédé exceptionnellement recommandable ; des projets intégrés de type ICDP rajoutés après-coup risquent de devenir superflus dans le contexte de la REDD.

S'agissant d'efficience des coûts (rentabilité), il y a probablement un niveau de surveillance optimal au-delà duquel il est vain de mesurer les fuites, en particulier celles qui sont dues à la dégradation. Il faut toutefois définir des limites de surveillance explicites. Tenter de neutraliser les fuites peut se révéler souvent plus onéreux que de revoir complètement le modèle du projet ou d'actualiser les crédits. En dépit des complexités, il semble possible d'atteindre l'objectif recherché, à savoir d'arriver à maitriser les fuites avec efficience et efficacité - les risques de fuites ne devant pas nous faire désespérer de la REDD et l'abandonner.

S'agissant d'équité et de préoccupations liées au développement, l'existence de fuites peut en fait indiquer que l'économie est en bonne santé : en réponse aux obstacles mis en place par la REDD, les facteurs de production circulent librement à la recherche de nouvelles opportunités, maintenant les pertes d'acquis sociaux au minimum. Par exemple, si une zone protégée au titre de la REDD empêche la conversion de terres forestières en terres de cultures de soja à fort rendement, il se pourrait qu'il ne soit pas socialement souhaitable d'empêcher les fuites résultant de cette conversion si l'on doit se priver des bénéfices associés à une devise forte et de ses effets multiplicateurs. Même les contrats explicitement liés aux fuites primaires, comme les arrangements conclus en Bolivie empêchant les exploitants forestiers de partir poursuivre leurs activités ailleurs, peuvent ne pas être souhaitables sur le plan des acquis sociaux et du bien-être de toute une communauté. En outre, dans un monde où les capitaux financiers sont extrêmement mobiles, ils pourraient en définitive n'avoir que des effets à court terme. Réaménager la REDD pour la réorienter vers des facteurs moins mobiles et moins susceptibles de provoquer des fuites (par exemple, main d'œuvre, terres marginales) pourrait améliorer l'équité en créant des investissements REDD en faveur des pauvres. Rééquilibrer les zones de conservation de la REDD qui sont réductrices d'activités en les contrebalançant par des interventions de boisement/reboisement (B/R) et de GDF qui sont 
créatrices d'expansion et en puisant dans la panoplie des mesures d'atténuation prévues au titre de la REDD pourrait permettre de contrer l'émigration d'une main d'œuvre en voie de paupérisation. C'est en reconnaissant l'existence de compromis possibles entre l'objectif d'atténuation des émissions de $\mathrm{CO}_{2}$ d'une part et des objectifs de développement plus vastes d'autre part que nous pourrons peut-être arriver à accepter délibérément un certain degré de fuites et réorganiserons les priorités de nos actions d'atténuation. 


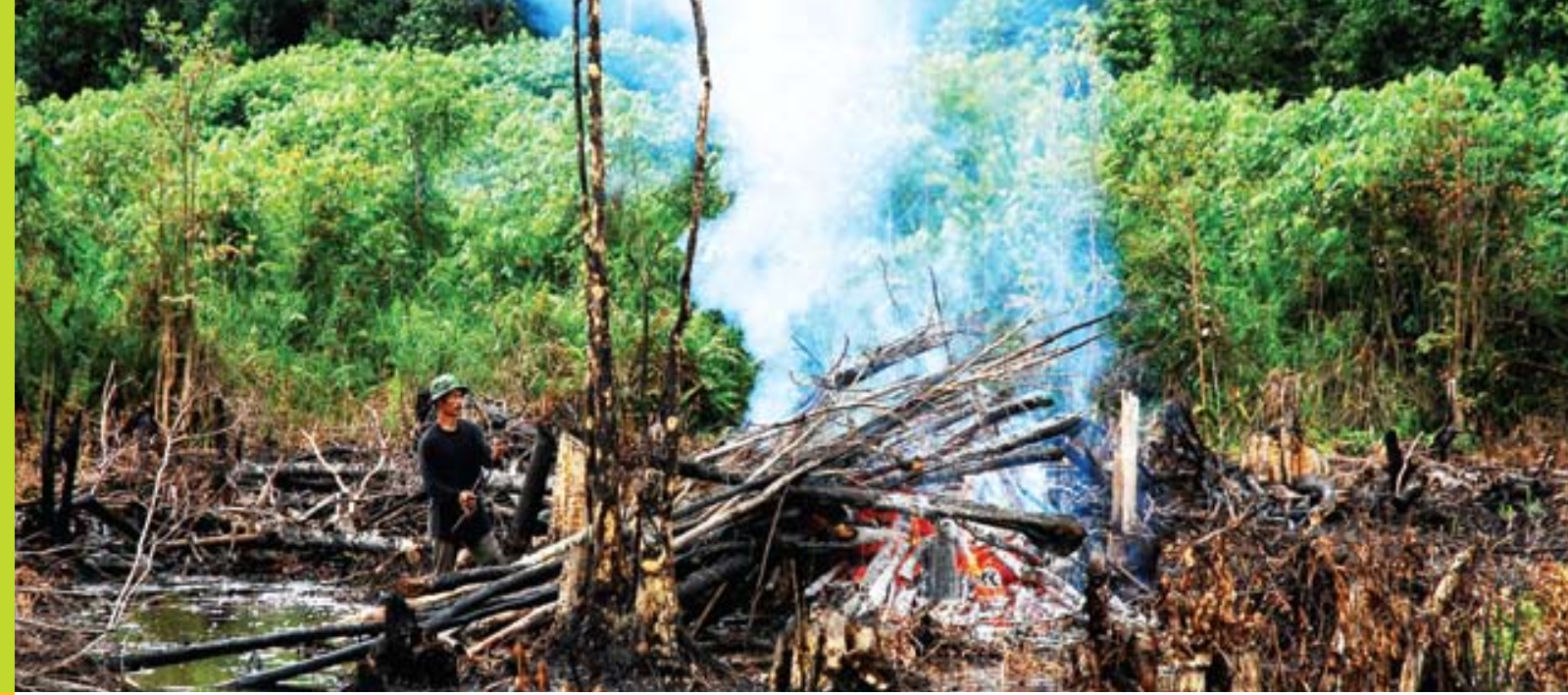

\section{Chapitre 8}

\section{Comment garantir la permanence et déterminer les responsabilités?}

Michael Dutschke en collaboration avec Arild Angelsen

\subsection{Introduction}

L'une des grandes préoccupations du débat sur la réduction des émissions dues à la déforestation et à la dégradation des forêts (REDD) concerne la permanence des réductions d'émissions. Comment faire en sorte qu'un périmètre forestier épargné aujourd'hui ne soit pas détruit demain ? Qui devrait être tenu pour responsable si le cas se produit ? Comment élaborer les contrats et mécanismes de la REDD pour garantir la permanence des réductions ?

Comparéeà d'autres options d'atténuation des effets du changement climatique, la foresterie présente deux aspects particuliers. Tout d'abord, il est plus difficile de mesurer le stockage du carbone. Même quand les meilleures pratiques de gestion sont appliquées, on ne peut exclure une libération de carbone inattendue dans l'atmosphère. Des épisodes de sécheresse ou de destruction dus aux ravageurs, ou encore des feux de forêt, ont un potentiel suffisant pour renverser le processus annuel d'absorption du carbone en quelques semaines ou quelques mois (Schlamadinger et al. 2007). Deuxièmement, l'effet sur le climat d'une activité forestière d'atténuation est lié au maintien des arbres sur le périmètre forestier qui a été vérifié. Un mécanisme REDD efficace doit 
apporter des incitations continuelles aux propriétaires fonciers pour qu'ils surveillent et entretiennent leurs terres forestières.

Il existe au moins trois arguments à opposer à l'établissement d'une distinction catégorique entre la réduction des émissions des combustibles fossiles et la gestion du carbone dans les systèmes terrestres. Tout d'abord, étant donné que les combustibles fossiles sont des ressources qui existent en quantités limitées, il est vraisemblable qu'elles finiront de toute façon par être diluées dans l'atmosphère à plus long terme. L'usage plus réduit que l'on fait de nos jours des réserves de charbon, de pétrole et de gaz préserve une partie de ces ressources (charbon, pétrole et gaz) mais est aussi porteur du risque de production et de consommation plus fort à l'avenir de la partie préservée aujourd'hui. La question de la permanence n'est donc pas limitée à la REDD.

Deuxièmement, même si la séquestration du carbone terrestre n'a été en fait que temporaire, elle continuera à avoir un effet positif sur le climat (voir "l'approche des tonnes par an " étudiée ci-dessous). Dans ce contexte, la REDD peut entraîner rapidement de fortes réductions d'émissions, laisser ainsi le temps de progresser aux technologies et devenir une véritable " passerelle en bois vers un avenir d'énergies propres" (Lecocq et Chomitz 2001). Sans ces mesures d'atténuation liées à des réductions d'émissions dans les forêts, le monde ne pourra probablement pas connaître à bref délai les réductions d'émissions dont il a rapidement besoin pour ne pas atteindre l'élévation de température maximale projetée de $2{ }^{\circ} \mathrm{C}$ (par ex. Stern 2007).

Troisièmement, la déforestation a correspondu - pour la plupart des pays développés du monde d'aujourd'hui - à une phase de leur développement. Dans le contexte des paysages forestiers, on tend plutôt à scinder le processus de transition en phases de transition : d'un épisode de déforestation lente à un épisode de déforestation accéléré à une phase de stabilisation, suivie ultérieurement d'une nouvelle transition caractérisée par un lent accroissement du couvert boisé (Rudel et al. 2005). Un mécanisme REDD efficace permettra de préserver les forêts pendant cette phase délicate de reprise de leur croissance dont une grande partie aura un caractère permanent (Chomitz et al. 2006).

Force est de constater que la question de la permanence - qui ne se limite pas au seul contexte de la REDD - devra cependant être prise en compte dans les négociations sur la réduction des émissions dues à la déforestation et à la dégradation des forêts. Dès le moment où quelqu'un devient responsable d'un stock de carbone terrestre, la non-permanence peut continuer à constituer une menace mais ses effets dommageables pour l'atmosphère seront compensés ce qui pourrait être le cas à l'avenir si les pays en développement deviennent responsables de leurs propres objectifs d'émissions, par exemple dans les limites d'un système de plafonnement et d'échange (Eliasch 2008). Avant que cela puisse se produire cependant, il nous faudra trouver des solutions 
intermédiaires. Dans ce chapitre, nous étudions les différents risques liés à la permanence ainsi que la façon de les gérer et nous proposons donc une «boîte à outils » composée des différents mécanismes de responsabilités nécessaires pour que les crédits carbone liés à l'utilisation des terres et à d'autres secteurs d'activités deviennent fongibles et interchangeables.

\subsection{Risques pour la permanence et comment les gérer}

La permanence des réductions d'émissions peut être mise en péril par un certain nombre de risques directs. A un premier niveau de risques, comment gérer le risque de réémissions à l'échelle des pays ou des projets ? Cependant, si les réductions d'émissions doivent être comptabilisées et utilisées sur des marchés volontaires ou réglementaires de réduction d'émissions de gaz à effet de serre (pour enregistrement ou compensation), il faudra aussi gérer les risques à un deuxième niveau et mettre en place un système de responsabilité commerciale. Les deux niveaux se chevaucheront inévitablement. Ils restent cependant distincts puisque les risques liés à la permanence, dans le cas du premier niveau de risques, devront être gérés de toute façon et indépendamment du fait que des crédits d'émission seront ou non générés tandis qu'il s'agira d'une nécessité commerciale au deuxième niveau, au cas où les crédits REDD seraient commercialisés.

\subsubsection{Risques et gestion des risques}

Quels sont les risques qui peuvent mettre en danger la permanence du carbone stocké dans les forêts? Différents types de risques ont pu être distingués (Wong et Dutschke 2003) :

1. Risque naturel/écologique : Variations aléatoires des stocks de carbone, causés par des événements naturels comme les orages, les sécheresses, les ravageurs ou les incendies.

2. Risque lié au changement climatique : Le changement climatique peut entraîner des pertes de carbone systématiques dans certaines régions. Il s'agit d'un type de risque distinct des autres types de risques naturels/ écologiques parce qu'il implique une nouvelle catégorie de menaces contre lesquelles il pourrait être plus difficile de s'assurer étant donné notre absence d'expérience à cet égard.

3. Risque lié à la demande : Quand la demande de produits agricoles est le principal moteur de la déforestation, une augmentation des prix sur le marché national ou mondial peut faire monter les coûts d'opportunité à 
des niveaux supérieurs aux prix convenus pour le carbone, et rendre ainsi rentable la conversion des forêts en terres agricoles.

4. Echec des projets partenaires : Le risque lié à l'absence de performance d'un projet peut être dû, par exemple, à une gestion inefficace, à des droits de propriété sur la forêt qui ne sont pas garantis (empiètement), ou à la faillite de projets partenaires.

5. Risque politique : Un changement de gouvernement peut entraîner un changement ou un renversement d'approbations ou d'engagements préalables. Il pourrait en aller de même en cas de troubles de l'ordre public. Selon le modèle finalement retenu pour le mécanisme de la REDD, les activités au niveau sous-national pourraient en définitive être aussi influencées par un changement de statut (des pays n'en faisant préalablement pas partie deviennent des pays de l'Annexe I).

En cas d'événements naturels (risque de type 1), l'assurance traditionnellement prise pour les forêts couvre la différence entre la valeur des arbres sur pieds et la valeur commerciale des arbres à maturité. Les contrats sont souvent renouvelés annuellement afin de refléter le profil de risque réel. La couverture pourrait être étendue au carbone fixé dans la végétation, cette extension étant conditionnée par la participation des compagnies d'assurance au marché des réductions d'émissions.

Les variations climatiques à long terme (type 2) ne sont pas uniformément dommageables sur le plan mondial mais elles peuvent avoir un impact négatif sur de vastes superficies tandis que le changement climatique peut entraîner une croissance accrue de biomasse dans d'autres zones. Dans les cas où il est possible d'éliminer tout facteur d'intervention humaine indirecte, ces risques (et bénéfices) ne seront pas attribués aux activités individuelles.

Le risque d'un changement de prix des matières premières (3) peut être partagé entre une institution de financement et un propriétaire foncier en incluant une clause d'indexation dans le contrat qui prévoit des paiements additionnels durant les périodes où les prix de produits comme le soja ou l'huile de palme, par exemple, varient dans des fourchettes qui sont en dehors d'un corridor de prix prédéterminé.

$\mathrm{Au}$ cas où les titulaires du projet ne rempliraient pas leurs obligations ou disparaîtraient (4) et où des crédits permanents auraient été générés, la responsabilité ultime retomberait sur le gouvernement et, plus probablement, sur celui du pays vendeur. Afin de pouvoir répondre à ce risque, le point focal national REDD pourrait demander une prime au risque en nature (par ex., une clause de partage de crédit) avant d'approuver une activité sous-nationale.

Les risques politiques (5) peuvent être minimisés à travers une large participation à l'agenda climatique et par la coopération internationale. Dans le contexte 
d'un accord international comme celui de la Convention-cadre des Nations Unies sur les changements climatiques (CCNUCC) néanmoins, le principe de base est que les Etats sont permanents et respectent les dispositions des Traités. Les possibilités juridiques d'obliger les Etats à appliquer la loi sont forcément limitées.

\subsubsection{Gestion des responsabilités}

Les risques liés à la permanence s'appliquent indépendamment de toute commercialisation des crédits carbone dans le cadre d'un futur mécanisme pour la REDD. Dans le cas de l'approche nationale, le problème n'est plus celui de la permanence de périmètres forestiers particuliers mais celui de savoir si le pays tout entier continuera à appliquer des réductions inférieures au niveau de référence établi, sans tenir compte de l'origine des émissions. Il en découlera immédiatement cette autre question critique : que se passera-t-il si le pays dépasse son niveau de référence ? La réponse pourrait être que le pays compensera les réductions ou s'acquittera de ses obligations en payant une pénalité sous une autre forme. Dans le cas d'un "système de débit " par exemple, toute émission supérieure au niveau de référence pourrait être déduite d'un compte futur (en y ajoutant éventuellement les intérêts ou une autre pénalité). Les émissions supplémentaires devront ensuite être compensées avant que puissent être prises en compte et comptabilisées les réductions d'émissions inférieures au niveau de référence qui pourraient être réalisées à l'avenir (Schlamadinger et Johns 2006).

Toutefois, avant que les pays participant à la REDD puissent accepter l'entière responsabilité des réductions ou que les crédits REDD provenant d'activités sous-nationales acquièrent un caractère fongible et puissent être échangés avec d'autres crédits liés à des mesures d'atténuation ou des droits d'émission, les risques commerciaux en résultant doivent être pris en compte et faire l'objet de garanties. Plusieurs options existent à cet égard, notamment :

1. Une comptabilisation temporaire qui soumet la validité des crédits carbone liés à l'utilisation des terres à la rémanence des stocks de carbone (Blanco et Forner 2000). C'est l'approche adoptée dans le cadre des activités de boisement/reboisement $(\mathrm{B} / \mathrm{R})$ du mécanisme de développement propre (MDP). Selon les modalités prévues, les réductions d'émissions devront être soit certifiées de nouveau soit réactivées après cinq ans pour que le crédit reste valable. Dans le cas du MDP, à l'échéance du projet (60 ans maximum) ou en cas de pertes prématurées, les crédits doivent être remplacés par d'autres types de permis d'émissions. Au titre des dispositions actuelles du MDP, l'inscription de crédits temporaires crée toujours un débit futur, quelle que soit la destination finale des stocks de carbone constitués.

2. L'approche qualifiée "d'approche des tonnes par an » a été étudiée dans le Rapport spécial du Groupe intergouvernemental d'experts sur le changement 
climatique (GIEC) sur l'utilisation des terres, les changements d'affectation et la foresterie (Watson et al. 2000). Le GIEC n'a pas retenu l'idée que (i) la valeur actuelle de l'atténuation est plus élevée aujourd'hui que le même effet de cette atténuation demain, ni que (ii) le temps de rémanence du $\mathrm{CO}_{2}$ dans l'atmosphère est limité. Choisissant de préférence une durée de vie humaine et l'associant à la période de décomposition naturelle, divers auteurs ont calculé une "période d'équivalence ", après laquelle l'atténuation pourrait être considérée comme permanente. Certains ont proposé une durée de 42 à 100 ans pour cette période d'équivalence (Fearnside et al. 2000 ; Moura Costa et Wilson 2000 ; Fearnside 2002). Supposant donc une période d'équivalence de 100 ans, maintenir 100 tonnes de $\mathrm{CO}_{2}$ hors de l'atmosphère pendant plus d'une année, équivaudrait à 1 tonne de $\mathrm{CO}_{2}$ éliminée de façon permanente. Ce type de comptabilisation présente cependant un gros défaut sur le plan de la trésorerie : le paiement total au titre de la réduction permanente n'est effectué qu'à la fin de la période d'équivalence, tandis que les coûts sont essentiellement encourus avant. Le secteur privé pourrait cependant être séparément disposé à avancer les prêts nécessaires sur la base de la valeur du crédit carbone du projet et des paiements à venir.

3. Crédits régulateurs pour le projet. Autre option utilisée dans des projets d'atténuation volontaires. Seule une partie (par ex. $50 \%$ ) des crédits générés est vendue, tandis que le reste est mis en dépôt fiduciaire pour une période prédéterminée (par ex. 50 ans). Une partie de ces crédits est libérée quand cesse la période de garantie s'il n'y a pas eu de pertes.

4. Mise en commun des risques. Il s'agit d'une variante des crédits régulateurs dans laquelle un crédit régulateur conjoint est établi au titre de plusieurs projets - ce qui minimise le risque de dommages survenant simultanément. Les crédits régulateurs de projets individuels peuvent être moins importants que les crédits régulateurs de projets n'ayant pas été mis en commun. Il en irait de même pour un programme REDD au niveau national dans lequel les risques sont répartis entre secteurs d'activités et régions dans le pays tout entier.

5. Assurance. Version avancée de la mise en commun des risques. Un assureur tiers sélectionne un portefeuille de projets assurés de façon à couvrir plusieurs régions de croissance et d'écosystèmes, limitant ainsi le risque de dommages à grande échelle qui surviendraient simultanément. Le paiement de la prime de risque est effectué en unités de réduction d'émissions. En cas de dommage, la compagnie d'assurances remplace les crédits perdus par ceux qu'elle détient en stock. Le risque résiduel est garanti par des instruments financiers et les réassureurs (Subak 2003). Cette option peut aussi permettre d'améliorer la trésorerie destinée aux activités d'atténuation.

6. Responsabilités partagées ou partenariats pour respecter les obligations en matière forestière (FCP). Il s'agit d'une proposition visant à gérer les responsabilités encourues au niveau national par deux pays (ou davantage) 
visés et non visés par l'Annexe I dans le cadre d'une approche de comptabilisation - pour gérer l'utilisation des terres - (Dutschke et Wolf 2007). Le concept élaboré est de faire porter aux pays développés une part de responsabilité négociée de la permanence des crédits REDD une fois que ces crédits sont certifiés. Ces crédits pourraient correspondre au secteur de l'utilisation des terres et leur objectif sectoriel, stipulé à l'Article $3 \mathrm{du}$ Protocole de Kyoto, paragraphes 3 et 4, ou à tout nouvel accord qui aurait été convenu. Ce qui est suggéré au titre du FCP est de consentir un accès préférentiel à des crédits REDD à un pays développé pour avoir respecté ses obligations s'il partage les responsabilités. S'agissant du respect des objectifs de l'Annexe I, on suppose que certaines restrictions affecteront l'utilisation des crédits REDD. Les bailleurs d'aide seraient aussi plus motivés pour investir dans la gouvernance forestière. Le financement bilatéral serait orienté vers les politiques et mesures les plus efficaces pour réduire les émissions dans le secteur forestier. La relation spéciale existant entre les pays participant à la REDD et l'Annexe I aura aussi des répercussions sur le secteur privé parce que c'est grâce à la participation étrangère que le FCP limitera le risque encouru par un pays pour des activités engagées au niveau sous-national.

Les options énumérées ci-dessus peuvent se combiner les unes avec les autres de plusieurs façons. Par exemple, les options 1 et 2 peuvent être combinées avec une annulation progressive des débits encourus au titre des crédits temporairement attribués (Dutschke 2002), améliorant ainsi la trésorerie destinée aux activités d'atténuation. Tout crédit forestier temporairement attribué devra être remplacé ultérieurement mais un pourcentage au prorata de ce futur débit est épongé chaque année jusqu'à échéance de la période d'équivalence si aucun dommage ne survient.

A l'exception de la première, toutes les autres options limitent les responsabilités pendant une période prédéterminée. L'approche dite du " tonnage par an » prend en compte les effets des mesures d'atténuation forestières après la période d'équivalence. Les stocks régulateurs de crédits et les assurances dégagent les crédits du dépôt fiduciaire quand aucun dommage ne survient pendant un certain nombre d'années. Dans le cas des projets de boisement/reboisement (B/R) du MDP de la première période d'engagement, aucun horizon temporel n'a pu être fixé en matière de risques liés au carbone séquestré et c'est un système d'attribution temporaire des crédits qui a été choisi, supposant toute atténuation perdue à l'issue du projet. Cette supposition a cependant fortement diminué l'appétit du marché pour les réductions d'émissions temporaires certifiées et à long terme. Comme le prix des crédits temporaires donne une indication de la valeur future des unités de remplacement, ces crédits sont très spéculatifs et perdent leur valeur si le marché s'attend à des objectifs plus contraignants pendant les périodes d'engagement suivantes. Cette situation pourrait toutefois changer si des dispositions sont prises lors de futures 
périodes d'engagement pour donner des signaux de marché stables et autoriser l'inscription des crédits en banque.

Avec un objectif de limiter le réchauffement global à $2{ }^{\circ} \mathrm{C}$ d'ici le milieu du $21^{\mathrm{e}}$ siècle, le calendrier des mesures d'atténuation est beaucoup plus clair maintenant qu'il ne l'était quand avaient été discutées les règles et les modalités des activités de boisement/reboisement (B/R) du MDP. Toutes les options non retenues à l'époque pourraient donc maintenant être de nouveau prises en compte dans le cadre de la gestion des responsabilités dévolues à la REDD.

\subsection{Evaluation de la gestion des responsabilités}

L'assignation des responsabilités est une condition préliminaire de la fongibilité des crédits. Indépendamment du mode de financement proposé au titre des mesures envisagées pour la REDD, le critère de l'efficacité environnementale s'appuie nécessairement sur le caractère permanent de l'effet de ces mesures, à savoir la réduction des niveaux d'émission de GES dans l'atmosphère. Diverses mesures envisagées pour sauvegarder le caractère permanent des réductions d'émissions et de l'absorption du carbone dans les systèmes terrestres sont énumérées au Tableau 8.1 et chacune d'entre elles est évaluée en fonction du critère des $3 \mathrm{E}$ utilisé dans ce livre (efficacité, efficience et équité). Les options (ou mesures envisagées) 1 et 2 évitent de faire clairement référence à toute assignation de responsabilités et ne sont donc optimales à l'aune d'aucun de ces trois critères d'évaluation. Pendant la phase de démarrage d'une approche combinée (chapitre 4), le recours à un système de crédits temporaires peut être utile avant la mise en place des objectifs nationaux de la REDD et la détermination de la responsabilité ultime du pays. Ensuite les crédits pourront être convertis de crédits temporaires en crédits permanents. Dès que la responsabilité ultime du pays a été déterminée, comme dans le cas des pays visés à l'Annexe 1 , toute réémission sera inscrite dans l'inventaire national et prise en compte au moment de la mise en conformité avec les engagements pris au titre de la réduction des émissions.

Les options énumérées ne constituent pas une liste exclusive ; elles peuvent être considérées comme une suite logique une fois qu'un certain volume d'activités a été atteint. S'agissant des options 4 et 5, il pourrait arriver que les responsables des mises en commun trient sur le volet les "bons risques ". Les pays de l'Annexe I devraient envisager d'apporter des capitaux de lancement pour créer des fonds de réserve plus importants, les rendre accessibles aux pays perçus comme étant "à hauts risques ", ou collaborer avec ces pays pour estomper l'image ainsi projetée. L'option 6 qui ne concerne que le niveau national est complémentaire de toutes les autres options. Tout d'abord, elle sécurise les 
investisseurs et les acheteurs potentiels du fait que l'ultime responsabilité en matière de crédits est confiée à un système qui s'appuie sur l'Annexe I et minimise donc les risques politiques. En outre, elle a le potentiel suffisant pour accroître l'efficacité des politiques et mesures dans le secteur de l'utilisation des terres et pour promouvoir les investissements privés axés sur la REDD. Elle est aussi équitable puisqu' elle peut contribuer à rendre plus attrayants des pays qui, autrement, auraient des difficultés à obtenir des investissements au titre de la REDD étant donné les risques politiques qu'ils présentent.

Tableau 8.1. Options pour assurer la permanence dans la gestion du carbone terrestre

\begin{tabular}{|c|c|c|c|c|}
\hline & & Efficacité & Efficience & Equité \\
\hline 1 & $\begin{array}{l}\text { Comptabilisation } \\
\text { temporaire }\end{array}$ & $\begin{array}{l}\text { FAIBLE } \\
\text { Option de } \\
\text { démarrage pour de } \\
\text { petites quantités } \\
\text { de carbone et des } \\
\text { activités isolées }\end{array}$ & $\begin{array}{l}\text { FAIBLE } \\
\text { Utilisation } \\
\text { minimale en raison } \\
\text { d'une comptabilité } \\
\text { complexe, de coûts } \\
\text { de transaction } \\
\text { élevés et de crédits } \\
\text { de faible valeur }\end{array}$ & $\begin{array}{l}\text { FAIBLE } \\
\text { Des coûts de } \\
\text { transaction élevés } \\
\text { bénéficient aux } \\
\text { projets les plus } \\
\text { importants }\end{array}$ \\
\hline 2 & $\begin{array}{l}\text { Comptabilité du } \\
\text { tonnage par an }\end{array}$ & $\begin{array}{l}\text { FAIBLE } \\
\text { Paiements } \\
\text { d'avance } \\
\text { élevés et valeurs } \\
\text { actuelles nettes } \\
\text { faibles (qui } \\
\text { dépendent } \\
\text { des taux } \\
\text { d'actualisation) } \\
\text { incitations en } \\
\text { nombre limité }\end{array}$ & $\begin{array}{l}\text { FAIBLE } \\
\text { Entraîne des taux } \\
\text { d'actualisation très } \\
\text { élevés, ce qui crée } \\
\text { des difficultés de } \\
\text { trésorerie }\end{array}$ & $\begin{array}{l}\text { FAIBLE } \\
\text { Des coûts de } \\
\text { financement } \\
\text { élevés excluent } \\
\text { les participants les } \\
\text { plus pauvres }\end{array}$ \\
\hline 3 & $\begin{array}{l}\text { Crédits régulateurs } \\
\text { pour le projet }\end{array}$ & $\begin{array}{l}\text { MOYENNE } \\
\text { L'efficacité dépend } \\
\text { de la crédibilité } \\
\text { du projet et de la } \\
\text { gestion des stocks } \\
\text { régulateurs }\end{array}$ & $\begin{array}{l}\text { FAIBLE } \\
\text { Pourcentage élevé } \\
\text { de crédits non } \\
\text { comptabilisés, } \\
\text { trésorerie lente à } \\
\text { venir }\end{array}$ & $\begin{array}{l}\text { ELEVEE } \\
\text { Mise en œuvre } \\
\text { facile et } \\
\text { transparente }\end{array}$ \\
\hline 4 & $\begin{array}{l}\text { Mise en commun } \\
\text { des risques }\end{array}$ & $\begin{array}{l}\text { MOYENNE A } \\
\text { ELEVEE } \\
\text { Instrument efficace, } \\
\text { en fonction de } \\
\text { la taille et de la } \\
\text { composition du } \\
\text { stock régulateur }\end{array}$ & $\begin{array}{l}\text { MOYENNE A } \\
\text { ELEVEE } \\
\text { Dimensions } \\
\text { relativement plus } \\
\text { petites du stock } \\
\text { régulateur }\end{array}$ & $\begin{array}{l}\text { MOYENNE } \\
\text { Capacités } \\
\text { d'organisation } \\
\text { requises; risque } \\
\text { de faire cavalier } \\
\text { seul mais assez } \\
\text { équitable }\end{array}$ \\
\hline
\end{tabular}




\begin{tabular}{|c|c|c|c|c|}
\hline & & Efficacité & Efficience & Equité \\
\hline 5 & $\begin{array}{l}\text { Assurance } \\
\text { commerciale }\end{array}$ & $\begin{array}{l}\text { ELEVEE } \\
\text { Responsabilité } \\
\text { externalisée ; } \\
\text { instrument pour } \\
\text { marchés arrivés } \\
\text { à maturité ; peu } \\
\text { d'obstacles }\end{array}$ & $\begin{array}{l}\text { ELEVEE } \\
\text { Coûts de } \\
\text { transaction faibles } \\
\text { à travers une } \\
\text { évaluation et une } \\
\text { gestion des risques } \\
\text { externalisées }\end{array}$ & $\begin{array}{l}\text { MOYENNE } \\
\text { Peut être } \\
\text { équitable si de } \\
\text { « mauvais risques » } \\
\text { socialement } \\
\text { souhaitables sont } \\
\text { subventionnés }\end{array}$ \\
\hline 6 & $\begin{array}{l}\text { Responsabilité } \\
\text { partagée }\end{array}$ & $\begin{array}{l}\text { ELEVEE } \\
\text { Donnera une } \\
\text { impulsion } \\
\text { supplémentaire } \\
\text { (sous forme } \\
\text { d'incitations) } \\
\text { à la mise en } \\
\text { place d'une } \\
\text { infrastructure et } \\
\text { au renforcement } \\
\text { des capacités, } \\
\text { préparant ainsi le } \\
\text { terrain pour une } \\
\text { REDD efficace }\end{array}$ & $\begin{array}{l}\text { ELEVEE } \\
\text { Permettra d'assurer } \\
\text { les activités liées à } \\
\text { la REDD, si le risque } \\
\text { pour le pays est } \\
\text { minimisé }\end{array}$ & $\begin{array}{l}\text { ELEVEE } \\
\text { Dépend des } \\
\text { motivations des } \\
\text { pays de l'Annexe } \\
\text { I concernés, peut } \\
\text { contribuer à } \\
\text { promouvoir les } \\
\text { investissements } \\
\text { dans les pays à } \\
\text { hauts risques }\end{array}$ \\
\hline
\end{tabular}

\subsection{Conclusion}

Que ces activités se déroulent dans les forêts ou ailleurs, la constitution, la gestion et la conservation des bassins de carbone sont porteuses du risque de non-permanence. Ce risque doit être pris en compte dans toutes les actions visant à atténuer les effets du changement climatique. Il sera aussi nécessaire d'avoir recours à des mécanismes de responsabilisation pour assurer la fongibilité des crédits (qui seront attribués au titre des mesures d'atténuation prises dans le cadre de la REDD) avec les autres crédits et permis d'émission. Les risques liés aux stocks de carbone forestier peuvent être atténués grâce à une approche par paliers, différents mécanismes s'appliquant à diverses strates de risques. La responsabilité nationale ultime de chaque pays participant à la REDD en cas d'échec des stratégies d'atténuation constitue indubitablement le mécanisme le plus efficace dans le contexte de la mise en commun des risques. Comme les gouvernements participant à la REDD n'ont pas (encore) d'objectifs de réduction d'émissions de GES pour l'ensemble de leur économie, ils ne sont pas en mesure de compenser transversalement de mauvais résultats en matière de foresterie par des résultats supérieurs aux objectifs fixés dans un autre secteur. Des responsabilités partagées d'un secteur à l'autre entre pays développés et pays en développement pourraient ainsi contribuer à la stabilité du système de la REDD. Pour chacun des partenaires respectifs d'un pays développé, le bénéfice pourrait être d'avoir un accès préférentiel aux crédits du partenaire REDD concerné. 
Dans ce chapitre, nous avons dressé une liste récapitulative des outils proposés pour réduire les risques liés au carbone dans le secteur forestier et pour sécuriser les contrats conclus sur l'utilisation du carbone résultant des activités d'atténuation liées aux forêts. Cette boîte à outils est l'aboutissement d'un projet pilote de développement et du débat méthodologique très animé qui se déroule depuis plus d'une décennie dans le cadre de la CCNUCC. Il devrait être possible de garantir la permanence (des réductions d'émissions) et d'assigner les responsabilités en fonction d'un mécanisme REDD qui combinerait toute une série d'approches complémentaires les unes des autres. La décision qui sera prise à Copenhague 2009 sur la REDD devrait offrir toute une diversité de choix possibles basés sur ce qui permettra de tenir le mieux compte des circonstances nationales. 

préparé des projets pilote et de démonstration. Ces groupes ont beaucoup fait avancer l'application des technologies de télédétection.

Il est question dans ce chapitre de l'utilisation des capacités MSV pour réduire les émissions dues à la déforestation et à la dégradation des forêts dans les pays en développement (REDD). Nous décrivons succinctement l'état d'avancement de la science dans le domaine de la surveillance, du suivi et de la vérification du carbone, notre objectif étant de montrer qu'une grande partie des questions qui se posaient d'un point de vue méthodologique en 2001 ne représentent plus une contrainte pour ces types de projets. Nous sommes convaincus que les progrès réalisés au cours des sept dernières années ont créé un nouvel environnement politique plus favorable à l'instauration de projets liés à la REDD grâce auquel d'autres techniques encore plus novatrices permettront d'accroître la faisabilité de projets pour réduire une des sources substantielles d'émissions de gaz à effet de serre dans l'atmosphère.

\subsection{Compromis coût - précision (des mesures)}

Différentes méthodes sont disponibles et peuvent adéquatement surveiller la déforestation, la dégradation des forêts et les stocks de carbone. S'agissant de la déforestation, la surveillance peut être effectuée par télédétection avec des mesures au sol pour vérification. Il est plus difficile de surveiller la dégradation des forêts et l'évolution des stocks de carbone qui dépendent largement de mesures au sol complétées par la télédétection.

Il n'en reste pas moins qu'il serait judicieux de trouver un compromis entre le coût et la précision des mesures. L'exactitude des mesures est essentielle pour assurer que les réductions d'émissions ne sont ni surévaluées ni sousévaluées et que les paiements effectués au titre des efforts de réduction sont adéquats. Dans certains contextes nationaux, des niveaux de précision élevée exigent le recours à des techniques d'imagerie à fine résolution (par ex. pour détecter une dégradation ou une déforestation de petite envergure), ou à des imageries répétées dans le temps (par ex. pour surmonter les difficultés liées à la couverture nuageuse) ou à une imagerie dont le traitement requiert davantage de compétences techniques (par ex., l'analyse des images radar) - toutes ces techniques étant onéreuses. De même, les mesures au sol, cruciales pour les opérations de vérification et de mesure des stocks de carbone, prennent du temps et sont relativement coûteuses pour des applications à grande échelle comme les inventaires établis au niveau national (Korhonen et al. 2006).

Il est d'autant plus important de trouver un compromis entre le coût et la précision des mesures que les pays qui ont besoin de méthodes de surveillance onéreuses (en raison de particularités tenant à la couverture nuageuse ou au terrain accidenté ou encore aux facteurs déterminants) tendent à être les mêmes 
que ceux qui n’ont pas les capacités suffisantes pour satisfaire à ces besoins. La reconnaissance de la nécessité d'un tel compromis a incité la plupart des parties à la CCNUCC à solliciter la communauté internationale pour définir des orientations sur des méthodes rentables pour mesurer, suivre et vérifier les réductions d'émissions dues à la déforestation et à la dégradation des forêts.

Des lignes directrices officielles restent encore à établir pour le MDP de la REDD. Les lignes directrices des bonnes pratiques 2003 pour les activités d'utilisation des terres, de changements d'affectation et de foresterie (GBPLULUCF) et les lignes directrices 2006 pour les inventaires nationaux des gaz à effet de serre pour l'agriculture, la foresterie et autres utilisations des terres (AFOLU) - toutes deux élaborées par le GIEC - sont des premières étapes importantes mais les méthodes doivent encore être étoffées pour pouvoir estimer les émissions liées aux forêts, notamment en ce qui concerne le modèle d'échantillonnage et la détermination des densités du carbone dans les forêts dégradées (CCNUCC 2008b). Le groupe de travail ad hoc de la REDD the Global Observation of Forest and Land Cover Dynamics (GOFC-GOLD) - a entrepris une première démarche pour répondre à ce besoin en préparant un manuel qui donne une perspective consensuelle - associant les experts du carbone et ceux de la communauté mondial d'observation de la terre - des questions méthodologiques ayant trait aux activités de la REDD au niveau national (GOFC-GOLD 2008).

\subsection{Eléments constitutifs d'un système de surveillance et de mesure}

Etant donné la nécessité de trouver un compromis entre le coût et la précision des mesures, la recherche de solutions rentables est au cœur du débat des capacités à surveiller, rendre compte et contrôler, (Monitoring, Reporting and Verification, MRV). Un système de surveillance et d'évaluation rentable pour la REDD suppose l'adoption d'une approche équilibrée combinant techniques de télédétection et mesures au sol. Les techniques d'imagerie aident à mettre au point des modèles d'échantillonnage au sol efficaces (par ex. dans les zones à forte variabilité), à évaluer les variations de superficie (avec vérification au sol) et à extrapoler les mesures des parcelles aux échelles régionale ou nationale. Les mesures au sol sont nécessaires pour mesurer les stocks de carbone et pour vérifier les représentations cartographiées des forêts à partir des images satellite.

Les émissions de $\mathrm{CO}_{2}$ liées à la déforestation et à la dégradation des forêts sont estimées à partir des variations de deux variables importantes : (i) les superficies déboisées et dégradées; et (ii) les densités des stocks de carbone par unité de surface. Les technologies de télédétection associées aux mesures au sol jouent un rôle clé dans la surveillance de ces variables. 


\subsubsection{Surveillance des zones de déforestation}

La télédétection est la seule méthode pratique pour surveiller la déforestation au niveau national (DeFries et al. 2006). Depuis le début des années 1990, les variations de superficies forestières ont été surveillées depuis l'espace avec un certain degré de confiance (Achard et al. 2008). Certains pays (par ex. le Brésil et l'Inde) ont des systèmes opérationnels bien établis depuis plus d'une décennie tandis que d'autres les mettent en place ou ont pu surveiller leurs forêts avec succès grâce à des photos aériennes qui ne nécessitent ni des analyses de données ni des moyens informatiques très complexes (DeFries et al. 2006).

Les deux approches les plus habituelles sont la cartographie complète et l'échantillonnage. La cartographie complète qui permet de surveiller soit un pays tout entier, ou plus simplement une forêt, sont communément utilisées aussi bien au Brésil qu'en Inde. Les approches par échantillonnage sont utiles pour diminuer les coûts de collecte et d'analyse des données et conviennent tout particulièrement aux processus de déforestation concentrés dans des parties discrètes d'un pays ou d'une région. Au nombre des méthodes d'échantillonnage recommandées figurent l'échantillonnage systématique avec prélèvement d'échantillons à intervalles réguliers (tous les $10 \mathrm{~km}$ ) et l'échantillonnage stratifié, avec détermination des échantillons par des variables approximatives connues (par ex., zones critiques de déforestation) (Achard et al. 2008). Des informations techniques et scientifiques peuvent aussi aider à déterminer les priorités en matière d'échantillonnage (DeFries et al. 2006) comme dans le cas d'une méthode d'échantillonnage stratifié, utilisée par exemple dans le projet de surveillance de la forêt tropicale ombrophile amazonienne du Brésil (Projeto Monitoramento da Floresta Amazônica Brasileira por Satélite - PRODES), qui a identifié des « zones critiques " sur la base des résultats des opérations de surveillance de l'année précédente visant à déterminer les priorités d'analyse de l'année suivante (INPE 2004).

Une approche n'exclut pas l'autre : une méthode d'échantillonnage utilisée pendant une période de surveillance peut être étendue à la couverture complète de la couverture végétale et d'occupation des sols à la période suivante. De même, la cartographie de la couverture végétale à grande échelle pendant une période peut être suivie par une analyse des zones critiques (échantillonnage stratifié) la période suivante.

Une approche progressive, étape par étape, devrait permettre de diminuer les coûts. Le premier pas (ou la première étape) consistera à analyser des données de résolution brutes (par ex. MODIS) pour déterminer les sites affectés de taux de variation élevés de changements d'affectation des terres (points critiques de déforestation). Dans un deuxième temps, des données plus coûteuses à résolution moyenne (par ex. Landsat, SPOT, SAR) seront utilisées pour conduire une analyse détaillée de ces points critiques. L'approche étape par étape diminue le besoin d'une analyse globale de toutes les zones de forêts 
d'un pays. Hansen et al. (2008) par exemple ont utilisé cette méthodologie au niveau mondial pour calculer les taux de défrichement des forêts tropicales humides entre 2000 et 2005.

La précision des relevés et la vérification des résultats sont des éléments constitutifs essentiels de tout système de surveillance. Des taux de précision de 80 à $95 \%$ peuvent être obtenus dans les cas où la surveillance est effectuée avec des techniques d'imagerie à moyenne résolution (par ex. Landsat) pour distinguer les zones boisées de celles qui ne le sont pas. La précision peut être estimée par des observations au sol ou à travers des analyses à fine résolution (instruments embarqués à bord d'aéronefs ou imageries satellite). Les photographies aériennes restent un bon outil de vérification étant donné le coût élevé de l'imagerie à fine résolution. Il existe toutefois une source d'images gratuites (jusqu'à $50 \mathrm{~cm}$ de résolution) - celle de Google Earth qui, là où elle est disponible, fournit des données continuellement actualisées (Olander et al. 2008). ${ }^{1}$

\subsubsection{Surveillance des zones forestières dégradées}

Les causes de dégradation dans les forêts sont attribuables à une série de facteurs qui ont une incidence sur les conditions dans lesquelles la surveillance s'effectuera (Tableau 9.1 ; voir aussi le chapitre 10 sur la dégradation). Une surveillance répétée est nécessaire pour veiller à ce que toutes les variations du paysage forestier soient prises en compte et puissent être attribuées à une période de temps déterminée. L'utilisation de techniques de télédétection pour stratifier les terres afin de sélectionner les périmètres où seront effectuées des mesures au sol a été proposée afin de permettre de relever les défis associés à la dégradation qui n'est pas encore clairement définie.

Tableau 9.1. Causes de dégradation et impact sur la surveillance

\begin{tabular}{ll}
\hline $\begin{array}{l}\text { Causes de } \\
\text { dégradation des } \\
\text { forêts }\end{array}$ & Surveillance de la faisabilité \\
\hline Abattage sélectif & $\begin{array}{l}\text { - Les méthodes de télédétection utilisant des techniques } \\
\text { d'imagerie à moyenne résolution permettent de déceler } \\
\text { les trouées dans la canopée qui sont causées par } \\
\text { l'infrastructure routière et les aires de stockage des grumes } \\
\text { - La réduction des stocks de carbone peut aussi être estimée } \\
\text { sans imagerie satellite en utilisant les méthodes GL-AFOLU } \\
\text { du GIEC 2006, bien qu'il soit probablement plus difficile } \\
\text { d'estimer les émissions résultant de l'abattage }\end{array}$ \\
\end{tabular}

Bien que l'image ne puisse pas être entièrement importée et traitée, elle a un grand intérêt pour la validation des cartes en associant l'interprétation visuelle avec les polygones géodésiques (SIG) et les coordonnées des points qui peuvent être importés et superposés sur Google Earth (Olander et al. 2008). 


\begin{tabular}{ll}
\hline $\begin{array}{l}\text { Causes de } \\
\text { dégradation des } \\
\text { forêts }\end{array}$ & Surveillance de la faisabilité \\
\hline Feux de forêts & - $\begin{array}{l}\text { Plus difficiles à surveiller avec les imageries satellite } \\
\text { existantes, mais la REDD peut s'appuyer sur les rapports } \\
\text { concernant les feux de forêts }\end{array}$ \\
\hline $\begin{array}{l}\text { Surexploitation de } \\
\text { charbon de bois et } \\
\text { d'autres produits } \\
\text { forestiers non ligneux }\end{array}$ & $\begin{array}{l}\text { Probablement non décelables à partir de l'interprétation } \\
\text { des images satellite à moins d'un taux intensif de } \\
\text { importants dans la canopée } \\
\text { - Les approches basées sur les inventaires (enquêtes sur le } \\
\text { terrain) pourraient être mieux adaptées }\end{array}$ \\
\hline Extraction minière & $\begin{array}{l}\text { Difficile à surveiller en raison de la petitesse des clairières } \\
\text { qui les rendent quasiment indécelables }\end{array}$ \\
\hline
\end{tabular}

Source : Adapté de GOFC-GOLD (2008)

Les méthodes de surveillance basées sur la télédétection peuvent être appropriées quand la dégradation est suffisante pour laisser des trouées décelables dans la canopée comme c'est habituellement le cas pour l'abattage sélectif ou les feux de forêts. Il n'empêche que les mesures au sol sont des compléments importants, en particulier quand la dégradation ne cause pas de trouées dans la canopée comme dans le cas de bois mort et de végétation de sous-bois (Hardcastle et al. 2008).

On distingue habituellement deux approches principales de télédétection pour surveiller la dégradation des forêts (Achard et al. 2008) : une approche directe consistant à déceler les trouées dans les canopées des forêts et une approche indirecte qui repère les réseaux routiers et les aires de stockages de grumes.

- Approche directe surveillant l'abattage sélectif et les feux de forêts : Les méthodes basées sur cette approche sont axées sur la surveillance de la canopée des forêts pour y déceler des trouées ou des schémas correspondant à des processus de dégradation. ${ }^{2}$ Par exemple, Asner et al. (2005) ont conçu des algorithmes automatisés pour identifier des activités d'abattage en utilisant des données Landsat. Roy et al. (2005) ont mis au point une méthodologie pour cartographier des zones ravagées par des feux de forêts en utilisant des données MODIS. On a montré qu'il était possible d'obtenir un degré de précision de l'ordre de 86 à $95 \%$ dans l'interprétation de périmètres forestiers ayant subi un abattage sélectif ou ayant été brûlés (Achard et al. 2008).

\footnotetext{
Voir Achard et al. (2008) pour une description plus détaillée des méthodes relevant de cette catégorie.
} 
- Approche indirecte de surveillance de la dégradation des forêts : Dans cette approche, les terres forestières sont classées en " forêts intactes " (forêt non perturbée, à stock entier) et " forêts non-intactes » (forêt perturbée, à stock partiel, suite à l'extraction de bois ou à la dégradation de la canopée) sur la base d'une différenciation de critères combinant le couvert boisé (ou canopée) et l'impact anthropique qui peuvent être définis en fonction des circonstances nationales (Mollicone et al. 2007 ; Achard et al. 2008). ${ }^{3} \mathrm{La}$ dégradation des forêts est définie comme la conversion de forêts intactes en forêts non intactes.

\subsubsection{Estimation des stocks de carbone forestier}

Il est nécessaire d'estimer les stocks de carbone pour déterminer les émissions nettes liées aux forêts, ces estimations étant obtenues en combinant l'étendue de la superficie déboisée ou dégradée avec les mesures de densité du carbone. Les approches existant pour estimer les stocks de carbone forestier dans les pays tropicaux peuvent être regroupées en moyennes des biomes, en mesures au sol et en mesures de télédétection (Gibbs et al. 2007). Le Tableau 9.2 récapitule les bénéfices et les limitations propres à chaque méthode.

Des relations allométriques seront nécessaires pour convertir les inventaires forestiers et les données captées par les instruments de télédétection en mesures de carbone. Un certain nombre de relations existent au niveau mondial (par ex. Chave 2008), mais il est préférable d'élaborer des équations spécifiques aux pays dans ce domaine. Comme la plupart des pays dont les couverts forestiers sont denses ont des services de recherche forestière et que la création de relations allométriques est facile à réaliser, ils ne devraient pas avoir de difficultés à mettre au point les équations appropriées.

Il est souvent tentant d'utiliser les données des inventaires forestiers parce qu'un grand nombre de pays ont déjà dressé au moins un inventaire. Mais peu de pays en développement disposent d'inventaires nationaux exhaustifs et les données qui y figurent se rapportent souvent à des forêts de valeur commerciale seulement (DeFries et al. 2006).

\footnotetext{
3 Achard et al. (2008) suggèrent de définir la " forêt intacte " sur la base de six critères : 1) située dans une terre forestière conformément à la définition qu'en donne actuellement la CCNUCC, avec $1 \mathrm{~km}$ de zone tampon pénétrant dans la zone boisée, 2) une superficie supérieure à 1000 ha dont le plus petit côté doit être de $1 \mathrm{~km}, 3$ ) contenant une mosaïque continue d'écosystèmes naturels, 4) non fragmentée par l'infrastructure, 5) sans signes sensibles de transformation anthropique, et 6) sans terres brûlées ni sites de jeunes arbres et repousses adjacents aux projets d'infrastructure.
} 
Tableau 9.2. Bénéfices et limitations des méthodes existantes pour estimer les stocks de carbone forestier au niveau national

\begin{tabular}{|c|c|c|c|c|c|}
\hline \multirow{2}{*}{\multicolumn{2}{|c|}{$\begin{array}{l}\text { Méthode } \\
\text { Moyennes des } \\
\text { biomes }\end{array}$}} & Description & Bénéfices & Limitations & Incertitude \\
\hline & & $\begin{array}{l}\text { Estimations des stocks } \\
\text { moyens de carbone } \\
\text { forestier par grande } \\
\text { catégorie de forêt, sur } \\
\text { la base d'une série } \\
\text { d'intrants de diverses } \\
\text { sources }\end{array}$ & $\begin{array}{l}\text { - immédiatement } \\
\text { disponible } \\
\text { - des données étoffées } \\
\text { peuvent améliorer la } \\
\text { précision } \\
\text { - globalement } \\
\text { cohérents }\end{array}$ & $\begin{array}{l}\text { - plutôt généralisées } \\
\text { - l'échantillonnage } \\
\text { des sources de } \\
\text { données ne donne } \\
\text { pas une description } \\
\text { adéquate des } \\
\text { grandes superficies }\end{array}$ & Elevée \\
\hline \multicolumn{2}{|c|}{$\begin{array}{l}\text { Inventaires } \\
\text { forestiers }\end{array}$} & $\begin{array}{l}\text { Relient les mesures au } \\
\text { sol des diamètres des } \\
\text { troncs ou des volumes } \\
\text { des arbres aux stocks } \\
\text { de carbone forestier en } \\
\text { utilisant des relations } \\
\text { allométriques }\end{array}$ & $\begin{array}{l}\text { - relations génériques } \\
\text { facilement accessibles } \\
\text { - méthode peu } \\
\text { complexe facilement } \\
\text { compréhensible } \\
\text { - peut être relativement } \\
\text { peu coûteux, le facteur } \\
\text { le plus onéreux étant } \\
\text { la main d'œuvre sur le } \\
\text { terrain }\end{array}$ & $\begin{array}{l}\text { - les relations } \\
\text { génériques ne sont } \\
\text { pas adaptées à } \\
\text { toutes les régions } \\
\text { - peut être lent } \\
\text { - la nécessité } \\
\text { de produire } \\
\text { des résultats } \\
\text { globalement } \\
\text { cohérents constitue } \\
\text { un défi }\end{array}$ & Faible \\
\hline \multirow{2}{*}{ 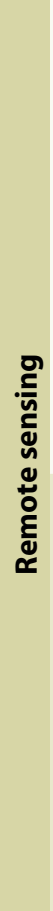 } & $\begin{array}{l}\text { Télédétecteurs } \\
\text { optiques }\end{array}$ & $\begin{array}{l}\text { Utilise fréquences } \\
\text { visibles et infrarouges } \\
\text { pour mesurer les } \\
\text { indices du spectre ; } \\
\text { est en corrélation avec } \\
\text { les mesures au sol de } \\
\text { carbone forestier (par } \\
\text { ex. Landsat, MODIS) }\end{array}$ & $\begin{array}{l}\text { - collecte régulière } \\
\text { de données } \\
\text { satellite librement } \\
\text { et globalement } \\
\text { accessibles } \\
\text { - globalement } \\
\text { cohérents }\end{array}$ & $\begin{array}{l}\text { - capacité limitée } \\
\text { d'élaborer de bons } \\
\text { modèles pour les } \\
\text { forêts tropicales } \\
\text { - saturation des } \\
\text { indices du spectre } \\
\text { quand les stocks de } \\
\text { carbone sont plutôt } \\
\text { bas } \\
\text { - peut être exigeant } \\
\text { sur le plan } \\
\text { technique }\end{array}$ & Elevée \\
\hline & $\begin{array}{l}\text { Télédétecteurs } \\
\text { optiques } \\
\text { aériens à } \\
\text { résolution fine }\end{array}$ & $\begin{array}{l}\text { Utilise des images à } \\
\text { résolution fine }(\sim 10 \text { à } \\
20 \mathrm{~cm}) \text { pour mesurer } \\
\text { la hauteur des arbres } \\
\text { et le houppier et } \\
\text { l'allométrie pour } \\
\text { estimer les stocks } \\
\text { de carbone (par ex. } \\
\text { photos aériennes, } \\
\text { imagerie aérienne } \\
\text { numérique en 3-D) }\end{array}$ & $\begin{array}{l}\text { - réduit le temps et } \\
\text { le coût de collecte } \\
\text { des données pour } \\
\text { dresser les inventaires } \\
\text { forestiers } \\
\text { - degré de précision } \\
\text { raisonnable } \\
\text { - excellente vérification } \\
\text { au sol pour le niveau } \\
\text { de référence de } \\
\text { déforestation }\end{array}$ & $\begin{array}{l}\text { - ne couvre que de } \\
\text { petites superficies } \\
\text { (10 000 ha) } \\
\text { - peut être onéreux et } \\
\text { complexe sur le plan } \\
\text { technique } \\
\text { - relations } \\
\text { allométriques } \\
\text { basées sur le } \\
\text { houppier non } \\
\text { accessibles }\end{array}$ & $\begin{array}{l}\text { Faible à } \\
\text { moyenne }\end{array}$ \\
\hline
\end{tabular}




\begin{tabular}{|c|c|c|c|c|c|}
\hline \multicolumn{2}{|c|}{ Méthode } & Description & Bénéfices & Limitations & Incertitude \\
\hline \multirow{2}{*}{ 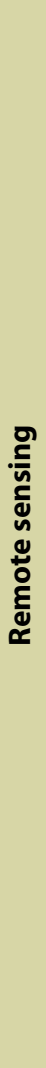 } & $\begin{array}{l}\text { Télédétecteurs } \\
\text { radar }\end{array}$ & $\begin{array}{l}\text { Utilise micro-ondes } \\
\text { ou signal radar pour } \\
\text { mesurer la structure } \\
\text { verticale de la forêt } \\
\text { (par ex. ALOS PALSAR, } \\
\text { ERS-1, JERS-1, Envisat) }\end{array}$ & $\begin{array}{l}\text { - données satellite } \\
\text { généralement libres } \\
\text { d'accès et gratuites } \\
\text { - nouveaux systèmes } \\
\text { lancés en } 2005 \\
\text { devraient fournir des } \\
\text { données améliorées } \\
\text { - peuvent être précis } \\
\text { pour les jeune } \\
\text { forêts ou les forêts } \\
\text { clairsemées }\end{array}$ & $\begin{array}{l}\text { - moins précis pour } \\
\text { les canopées } \\
\text { complexes de forêts } \\
\text { arrivées à maturité } \\
\text { parce que le signal } \\
\text { sature } \\
\text { - un terrain accidenté } \\
\text { (montagne) accroît } \\
\text { aussi le risque } \\
\text { d'erreur } \\
\text { - peut être coûteux et } \\
\text { complexe sur le plan } \\
\text { technique }\end{array}$ & Moyenne \\
\hline & $\begin{array}{l}\text { Télédétecteur } \\
\text { Laser (par ex. } \\
\text { Lidar) }\end{array}$ & $\begin{array}{l}\text { Lidar utilise la lumière } \\
\text { laser pour estimer la } \\
\text { hauteur et la structure } \\
\text { verticale de la forêt } \\
\text { (par ex. le système } \\
\text { satellite Carbon 3-D } \\
\text { combine la Vegetation } \\
\text { canopy Lidar (VCL) } \\
\text { avec l'imagerie } \\
\text { horizontale) }\end{array}$ & $\begin{array}{l}\text { - estime avec exactitude } \\
\text { la variabilité spatiale } \\
\text { complète des stocks } \\
\text { de carbone forestier } \\
\text { - le système satellite a } \\
\text { le potentiel suffisant } \\
\text { pour estimer les stocks } \\
\text { globaux de carbone } \\
\text { forestier }\end{array}$ & $\begin{array}{l}\text { - la seule option est } \\
\text { celle des capteurs } \\
\text { embarqués à bord } \\
\text { des aéronefs } \\
\text { - } \text { pas encore de } \\
\text { financement pour le } \\
\text { système satellite } \\
\text { - nombreuses } \\
\text { données à recueillir } \\
\text { sur le terrain à des } \\
\text { fins de calibrage } \\
\text { - peut être coûteux et } \\
\text { complexe sur le plan } \\
\text { technique }\end{array}$ & $\begin{array}{l}\text { Faible à } \\
\text { moyenne }\end{array}$ \\
\hline
\end{tabular}

\subsection{Estimation des émissions liées à la déforestation et à la dégradation des forêts}

Il est possible d'estimer les émissions nettes résultant des variations du paysage forestier en combinant les mesures qui portent sur les variations des superficies forestières avec celles qui évaluent les densités de carbone forestier. La quantité d'émissions libérées dans l'atmosphère résultant d'un changement d'affectation des terres dépendra non seulement du type de forêt mais aussi du changement spécifique. Par exemple, la conversion d'une forêt tropicale en champ de soja, de maïs ou de riz produit potentiellement $60 \%$ d'émissions en plus qu'une conversion en plantation de palmiers à huile (Miles et al. 2008). 


\subsubsection{Approches à travers les inventaires}

La méthode actualisée de comptabilisation des GES recommandée par le GIEC (GIEC 2006) inclut deux approches pour estimer les variations des stocks de carbone (Brown et Braatz 2008 ; Figure 9.1) : (i) l'approche basée sur les stocks ou approche des différences de stocks; et (ii) l'approche basée sur les processus ou approche des entrées et sorties.

1) Approche des différences de stocks

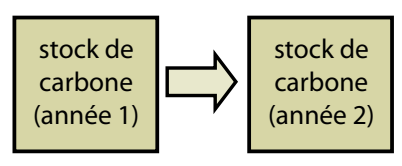

$\Delta \mathrm{C}=\left(\mathrm{C}_{\mathrm{t} 2}-\mathrm{C}_{\mathrm{t} 1}\right) /\left(\mathrm{t}_{2}-\mathrm{t}_{1}\right)$

Où

$\Delta C=$ variation annuelle de carbone dans le réservoir (tC/an)

$\Delta C_{t 1}=$ stock de carbone dans le réservoir au temps $\mathrm{t}_{1}(\mathrm{tC})$

$\Delta \mathrm{C}_{\mathrm{t} 2}=$ stock de carbone dans le réservoir au temps $\mathrm{t}_{2}(\mathrm{tC})$
2) Approche entrées-sorties

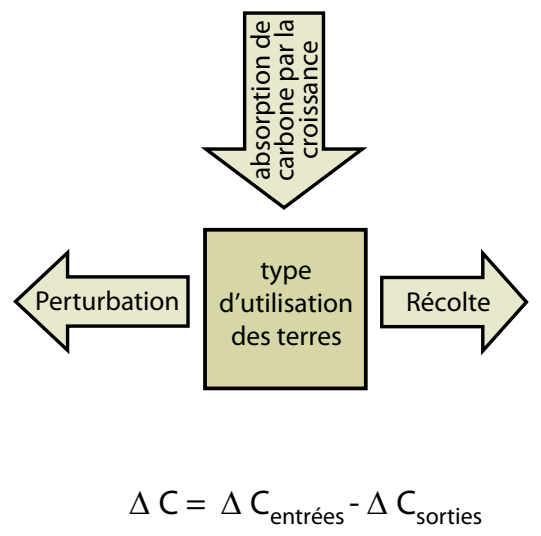

Où

$\Delta \mathrm{C} \quad=$ variation annuelle du stock de carbone dans le réservoir ( $\mathrm{tC} / \mathrm{an}$ )

$\Delta \mathrm{C}_{\text {entrèes }}=$ entrèes annuel de carbone (tC/an)

$\Delta \mathrm{C}_{\text {sorties }}=$ sorties annuelle de carbone (tC/an)

Figure 9.1. Estimation des variations des stocks de carbone (Wertz-Kanounnikoff 2008, adapté de Eggleston 2008, Brown et Braatz 2008)

- Approche des différences de stocks : Cette méthode estime la différence existant entre les stocks de carbone accumulés dans un réservoir donné à deux intervalles de temps distincts. Elle peut être utilisée quand les stocks de carbone dans les réservoirs concernés ont été mesurés et estimés dans la durée, comme dans le cas, par exemple, des inventaires forestiers nationaux. Cette approche convient parfaitement pour estimer les émissions causées à la fois par la déforestation et la dégradation et elle peut être appliquée à tous les réservoirs de carbone.

- Approche des gains et pertes : Cette approche estime le solde net des opérations d'additions et de soustractions effectuées dans un réservoir de 
carbone. Dans le contexte de la REDD, selon la façon dont est traitée la réhabilitation de l'écosystème, les gains résulteront de l'augmentation et du transfert de carbone d'un réservoir à l'autre (par ex. d'un réservoir de biomasse à un réservoir de matière morte organique, suite à une perturbation). Les pertes résultent donc d'un transfert de carbone vers un autre bassin et d'émissions dues à l'exploitation (l'abattage), à la décomposition ou aux feux de forêts. ${ }^{4}$ C'est la méthode utilisée quand on dispose de données annuelles sur des informations comme les taux de croissance et d'exploitation des forêts. En réalité, il est aussi possible de combiner les deux approches et de les utiliser toutes les deux.

\subsubsection{Complexité des inventaires}

Les méthodes décrites par le GIEC aboutissent à dresser des inventaires dont les niveaux de complexité différents sont classés par degrés ou niveaux (Tiers). En général, plus le degré de complexité des inventaires est élevé, plus la précision de leur contenu s'améliore et moins grande en sera l'incertitude. Le revers de la médaille est cependant que croissent aussi la complexité et les ressources requises pour dresser les inventaires correspondant aux niveaux supérieurs. Il est aussi possible de combiner des niveaux différents, par ex. le niveau 2 peut être utilisé pour la biomasse et le niveau 1 pour le carbone du sol en fonction des données disponibles et de l'ampleur des variations de stocks prévues dans le bassin.

Les méthodes correspondant au niveau 1 sont relativement simples à utiliser. Les lignes directrices du GIEC prévoient des équations et des valeurs de paramètres par défaut (par ex., facteurs de variations d'émissions et de stocks), de sorte que la personne qui compile les inventaires (le compilateur) n'aura pas besoin de données spécifiques pour ces éléments des équations. Des données spécifiques par pays sur l'utilisation des terres et la gestion sont nécessaires - ce qui ne constituera pas un problème puisque ces sources sont souvent facilement accessibles dès le premier niveau de complexité des inventaires (par ex. taux de déboisement, statistiques sur la production agricole, cartographie complète du couvert végétal terrestre, utilisation des engrais, données sur le cheptel). Une méthode qui ne se fonderait que sur ce premier degré de complexité ne devrait toutefois pas suffire à comptabiliser les crédits à attribuer au titre de la REDD.

La méthode du deuxième degré de complexité, le niveau 2, utilise la même approche méthodologique que celle du premier niveau, mais les facteurs de variation des émissions et des stocks de carbone sont basés sur des données spécifiques à l'échelle nationale ou régionale. Les facteurs d'émissions définis pour les pays conviennent mieux aux régions climatiques et aux systèmes

\footnotetext{
4 Quand les arbres sont abattus, il existe trois destinations possibles pour le carbone stocké : le bois mort, les produits ligneux et l'atmosphère (Pearson et al. 2008).
} 
d'utilisation des terres du pays ou de la région. Une résolution temporelle et spatiale plus élevée ainsi que des catégories de gestion et d'utilisation des terres plus désagrégées sont utilisées dans cette méthode et correspondent à des coefficients définis par les pays pour des régions spécifiques et des catégories d'utilisation des terres spécialisées.

S'agissant du troisième degré de complexité, le niveau 3, des méthodes plus complexes sont utilisées, y compris des modèles et des systèmes de mesure conçus pour traiter des circonstances nationales très spécifiques. Basées sur des données d'utilisation et de gestion des terres à haute résolution qui sont généralement désagrégées au niveau sous-national, les évaluations sont répétées dans le temps. Des techniques de mesure et/ou de simulation très avancées sont aussi utilisées pour obtenir de meilleures estimations des émissions et absorptions de GES - et des résultats bien supérieurs à ceux des deux méthodes précédentes.

\subsection{Intégration des méthodes MRV dans un mécanisme REDD}

Si les aspects techniques de la comptabilisation du carbone ont fait l'objet de progrès substantiels, de nombreux pays en développement n'ont encore pas suffisamment accès aux données et n'ont pas non plus l'infrastructure et les moyens techniques suffisants pour pouvoir analyser et gérer les données de façon cohérente et transparente. En outre, des institutions et des inventaires forestiers sont indispensables pour pouvoir effectuer les mesures sur le terrain, le contrôle de qualité et la vérification externe requis au titre des capacités MRV spécifiées pour la REDD.

Pendant les premiers stades de la mise en œuvre du mécanisme REDD, la plupart des pays auront probablement recours à la méthode des différences de stocks. A mesure que leurs capacités se renforceront, ils pourraient toutefois gagner en efficacité avec des approches basées sur les émissions (gains-pertes) qui permettent de mesurer directement les variations nettes d'émissions. Il est aussi très probable qu'un grand nombre de pays ne pourront utiliser qu'un système de comptabilité relevant du niveau 1 aux premiers stades de la mise en ouvre de la REDD et que ce sont donc des estimations basses des réductions d'émissions qui devraient être utilisées pour la comptabilisation des crédits (Eliasch 2008).

A la $15^{\mathrm{e}}$ Session de la Conférence des Parties (COP 15) à Copenhague en 2009, la communauté mondiale pourrait ne convenir pour commencer que d'un premier cycle de mise en œuvre de la REDD et fixer les responsabilités des pays en développement (Stern 2008). Dix années encore pourraient être nécessaires 
ensuite pour mettre en place des institutions de coopération efficaces ainsi que les capacités techniques et nationales nécessaires pour assurer et rentabiliser le suivi du programme et effectuer des mesures à diverses échelles (du local au national). Pendant cette phase, les pays ayant des capacités insuffisantes pour mettre en ouvre un système de comptabilisation correspondant aux degrés de complexité supérieurs pourraient participer à la REDD en utilisant la méthode de niveau 1 et en l'associant à des estimations basses de crédits. Les pays ayant les moyens de dresser des inventaires correspondant aux deuxième et troisième degrés de complexité - les niveaux 2 et 3 - sont aussi invités à contribuer au relèvement du niveau technique des autres participants. Reste toutefois l'incertitude de la trajectoire ultime de la REDD et de la façon dont ce mécanisme sera intégré dans les futurs agendas climatiques. Si la REDD est intégrée dans les marchés du carbone, il lui faudra gravir des échelons pour atteindre les niveaux de précision comptable requis par les acheteurs internationaux qui veulent être sûrs que des réductions d'émissions effectives ont bien eu lieu. D’un point de vue politique, un objectif pourrait être de créer un environnement propice à l'adoption progressive de méthodes comptables plus précises et moins incertaines relevant des degrés de complexité supérieurs. Cette évolution est cruciale pour les pays actuellement dotés de capacités MRV insuffisantes afin de les prémunir contre le risque d'être exclus d'un mécanisme complexe tout en leur donnant la possibilité d'améliorer leurs méthodes et leurs structures.

La création d'une institution internationale indépendante de surveillance du carbone forestier dans le cadre de la REDD ou l'inclusion d'un service de ce type dans une organisation existante pourrait être une autre façon de surmonter l'insuffisance de moyens techniques. Cette institution ne supplanterait pas le cadre établi par la CCNUCC pour les capacités MRV mais développerait les synergies nécessaires aux activités de surveillance de la REDD. Les pays de la Commission des forêts de l'Afrique centrale (Central African Forest Commission), par exemple, mettent en place une institution régionale, la Central African Forest Watchdog. La surveillance doit être d'autant plus précise, objective et fiable qu'elle débouche sur l'attribution de crédits carbone. Laisser à chaque pays participant à la REDD le soin d'assumer une telle tâche pourrait ouvrir la voie à des appréciations partiales (exagérant, par exemple, les réductions d'émissions effectives) pour tirer profit du marché du carbone. Un système de validation externe permet de contrôler les abus mais ajoute des coûts de transaction. Un meilleur choix pourrait donc bien être celui de confier la surveillance et la certification à un tiers indépendant, sous forme d'une institution internationale de surveillance du carbone forestier. Des économies d'échelle peuvent résulter de la centralisation de ces activités au niveau mondial, et en améliorer l'efficacité - par rapport à une situation où la recherche d'un suivi cohérent passe par chaque pays participant - sans oublier l'avantage d'une plus grande cohérence des données chronologiques sur la déforestation pour servir à l'établissement des niveaux de référence. 
Certaines estimations indiquent qu'un partenariat régional de surveillance entre le Cameroun, la République démocratique du Congo, la République du Congo, la Guinée Equatoriale et le Gabon en Afrique Centrale permettrait d'économiser plus de 2,2 millions de dollars US de frais d'établissement la première année et plus de 0,5 millions de dollars US par an de frais de fonctionnement ensuite (Hardcastle et al. 2008).

Par capacité on n'entend pas seulement l'existence d'équipement technique ou d'imagerie satellite coûteuse mais aussi - ce qui est souvent plus important le savoir faire qui est la somme des compétences acquises dans des spécialités comme le tri, le traitement et l'analyse des données, sans oublier l'utilisation de ces données dans le processus politique. Ce dernier point montre l'importance qu'il convient d'accorder à un renforcement des capacités qui comporte non seulement des aspects techniques (c.-à-d. pour mener des activités de surveillance dans les forêts) mais aussi des aspects politiques et institutionnels. Les décideurs politiques, par exemple, doivent avoir un minimum de connaissances pour comprendre comment les variations du carbone forestier influenceront les arrangements conclus au niveau national dans le cadre de la REDD et quels en seront les effets sur les autres politiques sectorielles.

La surveillance des émissions dues à la déforestation et à la dégradation des forêts est en butte à une autre difficulté qui tient au fait que nous n'avons qu'une connaissance partielle des stocks de carbone correspondant à d'autres types de forêts et utilisations forestières. C'est dans ce contexte que le Costa Rica, par exemple, a préconisé l'introduction d'un " principe de précaution » pour réduire le risque de surestimation (voir Appendice). Ce principe pourrait se traduire par des paiements effectués à l'extrémité inférieure de l'intervalle de confiance de $95 \%$. Bien qu'il existe des données par défaut et des lignes directrices du GIEC pour garantir l'utilisation d'estimations "basses », il reste encore à mieux inventorier dans l'espace les stocks de carbone forestier. Les capteurs Lidar sont particulièrement prometteurs à cet égard et devraient permettre de mesurer les futurs stocks de carbone forestier. Etant donné les besoins croissants de la REDD en matière de mesure et de surveillance et l'immense potentiel des capteurs Lidar (qui améliorent considérablement les estimations de biomasse), il serait judicieux d'envisager le déploiement d'une plateforme d'observation terrestre de ce type dans un proche avenir. De nouveaux investissements pourraient aussi cibler des activités comme la promotion de recherches opérationnelles pour une surveillance future de la biomasse au niveau mondial par des capteurs Lidar.

Les techniques d'imagerie à grande échelle par capteurs Lidar resteront d'accès limité au moins jusqu'en 2015-2017 et il faudra donc s'efforcer d'utiliser au maximum les autres moyens dont on dispose actuellement (par ex. les mesures au sol, les modèles des systèmes d'information géographique (SIG) pour extrapoler les données des échantillons). Au nombre des activités prioritaires 
devrait figurer l'établissement de relations allométriques pour différents types de forêts et de régimes de gestion. Lors d'une réunion récente d'un groupe d'experts de la CCNUCC sur les moyens de surveillance, de suivi et de contrôle $(\mathrm{MRV})$ à mettre en œuvre pour surveiller la dégradation, le groupe a reconnu la persistance d'importantes lacunes (en matière de données et de connaissances) et a recommandé d'utiliser les technologies et capacités MRV existantes le plus efficacement possible plutôt que de retarder les actions à engager en attendant des techniques améliorées (CCNUCC 2008b).

\subsection{Conclusion}

Nous avons cherché à démontrer que le système actuel que nous utilisons pour comptabiliser le carbone ne doit pas être un obstacle qui nous empêcherait d'inclure la REDD dans les agendas à venir du changement climatique. Dans ce résumé, nous avons présenté d'une part les progrès récemment accomplis par le GIEC dans l'élaboration de méthodes pour comptabiliser les GES et, d'autre part, les nouvelles avancées technologiques qui permettent d'améliorer la qualité des données utilisées dans ces méthodes. Nous en avons relevé les limitations mais aussi les possibilités existantes pour surmonter ces contraintes.

$\mathrm{Au}$ vu des récents progrès évoqués plus haut, nous pensons qu'il est possible de mettre en ouvre des systèmes de mesure et de validation viables pour la REDD. Nous savons que les situations des principaux pays forestiers sont inégales et qu'ils ne disposent pas tous des mêmes moyens pour mettre en œuvre ces systèmes. Une politique de l'environnement qui encourage l'innovation pour améliorer l'efficacité et apporte un soutien au renforcement des capacités contribuera à faire de la REDD un élément important de la lutte contre le changement climatique. Une approche progressive permettant de renforcer les capacités et de laisser les pays acquérir l'expérience nécessaire, avec l'intégration éventuelle du mécanisme REDD dans les projets de commercialisation des crédits carbone ou d'autres éléments appelés à faire partie d'un futur agenda climatique, assurera un caractère durable au processus de réduction des émissions.

Pour que le débat progresse sur les capacités MRV à inclure dans un mécanisme REDD, la $14^{\mathrm{e}}$ Session de la CCNUCC (COP-14) à Poznań devra préciser (i) comment la dégradation des forêts sera intégrée dans un futur programme de réduction REDD ; (ii) qui sera chargé de surveiller le processus de la REDD aux niveaux national et sous-national (s'agira-t-il d'une responsabilité assumée au niveau national ou au niveau international ?) ; et (iii) quelle sera la période ou l'année de référence pour déterminer les tendances historiques. Pour préparer le déroulement de ce futur processus de la REDD, les pays auraient tout intérêt à s'inspirer de règles et de lignes directrices claires telles que celles de la publication officielle du " guide des bonnes pratiques pour la REDD ». 



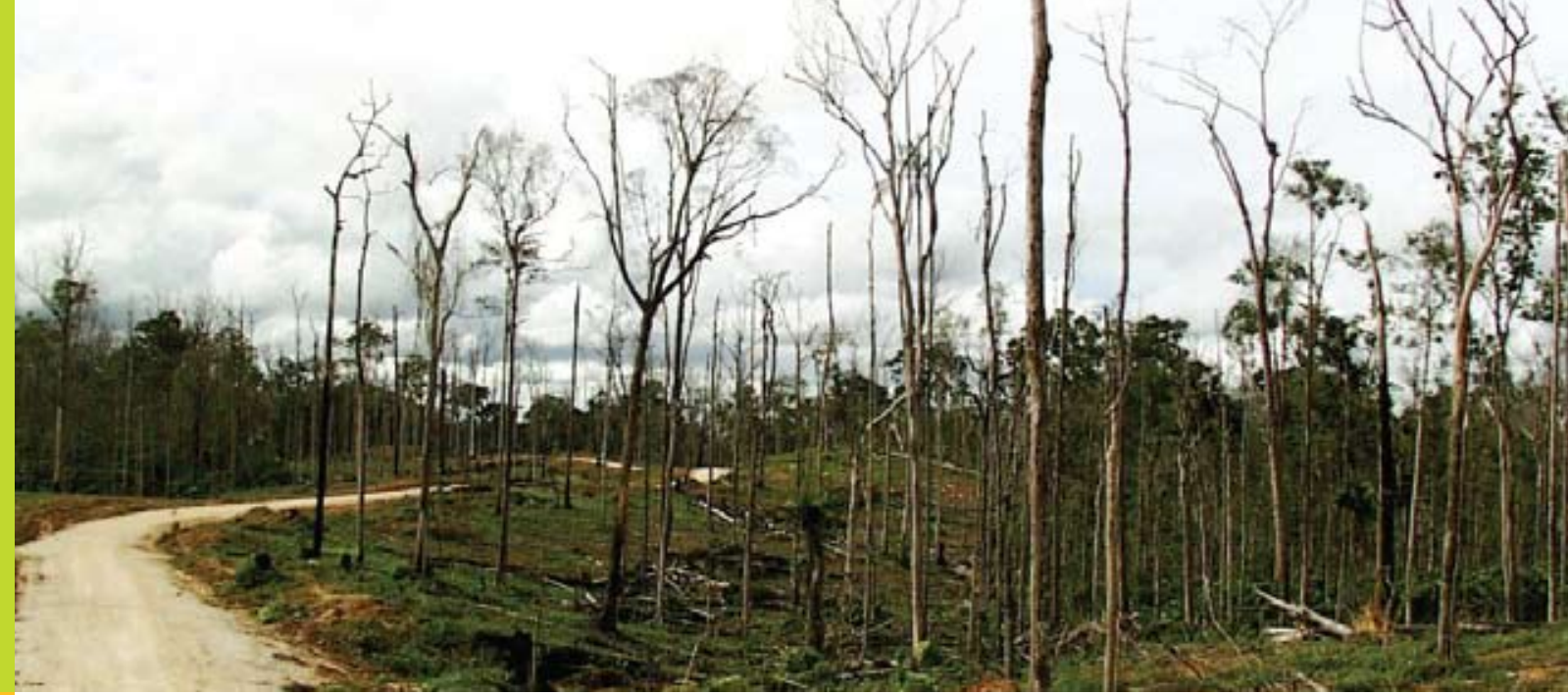

\section{Chapitire 10}

\section{Comment mesurer et surveiller la dégradation des forêts?}

Daniel Murdiyarso, Margaret Skutsch, Manuel Guariguata, Markku Kanninen, Cecilia Luttrell, Pita Verweij et Osvaldo Stella Martins

\subsection{Pourquoi la REDD comporte deux D}

La dégradation des forêts est une source importante de gaz à effet de serre (GES). Dans la forêt amazonienne brésilienne, la dégradation est responsable de $20 \%$ des quantités totales d'émissions (Asner et al. 2005). En Indonésie, le stock forestier diminue de $6 \%$ par an, les deux tiers de cette diminution étant attribuables à la dégradation tandis que la déforestation n'est responsable que du tiers restant (Marklund et Schoene 2006). En Afrique, le taux annuel de dégradation représente presque $50 \%$ du taux annuel de déforestation (Lambin et al. 2003).

En 2007, la treizième Conférence des Parties (COP 13) de la Convention des Nations Unies sur les changements climatiques (CCNUCC) a reconnu l'importance de la dégradation des forêts en l'incluant dans le mécanisme envisagé pour réduire les émissions dues à la déforestation et à la dégradation des forêts (REDD). Agir pour faire en sorte de diminuer la dégradation présente d'autres avantages importants au sens où des forêts moins dégradées seront mieux à même de s'adapter au changement climatique et de rendre davantage 
et de meilleurs services à l'écosystème et aux populations en améliorant leurs moyens d'existence.

Les facteurs déterminants de la déforestation d'une part, et de la dégradation des forêts d'autre part, diffèrent. En outre, la dégradation n'est pas nécessairement un agent précurseur de la déforestation. Les forêts peuvent rester dégradées pendant un très long laps de temps et ne jamais atteindre un stade de déboisement complet. Agir sur la déforestation ne réduit donc pas nécessairement les taux de dégradation des forêts. En outre, ne pas inclure la dégradation dans un accord sur la REDD signifierait que des quantités considérables d'émissions liées aux forêts ne seraient pas prises en compte. Si une forêt primaire saine, par exemple, dont le couvert boisé à $70 \%$ se dégrade jusqu'à ce que ce couvert ne soit plus que de $15 \%$, elle continuerait à être classée en tant que "forêt " et l'augmentation des émissions liées à la dégradation ne serait pas prise en compte.

Dans ce chapitre, nous avons mis l'accent sur les méthodes utilisées pour mesurer et surveiller la dégradation des forêts. Il complète et enrichit le chapitre 9 relatif aux deux D, la déforestation et la dégradation des forêts. Les méthodes mises au point pour mesurer et surveiller la dégradation des forêts sont étudiées du point de vue de leur efficacité à prendre en compte les émissions, de leur efficience par rapport aux coûts (ou rentabilité) et de leur équité sur le plan international. Les circonstances nationales qui sont différentes d'un pays à l'autre ont également été prises en compte.

\subsection{Définition et causes de la dégradation des forêts}

Adoptée à la neuvième session de la Conférence des Parties (COP 9) en 2003, la dégradation des forêts est définie comme " une perte directe à long terme d'origine humaine (persistant pendant $\mathrm{X}$ années ou davantage) d'au moins Y \% de stocks de carbone forestier (et de valeurs forestières) depuis un temps (T) et ne se qualifiant pas comme déforestation " (IPCC 2003a). Toutefois, la conclusion d'un accord sur une procédure opérationnelle pour surveiller, rendre compte et contrôler (Monitoring, Reporting and Verification, MRV) la dégradation n'a pas été sans mal (Penman 2008), la raison en étant qu'il est difficile de définir X (la perte à long terme d'origine humaine), Y (le pourcentage des stocks de carbone forestier) et le périmètre forestier minimum à mesurer. Chacun de ces facteurs est influencé par les activités qui sont à l'origine de la dégradation et par l'écologie de la forêt en cause. 
Quelques-unes des activités qui sont le plus couramment à l'origine de la dégradation des forêts sont notamment les suivantes (GOFC-GOLD 2008) :

- l'abattage sélectif;

- les feux de forêts à grande échelle ;

- l'extraction de produits forestiers non ligneux et de bois de chauffage ;

- la production de charbon de bois, le pâturage, les feux de sous-bois et les cultures itinérantes.

A l'exception de l'abattage sélectif, rares ont été les analyses qui ont été menées sur l'impact de ces activités sur la perte de biomasse forestière et sur le temps de régénération nécessaire pour les forêts. En outre, la plupart des études n'ont porté que sur les forêts tropicales humides. Il y a lieu de noter cependant que l'extraction de bois de chauffage dans les forêts sèches est à l'origine d'une détérioration bien plus poussée de la forêt que celle de l'exploitation commerciale du bois (Skutsch et Trines 2008). C'est un élément important puisque les forêts sèches sont habituellement plus densément peuplées que les forêts ombrophiles (rainforests). Alors que la teneur en carbone des forêts sèches est bien inférieure à celle des forêts humides, les forêts sèches représentent $42 \%$ des forêts tropicales (Murphy et Lugo 1986).

\subsection{Méthodes d'estimation des émissions liées à la dégradation des forêts}

Le GIEC (2003b) a identifié cinq bassins (ou réservoirs) de carbone qu'il conviendrait de mesurer (surveiller) afin d'estimer les émissions liées à la déforestation et à la dégradation des forêts : la biomasse aérienne, la biomasse souterraine, la litière, le bois mort et la matière organique du sol. La méthode la plus pratique pour estimer ces émissions consiste à ne mesurer que la biomasse aérienne. Toutefois, des processus de dégradation comme l'abattage et les feux ne peuvent qu'avoir un impact significatif sur les émissions émanant d'autres réservoirs de carbone comme la litière et le bois mort.

Le GIEC (2003b) prévoit aussi trois niveaux d'approche pour comptabiliser le carbone. Chaque niveau requiert un plus grand nombre de données et d'analyses complexes et devient donc plus précis :

- Le niveau 1 applique des facteurs d'émission par défaut (estimation des émissions sur la base des pertes de couvert forestier) aux données sur les activités forestières ("données sur les activités ») qui sont recueillies aux échelles nationale ou mondiale ; 
- le niveau 2 applique des facteurs d'émissions spécifiques aux pays et compile des données sur les activités ;

- le niveau 3 applique des méthodes, des modèles et des systèmes de mesure d'inventaires qui sont répétés dans le temps, pilotés par des données sur les activités à haute résolution et désagrégés au niveau sous-national à une échelle plus fine.

La surveillance, le suivi et la vérification des processus de déforestation et de dégradation comportent deux aspects : (i) la surveillance (et la mesure) des variations de superficie forestière par type de forêt; et (ii) la surveillance (et la mesure) des stocks moyens de carbone par unité de surface et par type de forêt (densités du carbone) (GIEC 2003b). Ainsi, dans l'approche la plus simple (niveau 1), on suit attentivement les variations de superficie boisée pour chaque catégorie de forêt et on calcule les stocks de carbone correspondant à chaque catégorie de forêt en utilisant des valeurs globales par défaut pour les densités de carbone. Dans l'approche de niveau 2, le degré de précision s'améliore parce que les densités de carbone sont estimées sur la base de données spécifiques aux pays et non plus sur des valeurs globales par défaut. Dans l'approche de niveau 3 , les modèles et les inventaires sont adaptés au pays concerné et répétés dans le temps ; les variations de densités du carbone sont là aussi mesurées dans le laps de temps correspondant à la période de comptabilisation.

Les variations de superficie forestière peuvent être mesurées grâce à la télédétection, au moins en partie, ou à des inventaires forestiers systématiques. Les inventaires doivent être basés sur des échantillons assez larges pour permettre de déceler des variations significatives de superficie forestière par type de forêt. Surveiller (et mesurer) la dégradation de la forêt (c.-à-d. le passage d'une forêt intacte à une forêt perturbée) par des techniques de télédétection est bien plus délicat que dans le cas de la déforestation. Il est facile, en effet, de repérer des périmètres déboisés grâce à la télédétection, en particulier quand la déforestation est pratiquée à grande échelle. Par contre, il est bien plus difficile de déceler des signes de dégradation parce que la télédétection ne permet pas de voir clairement, par exemple, que quelques arbres seulement ont été abattus (abattage sélectif) ou la perte de sous-bois (par le feu) ou la disparition de branches et de petits arbres (pour en tirer du bois de chauffage). Ces activités semblent n'avoir que peu d'effet sur le couvert forestier mais peuvent avoir un impact substantiel sur le stock de carbone forestier (DeFries et al. 2007). Même avec les techniques d'imagerie optique à haute résolution, il est difficile de déceler les changements sous le couvert arboré : des méthodes techniquement plus avancées comme le radar ont ce potentiel mais ne sont encore actuellement disponibles que dans quelques zones limitées.

Une façon de surmonter cette difficulté est d'adopter une approche probabiliste. Cela implique de stratifier la forêt en fonction des risques de dégradation, 
sur la base de tendances précédentes et de variables d'approximation comme l'accessibilité (par ex., densité des routes, distance par rapport aux établissements humains) (Schelhas et Sanchez-Azofeifa 2006). Les paramètres dans les modèles differeront en fonction des différents types de dégradation (par ex., abattage sélectif, collecte de bois de chauffage) (Iskandar et al. 2006).

Les variations des moyennes des stocks de carbone par unité de surface et par type de forêt peuvent être mesurées par plusieurs méthodes. Ces méthodes incluent l'utilisation de bases de données secondaires et d'estimations du GIEC (2003b), ainsi que l'élaboration in situ d'inventaires forestiers et la surveillance de parcelles-échantillons. Pour mesurer les variations des stocks de carbone causées par la dégradation des forêts, le GIEC (2006) recommande deux méthodes : la méthode de différence des stocks et la méthode de gains et pertes (voir Figure 9.1).

La méthode de différence des stocks s'appuie sur les inventaires forestiers traditionnels pour estimer la séquestration ou les émissions. La méthode des gains et pertes se construit à partir d'une réflexion sur l'écologie des forêts pour la comprendre : comment se déroule le processus de croissance des forêts, et comment les processus naturels ou anthropiques produisent des pertes de carbone. Dans la méthode de différence des stocks, ce sont les stocks réels de biomasse qui sont mesurés dans le réservoir (bassin) de carbone au début et à la fin de la période de comptabilisation. Dans la méthode des gains et pertes, ce sont les gains de la biomasse qui sont estimés en fonction des accroissements annuels moyens de la biomasse moins les pertes estimées de biomasse imputables à des activités d'exploitation du bois, d'abattage, de collecte de bois de chauffage et de surpâturage, sans oublier les feux. Si la forêt est stratifiée en zones soumises à divers processus de dégradation et si ceux-ci sont bien compris, il devrait alors être possible, par exemple, d'estimer avec une précision raisonnable les quantités de produits ligneux extraites pendant une période donnée.

Le Tableau 10.1 établit une comparaison entre la méthode de différence des stocks et la méthode de gains et de pertes. Les deux méthodes pourraient être utilisées pour évaluer la dégradation dans les approches de niveau 2 et 3 du GIEC. Le choix de la méthode dépendra largement du type de données disponibles et des ressources nécessaires pour recueillir des données supplémentaires (GOFCGOLD 2008). Les pays qui subissent une dégradation significative de leurs forêts pourraient souhaiter élaborer leurs propres bases de données et modèles nationaux spécifiques et utiliser la méthode des gains et pertes pour estimer les variations de leurs différents bassins de carbone. D'après les estimations de Hardcastle et Baird (2008), il semblerait qu'en ajoutant la dégradation aux facteurs entrant dans le calcul de l'approche de niveau 3, les augmentations de coût en résultant seraient de $10 \%$ de plus pour la République démocratique 
Tableau 10.1. Comparaison entre la méthode des différences de stocks et la méthode des gains et pertes pour estimer les émissions causées par différents types de dégradation

\begin{tabular}{|c|c|c|}
\hline $\begin{array}{l}\text { Type de } \\
\text { dégradation }\end{array}$ & Méthode des différences de stocks & $\begin{array}{l}\text { Méthode des gains et } \\
\text { pertes }\end{array}$ \\
\hline $\begin{array}{l}\text { Abattage } \\
\text { sélectif }\end{array}$ & $\begin{array}{l}\text { - Dans un contexte d'exploitation } \\
\text { légale du bois, une condition } \\
\text { habituellement imposée est } \\
\text { de mesurer la biomasse après } \\
\text { l'abattage - ce qui garantirait donc } \\
\text { la disponibilité des données } \\
\text { - Des données supplémentaires } \\
\text { devront être collectées en cas } \\
\text { d'abattage illégal } \\
\text { - Des données sur des forêts non } \\
\text { perturbées peuvent être utilisées } \\
\text { comme approximation si des } \\
\text { données antérieures aux activités } \\
\text { d'extraction du bois ne sont pas } \\
\text { disponibles pour un site particulier }\end{array}$ & $\begin{array}{l}\text { - Utilise des estimations des } \\
\text { accroissements annuels } \\
\text { moyens et des relevés } \\
\text { centralisés des activités } \\
\text { d'extraction du bois } \\
\text { - La fiabilité dépend de } \\
\text { l'honnêteté des sociétés } \\
\text { d'exploitation du bois } \\
\text { quand elles signalent les } \\
\text { taux d'extraction dans } \\
\text { leurs rapports }\end{array}$ \\
\hline $\begin{array}{l}\text { Feux de forêt à } \\
\text { grande échelle }\end{array}$ & $\begin{array}{l}\text { Les données de référence sur les } \\
\text { forêts non perturbées peuvent } \\
\text { être utilisées pour estimer la } \\
\text { biomasse en place avant un feu de } \\
\text { forêt, mais un inventaire forestier } \\
\text { sera nécessaire pour mesurer } \\
\text { la biomasse après la survenue } \\
\text { d'un feu }\end{array}$ & $\begin{array}{l}\text { - Les pertes dues au feu } \\
\text { peuvent être estimées } \\
\text { sur la base du périmètre } \\
\text { qui a brûlé. Les facteurs } \\
\text { d'émissions peuvent être } \\
\text { utilisés pour estimer les } \\
\text { émissions sur la base de la } \\
\text { biomasse perdue }\end{array}$ \\
\hline $\begin{array}{l}\text { Récolte de } \\
\text { bois de feu et } \\
\text { de produits } \\
\text { forestiers non } \\
\text { ligneux }\end{array}$ & $\begin{array}{l}\text { - Les niveaux de la biomasse } \\
\text { antérieurs à l'extraction du bois } \\
\text { pourraient être estimés sur la } \\
\text { base des niveaux types des } \\
\text { forêts non perturbées. Dans la } \\
\text { pratique, cependant, de grandes } \\
\text { portions de forêts soumises à ces } \\
\text { utilisations sont déjà en partie } \\
\text { dégradées au début de la période } \\
\text { de comptage } \\
\text { - Dans les zones déjà gérées par des } \\
\text { propriétaires individuels ou des } \\
\text { communautés, des inventaires } \\
\text { forestiers antérieurs et postérieurs } \\
\text { à ces activités pourraient être } \\
\text { dressés par les utilisateurs de } \\
\text { la forêt }\end{array}$ & $\begin{array}{l}\text { - Des données concernant } \\
\text { les pertes, par ex. des } \\
\text { registres sur les produits } \\
\text { commerciaux ligneux, des } \\
\text { estimations sur l'utilisation } \\
\text { de bois de chauffage, } \\
\text { pourraient être disponibles } \\
\text { - L'extraction de bois de } \\
\text { chauffage pourrait aussi } \\
\text { être calculée sur la base de } \\
\text { données démographiques } \\
\text { (population) et de données } \\
\text { sur la consommation } \\
\text { moyenne des ménages } \\
\text { (utilisation du bois de } \\
\text { chauffage par les familles) } \\
\text { - Les chiffres sur les gains } \\
\text { peuvent être extraits } \\
\text { des statistiques types } \\
\text { sur les accroissements } \\
\text { annuels moyens }\end{array}$ \\
\hline
\end{tabular}




\begin{tabular}{|c|c|c|}
\hline $\begin{array}{l}\text { Type de } \\
\text { dégradation }\end{array}$ & Méthode des différences de stocks & $\begin{array}{l}\text { Méthode des gains et } \\
\text { pertes }\end{array}$ \\
\hline $\begin{array}{l}\text { Feux de sous- } \\
\text { bois, pâturage } \\
\text { et cultures } \\
\text { itinérantes } \\
\text { (utilisation de } \\
\text { la forêt pour } \\
\text { la production } \\
\text { agricole) }\end{array}$ & $\begin{array}{l}\text { - Les niveaux de la biomasse } \\
\text { antérieurs à l'extraction du } \\
\text { bois pourraient être estimés } \\
\text { à partir des niveaux types des } \\
\text { forêts non perturbées. Mais la } \\
\text { plupart des forêts soumises à ces } \\
\text { changements d'affectation sont } \\
\text { déjà en partie dégradées au début } \\
\text { de la période de comptage } \\
\text { - Les communautés peuvent } \\
\text { mesurer les changements qui } \\
\text { surviennent et cela peut favoriser } \\
\text { le processus « d'appropriation » }\end{array}$ & $\begin{array}{l}\text { - Les chiffres sur les gains } \\
\text { peuvent être extraits } \\
\text { des statistiques types } \\
\text { sur les accroissements } \\
\text { annuels moyens } \\
\text { - Les données sur les pertes } \\
\text { sont rarement disponibles } \\
\text { dans les statistiques } \\
\text { nationales }\end{array}$ \\
\hline
\end{tabular}

du Congo, $11 \%$ pour l'Indonésie et $13 \%$ pour le Brésil. Les pourcentages d'accroissement des frais ordinaires récurrents seraient du même ordre de grandeur. Dans ces calculs cependant, l'hypothèse de départ est que ces pays rendent déjà des comptes dans le cadre de l'approche de niveau 3 et qu'ils ont donc déjà en place de robustes systèmes d'échantillonnage (recouvrant au moins $3 \%$ de leur surface terrestre et strates).

\subsection{Répercussions sur les coûts dans les différents pays}

Les coûts encourus au titre de la mesure et de la surveillance du processus de dégradation dans les forêts dépendent des circonstances propres à chaque pays, notamment :

- de l'étendue du couvert boisé ;

- du niveau de stratification forestière (par exemple, la République démocratique du Congo n'a qu'un seul grand type de forêt tandis que l'Indonésie et le Mexique ont quatre écotypes forestiers ou davantage) ;

- du niveau de comptabilisation appliqué pour le carbone.

Les forêts des différents pays ont atteint divers stades de leur courbe de transition (Figure 10.1), reflétant ainsi les changements d'affectation des terres (conversion en terres agricoles) et des rentes forestières au fil du temps (Angelsen 2007). Il en découle que la dégradation revêt des aspects plus critiques dans certains pays que dans d'autres. Il peut arriver, par exemple, que des pays aient cessé de déboiser mais continuent à perdre de la biomasse dans les zones forestières qui leur restent. Autrement dit, l'état (de santé) des forêts d'un pays aura une influence sur la mesure dans laquelle ce pays investira pour se doter de systèmes 


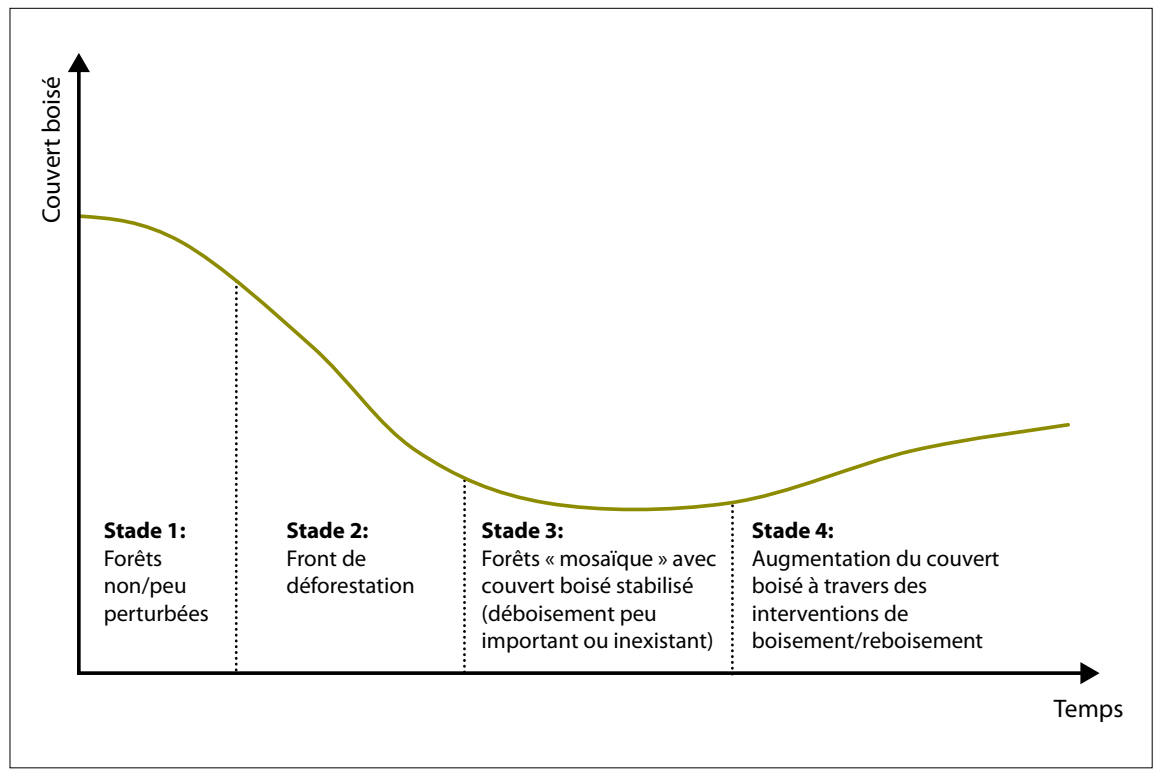

Figure 10.1. Stades de la transition des forêts (adapté de Angelsen 2007)

qui comptabiliseront la dégradation de ses forêts ainsi que sur les systèmes de mesure et de surveillance pour lesquels il optera.

La théorie de la transition des forêts identifie quatre stades de transition pour les forêts. On peut distinguer quatre grandes catégories de pays selon le stade dont relèvent leurs forêts :

1. Les pays et les régions caractérisés par un faible niveau de déboisement et un couvert forestier dense comme le Bassin du Congo et la Guyane Dans ces régions, les forêts sont relativement non perturbées mais peuvent être soumises à une déforestation et une dégradation croissantes à l'avenir. Ces pays et régions seront probablement les plus intéressés par la prise en compte de la dégradation des forêts parce que ce sont les moins susceptibles de bénéficier d'une "déforestation évitée ", du moins si les niveaux de référence sont fixés à partir de la déforestation historique. Dans ces pays qui gardent encore des forêts intactes, la méthode la plus rentable de comptabilisation du carbone sera sans doute celle des différences de stocks associée à un échantillonnage stratifié. Des approximations pourraient être utilisées au cas où il n'y aurait pas de données antérieures à l'abattage ou à d'autres interventions humaines (Tableau 10.1). Pour les pays qui ont accueilli de grandes concessions d'exploitation du bois, la méthode des gains et pertes pourrait être la plus efficiente sur le plan des coûts parce qu'ils disposeraient de toutes les données de base nécessaires au titre de l'approche de comptabilisation de niveau 2 pour appliquer cette approche. 
Ces pays pourraient être incités à prendre en compte la dégradation à travers un soutien financier qui leur serait accordé à cette fin.

2. Les pays caractérisés par une forte déforestation comme c'est le cas dans certaines parties du Brésil, de l'Indonésie et du Ghana avec de grands pans de forêts affectés de taux de déboisement élevés (front de déforestation) - Ces pays ont toutes les raisons de s'engager dans la voie de la comptabilisation du déboisement. Par contre, ils seront moins enclins à s'intéresser à une prise en compte de la dégradation de leurs forêts à moins que l'effort supplémentaire qui leur serait demandé pour ce faire soit peu important. Il y a lieu de noter toutefois qu'en excluant la dégradation des facteurs pris en compte dans les plans nationaux de la REDD (en particulier dans les régions où prévaut l'abattage sélectif), ces pays courent le risque de subir des fuites considérables. S'agissant de la méthode, il est vraisemblable qu'ils choisiront la méthode des gains et pertes pour les mêmes raisons que celles qui ont été données pour les pays de la catégorie 1 où prévalent de grandes concessions d'exploitation forestière.

3. Les pays caractérisés par une déforestation et un couvert forestier peu importants ainsi que par un paysage forestier de type "mosaïque " et des zones boisées stabilisées - Dans ces pays, les taux de déboisement se sont aplanis, soit parce que les forêts ont déjà été largement défrichées soit parce que les politiques de protection des forêts sont appliquées avec rigueur. L'Inde pourrait relever de cette catégorie et, ainsi qu'elle l'a indiqué dans sa soumission de 2008 à la CCNUCC, elle pourrait être intéressée par une réduction de la dégradation en combinant probablement cet objectif à ceux de la conservation de ses forêts, de boisement et de reboisement et d'autres options pour renforcer ses stocks de carbone forestier. Les pays faisant partie de cette catégorie pourraient utiliser la méthode des différences de stocks de niveau 2 et progresser à l'échelon supérieur, de niveau 3, à mesure d'un accès plus facile et rentable à des données spécifiques aux sites concernés.

4. Les pays dont le couvert forestier est en voie d'expansion, comme la Chine et le Vietnam - Ces pays pourraient n'être que modérément intéressés par la prise en compte de la dégradation des forêts à moins que l'accord sur la REDD intègre la notion du renforcement des stocks de carbone forestier (chapitre 2). Il y a lieu de noter toutefois que même si les nouvelles plantations augmentent la superficie forestière dans ces pays, les forêts existantes pourraient bien simultanément se détériorer. Ces pays pourraient choisir de présenter leur réussite -à savoir l'augmentation de leur superficie de plantations forestières - comme des activités de boisement et reboisement $(\mathrm{B} / \mathrm{R})$ menées au titre du mécanisme de développement propre (MDP). Qu'ils retiennent ou non cette option dépend de l'intégration ou non des activités de boisement/reboisement dans un futur accord sur la REDD. Comme ces pays pourraient avoir recensé leurs activités de gestion forestière depuis assez longtemps, ils pourraient aussi disposer de bases de données susceptibles de servir de scénarios de référence historiques qui leur permettraient d'adopter la méthode des différences de stocks de niveau 3. 


\subsection{Conclusion}

Plus complexe, la dégradation des forêts est plus difficile à définir, mesurer, surveiller et vérifier que la déforestation (GIEC 2003a). Il convient donc d'utiliser un plus grand nombre de facteurs d'approximation. Les méthodologies des différences de stocks, des gains-pertes (IPCC 2006) et d'approches graduelles à trois niveaux (IPCC 2003b), recommandées par le GIEC, sont utiles pour rendre compte du rôle du carbone dans la dégradation des forêts. Quand les données sont peu nombreuses, on peut avoir recours à des méthodes simples, des valeurs par défaut (approche de niveau 1) et des approximations pour mesurer les émissions causées par divers types de dégradation. Les incertitudes inhérentes à ces approches plus simples impliquent qu'il sera nécessaire « d'actualiser » les crédits - ce qui constituera une incitation directe pour les pays et les encouragera à améliorer leurs méthodes de mesure et de surveillance.

S’il est possible de relever les défis liés à la prise en compte du carbone dans la dégradation des forêts grâce aux deux méthodes préconisées par le GIEC - les différences de stocks et les gains-pertes - et à l'approche graduelle à 3 niveaux, il semble aussi réaliste d'inclure la dégradation dans un accord sur la REDD. Il en résulterait une plus grande efficacité de ce mécanisme puisqu'un plus grand nombre de catégories d'émissions de gaz à effet de serre liées aux forêts serait comptabilisé. Il en découlerait aussi une plus grande équité à l'échelle internationale parce qu'un plus grand nombre de pays - dont de nombreux pays du continent africain - seraient encouragés à participer. Il importe donc que les décisions relatives aux mesures de surveillance, de suivi et de vérification de la dégradation tiennent compte de la diversité des circonstances. L'objectif est réalisable à condition de laisser suffisamment de flexibilité aux pays pour qu'ils élaborent et appliquent des méthodes de comptabilisation du carbone rapportées à la dégradation de leurs forêts. 


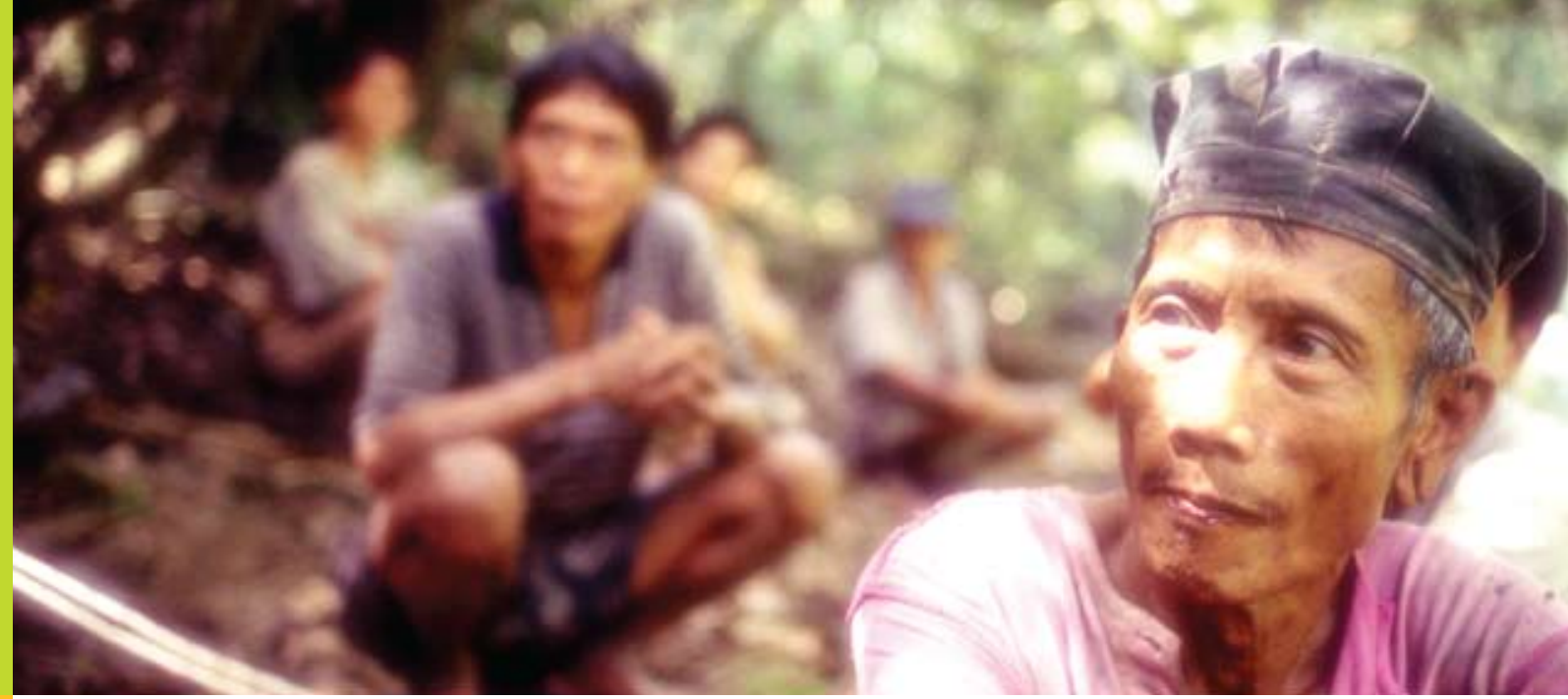

\section{Chapitire 11}

\section{Comment tirer parti des bénéfices associés à la REDD et éviter les effets nocifs?}

David Brown, Frances Seymour et Leo Peskett ${ }^{1}$

\subsection{Introduction}

Les négociations sur le changement climatique mondial ne concernent pas uniquement la réduction des émissions des gaz à effet de serre. L'Article 2 de la Convention-cadre des Nations Unies sur les changements climatiques (CCNUCC) spécifie que l'objectif ultime de la convention est de stabiliser les concentrations de gaz à effet de serre dans l'atmosphère tout en veillant à ce que la production alimentaire ne soit pas menacée et que le développement économique puisse se poursuivre d'une manière durable. Réunie en sa treizième session à Bali en décembre 2007, la Conférence des Parties (Décision 2/CP.13) a reconnu que la réduction des émissions résultant du déboisement et de la dégradation des forêts (REDD) « peut avoir des retombées positives et servir des buts et objectifs d'autres conventions et accords pertinents » et " qu'il faudrait prendre en compte les besoins des populations locales et des communautés autochtones dans le cadre de l'action engagée " pour mettre en œuvre la REDD.

Ce chapitre est tiré de Brown et Peskett (2008), Peskett et al. (2008) et Seymour (ouvrage en préparation). 
Les parties à la CCNUCC ont ainsi reconnu que la REDD aura des répercussions qui iront bien au-delà de l'atténuation des émissions de $\mathrm{CO}_{2}$. Dans ce chapitre, nous replaçons la REDD dans une perspective plus large et étudions plus particulièrement ses "retombées positives " (ou bénéfices associés), en mettant l'accent sur :

- les retombées positives sur le plan social (associées à un développement en faveur des pauvres) ;

- la protection des droits de l'homme et l'amélioration de la gouvernance forestière ; et

- les retombées positives pour l'environnement, en particulier le renforcement de la protection de la biodiversité et l'amélioration de la qualité des sols et de l'eau ainsi que de leur disponibilité.

Dans ce chapitre, nous examinons la mesure dans laquelle les diverses options et formules envisagées pour la REDD dans les chapitres précédents peuvent être rendues compatibles avec les retombées positives souhaitées et éviter les effets nocifs. En conséquence, pour chacune des trois grandes catégories de retombées positives envisagées, un résumé succinct a été établi pour en décrire :

- les opportunités et les défis qui relèvent directement des négociations sur l'architecture globale d'un accord sur la REDD ; et

- les répercussions sur la mise en œuvre de la REDD au niveau national et sous-national.

La REDD fait actuellement l'objet de négociations dans le contexte d'un certain nombre d'accords internationaux et instruments y afférents qui reconnaissent l'importance de la gestion des ressources forestières pour aboutir à des retombées positives sur le plan social. La " feuille de route de Bali » se réfère à ces instruments dans les " directives indicatives " pour les activités de démonstration qui « devraient être compatibles avec une gestion durable des forêts et tenir compte, notamment, des dispositions pertinentes du Forum de Nations Unies sur les forêts, de la Convention des Nations Unies sur la lutte contre la désertification et de la Convention sur la diversité biologique " (Décision 2/CP.13 - Annexe). Par exemple, l'Article 20 de la Convention sur la diversité biologique reconnaît que le développement économique et social et l'élimination de la pauvreté sont les priorités premières et absolues des pays en développement partenaires, et que l'aide internationale doit s'y adapter en conséquence. L'instrument juridiquement non contraignant du Forum des Nations Unies sur les forêts inclut dans ses objectifs celui de " renforcer la contribution des forêts à la réalisation des objectifs de développement internationalement reconnus, y compris les objectifs de développement $\mathrm{du}$ Millénaire, sur l'élimination de la pauvreté et l'environnement durable ... » 
(Paragraphe II, Principe 1). Tous ces accords - et des instruments tels que les politiques de sauvegarde des banques de développement multilatérales fournissent un ensemble émergent de textes sur des normes internationales applicables à la REDD.

En même temps, de solides arguments sont avancés pour que le mécanisme de la REDD reste simple afin de ne pas surcharger un programme déjà lourd et décourager les investissements en mettant trop l'accent sur les retombées positives et les mécanismes de sauvegarde. Comme dans le cas des autres options étudiées dans ce livre, il s'agira ici également de tenir compte des avantages et inconvénients potentiels de ces options du triple point de vue de l'efficacité, de l'efficience et de l'équité.

\subsection{Bénéfices associés à la réduction de la pauvreté et à l'amélioration de l'équité}

Toutes les questions visant à déterminer si et comment les retombées positives sur le plan social pourraient être incluses dans l'architecture de la REDD et ses prestations font l'objet de vifs débats. Il existe deux écoles de pensée chez ceux qui sont en faveur d'une inclusion de la REDD dans un agenda sur le changement climatique. Certains avancent que l'objectif principal de la REDD étant de s'attaquer au changement climatique et non à la pauvreté, la bonne façon de procéder serait de " ne pas porter préjudice " aux pauvres. ${ }^{2}$ D'autres qui sont partisans d'une approche en faveur des pauvres allèguent que la REDD ne peut réussir sans retombées positives effectives. Ce groupe estime que c'est de sa capacité à améliorer le bien-être des populations pauvres dépendantes des forêts et à promouvoir le développement de certaines des régions les plus pauvres du monde que la REDD tire une grande partie de sa légitimité et de son efficacité potentielle. Les arguments avancés en faveur d'une approche en faveur des pauvres sont très divers mais convaincants (voir Encadré 11.1).

La REDD pourrait bien se révéler à hauts risques pour les communautés pauvres dépendantes des forêts. La multiplicité des intérêts et la polarisation des richesses et du pouvoir des différentes parties prenantes dans le secteur forestier sont au nombre de ces risques. Cependant, la REDD offre aussi d'importantes opportunités pour réduire la pauvreté et renforcer l'équité grâce à un afflux substantiel de capitaux - dans les zones rurales, qui font partie des zones les plus démunies et les moins bien loties de la plupart des économies en développement.

\footnotetext{
2 Par exemple, une communication présentée à la CCNUCC en 2007 par le Gouvernement de Tuvalu relève que "...des retombées positives seraient possibles mais qu'elles ne devraient pas être plus importantes que le principe fondamental qui est de réduire les émissions au niveau du globe » (CCNUCC 2007).
} 


\section{Encadré 11.1. Pourquoi la REDD doit adopter une approche en faveur des pauvres}

Les arguments moraux concernent la nécessité non seulement de veiller à ce que toute initiative internationale importante vise à améliorer le bien-être et l'équité mais aussi à prendre en compte les intérêts de ceux qui ont des droits légitimes sur l'utilisation des ressources de la forêt et qui pourraient subir un préjudice du fait d'interventions acceptées par la communauté internationale.

Des considérations d'ordre pratique donnent à penser que ce sont ceux qui vivent à proximité immédiate de la forêt et en gèrent les ressources - et qui sont souvent les communautés pauvres dépendantes des forêts - qui auront besoin d'incitations appropriées pour garantir l'efficacité de la REDD.

Au nombre des arguments plaidant en faveur des moindres risques figurent le risque d'être rejeté par sa communauté, voire celui de conflit social - ce qui pourrait peser d'un poids considérable pour dissuader les investisseurs étrangers compte tenu, en particulier, des antécédents bien connus de la foresterie en tant qu'arène politique polémique.

Les investissements au titre de la REDD seront encore plus attrayants pour les investisseurs dont les motivations sont liées à la responsabilité sociale des entreprises si les prestations de la REDD incluent des retombées positives pour les pauvres.

Considérations d'ordre politique : Une grande partie des sources de financement de la REDD sera constituée d'institutions de développement et de bailleurs internationaux pour lesquels le développement social constitue le fondement même de leurs actions.

Justification par la procédure : La CCNUCC reconnaît l'importance des questions sociales, y compris la pauvreté, en tant que priorités mondiales (Décision 2/CP.13).

\subsubsection{Pertinence par rapport à l'architecture de la REDD à l'échelle mondiale}

Dans les précédents chapitres, l'incidence de divers éléments constitutifs de la REDD sur l'équité a été évaluée ainsi que les avantages et inconvénients du point de vue de l'efficacité et l'efficience (des coûts). Certains compromis possibles sont brièvement résumés ci-après :

\section{Un financement basé sur le marché ou provenant d'un fond internationale} (chapitre 5) : Les modèles de financement élaborés pour la REDD auront d'importantes répercussions sur la pauvreté et l'équité. Les différences les plus flagrantes devraient concerner les volumes de financement avec un afflux de capitaux beaucoup plus important en provenance des marchés réglementaires que le financement accordé sous conditions par un fonds international. Les systèmes basés sur le marché présentent cependant deux gros inconvénients. Tout d'abord, les marchés ne financeront probablement pas les services 
de la REDD liés à la production de biens publics, comme les mesures de préparation à la mise en œuvre de la REDD. Il y a tout lieu de craindre que le financement par les marchés se limite aux aspects politiquement les moins risqués (par exemple, le renforcement des moyens de mesure et de surveillance techniques) au détriment des grandes réformes politiques et institutionnelles qui pourraient aider la REDD à réaliser son potentiel de développement, par exemple les réformes du régime foncier.

En deuxième lieu, un financement basé sur le marché risque d'être inégalement réparti entre les économies émergentes (qui tendent à avoir des cadres juridiques et des marchés financiers très élaborés qui suscitent la confiance du secteur privé) et les pays moins avancés (qui tendent à être caractérisés par une "gouvernance médiocre »). Il est peu probable que les investisseurs choisissent d'investir dans des pays où la gouvernance est insuffisante et les capitaux ne devraient donc affluer que vers les économies émergentes comme cela a été le cas pour le mécanisme de développement propre (MDP) (Ebeling et Yasue 2008). Plus le pays et les groupes bénéficiaires potentiels de ce pays sont pauvres, plus s'amenuise la probabilité de capitaux effectifs versés d'avance pour financer des activités liées à la REDD.

Dans le court à moyen terme, des considérations liées à la gouvernance laissent entendre que la plus grande partie du financement accordé aux pays les moins avancés aura pour origine des sources d'aide volontaires et ne seront pas en provenance de marchés réglementaires même si, dans le cadre de l'approche combinée, certains projets peuvent être financés en dépit de situations nationales défavorables. En principe, le financement des bailleurs institutionnels sera davantage en faveur des pauvres que celui du marché financier réglementaire, notamment, parce que les institutions chefs de file seront mandatées pour promouvoir des agendas de développement. Une autre approche consiste à recourir à une imposition (par exemple, un pourcentage fixe du produit résultant de la mise aux enchères des permis d'émissions du système ETS de l'Union Européenne). Elle pourrait associer les avantages du marché financier (on estime qu'une redevance de $5 \%$ pourrait rapporter 2,5 milliards d'euros d'ici 2020) à ceux de retombées positives et présente donc un attrait certain (Euractiv 2008). S'agissant du financement basé sur l'apport de fonds institutionnels (qu'il s'agisse d'aide au développement ou de recettes fiscales dérivées d'un système de taxation), il convient de relever qu'il affaiblit le lien entre le paiement et la performance et qu'il risque de renouveler les mauvais résultats de l'aide traditionnelle apportée au secteur forestier.

Champ d'application et définitions de la forêt (chapitre 2): Le champ d'application de la REDD et les définitions données du terme " forêt " ont d'importantes répercussions sur la détermination des pays ou des groupes qualifiés pour bénéficier des flux financiers de la REDD. L'inclusion de la dégradation, par exemple, a des effets différents selon qu'il s'agit de pays où la déforestation est 
principalement due à la conversion industrielle massive des terres (par ex., le Brésil) ou de pays où la déforestation est causée de façon plus progressive par la conversion en terres agricoles (petites exploitations) et la demande de bois de chauffage et de charbon de bois (par ex., un grand nombre de pays d'Afrique). L'adoption d'une définition qui ajoute la dégradation à la déforestation élargit donc le champ d'application potentiel des mécanismes incitant les populations pauvres à conserver le carbone. Un impact potentiellement négatif pourrait être que les activités considérées comme dégradant le carbone (la culture itinérante, par exemple) pourraient être traitée de façon répressive. D’un autre côté, des définitions étroites pourraient absorber la plus grande partie des financements disponibles au détriment d'interventions en faveur des pauvres.

Risque et responsabilité (chapitre 8) : Les questions relatives aux risques et aux responsabilités sont au cœur des préoccupations des marchés réglementaires. Un grand nombre d'acheteurs internationaux seront motivés par le désir d'effectuer de gros volumes de transaction au moindre risque et les activités en faveur des pauvres pourraient en pâtir sur les deux fronts. Rendre les autorités nationales entièrement responsables des prestations pourrait entamer gravement leur bonne volonté et les dissuader d'investir dans des activités axées sur les pauvres. Les autorités nationales sont aussi moins enclines à répercuter les fonds qu'elles recevraient par avance à l'échelon des communautés rurales. Le principe de la responsabilité en aval (au cas où le projet en question ne génèrerait pas les réductions d'émissions promises) pourrait devenir un véritable casse-tête pour les acteurs et les communautés pauvres si leurs gouvernements, au nom des investisseurs, devaient les charger d'une telle responsabilité.

Echelle (chapitre 4) : L’architecture élaborée pour alimenter les activités liées à la REDD auront aussi un impact sur la qualité des réformes en faveur des pauvres, sans oublier quelques autres effets importants liés à l'échelle choisie. Par exemple, une approche combinée avec une responsabilité initialement encourue au niveau du projet favorisera des interventions qui seront caractérisées par les forces et les faiblesses propres à ce niveau. Si des paiements sont effectués et comptabilisés au niveau du projet, cela pourrait faciliter une gestion plus rigoureuse, mais il pourrait être difficile d'informer ou d'influencer le milieu politique à un échelon plus élevé de la hiérarchie - milieu qui est pourtant le plus susceptible d'avoir un impact sur les facteurs déterminants de la déforestation. Une approche qui privilégie les actions au niveau national et favorise un alignement des flux financiers sur les processus budgétaires nationaux et l'harmonisation avec les stratégies nationales en faveur des pauvres axées sur la réduction de la pauvreté sera mieux à même d'influencer le milieu politique même s'il se révèle plus vulnérable aux échecs de la gouvernance et à la corruption. 


\subsubsection{Opportunités et défis au niveau national}

Si l'architecture et le cadre de la mise en œuvre de la REDD sont fixés à l'échelle internationale, les retombées positives pour réduire la pauvreté et améliorer l'équité dépendront largement de la façon dont les paiements incitatifs de la REDD se traduiront en stratégies de réductions d'émissions au niveau national. Les politiques et mesures adoptées pourraient aller de politiques décidées à ce niveau (par exemple, élimination des subventions qui encouragent le déboisement et la dégradation des forêts, taxation des opérations de défrichement, planification stratégique de l'infrastructure routière), en passant par des pratiques industrielles améliorées (telles qu'un soutien apporté à la certification du bois et à la réduction de l'impact de l'abattage), à des initiatives ayant une incidence directe sur les moyens d'existence des populations pauvres (programmes pour des modes de vie alternatifs, stratégies de prévention des feux de forêts, projets d'intensification de l'agriculture axés sur la réduction de la destruction des forêts, et amélioration des emplois hors exploitations agricoles).

Si peu de projets REDD ont été mis en œuvre jusqu'ici - et encore ne s'agissaitil que du secteur volontaire - nombre d'éléments d'information pertinents sont apportés par toute une génération de " projets de conservation et de développement " dont les objectifs sont essentiellement similaires. Diverses raisons expliquent le succès limité de certains de ces projets, entre autres le fait que les auteurs du projet n'aient pas su, par exemple, élaborer des stratégies rattachant clairement les interventions (lancées dans le cadre du projet) aux changements et aux résultats attendus en matière de conservation et de développement (Hughes et Flintan 2001). L'insécurité des titres de propriété foncière d'un grand nombre de résidents pauvres dépendant des forêts constitue l'un des obstacles clé à l'augmentation des revenus des populations rurales à travers des activités forestières durables.

Pour que la REDD soit efficace (sur le plan de la réduction des émissions de $\mathrm{CO}_{2}$ ) et génère des retombées positives substantielles (sur le plan de la réduction de la pauvreté et de l'équité), il faudra qu'elle soit intégrée dans des stratégies de développement économique élargies et s'aligne sur elles. Au nombre de ces stratégies figurent celles qui visent à diminuer la dépendance vis-à-vis des forêts et d'autres ressources naturelles, à savoir la croissance industrielle et de meilleures prestations en matière de services éducatifs et sociaux (Byron et Arnold 1999). Les gouvernements devront coordonner la REDD avec les stratégies nationales de réduction de la pauvreté compte tenu aussi du soutien y afférent apporté par les bailleurs de fonds internationaux.

Enfin, il se peut que les ressources financières liées à la REDD soient utilisées pour appuyer les processus de réforme des gouvernements locaux et l'expansion du capital social, non seulement pour aider à canaliser les flux financiers et à les diriger directement vers les gestionnaires de la foret, mais aussi pour améliorer 
la gouvernance forestière à plus grande échelle. A travers des réformes du gouvernement local, la REDD pourrait améliorer la captation, au niveau local, des recettes provenant de l'exploitation du bois et aider les communautés rurales à gérer la composante locale de ces recettes et à les répartir au mieux des intérêts de la communauté (cf. Larson et Ribot 2006).

\subsection{Bénéfices associés aux droits de I'homme et à la gouvernance}

Des préoccupations liées à des perceptions selon lesquelles la REDD pourrait avoir des retombées négatives sur la protection des droits de l'homme - et ralentir, voire renverser, la tendance naissante à des améliorations en matière de gouvernance forestière au niveau national - sont à l'origine d'une grande partie de l'opposition à l'inclusion de la REDD dans le régime global de protection du climat. En conférant une nouvelle valeur aux terres forestières, la REDD pourrait inciter - tant le gouvernement que les intérêts commerciaux en jeu à opposer un déni actif ou passif aux droits des peuples autochtones et autres communautés dépendantes des forêts à l'accès et au contrôle des ressources de leurs forêts. De nouveaux flux financiers pourraient vraisemblablement attiser les conflits et créer de nouvelles opportunités de corruption.

Par ailleurs, si les paiements effectués au titre de la REDD sont conditionnés par la performance, les données sur l'état et les tendances de la forêt devront être rendues publiques et accessibles ; les gouvernements et les autres acteurs ayant des intérêts commerciaux devront négocier avec les personnes qui auront pouvoir et qualité pour exercer une surveillance efficace sur les ressources de la forêt ; et des mécanismes devront être mis en place pour assurer des transferts financiers transparents et responsables. Plus généralement, cette surveillance plus active (associée aux processus de financement de la REDD) des activités de gestion forestière - à l'échelle internationale - pourrait renforcer l'application des mécanismes de sauvegarde existants. Tout cela pourrait aussi avoir des retombées positives pour les droits de l'homme et la gouvernance.

\subsubsection{Pertinence par rapport à l'architecture de la REDD à l'échelle mondiale}

Une considération qui s'applique à la prise de décision à tous les niveaux est celle du respect des droits liés à la procédure - accès à l'information, participation au processus décisionnel, et accès à la justice - comme spécifié dans le Principe 10 de la Déclaration de Rio (1992). La Convention des Nations Unies sur l'accès à l'information, la participation du public au processus décisionnel et l'accès à la justice en matière d'environnement (dite "Convention d'Aarhus ») établit des principes importants sur la participation des citoyens au processus 
décisionnel relatif à la mise en œuvre de la REDD. Les signataires y sont aussi invités à promouvoir ces principes dans les négociations internationales sur l'environnement.

Dans le contexte des négociations liées à la REDD, le respect des droits de procédure implique l'obligation pour les gouvernements de fournir systématiquement à leurs citoyens des informations pertinentes en temps opportun et des opportunités pour participer de façon substantielle à l'élaboration d'un modèle pour la REDD. Les défenseurs des peuples autochtones ont protesté contre la marginalisation de ces peuples dont la voix n'est pas entendue lors des débats sur la REDD. D'autres groupes ont proposé de mettre en place des groupes consultatifs formels composés de représentants des peuples autochtones et de la société civile afin de conseiller les divers organismes de la CCNUCC sur l'élaboration et la mise en œuvre de la REDD (Rights and Resources Initiative 2008).

Des mécanismes de surveillance et d'évaluation indépendants seront une composante importante de l'architecture de la REDD au niveau mondial pour atténuer le risque " de retombées négatives " en ce qui concerne les droits de l'homme et la gouvernance. La tâche confiée à ces mécanismes pourrait être d'évaluer l'impact des interventions de la REDD sur les droits de l'homme et la gouvernance, et servir ainsi de système d'alerte précoce pour permettre de prompts changements de cap.

Certains éléments du modèle REDD devant être convenus à l'échelle mondiale risquent de multiplier les problèmes liés aux droits de l'homme et à la gouvernance dans le contexte de la mise en œuvre au niveau national ou, à l'inverse, pourraient renforcer les opportunités de retombées positives. Par exemple, s'agissant de la mise en œuvre de la REDD, des approches sous-nationales seraient plus compatibles avec l'application des clauses de sauvegarde et autres instruments de surveillance et de vérification des impacts sur les droits de l'homme. En revanche, les approches nationales offrent de meilleures chances d'utiliser la REDD pour améliorer la gouvernance forestière à travers, par exemple, de vastes réformes foncières. Il pourrait être nécessaire de combiner des éléments des approches centralisées et décentralisées à la gouvernance forestière afin de tirer le meilleur parti possible des avantages et inconvénients de chacune d'entre elles (Colfer et Capistrano 2005).

La REDD peut aussi être liée à divers accords internationaux qui stipulent les obligations des parties en matière de protection des droits de l'homme. Colchester (2008 : 5), par exemple, énumère un certain nombre d'instruments juridiques internationaux relatifs aux droits des peuples autochtones dans lesquels sont affirmés les droits des populations de " posséder, d'utiliser, de mettre en valeur et de contrôler les terres, territoires et ressources qu'ils possèdent et de disposer en toute sécurité de leurs moyens d'existence ». Une description succincte 
des instruments illustrant ces obligations est donnée, à titre d'exemple, dans l'encadré 11.2 ci-après.

\section{Encadré 11.2. Liste illustrative de quelques instruments internationaux sur les droits de l'homme, applicables à la REDD}

La Convention internationale sur les droits économiques, sociaux et culturels affirme « qu'en aucun cas un peuple ne peut être privé de ses propres moyens de subsistance »(Article 1), suggérant impérativement que la REDD n'ait pas pour résultat le déni d'accès à des moyens d'existence basés sur les ressources des forêts.

La Convention internationale sur les droits civils et politiques établit les principes visant à garantir que des violations des droits de l'homme - telles que l'arrestation et la détention arbitraires (Article 9) - n'aient pas pour origine l'application d'approches législatives orientées vers la répression pour réaliser les objectifs de la REDD.

La Déclaration des Nations Unies sur les droits des peuples autochtones proclame que « les Etats mettront en place et appliqueront, en concertation avec les peuples autochtones concernés, un processus équitable, indépendant, ouvert et transparent prenant dûment en compte les lois, traditions, coutumes et régimes fonciers des peuples autochtones, afin de reconnaître les droits des peuples autochtones en ce qui concerne leurs terres, territoires et ressources » (Article 27), processus qui devra nécessairement précéder la mise en œuvre de la REDD.

La Convention sur l'élimination de toutes les formes de discrimination à l'égard des femmes affirme que les plans de développement doivent tenir compte " des problèmes particuliers qui se posent aux femmes rurales et du rôle important que ces femmes jouent dans la survie économique de leurs familles, notamment par leur travail dans les secteurs non monétaires de l'économie » (Article 14) qui revêt une importance substantielle dans le cas de l'utilisation des ressources forestières.

Outre les droits et obligations spécifiés dans les accords internationaux, un ensemble émergent de textes à dispositions non contraignantes et de normes internationales applicables à la REDD se met en place au niveau mondial. Les normes et procédures sont particulièrement importantes pour les droits de l'homme et la gouvernance. Le principe du « consentement libre, informé et préalable» (CLIP) applicable aux communautés touchées par des interventions extérieures de développement est de plus en plus reconnu comme une norme que les gouvernements et les entreprises privées doivent appliquer avant tout projet d'infrastructure ou d'industrie extractive (Colchester et Ferrari 2007). Etablir des normes CLIP dans le contexte de la mise en œuvre de la REDD pourrait garantir des droits de procédure plus importants aux communautés concernées. 


\subsubsection{Opportunités et défis au niveau national}

Tous les changements d'origine REDD au niveau national de la gouvernance forestière sont susceptibles d'entraîner des effets importants sur le bien-être des populations dépendantes des forêts, y compris des populations autochtones. Un grand nombre de communautés pauvres ont progressivement perdu leurs droits depuis les temps coloniaux et ont effectivement été réduites au statut de squatters sur des terres publiques. Chercher à subvenir à ses besoins dans de telles situations implique souvent des comportements qui - même s'ils sont légitimes et nécessaires - sont formellement "illégaux " au regard de la loi, ce qui contribue à la vulnérabilité de ces communautés. Si les pauvres n'ont pas de droits, leur pouvoir de négocier pour obtenir des résultats conformes à leurs intérêts en souffre et ils pâtissent en outre de leur incapacité à défendre les droits qu'ils détiennent (Khan 2006).

Si la proportion des terres forestières désignées pour être utilisées ou détenues par des communautés et des peuples autochtones s'est, depuis peu, accrue dans de modestes proportions, la plupart des forêts des pays susceptibles de participer à un accord mondial sur la REDD restent placées sous le contrôle des gouvernements (Sunderlin et al. 2008). La revalorisation des ressources forestières à travers la mise en place d'un système de droits d'émission pourrait décourager certains gouvernements favorisant les cultures de rentes de concéder aux communautés des droits sur le carbone forestier. S'il était décidé que les paiements effectués au titre de la REDD le seraient sur la base de la performance, la tendance des gouvernements à vouloir retenir ces droits pour leur propre compte serait mise en échec.

Bien que la REDD puisse aussi être l'occasion de progresser dans la voie d'une législation réformée, il faudra accorder une attention particulière aux mécanismes de sauvegarde afin que les intérêts des élites nationales et les intérêts commerciaux à l'échelle mondiale ne l'emportent pas sur les droits des communautés forestières. En conséquence, les investissements internationaux portant sur le renforcement des capacités pour mettre en œuvre la REDD devraient renforcer la capacité des décideurs (notamment les institutions gouvernementales, les entreprises et les organisations non gouvernementales) de protéger les droits de l'homme pendant l'implémentation de la REDD et aussi promouvoir l'habilité des ayants droits de faire valoir leurs droits.

\subsection{Bénéfices associés à la biodiversité et aux autres services de l'écosystème}

La REDD est une source potentielle importante de bénéfices associés à la conservation de la biodiversité et à des retombées positives pour d'autres services de l'écosystème (outre la séquestration du carbone). La conservation 
des forêts tropicales est largement considérée comme ayant systématiquement reçu des dotations insuffisantes au cours des récentes décennies aussi bien du point de vue de l'échelle que de la durée du cycle de financement (Balmford et Whitten 2003), mais les flux financiers associés à la REDD offrent maintenant des opportunités radicalement nouvelles sur ces deux fronts.

S'agissant de la biodiversité, la REDD évite un grand nombre des pièges des projets de boisement et de reboisement $(\mathrm{B} / \mathrm{R})$ qui tendent à favoriser les monocultures d'espèces exotiques. Les monocultures de plantation ont une certaine valeur de biodiversité mais ne représentent en général qu'une petite proportion de la biodiversité des écosystèmes forestiers naturels typiques (Kanowski et al. 2005). Comparée à d'autres projets de boisement et reboisement, la REDD présente aussi probablement l'avantage de ne pas requérir de normes de biodiversité trop contraignantes étant donné que la conservation des forêts est réputée en grande partie propice à la biodiversité.

La REDD devrait aussi favoriser des retombées positives pour les services de conservation hydrologiques et pédologiques. Elle peut aussi contribuer à la lutte contre l'érosion des sols qui a un effet sur la qualité du sol comme de l'eau. A l'échelle mondiale, les trois quarts de l'alimentation utilisable en eau proviennent de bassins versants forestiers (Fischlin et al. 2007). Regrouper la conservation du carbone avec d'autres services d'écosystèmes comme les bassins versants pourrait être à l'origine de scénarios gagnants pour toutes les parties en cause.

Plus généralement, la REDD pourrait rendre possible une conservation à grande échelle des forêts - ce qui aurait aussi des impacts positifs sur le climat allant au-delà des services liés à la séquestration du carbone. Bruijnzeel (2004), par exemple, prédit qu'une conversion à grande échelle des forêts en terres de pâturages en Amazonie pourrait avoir pour résultat une réduction de sept pour cent des précipitations annuelles. Eluder de telles éventualités aurait des retombées positives encore plus grandes pour l'environnement et aiderait à éviter les importantes variations climatiques anticipées susceptibles de se produire (Nepstad 2007).

\subsubsection{Pertinence par rapport à l'architecture de la REDD à l'échelle mondiale}

Dans une certaine mesure et quelle que soit l'échelle, la REDD devrait avoir des effets positifs sur la biodiversité, même si les impacts des divers modèles envisagés sont susceptibles de différer les uns des autres. Le financement de la REDD, en particulier si les fonds proviennent des marchés financiers devrait, selon toutes probabilités, être dirigé vers des zones à fortes émissions de $\mathrm{CO}_{2}$. Cela garantira une efficacité très élevée du carbone mais ces zones 
ne correspondent pas nécessairement aux zones de très grande diversité. Des zones déjà protégées comme les réserves indigènes recouvrant 22 pour cent de l'Amazonie brésilienne et d'autres zones critiques pour la biodiversité comme le Guiana Shield, ne devraient pas bénéficier de ces apports, initialement du moins (da Fonseca et al. 2007). En revanche, les projets REDD de maintien volontaire de stocks pourraient capter une plus grande partie des bénéfices associés à la biodiversité en étendant l'aire géographique ciblée, mais les niveaux de financement resteraient sensiblement plus faibles. Ainsi, quoique les buts visés par le maintien des stocks de carbone et la biodiversité soient largement compatibles, la répartition géographique des fonds pourrait faire l'objet de compromis.

Du point de vue de la biodiversité, les systèmes nationaux sont préférables aux approches basées sur les projets et sont susceptibles de promouvoir une approche plus rationnelle à la planification des paysages. Les économies d'échelle réalisées en matière de systèmes de mesure et de surveillance au niveau national devraient aussi faciliter les choses. Même si elles sont sujettes aux fuites (chapitre 7), les approches au niveau des projets devraient être " propices à la biodiversité " en permettant aux investisseurs de cibler des zones spécifiques ayant une grande valeur sur le plan de la biodiversité. Dans le Mercado Park Noel Kempff en Bolivie, par exemple, qui est l'un des rares exemples d'un projet volontaire de la REDD, des fragments de forêts ont été soignés pour devenir des unités plus écologiquement cohérentes malgré les préoccupations concernant l'apparition de fuites possibles au-delà des limites du parc (Robertson et Wunder 2005).

La mesure dans laquelle les flux financiers de la REDD s'écouleront vers les forêts sèches dépendra d'un certain nombre de facteurs au niveau mondial, notamment des niveaux de référence, des mécanismes de financement et de l'inclusion ou non - dans le champ d'application - de la dégradation évitée. Si le modèle REDD privilégie le ciblage de zones boisées visées par la Convention des Nations Unies sur la lutte contre la désertification (CNULD/CCD), cette décision pourrait avoir des retombées positives particulièrement importantes pour combattre l'érosion des sols dans ces zones. Un tel ciblage pourrait toutefois impliquer des compromis sur le plan de l'efficacité et de l'efficience globale du mécanisme de la REDD, étant donné le stock de carbone souterrain sensiblement plus faible dans ces zones que dans les forêts tropicales humides.

La nécessité de maintenir les fonctions majeures de l'écosystème indique qu'il faut veiller à une planification coordonnée des paysages au niveau international - ce qui dépasse vraisemblablement le cadre d'un accord ciblant l'atténuation des émissions de $\mathrm{CO}_{2}$. Toutefois, un certain nombre d'accords internationaux applicables aux prestations et activités envisagées pour la REDD encouragent l'harmonisation avec des objectifs environnementaux plus larges aux échelles nationale et régionale. Au nombre de ces accords figurent la Convention des 
Nations Unies sur la diversité biologique, la CCNUCC et la Convention de Ramsar sur les zones humides.

\subsubsection{Opportunités et défis au niveau national}

La mesure dans laquelle les politiques et mesures de la REDD mises en œuvre au niveau national auront des répercussions sur la biodiversité et les autres services de l'écosystème dépendront des options et stratégies existantes en matière d'utilisation des terres, des types d'activités encouragées ou prohibées ainsi que de leur localisation géographique. Le tout sera très dépendant des facteurs déterminants sous-jacents du déboisement et de l'impact sur l'environnement des utilisations alternatives des terres forestières.

Dans les zones boisées qui, par exemple, auraient été soumises à des pratiques d'exploitation classiques, les fonds de la REDD pourraient contribuer à la conservation de la biodiversité s'ils sont utilisés de façon à encourager un impact réduit de l'abattage sur l'environnement (Meijaard et al. 2005). Les retombées positives seraient encore plus importantes si les forêts déjà exploitées risquaient autrement d'être converties en terres de production agricole en l'absence d'un financement REDD.

Les stratégies de la REDD visant à dissuader les agriculteurs de continuer leurs pratiques culturales cycliques destructrices peuvent apparaître comme positives pour la biodiversité mais il reste encore à en évaluer l'impact pour chaque situation. La biodiversité des jachères arbustives agricoles, par exemple, typiques de la culture itinérante, peut être beaucoup plus grande que celle de produits comparables de l'agriculture permanente (Tutin et Fernandez 1985). Il se peut qu'il soit préférable, du point de vue de la biodiversité, d'améliorer la productivité des pratiques cycliques et/ou des systèmes d'agroforesterie. Les cultures pérennes comme le café ou le cacao peuvent permettre cette connectivité qui aboutit au maintien des effets de l'écosystème, en particulier dans le cas où des essences tolérantes ou dépendantes de l'ombre sont employées, mais elles pourraient nécessiter des traitements chimiques lourds pour supprimer les attaques fongiques et celles des ravageurs. Pour optimiser les objectifs de séquestration du carbone de la REDD et ceux des retombées positives pour l'environnement, il sera donc nécessaire de prendre en compte les mesures et les résultats pour la totalité du cycle agricole. 


\subsection{Conclusion}

Le défi auquel est confrontée la communauté internationale est celui de veiller à ce que l'architecture qui sera mise en place à l'échelle mondiale par la CCNUCC ouvre des opportunités aux pays en développement - et ne les en prive pas - pour mettre en œuvre la REDD de façon à obtenir des retombées positives pour la réduction de la pauvreté, la protection des droits de l'homme et les services de l'écosystème sans émissions de $\mathrm{CO}_{2}$, et à éviter des effets préjudiciables. Plus grande sera la probabilité de retombées positives importantes, moindres seront les risques, notamment si l'afflux de capitaux financiers et la mise en œuvre de la REDD au niveau national - ainsi que les stratégies de développement national - sont harmonisés avec d'autres engagements internationaux préexistants et des normes émergentes.

L'un des plus grands défis à relever sera de définir des normes et procédures appropriées - notamment en matière de mécanismes d'évaluation, de surveillance et de vérification - pour veiller à ce que soient dûment pris en compte les risques et les opportunités sans imposer de coûts de transaction excessifs qui iraient à l'encontre de la réalisation des objectifs de la REDD et de ses retombées positives. 



\section{Appendice}

\section{Récapitulatif des propositions REDD soumises à la CCNUCC ${ }^{1}$}

Philippe Guizol et Stibniati Atmadja

\begin{tabular}{|c|c|c|}
\hline Questions & Opinions & Appuyées par \\
\hline \multicolumn{3}{|c|}{ Enjeux clé pour l'architecture du modèle } \\
\hline \multirow[t]{4}{*}{$\begin{array}{l}\text { Principes } \\
\text { directeurs }\end{array}$} & $\begin{array}{l}\text { Responsabilités communes mais } \\
\text { différenciées : des circonstances } \\
\text { nationales différentes d'un pays } \\
\text { à l'autre détermineront différents } \\
\text { niveaux de participation et de } \\
\text { nouvelles incitations ciblées } \\
\text { et positives. }\end{array}$ & $\begin{array}{l}\text { Costa Rica (avril 08); } \\
\text { PNG - GRULAC - } \\
\text { Costa Rica (mars 07); } \\
\text { Mexique (avril 08); UE } \\
\text { (juillet 08) }\end{array}$ \\
\hline & $\begin{array}{l}\text { L'accent est mis sur la souveraineté } \\
\text { ou le droit d'une nation à l'auto- } \\
\text { détermination et au développement } \\
\text { économique. La participation à } \\
\text { tout futur mécanisme devrait être } \\
\text { volontaire et prendre en compte } \\
\text { les circonstances nationales et les } \\
\text { politiques et initiatives existantes. }\end{array}$ & $\begin{array}{l}\text { Costa Rica (avril 08); } \\
\text { PNG (avril 08) ; Groupe } \\
\text { de travail ACCRA (août } \\
\text { 08) ; Mexique (avril 08) }\end{array}$ \\
\hline & $\begin{array}{l}\text { Equité à l'intérieur des pays : il } \\
\text { conviendra de s'assurer de la } \\
\text { participation des parties prenantes, } \\
\text { des communautés locales et } \\
\text { des populations autochtones. } \\
\text { La répartition des bénéfices de } \\
\text { la REDD aux acteurs locaux doit } \\
\text { être transparente. }\end{array}$ & $\begin{array}{l}\text { Groupe de travail } \\
\text { ACCRA (août 08); } \\
\text { Japon (août 08) ; PNG } \\
\text { (avril 08) }\end{array}$ \\
\hline & $\begin{array}{l}\text { Equité d'un pays à l'autre. Toute } \\
\text { action anticipée pour réduire la DD } \\
\text { doit être récompensée. }\end{array}$ & $\begin{array}{l}\text { Costa Rica (avril 08); } \\
\text { CfRN (septembre 07); } \\
\text { Mexique (avril 08) }\end{array}$ \\
\hline
\end{tabular}

Les soumissions peuvent être consultées sur le site : http ://unfccc.int. Cet appendice a été mis à jour le 19 novembre 2008 . 


\begin{tabular}{|c|c|c|}
\hline Questions & Opinions & Appuyées par \\
\hline \multicolumn{3}{|c|}{ Enjeux clé pour l'architecture du modèle (suite) } \\
\hline \multirow[t]{5}{*}{$\begin{array}{l}\text { Principes } \\
\text { directeurs (suite) }\end{array}$} & $\begin{array}{l}\text { Les actions complémentaires de } \\
\text { la REDD (le Fonds Vert) doivent } \\
\text { englober tous les pays. }\end{array}$ & Mexique (juin 08) \\
\hline & $\begin{array}{l}\text { La REDD doit aussi inclure les pays à } \\
\text { faible déforestation. }\end{array}$ & Greenpeace (mars 08) \\
\hline & $\begin{array}{l}\text { Les connaissances actuelles } \\
\text { en matière de questions } \\
\text { méthodologiques sont suffisantes } \\
\text { pour entamer des discussions sur les } \\
\text { approches politiques et stratégiques } \\
\text { et les incitations positives. }\end{array}$ & $\begin{array}{l}\text { Groupe de travail } \\
\text { ACCRA (août 08) }\end{array}$ \\
\hline & $\begin{array}{l}\text { Besoin de soutien immédiat aux } \\
\text { pays-hôtes pour renforcer la } \\
\text { gouvernance et les capacités à } \\
\text { tous les niveaux dans les pays en } \\
\text { développement. Inclut le besoin } \\
\text { de promouvoir le transfert de } \\
\text { technologie et la coopération } \\
\text { technique Nord-Sud et Sud-Sud. }\end{array}$ & $\begin{array}{l}\text { Groupe de travail } \\
\text { ACCRA (août 08); } \\
\text { Brésil (mars 07); } \\
\text { COMIFAC (avril 08); } \\
\text { Costa Rica (avril 08); } \\
\text { CfRN (septembre 07); } \\
\text { Mexique (août 08); } \\
\text { PNG (avril 08) }\end{array}$ \\
\hline & $\begin{array}{l}\text { La période s'étendant jusqu'à } \\
2012 \text { devrait être consacrée à des } \\
\text { « activités de démonstration » et } \\
\text { à l'élaboration d'un « inventaire } \\
\text { national du carbone forestier » pour } \\
\text { faciliter l'intégration des approches } \\
\text { et des modèles politico-stratégiques } \\
\text { des incitations qui seront proposées } \\
\text { pour la REDD à la COP 15. Dans } \\
\text { la période postérieure à 2012, il } \\
\text { faudrait recentrer l'intérêt sur les } \\
\text { politiques et incitations positives } \\
\text { pour la REDD. }\end{array}$ & UE (juillet 08) \\
\hline \multirow[t]{3}{*}{$\begin{array}{l}\text { Cadre } \\
\text { institutionnel } \\
\text { mondial }\end{array}$} & $\begin{array}{l}\text { Traité séparé pour la REDD, distinct } \\
\text { du Protocole de Kyoto. }\end{array}$ & $\begin{array}{l}\text { Brésil (mars 07) ; CCAP } \\
\text { (août 07) ; Nouvelle } \\
\text {-Zélande séparé mais } \\
\text { lié au Protocole de } \\
\text { Kyoto (avril 08) }\end{array}$ \\
\hline & $\begin{array}{l}\text { La REDD doit relever de la CCNUCC } \\
\text { et être intégrée dans un accord } \\
\text { post- } 2012 \text {. }\end{array}$ & $\begin{array}{l}\text { Mexique (août 08); } \\
\text { CfRN (septembre } \\
\text { 07); Greenpeace } \\
\text { (mars 08) Norvège } \\
\text { (septembre 08) }\end{array}$ \\
\hline & $\begin{array}{l}\text { La REDD doit relever d'une } \\
\text { institution préexistante (non- } \\
\text { CCNUCC), par ex. FCPF, UN-REDD. }\end{array}$ & $\begin{array}{l}\text { FAO, PNUD, PNUE } \\
\text { (avril 08); PNG } \\
\text { (avril 08) }\end{array}$ \\
\hline
\end{tabular}




\begin{tabular}{|c|c|c|}
\hline Questions & Opinions & Appuyées par \\
\hline \multicolumn{3}{|c|}{ Enjeux clé pour l'architecture du modèle (suite) } \\
\hline \multirow[t]{4}{*}{$\begin{array}{l}\text { Champ } \\
\text { d'application de la } \\
\text { REDD }\end{array}$} & $\begin{array}{l}\text { Activités de boisement/reboisement } \\
\text { (B/R) devraient être incluses dans } \\
\text { tout projet REDD. }\end{array}$ & $\begin{array}{l}\text { Inde (avril 08) ; Japon } \\
\text { (août 08) }\end{array}$ \\
\hline & $\begin{array}{l}\text { Tout projet REDD devrait inclure le } \\
\text { renforcement des stocks de carbone } \\
\text { et la gestion durable des forêts. }\end{array}$ & $\begin{array}{l}\text { Argentine, Honduras, } \\
\text { Panama, Paraguay } \\
\text { et Pérou (avril 08); } \\
\text { Costa Rica (avril 08) ; } \\
\text { Inde (avril 08) ; Japon } \\
\text { (août 08) ; Népal } \\
\text { (août 08) ; Indonésie } \\
\text { (avril 08) ; Norvège } \\
\text { (septembre 08) }\end{array}$ \\
\hline & $\begin{array}{l}\text { La gestion durable des forêts, le } \\
\text { renforcement des stocks de carbone } \\
\text { et la conservation des forêts ne } \\
\text { sont pas des activités qui, par } \\
\text { nature, sont directement à même } \\
\text { d'éviter les émissions dues à la } \\
\text { déforestation. }\end{array}$ & Colombie (avril 08) \\
\hline & $\begin{array}{l}\text { Les actions additionnelles doivent } \\
\text { compléter celles de la REDD : en } \\
\text { tant que mesures de gestion des } \\
\text { forêts, de conservation du carbone, } \\
\text { de lutte contre les feux de forêts } \\
\text { et de séquestration du carbone } \\
\text { qui ont pour résultat des bénéfices } \\
\text { associés sur le plan local et à } \\
\text { l'échelle mondiale. }\end{array}$ & $\begin{array}{l}\text { Mexique (août 08); } \\
\text { Japon (avril 08); UE } \\
\text { (juillet 08) ; Indonésie } \\
\text { (avril 08) ; Inde (avril } \\
\text { 08) ; Népal (avril 08); } \\
\text { COMIFAC (avril 08) }\end{array}$ \\
\hline \multirow[t]{2}{*}{$\begin{array}{l}\text { Activités } \\
\text { comptabilisées au } \\
\text { titre de la REDD }\end{array}$} & $\begin{array}{l}\text { Basées sur les intrants : les } \\
\text { paiements sont effectués sous } \\
\text { réserve d'intrants mis en œuvre } \\
\text { pour produire un résultat souhaité } \\
\text { même si le résultat ne peut être } \\
\text { mesuré directement, par ex. gestion } \\
\text { forestière participative, prévention } \\
\text { des feux de forêts et lutte contre } \\
\text { l'abattage illégal. }\end{array}$ & Japon (août 08) \\
\hline & $\begin{array}{l}\text { Basées sur les résultats : attribution } \\
\text { de crédits au titre du renforcement } \\
\text { des stocks de carbone et du } \\
\text { maintien des stocks de référence. }\end{array}$ & Inde (avril 08) \\
\hline
\end{tabular}




\begin{tabular}{|c|c|c|}
\hline Questions & Opinions & Appuyées par \\
\hline \multicolumn{3}{|c|}{ Enjeux clé pour l'architecture du modèle (suite) } \\
\hline $\begin{array}{l}\text { Activités } \\
\text { comptabilisées au } \\
\text { titre de la REDD } \\
\text { (suite) }\end{array}$ & $\begin{array}{l}\text { Approches de comptabilisation } \\
\text { mixtes : (i) activités de déforestation } \\
\text { et de dégradation des forêts (DD) } \\
\text { non planifiées : crédits attribués } \\
\text { sur la base du niveau de réduction } \\
\text { d'émissions des activités par } \\
\text { rapport au niveau historique } \\
\text { d'émissions de ces mêmes activités } \\
\text { (comme la gestion des forêts) ; et } \\
\text { (ii) activités DD planifiées : le stock } \\
\text { de carbone sauvé en raison de la } \\
\text { non application d'une conversion } \\
\text { planifiée des forêts. }\end{array}$ & Indonésie (avril 08) \\
\hline \multicolumn{3}{|c|}{ Coûts, potentiel et risques } \\
\hline \multirow[t]{3}{*}{ Coûts } & $\begin{array}{l}\text { Importance de l'adoption de } \\
\text { moyens justes et rentables pour } \\
\text { réduire les émissions dues à la DD } \\
\text { dans les pays en développement. }\end{array}$ & $\begin{array}{l}\text { Groupe de travail } \\
\text { ACCRA (août 08) ; } \\
\text { COMIFAC (avril 08) }\end{array}$ \\
\hline & $\begin{array}{l}\text { Coûts de transaction liés aux } \\
\text { capacités de réponse de la } \\
\text { REDD (par ex. renforcement } \\
\text { des capacités). }\end{array}$ & $\begin{array}{l}\text { Bassin du Congo (mars } \\
07) ; C f R N \text { (septembre } \\
07) \text {; Indonésie } \\
\text { (août 08) }\end{array}$ \\
\hline & $\begin{array}{l}\text { Coûts associés à la mise en } \\
\text { conformité avec les normes } \\
\text { internationales (par ex. surveillance). }\end{array}$ & $\begin{array}{l}\text { PNG (avril 08); } \\
\text { BioCarbon (Banque } \\
\text { Mondiale août 08) }\end{array}$ \\
\hline \multirow[t]{3}{*}{ Risques } & $\begin{array}{l}\text { Le marché REDD pourrait mettre } \\
\text { en péril l'intégrité du marché du } \\
\text { carbone existant et les efforts } \\
\text { pour réduire l'utilisation des } \\
\text { combustibles fossiles. }\end{array}$ & $\begin{array}{l}\text { CCAP (août 07); } \\
\text { Nouvell-Zélande } \\
\text { (avril 08) }\end{array}$ \\
\hline & $\begin{array}{l}\text { L'offre et la demande de crédits } \\
\text { REDD n'est pas connue, ce qui } \\
\text { entraîne une réticence au risque } \\
\text { de la part des acheteurs et } \\
\text { des vendeurs. }\end{array}$ & $\begin{array}{l}\text { CCAP (août 07) ; CISDL } \\
\text { et GPPI (février 07); } \\
\text { FAO, PNUD, PNUE } \\
\text { (avril 08) ; Nouvelle- } \\
\text { Zélande (avril 08) }\end{array}$ \\
\hline & $\begin{array}{l}\text { Les questions foncières devraient } \\
\text { être abordées sous l'angle des } \\
\text { réformes institutionnelles et de } \\
\text { l'engagement des communautés } \\
\text { en faisant valoir qu'agir à travers } \\
\text { ces deux piliers d'organisation de la } \\
\text { société est une façon d'atténuer les } \\
\text { risques de conflit. }\end{array}$ & $\begin{array}{l}\text { FAO, PNUD, PNUE } \\
\text { (avril 08) }\end{array}$ \\
\hline
\end{tabular}




\begin{tabular}{|c|c|c|}
\hline Questions & Opinions & Appuyées par \\
\hline \multicolumn{3}{|c|}{ Coûts, potentiel et risques (suite) } \\
\hline \multirow[t]{2}{*}{$\begin{array}{l}\text { Risques } \\
\text { (suite) }\end{array}$} & $\begin{array}{l}\text { La stratégie de la REDD au niveau } \\
\text { national devrait être intégrée dans } \\
\text { la planification nationale existante } \\
\text { pour le développement en tant } \\
\text { qu'outil permettant d'atténuer les } \\
\text { risques liés aux prestations. }\end{array}$ & $\begin{array}{l}\text { FAO, PNUD, PNUE } \\
\text { (avril 08) }\end{array}$ \\
\hline & $\begin{array}{l}\text { Les risques liés aux « réductions } \\
\text { fictives » peuvent être atténués en } \\
\text { stockant les crédits émis pendant } \\
\text { une période afin de les utiliser } \\
\text { pendant une autre. }\end{array}$ & $\begin{array}{l}\text { CISDL et GPPI (février } \\
07 \text { ) ; Costa Rica } \\
\text { (avril 08) }\end{array}$ \\
\hline \multicolumn{3}{|c|}{ Echelle } \\
\hline \multirow[t]{2}{*}{$\begin{array}{l}\text { Approches au } \\
\text { niveau sous- } \\
\text { national/des } \\
\text { projets }\end{array}$} & $\begin{array}{l}\text { Les approches au titre des projets } \\
\text { devraient pouvoir commencer } \\
\text { précocement et bénéficier d'une } \\
\text { large participation là où elles sont } \\
\text { attrayantes pour les investisseurs } \\
\text { privés, mais elles pâtissent de fuites } \\
\text { au niveau national et couvrent des } \\
\text { zones forestières limitées. }\end{array}$ & $\begin{array}{l}\text { BioCarbon (Banque } \\
\text { Mondiale) (août 08) }\end{array}$ \\
\hline & $\begin{array}{l}\text { Les approches sous-nationales } \\
\text { aident à engranger l'expérience } \\
\text { nécessaire pour mettre en œuvre les } \\
\text { approches nationales. }\end{array}$ & COMIFAC (avril 08) \\
\hline $\begin{array}{l}\text { Approches } \\
\text { nationales }\end{array}$ & $\begin{array}{l}\text { Les approches nationales } \\
\text { permettent de poursuivre une } \\
\text { vaste panoplie de politiques, de } \\
\text { capturer les fuites et de créer un } \\
\text { sentiment d'appropriation au } \\
\text { niveau national, mais elles ne } \\
\text { seront réalisables que dans un } \\
\text { petit nombre de pays à court et } \\
\text { moyen terme. Les approches sous- } \\
\text { nationales pourraient être adaptées } \\
\text { à certaines circonstances nationales } \\
\text { en tant qu'étape vers la promotion } \\
\text { d'approches nationales, de niveaux } \\
\text { de référence et d'estimations. }\end{array}$ & $\begin{array}{l}\text { Brésil (mars 07); } \\
\text { CCAP (août 07); CfRN } \\
\text { (septembre 07); CISDL } \\
\text { et GPPI (février 07); } \\
\text { Colombie (avril 08); } \\
\text { COMIFAC (avril 08); } \\
\text { UE (avril 08) ; FAO, } \\
\text { PNUD, PNUE (avril } \\
\text { 08) ; Greenpeace (mars } \\
\text { 08); Inde (avril 08); } \\
\text { Indonésie (avril 08); } \\
\text { Nouvelle-Zélande } \\
\text { (avril 08) ; Norvège } \\
\text { (septembre 08) }\end{array}$ \\
\hline
\end{tabular}




\begin{tabular}{|c|c|c|}
\hline Questions & Opinions & Appuyées par \\
\hline \multicolumn{3}{|c|}{ Echelle (suite) } \\
\hline $\begin{array}{l}\text { Approche } \\
\text { combinée }\end{array}$ & $\begin{array}{l}\text { Une approche combinée est un } \\
\text { mécanisme d'utilisation flexible et } \\
\text { de soutien des projets qui permet } \\
\text { des actions anticipées; peuvent } \\
\text { aussi être engagées à ce titre des } \\
\text { actions relevant soit d'une approche } \\
\text { nationale soit d'une combinaison } \\
\text { d'approches à la fois nationales } \\
\text { et basées sur des projets dans } \\
\text { un système où les crédits REDD } \\
\text { sont partagés entre projets et } \\
\text { gouvernements. }\end{array}$ & $\begin{array}{l}\text { Costa Rica (avril 08); } \\
\text { Colombie (avril 08); } \\
\text { Paraguay, Argentine, } \\
\text { Honduras, Panama, } \\
\text { Pérou (avril 08) ; PNG } \\
\text { (avril 08) ; Népal } \\
\text { (avril 08) }\end{array}$ \\
\hline $\begin{array}{l}\text { De l'approche } \\
\text { globale à } \\
\text { l'approche locale }\end{array}$ & $\begin{array}{l}\text { Partage des tâches entre acteurs } \\
\text { aux niveaux global, national, sous- } \\
\text { national et local. }\end{array}$ & Suisse (avril 08) \\
\hline \multicolumn{3}{|c|}{ Financement } \\
\hline \multirow[t]{3}{*}{$\begin{array}{l}\text { Sources de } \\
\text { financement. } \\
\text { Autres que le } \\
\text { marché, double } \\
\text { (à la fois marché } \\
\text { et autres que le } \\
\text { marché), basées } \\
\text { sur le marché }\end{array}$} & $\begin{array}{l}\text { Préférence pour un financement } \\
\text { non basé sur le marché pour } \\
\text { les incitations aux réductions } \\
\text { d'émissions, sources de financement } \\
\text { nouvelles et additionnelles (de pays } \\
\text { visés par l'Annexe I), fonds publics } \\
\text { (APD), donations, mécanismes } \\
\text { financiers au titre de la CCNUCC. }\end{array}$ & Brésil (mars 07) \\
\hline & $\begin{array}{l}\text { Double, avec un mécanisme de } \\
\text { financement principal à travers le } \\
\text { Fonds d'affectation spécial multi- } \\
\text { donateurs (FASM/MDTF). }\end{array}$ & $\begin{array}{l}\text { FAO, PNUD, PNUE } \\
\text { (avril 08) }\end{array}$ \\
\hline & $\begin{array}{l}\text { Double. Fonds supplémentaires } \\
\text { consacrés aux actions anticipées ou } \\
\text { sources de financement multiples. } \\
\text { Les sources de financement } \\
\text { pourraient être plus différenciées } \\
\text { avec le temps. Fonds (autres que } \\
\text { ceux du marché) à court et moyen } \\
\text { terme pour financer le renforcement } \\
\text { des capacités et autres coûts de } \\
\text { transaction, et solutions de marché } \\
\text { à long terme pour financer les } \\
\text { activités effectives de réduction } \\
\text { d'émissions de } \mathrm{CO}_{2} \text {. }\end{array}$ & $\begin{array}{l}\text { COMIFAC (avril 08); } \\
\text { CCAP (août 07) ; UE } \\
\text { (avril 08, juillet 08); } \\
\text { CfRN (septembre 07); } \\
\text { Nouvelle-Zélande } \\
\text { (avril 08) ; PNG (mars } \\
\text { 07) ; PNG (avril 08); } \\
\text { Greenpeace (décembre } \\
\text { 07) ; Norvège } \\
\text { (septembre 08) }\end{array}$ \\
\hline
\end{tabular}




\begin{tabular}{|c|c|c|}
\hline Questions & Opinions & Appuyées par \\
\hline \multicolumn{3}{|c|}{ Financement (suite) } \\
\hline \multirow[t]{4}{*}{$\begin{array}{l}\text { Sources de } \\
\text { financement. } \\
\text { Autres que le } \\
\text { marché, double } \\
\text { (à la fois marché } \\
\text { et autres que le } \\
\text { marché), basées } \\
\text { sur le marché } \\
\text { (suite) }\end{array}$} & $\begin{array}{l}\text { Double. Le financement par des } \\
\text { sources autres que le marché } \\
\text { peut aussi être utilisé à l'appui de } \\
\text { nouveaux Fonds d'investissements } \\
\text { comme les Community Forest } \\
\text { Retention Trust Fund (CFRTD), } \\
\text { International Forest Retention Fund } \\
\text { (IFRD), Compensated Conservation et } \\
\text { World Climate Change Fund (WCCF). }\end{array}$ & $\begin{array}{l}\text { PNG (mars 07) ; } \\
\text { Mexique juin 08) }\end{array}$ \\
\hline & $\begin{array}{l}\text { Préférence pour un financement } \\
\text { basé sur le marché plutôt que sur } \\
\text { des fonds d'investissement, mais } \\
\text { ouvert aux deux options. }\end{array}$ & $\begin{array}{l}\text { Nouvelle-Zélande } \\
\text { (avril 08) }\end{array}$ \\
\hline & $\begin{array}{l}\text { Préférence pour un financement } \\
\text { basé sur le marché provenant } \\
\text { de la vente des crédits de } \\
\text { réduction d'émissions. }\end{array}$ & $\begin{array}{l}\text { Australie (avril 08); } \\
\text { Colombie (avril 08); } \\
\text { Costa Rica (avril 08); } \\
\text { CfRN (septembre 07); } \\
\text { PNG (mars 07) ; CISDL } \\
\text { et GPPI (février 07) }\end{array}$ \\
\hline & $\begin{array}{l}\text { Inclut un financement provenant de } \\
\text { la vente aux enchères des permis } \\
\text { d'émissions de } \mathrm{CO}_{2} \text {. }\end{array}$ & $\begin{array}{l}\text { Norvège } \\
\text { (septembre 08) }\end{array}$ \\
\hline \multirow[t]{2}{*}{$\begin{array}{l}\text { Bénéficiaires du } \\
\text { financement }\end{array}$} & $\begin{array}{l}\text { Ne devrait pas être limité aux pays } \\
\text { où les réductions des émissions } \\
\text { liées à la déforestation sont les } \\
\text { moins onéreuses, ni aux pays dotés } \\
\text { des plus grandes capacités de } \\
\text { surveillance et, par conséquent, les } \\
\text { moins enclins à pâtir de risques liés } \\
\text { à la non-permanence. }\end{array}$ & Greenpeace (mars 08) \\
\hline & $\begin{array}{l}\text { Fonds spéciaux axés sur le } \\
\text { renforcement des capacités dans les } \\
\text { pays en développement }\end{array}$ & Mexique (août 08) \\
\hline \multirow{2}{*}{$\begin{array}{l}\text { Source de } \\
\text { financement : pays } \\
\text { visés à I'Annexe I } \\
\text { vs. pas seulement } \\
\text { les pays visés à } \\
\text { l'Annexe I }\end{array}$} & $\begin{array}{l}\text { Source de ressources financières } \\
\text { pour la REDD en provenance des } \\
\text { pays visés à l'Annexe I. }\end{array}$ & Brésil (mars 07) \\
\hline & $\begin{array}{l}\text { La source de financement destinée } \\
\text { à alimenter la REDD n'est pas } \\
\text { présumée être exclusivement } \\
\text { limitée aux pays visés à I'Annexe I. }\end{array}$ & $\begin{array}{l}\text { Nouvelle-Zélande } \\
\text { (avril 08); Mexique } \\
\text { (juin 08) }\end{array}$ \\
\hline
\end{tabular}




\begin{tabular}{|c|c|c|}
\hline Questions & Opinions & Appuyées par \\
\hline \multicolumn{3}{|c|}{ Financement (suite) } \\
\hline \multirow[t]{4}{*}{$\begin{array}{l}\text { Fongibilité } \\
\text { des crédits } \\
\text { de réduction } \\
\text { d'émissions }\end{array}$} & $\begin{array}{l}\text { Fongibilité. La REDD produit des } \\
\text { crédits commercialisables pour les } \\
\text { réductions des pays visés à l'Annexe } \\
\text { I. Les partisans de la fongibilité } \\
\text { veulent que la REDD s'appuie sur } \\
\text { un marché à large base et craignent } \\
\text { que le financement de la REDD soit } \\
\text { insuffisant, comme dans le cas du } \\
\text { MDP, si les crédits REDD ne sont pas } \\
\text { échangeables sur les principaux } \\
\text { marchés du carbone. }\end{array}$ & $\begin{array}{l}\text { Chili (août 07), } \\
\text { Paraguay, Argentine, } \\
\text { Honduras, Panama, } \\
\text { Pérou (avril 08); } \\
\text { Colombie (avril 08); } \\
\text { CfRN (septembre } \\
07 \text {, avril 08) ; CISDL } \\
\text { et GPPI (février 07); } \\
\text { Mexique (août 08) }\end{array}$ \\
\hline & $\begin{array}{l}\text { Non fongible. Les partisans de } \\
\text { la non-fongibilité craignent que } \\
\text { de gros volumes de crédits bon } \\
\text { marché déstabilisent le marché } \\
\text { réglementaire du carbone et } \\
\text { réduisent les prix, et/ou que les } \\
\text { pays industriels prennent moins } \\
\text { de mesures pour réduire leurs } \\
\text { taux d'émissions en provenance } \\
\text { des combustibles fossiles et de } \\
\text { leurs industries. }\end{array}$ & $\begin{array}{l}\text { Brésil (mars } \\
07) ; \text { Greenpeace } \\
\text { (décembre 07) }\end{array}$ \\
\hline & $\begin{array}{l}\text { Fongibilité limitée. Par exemple, } \\
\text { pour prévenir tout risque } \\
\text { d'inondation des marchés, } \\
\text { des limites spécifiques ou } \\
\text { plafonnements devraient être } \\
\text { imposés sur les montants des } \\
\text { crédits éligibles. }\end{array}$ & $\begin{array}{l}\text { BioCarbon (Banque } \\
\text { Mondiale août 08) }\end{array}$ \\
\hline & $\begin{array}{l}\text { Non fongible à court et moyen } \\
\text { terme (avant } 2012 \text { ou 2020). La } \\
\text { fongibilité future est conditionnée } \\
\text { par l'engagement des pays visés à } \\
\text { l'Annexe } 1 \text { à se fixer des objectifs de } \\
\text { réduction plus élevés. }\end{array}$ & $\begin{array}{l}\text { CCAP (août 07) non } \\
\text { fongible jusqu'en } \\
2012 \text {; UE (avril 08); } \\
\text { UE (juillet 08) ; UE } \\
\text { (octobre 08) non } \\
\text { fongible jusqu'en } 2020\end{array}$ \\
\hline \multicolumn{3}{|c|}{ Niveaux de référence } \\
\hline $\begin{array}{l}\text { Base de } \\
\text { financement }\end{array}$ & $\begin{array}{l}\text { Financement basé sur les } \\
\text { besoins financiers spécifiques } \\
\text { des pays REDD et/ou sur les } \\
\text { circonstances nationales. }\end{array}$ & $\begin{array}{l}\text { PNG (avril 08) ; UE } \\
\text { (juillet 08); Paraguay, } \\
\text { Argentine, Honduras, } \\
\text { Panama, Pérou } \\
\text { (avril 08) }\end{array}$ \\
\hline
\end{tabular}




\begin{tabular}{|c|c|c|}
\hline Questions & Opinions & Appuyées par \\
\hline \multicolumn{3}{|c|}{ Niveaux de référence (suite) } \\
\hline \multirow[t]{7}{*}{$\begin{array}{l}\text { Méthode de } \\
\text { fixation du niveau } \\
\text { de référence }\end{array}$} & $\begin{array}{l}\text { Recommande d'utiliser des données } \\
\text { de déforestation historiques. }\end{array}$ & $\begin{array}{l}\text { Plan d'Action de Bali } \\
\text { (décembre 07) ; Costa } \\
\text { Rica (avril 08) ; FAO, } \\
\text { PNUD, PNUE (avril 08); } \\
\text { CCAP (août 07) ; Brésil } \\
\text { (mars 07) ; Indonésie } \\
\text { (avril 08) ; Inde (avril } \\
\text { 08) ; Nouvelle-Zélande } \\
\text { (avril 08) ; PNG (avril } \\
\text { 08) ; UE (avril 08); } \\
\text { Greenpeace (mars } \\
\text { 08) ; CfRN (septembre } \\
\text { 07); Paraguay, } \\
\text { Argentine, Honduras, } \\
\text { Panama, Pérou (avril } \\
\text { 08) (poursuite de } \\
\text { I'approche combinée : } \\
\text { soumission par le } \\
\text { Paraguay et al. 07) ; UE } \\
\text { (juillet 08) ; Norvège } \\
\text { (septembre 08) }\end{array}$ \\
\hline & $\begin{array}{l}\text { Niveaux de référence distincts pour } \\
\text { la déforestation (hectares par forêt } \\
\text { perdue/an), et la dégradation des } \\
\text { forêts (tonnes de carbone/ha/an). }\end{array}$ & Népal (avril 08) \\
\hline & $\begin{array}{l}\text { Aire forestière de conservation } \\
\text { (protégée) pour servir de niveau de } \\
\text { référence dans l'approche basée sur } \\
\text { les stocks. }\end{array}$ & $\begin{array}{l}\text { CISDL et GPPI } \\
\text { (février 07) }\end{array}$ \\
\hline & $\begin{array}{l}\text { Recommande d'utiliser } \\
\text { dorénavant des tendances } \\
\text { projetées/d'extrapoler les } \\
\text { tendances historiques. }\end{array}$ & $\begin{array}{l}\text { Indonésie (avril } \\
08) ; \text { CISDL et GPPI } \\
\text { (février 07) ; COMIFAC } \\
\text { (avril 08); Colombie } \\
\text { (avril 08) }\end{array}$ \\
\hline & $\begin{array}{l}\text { Les mécanismes nationaux } \\
\text { permettront de mettre au point des } \\
\text { niveaux de référence plus exacts. }\end{array}$ & $\begin{array}{l}\text { Nouvelle Zélande } \\
\text { (avril 08) }\end{array}$ \\
\hline & $\begin{array}{l}\text { Elaborer un système de niveaux de } \\
\text { référence combinés pour différentes } \\
\text { zones d'un pays, où le volume total } \\
\text { des émissions et les quantités de } \\
\text { carbone accumulées dans les puits } \\
\text { s'additionnent pour représenter le } \\
\text { niveau de référence national. }\end{array}$ & Népal (avril 08) \\
\hline & $\begin{array}{l}\text { Des études approfondies sont } \\
\text { nécessaires pour formuler des } \\
\text { recommandations. }\end{array}$ & USA (avril 08) \\
\hline
\end{tabular}




\begin{tabular}{|c|c|c|}
\hline Questions & Opinions & Appuyées par \\
\hline \multicolumn{3}{|c|}{ Niveaux de référence (suite) } \\
\hline \multirow[t]{2}{*}{$\begin{array}{l}\text { Méthode de } \\
\text { fixation du niveau } \\
\text { de référence } \\
\text { (suite) }\end{array}$} & $\begin{array}{l}\text { Les niveaux de référence historiques } \\
\text { ne sont pas une indication } \\
\text { exacte des pressions futures de } \\
\text { déforestation pour les pays dont } \\
\text { les taux de déboisement actuels } \\
\text { sont bas. }\end{array}$ & $\begin{array}{l}\text { Norvège } \\
\text { (septembre 08) }\end{array}$ \\
\hline & $\begin{array}{l}\text { Utilisation d'un facteur } \\
\text { d'actualisation pour résoudre les } \\
\text { incertitudes liées au niveau de } \\
\text { référence ou prendre en compte les } \\
\text { circonstances nationales. }\end{array}$ & $\begin{array}{l}\text { Greenpeace (mars 08) } \\
\text { PNG (avril 08) }\end{array}$ \\
\hline \multicolumn{3}{|c|}{ Fuite } \\
\hline \multirow[t]{6}{*}{$\begin{array}{l}\text { Comment traiter } \\
\text { les fuites }\end{array}$} & $\begin{array}{l}\text { Suggestion visant à utiliser } \\
\text { l'approche nationale pour prendre } \\
\text { en compte les fuites. }\end{array}$ & $\begin{array}{l}\text { CISDL et GPPI (février } \\
07) \text {; CfRN (septembre } \\
\text { 07) ; CCAP (août 07); } \\
\text { Nouvelle-Zélande } \\
\text { (avril 08) ; FAO, PNUD, } \\
\text { PNUE (avril 08); } \\
\text { Indonésie (avril 08); } \\
\text { USA (avril 08) }\end{array}$ \\
\hline & $\begin{array}{l}\text { Suggestion visant à utiliser d'autres } \\
\text { mécanismes techniques pour traiter } \\
\text { la question des fuites, comme par } \\
\text { ex. de créer des comptes de réserve, } \\
\text { de prendre des assurances et de } \\
\text { limiter les aires de fuites. }\end{array}$ & $\begin{array}{l}\text { Costa Rica (avril 08); } \\
\text { Greenpeace (mars 08); } \\
\text { Colombie (avril 08) }\end{array}$ \\
\hline & $\begin{array}{l}\text { Suggestion visant à instaurer des } \\
\text { mécanismes de réglementation } \\
\text { comme, par ex. l'élimination des } \\
\text { obstacles institutionnels. }\end{array}$ & Indonésie (avril 08) \\
\hline & $\begin{array}{l}\text { Surveillance régulière pour vérifier } \\
\text { les fuites liées à la dégradation. }\end{array}$ & Népal (avril 08) \\
\hline & $\begin{array}{l}\text { Reconnaissance de l'importance de } \\
\text { la question des fuites mais pas de } \\
\text { recommandations spécifiques. }\end{array}$ & $\begin{array}{l}\text { Plan d'Action de } \\
\text { Bali (décembre 07); } \\
\text { CBD (mai 08); USA } \\
\text { (avril 08); Colombie } \\
\text { (avril 08) }\end{array}$ \\
\hline & $\begin{array}{l}\text { Nécessité d'aborder la question } \\
\text { des fuites au niveau international } \\
\text { puisque le problème existe au } \\
\text { niveau des comptes nationaux. }\end{array}$ & $\begin{array}{l}\text { Colombie (avril 08) ; } \\
\text { CCAP (août 07) ; USA } \\
\text { (avril 08) ; Norvège } \\
\text { (septembre 08) }\end{array}$ \\
\hline
\end{tabular}




\begin{tabular}{|c|c|c|}
\hline Questions & Opinions & Appuyées par \\
\hline \multicolumn{3}{|c|}{ Fuite (suite) } \\
\hline \multirow[t]{2}{*}{$\begin{array}{l}\text { Utilisation } \\
\text { des facteurs } \\
\text { d'ajustement }\end{array}$} & $\begin{array}{l}\text { Utiliser les facteurs d'ajustement } \\
\text { pour prendre en compte les fuites. }\end{array}$ & $\begin{array}{l}\text { Greenpeace (mars 08) ; } \\
\text { BioCarbon (Banque } \\
\text { Mondiale août 08) }\end{array}$ \\
\hline & $\begin{array}{l}\text { Il ne faudrait pas utiliser de facteurs } \\
\text { d'ajustement dans la prise en } \\
\text { compte des fuites. }\end{array}$ & $\begin{array}{l}\text { Nouvelle-Zélande } \\
\text { (avril 08) }\end{array}$ \\
\hline \multicolumn{3}{|c|}{ Permanence et responsabilité } \\
\hline \multirow[t]{7}{*}{$\begin{array}{l}\text { Recommandations } \\
\text { concernant la non- } \\
\text { permanence }\end{array}$} & $\begin{array}{l}\text { Utiliser des comptes de réserve pour } \\
\text { prendre en compte la permanence. } \\
\text { Une partie des réductions } \\
\text { d'émissions projetées sont mises } \\
\text { en réserve pour couvrir le risque de } \\
\text { non-permanence. }\end{array}$ & $\begin{array}{l}\text { Costa Rica (avril } \\
08) ; \text { CISDL et GPPI } \\
\text { (février 07) ; Paraguay, } \\
\text { Argentine, Honduras, } \\
\text { Panama, Pérou } \\
\text { (avril 08) }\end{array}$ \\
\hline & $\begin{array}{l}\text { Si des émissions surviennent } \\
\text { pendant la période actuelle, des } \\
\text { crédits carbone pourront être retirés } \\
\text { dans une période future. }\end{array}$ & $\begin{array}{l}\text { Brésil (mars 07) ; PNG } \\
\text { (avril 08) }\end{array}$ \\
\hline & $\begin{array}{l}\text { Création de zones protégées } \\
\text { en tant que moyen d'assurer } \\
\text { la permanence. }\end{array}$ & $\begin{array}{l}\text { CDB (mai 08); Costa } \\
\text { Rica (avril 08) }\end{array}$ \\
\hline & $\begin{array}{l}\text { Utiliser un taux d'actualisation } \\
\text { pour prendre en compte la non- } \\
\text { permanence des crédits REDD. }\end{array}$ & Greenpeace (mars 08) \\
\hline & $\begin{array}{l}\text { Utiliser des crédits temporaires qui } \\
\text { sont renouvelables. }\end{array}$ & $\begin{array}{l}\text { CISDL et GPPI (février } \\
\text { 07) ; BioCarbon } \\
\text { (Banque Mondiale } \\
\text { août 08) }\end{array}$ \\
\hline & $\begin{array}{l}\text { Mettre en place un projet } \\
\text { d'assurances pour gérer les risques } \\
\text { liés aux émissions de } \mathrm{CO}_{2} \text {. }\end{array}$ & $\begin{array}{l}\text { BioCarbon (Banque } \\
\text { Mondiale août 08) } \\
\text { CfRN (septembre 07) }\end{array}$ \\
\hline & $\begin{array}{l}\text { Les pays ne devraient } \\
\text { pas être sanctionnés si la } \\
\text { déforestation est causée par des } \\
\text { catastrophes naturelles. }\end{array}$ & Costa Rica (avril 08) \\
\hline \multirow[t]{2}{*}{$\begin{array}{l}\text { Question de la } \\
\text { responsabilité }\end{array}$} & $\begin{array}{l}\text { Les pays en développement ne } \\
\text { doivent pas être pénalisés s'ils } \\
\text { ne satisfont pas aux objectifs de } \\
\text { réduction d'émissions, mais doivent } \\
\text { bénéficier d'incitations s'ils s'y } \\
\text { conforment. }\end{array}$ & CCAP (août 07) \\
\hline & $\begin{array}{l}\text { Les pays sont tenus pour } \\
\text { responsables du non respect de } \\
\text { leurs obligations. }\end{array}$ & CfRN (septembre 07) \\
\hline
\end{tabular}




\begin{tabular}{|c|c|c|}
\hline Questions & Opinions & Appuyées par \\
\hline \multicolumn{3}{|c|}{ Surveillance, rendre compte et contrôle (MRV) } \\
\hline \multirow[t]{4}{*}{$\begin{array}{l}\text { Surveillance des } \\
\text { émissions liées à } \\
\text { la DD }\end{array}$} & $\begin{array}{l}\text { Les stocks de carbone estimés pour } \\
\text { chaque biome sont associés à des } \\
\text { mesures de télédétection. }\end{array}$ & Brésil (mars 07) \\
\hline & $\begin{array}{l}\text { Télédétection avec vérification } \\
\text { au sol. }\end{array}$ & $\begin{array}{l}\text { UE (avril 08); Népal } \\
\text { (avril 08) }\end{array}$ \\
\hline & $\begin{array}{l}\text { Nécessité de disposer de méthodes } \\
\text { de mesure et de recherche plus } \\
\text { précises. Les stocks de carbone } \\
\text { accumulés dans différents éco- } \\
\text { systèmes sont mal connus. De } \\
\text { très gros investissements sont } \\
\text { nécessaires pour avoir les outils et } \\
\text { méthodes nécessaires. En outre, } \\
\text { nécessité d'étudier des modèles } \\
\text { spécifiques d'écosystèmes. }\end{array}$ & $\begin{array}{l}\text { Chili (août 07), } \\
\text { Paraguay, Argentine, } \\
\text { Honduras, Panama, } \\
\text { Pérou (avril 08) ; USA } \\
\text { (avril 08) }\end{array}$ \\
\hline & $\begin{array}{l}\text { L'approche des stocks de carbone } \\
\text { offre des opportunités aux } \\
\text { pays ayant de faibles taux de } \\
\text { déforestation. 1) Un pays estime } \\
\text { ses stocks de carbone aérien ; 2) } \\
\text { Des « unités de stocks de carbone » } \\
\text { non commercialisables sont } \\
\text { émises égales à (1) ; 3) Une réserve } \\
\text { protégée est établie, protégeant } \\
\text { toutes les forêts qui ne sont pas } \\
\text { en péril contre la déforestation ; } \\
\text { 4) Un projet de conservation pour } \\
\text { une aire boisée située en dehors } \\
\text { de la réserve forestière génère } \\
\text { des crédits ; 5) Au moment de } \\
\text { l'approbation du projet, est créé un } \\
\text { « mécanisme du stock de carbone » } \\
\text { permet tant de commercialiser } \\
\text { les crédits qui seront générés } \\
\text { par le projet ; et 6) les crédits ont } \\
\text { un caractère temporaire afin de } \\
\text { prendre en compte la question de } \\
\text { la permanence. }\end{array}$ & $\begin{array}{l}\text { CISDL et GPPI (février } \\
\text { 07); Sri Lanka (avril } \\
\text { 08); COMIFAC } \\
\text { (mars 07) }\end{array}$ \\
\hline $\begin{array}{l}\text { Incitations au titre } \\
\text { des capacités de } \\
\text { MRV }\end{array}$ & $\begin{array}{l}\text { Reconnaissent le besoin d'un } \\
\text { financement d'avance et } \\
\text { d'incitations pour les activités SRV } \\
\text { et le renforcement des capacités. }\end{array}$ & $\begin{array}{l}\text { Brésil (mars 07); } \\
\text { COMIFAC (avril } \\
\text { 08); Costa Rica } \\
\text { (avril 08); CfRN } \\
\text { (septembre 07); PNG } \\
\text { (avril 08); Norvège } \\
\text { (septembre 08) }\end{array}$ \\
\hline
\end{tabular}




\begin{tabular}{|c|c|c|}
\hline Questions & Opinions & Appuyées par \\
\hline \multicolumn{3}{|c|}{ Surveillance, rendre compte et contrôle (MRV) (suite) } \\
\hline $\begin{array}{l}\text { Approches } \\
\text { en matière de } \\
\text { surveillance }\end{array}$ & $\begin{array}{l}\text { Comptabilisation complète : } \\
\text { Toute la zone forestière du } \\
\text { pays est surveillée - par la } \\
\text { cartographie complète. }\end{array}$ & $\begin{array}{l}\text { Brésil (mars 07) ; } \\
\text { Nouvelle-Zélande } \\
\text { (avril 08) }\end{array}$ \\
\hline $\begin{array}{l}\text { Comptabilisation } \\
\text { complète contre } \\
\text { comptabilisation } \\
\text { partielle }\end{array}$ & $\begin{array}{l}\text { Comptabilisation partielle. } \\
\text { Surveillance par échantillonnage } \\
\text { (systématique ou stratifié) ou } \\
\text { échantillonnage au niveau } \\
\text { du projet. }\end{array}$ & $\begin{array}{l}\text { Indonésie (avril } \\
\text { 08) ; Colombie (avril } \\
08) \text {; COMIFAC (avril } \\
\text { 08) ; Chili (août 07), } \\
\text { Paraguay, Argentine, } \\
\text { Honduras, Panama, } \\
\text { Pérou (avril 08) }\end{array}$ \\
\hline \multirow[t]{2}{*}{$\begin{array}{l}\text { Estimation de } \\
\text { l'efficacité par } \\
\text { rapport aux } \\
\text { réductions des } \\
\text { émissions }\end{array}$} & $\begin{array}{l}\text { L'efficacité des activités adoptées au } \\
\text { titre de la REDD doit être évaluée } \\
\text { par rapport aux montants des } \\
\text { réductions d'émissions obtenues } \\
\text { par le pays hôte, afin d'éviter toute } \\
\text { incitation pernicieuse. }\end{array}$ & Costa Rica (avril 08) \\
\hline & $\begin{array}{l}\text { L'efficacité doit prendre en compte } \\
\text { les effets sur les communautés et } \\
\text { la biodiversité. }\end{array}$ & UE (avril 08) \\
\hline \multirow[t]{3}{*}{$\begin{array}{l}\text { Transparence } \\
\text { des MRV et rôle } \\
\text { des institutions } \\
\text { compétentes }\end{array}$} & $\begin{array}{l}\text { Les données devraient être rendues } \\
\text { publiques et diffusées, par ex., } \\
\text { auprès des COP et sur internet. }\end{array}$ & $\begin{array}{l}\text { Brésil (février 07); } \\
\text { Colombie (avril 08); } \\
\text { Chili (août 07); } \\
\text { Paraguay, Argentine, } \\
\text { Honduras, Panama, } \\
\text { Pérou (avril 08) }\end{array}$ \\
\hline & Rapport annuel sur les inventaires. & $\begin{array}{l}\text { Brésil (mars 07) ; CCAP } \\
\text { (août 07) }\end{array}$ \\
\hline & $\begin{array}{l}\text { Les institutions internationales } \\
\text { faisant partie du GIEC/CCNUCC } \\
\text { définissent les méthodes MRV. }\end{array}$ & $\begin{array}{l}\text { Chili (août 07) ; } \\
\text { Paraguay, Argentine, } \\
\text { Honduras, Panama, } \\
\text { Pérou (avril 08); } \\
\text { Brésil (février 07) ; } \\
\text { Canada (avril 08); } \\
\text { Colombie (avril 08); } \\
\text { Costa Rica (avril 08); } \\
\text { CfRN (septembre } \\
\text { 07) ; OSCTC pour } \\
\text { FAO, PNUD, PNUE } \\
\text { (avril 08) ; Indonésie } \\
\text { (avril 08) ; PNG } \\
\text { (avril 08) ; Norvège } \\
\text { (septembre 08) }\end{array}$ \\
\hline
\end{tabular}




\begin{tabular}{|c|c|c|}
\hline Questions & Opinions & Appuyées par \\
\hline \multicolumn{3}{|c|}{ Surveillance, rendre compte et contrôle (MRV) (suite) } \\
\hline \multirow[t]{2}{*}{$\begin{array}{l}\text { Transparence } \\
\text { des MRV et rôle } \\
\text { des institutions } \\
\text { compétentes } \\
\text { (suite) }\end{array}$} & $\begin{array}{l}\text { Un système de vérification } \\
\text { indépendant et dûment accrédité } \\
\text { est nécessaire pour déterminer les } \\
\text { niveaux de référence des émissions, } \\
\text { les réductions d'émissions réelles et } \\
\text { les fuites. }\end{array}$ & $\begin{array}{l}\text { Colombie (avril } \\
\text { 08) ; Chili (août } \\
\text { 07) ; Paraguay, } \\
\text { Argentine, Honduras, } \\
\text { Panama, Pérou } \\
\text { (avril 08) ; Norvège } \\
\text { (septembre 08) }\end{array}$ \\
\hline & $\begin{array}{l}\text { Les résultats de la surveillance } \\
\text { du carbone ne doivent pas } \\
\text { nécessairement être vérifiés par } \\
\text { des institutions ou des experts } \\
\text { en dehors du pays parce qu'ils } \\
\text { devraient être appuyés par des } \\
\text { mécanismes institutionnels } \\
\text { nationaux adaptés aux } \\
\text { systèmes d'administration des } \\
\text { gouvernements locaux et du } \\
\text { gouvernement central. }\end{array}$ & Indonésie (avril 08) \\
\hline $\begin{array}{l}\text { Mécanisme MRV } \\
\text { Risques liés aux } \\
\text { MRV }\end{array}$ & $\begin{array}{l}\text { Pour éviter les risques, les } \\
\text { réductions d'émissions devraient } \\
\text { être affectées d'un taux } \\
\text { d'actualisation approximatif pour } \\
\text { tenir compte des incertitudes } \\
\text { planant sur les estimations } \\
\text { d'émissions, les niveaux de } \\
\text { référence et la permanence. }\end{array}$ & Greenpeace (mars 08) \\
\hline \multicolumn{3}{|c|}{ Dégradation } \\
\hline \multirow[t]{3}{*}{$\begin{array}{l}\text { Inclure ou exclure } \\
\text { REDD }\end{array}$} & $\begin{array}{l}\text { Inclure les deux, la déforestation et } \\
\text { la dégradation. }\end{array}$ & $\begin{array}{l}\text { Plan d'Action de } \\
\text { Bali (décembre 07); } \\
\text { Norvège (septembre } \\
\text { 08) ; Australie (avril } \\
\text { 08) ; CCAP (août 07); } \\
\text { CISDL et GPPI (février } \\
\text { 07) ; Costa Rica (avril } \\
\text { 08) ; CfRN (septembre } \\
\text { 07) ; UE (avril 08); } \\
\text { Japon (août 08); } \\
\text { Népal (avril 08); USA } \\
\text { (avril 08) }\end{array}$ \\
\hline & $\begin{array}{l}\text { Appuie l'inclusion de la dégradation, } \\
\text { mais pas de façon systématique. } \\
\text { Il appartiendra de décider, dans le } \\
\text { contexte de chaque activité, s'il est } \\
\text { suffisamment rentable d'inclure la } \\
\text { dégradation des forêts. }\end{array}$ & Colombie (avril 08) \\
\hline & $\begin{array}{l}\text { Devrait inclure la dégradation des } \\
\text { forêts, mais en surveillant de près } \\
\text { les questions méthodologiques. }\end{array}$ & Greenpeace (mars 08) \\
\hline
\end{tabular}




\begin{tabular}{|c|c|c|}
\hline Questions & Opinions & Appuyées par \\
\hline \multicolumn{3}{|c|}{ Dégradation (suite) } \\
\hline \multirow[t]{2}{*}{$\begin{array}{l}\text { Inclure ou exclure } \\
\text { REDD (suite) }\end{array}$} & $\begin{array}{l}\text { Opposé à l'inclusion de la } \\
\text { dégradation des forêts, car cela } \\
\text { met en péril le processus de } \\
\text { la REDD. Certaines questions } \\
\text { méthodologiques nécessaires pour } \\
\text { mesurer la dégradation ne sont } \\
\text { pas résolues. }\end{array}$ & Brésil (mars 07) \\
\hline & $\begin{array}{l}\text { REDD d'abord, REDD partout où il } \\
\text { est possible de l'appliquer, ou plus } \\
\text { tard si l'appliquer est réalisable. } \\
\text { Les pays qui ne sont pas prêts - au } \\
\text { motif qu'ils ne disposent pas des } \\
\text { méthodologies nécessaires pour } \\
\text { mesurer la dégradation des forêts } \\
\text { - devraient pouvoir bénéficier } \\
\text { d'incitations pour réduire la } \\
\text { déforestation. }\end{array}$ & $\begin{array}{l}\text { Canada (avril 08); UE } \\
\text { (avril 08); Greenpeace } \\
\text { (mars 08) }\end{array}$ \\
\hline $\begin{array}{l}\text { REDD ou RED } \\
\text { (incluant ou } \\
\text { excluant la } \\
\text { dégradation) } \\
\text { (suite) }\end{array}$ & $\begin{array}{l}\text { Nos difficultés en matière de } \\
\text { méthodologies à appliquer pour } \\
\text { mesurer la dégradation peuvent } \\
\text { être surmontées et nous devrions } \\
\text { pouvoir inclure la dégradation des } \\
\text { forêts sans plus tarder. }\end{array}$ & Costa Rica (avril 08) \\
\hline \multirow[t]{2}{*}{$\begin{array}{l}\text { Surveillance de la } \\
\text { dégradation }\end{array}$} & $\begin{array}{l}\text { Un certain nombre de questions } \\
\text { liées à la surveillance restent en } \\
\text { suspens. Les recherches n'ont pas } \\
\text { montré que des changements } \\
\text { marginaux associés à la dégradation } \\
\text { des forêts puissent être décelés } \\
\text { avec précision seulement par } \\
\text { télédétection. Des méthodes } \\
\text { d'enquêtes répétées par } \\
\text { échantillonnage sont nécessaires } \\
\text { pour déterminer les flux nets de ce } \\
\text { type d'activités. }\end{array}$ & USA (avril 08) \\
\hline & $\begin{array}{l}\text { Une approche indirecte a été } \\
\text { suggérée pour surveiller la } \\
\text { dégradation des forêts (zones de } \\
\text { forêts intactes et non intactes entre } \\
\text { deux périodes d'observation). } \\
\text { Au nombre des méthodes pour } \\
\text { estimer et démontrer les réductions } \\
\text { d'émissions dues à la dégradation } \\
\text { figurent notamment : les inventaires } \\
\text { ou l'évaluation des trouées dans } \\
\text { la canopée par télédétection avec } \\
\text { vérification par des mesures au sol. }\end{array}$ & $\begin{array}{l}\text { UE (avril 08); } \\
\text { Colombie (avril 08); } \\
\text { Népal (avril 08) }\end{array}$ \\
\hline
\end{tabular}




\begin{tabular}{|c|c|c|}
\hline Questions & Opinions & Appuyées par \\
\hline \multicolumn{3}{|c|}{ Dégradation (suite) } \\
\hline \multirow[t]{4}{*}{$\begin{array}{l}\text { Conséquences de } \\
\text { l'inclusion de la } \\
\text { dégradation }\end{array}$} & $\begin{array}{l}\text { Des ressources supplémentaires } \\
\text { sont nécessaires pour mesurer la } \\
\text { dégradation, notamment davantage } \\
\text { d'enquêtes par échantillonnage et } \\
\text { de surveillance sur le terrain, et des } \\
\text { compléments de financement pour } \\
\text { le renforcement des capacités et la } \\
\text { mise en œuvre. }\end{array}$ & $\begin{array}{l}\text { CCAP (août } \\
\text { 07) ; Norvège } \\
\text { (septembre 08) }\end{array}$ \\
\hline & $\begin{array}{l}\text { La réduction de la dégradation } \\
\text { entraînera un certain nombre de } \\
\text { bénéfices associés, notamment sur } \\
\text { le plan de la biodiversité. }\end{array}$ & $\begin{array}{l}\text { CISDL et GPPI } \\
\text { (février 07) }\end{array}$ \\
\hline & $\begin{array}{l}\text { Il convient de revoir les définitions } \\
\text { existantes pour la déforestation et la } \\
\text { dégradation des forêts. }\end{array}$ & $\begin{array}{l}\text { Colombie (avril 08); } \\
\text { COMIFAC (avril 08) }\end{array}$ \\
\hline & $\begin{array}{l}\text { Une gestion intégrée des tourbières } \\
\text { doit être mise en place. La } \\
\text { dégradation à grande échelle des } \\
\text { tourbières, y compris des tourbières } \\
\text { boisées, a des effets importants } \\
\text { sur le changement climatique, la } \\
\text { biodiversité et les populations. }\end{array}$ & CDB (mai 08) \\
\hline \multicolumn{3}{|c|}{ Bénéfices associés à la REDD } \\
\hline \multirow[t]{4}{*}{$\begin{array}{l}\text { Positions diverses } \\
\text { en ce qui concerne } \\
\text { les bénéfices } \\
\text { associés à la REDD }\end{array}$} & $\begin{array}{l}\text { La préservation de l'environnement } \\
\text { et les bénéfices associés à la } \\
\text { biodiversité sont essentiels pour } \\
\text { assurer une bonne efficacité. }\end{array}$ & $\begin{array}{l}\text { Plan d'Action de } \\
\text { Bali (décembre } \\
\text { 07) ; CDB (mai 08); } \\
\text { FAO-PNUD-PNUE, } \\
\text { Greenpeace (mars 08) ; } \\
\text { BioCarbon (Banque } \\
\text { Mondiale août 08); } \\
\text { Mexique (août 08) ; UE } \\
\text { (juillet 08) }\end{array}$ \\
\hline & $\begin{array}{l}\text { Les objectifs concernant la } \\
\text { réduction de la pauvreté et le } \\
\text { développement durable devraient } \\
\text { être inclus dans tout projet REDD. }\end{array}$ & $\begin{array}{l}\text { FAO-PNUD-PNUE, } \\
\text { BioCarbon (Banque } \\
\text { Mondiale août 08); } \\
\text { Mexique (août 08) }\end{array}$ \\
\hline & $\begin{array}{l}\text { Il faut assurer la protection des } \\
\text { droits des groupes minoritaires, des } \\
\text { communautés dépendantes des } \\
\text { forêts et des groupes indigènes. }\end{array}$ & $\begin{array}{l}\text { FAO-PNUD-PNUE, } \\
\text { Greenpeace (mars } \\
\text { 08) ; UE (juillet } \\
\text { 08) ; Norvège } \\
\text { (septembre 08) }\end{array}$ \\
\hline & $\begin{array}{l}\text { Les bénéfices associés ne devraient } \\
\text { pas avoir d'influence sur la mise en } \\
\text { œuvre de la REDD. }\end{array}$ & PNG (avril 08) \\
\hline
\end{tabular}




\section{Abréviations}

3E

Efficacité, Efficient, et Equitable (Effectiveness, Efficiency and

Equity)

B/R

Boisement/ Reboisement (Afforestation/Reforestation)

AAU

Unités de quantité attribuée/Assigned Amount Unit

$\mathrm{AD}$

Déforestation évitée (Avoided deforestation)

AFOLU

Agriculture, foresterie et autres affectations des terres

(Agriculture, Forestry and other Land Use)

ARD

Boisement, reboisement et déboisement (Afforestation, Reforestation, and Deforestation)

BAU Scénario de la poursuite de la tendance actuelle ou scénario BAU (Business as Usual)

CAT plafonnement et échange (Cap and Trade)

$\mathrm{CDB} / \mathrm{CBD} \quad$ Convention sur la diversité biologique (Convention on

Biodiversity)

CCAP Center for Clean Air Policy

MDP/CDM Mécanisme pour un développement propre (Clean Development Mechanism)

REC/CER Réduction des émissions certifiée (Certified Emission Reduction)

CfRN Coalition for Rainforest Nations

CIFOR Centre de recherche forestière internationale (Center for

International Forestry Research)

CISDL Centre for International Sustainable Development Law

e $-\mathrm{CO}_{2} \quad$ équivalent-dioxyde de carbone

COP/CdP Conférence des Parties

DD

Déforestation et dégradation des forêts

DNA Designated National Authority/Autorité nationale désignée

ETS Emissions Trading Scheme/Système d'échange de quotas

d'émissions de GES

UE Union Européenne 
FEM/GEF Fonds pour l'Environnement mondial/ Global Environmental Facility

GES/GHG Gaz à effet de serre/Greenhouse Gases

GOFC - GOLD Global Observation of Forest and Land Cover Dynamics

GBP/GPG Guide de bonnes pratiques/ Good Practice Guide

GPPI

ha

Global Public Policy Institute

ICDP Integrated Conservation and Development Project (Projet intégré de conservation et de développement)

GIEC/IPCC Groupe d'experts intergouvernemental sur l'évolution du climat/ Intergovernmental Panel on Climate Change

MOC/JI Mise en œuvre conjointe/ Joint Implementation

PMA/LDC Pays les moins avancés/ Less Developed Countries

LULUCF Land Use, Land Use Change, Forestry/ utilisation des terres, changements d'affectation des terres et foresterie

MRV Monitoring, Reporting, and Verification (surveillance, suivi et contrôle)

APD/ODA Aide publique au développement/ Official Development Assistance

PM Politiques et mesures

PSE/PES Paiements pour services environnementaux/ Payments for Environmental Services

PNG Papouasie Nouvelle Guinée

REDD Réduction des émissions dues à la déforestation et à la dégradation des forêts/ Reducing Emissions from Deforestation and Degradation

OSCST/SBSTA Organe subsidiaire de conseil scientifique et technologique/ Subsidiary Body for Scientific and Technological Advice

TDERM Tropical Deforestation Emission Reduction Mechanism

$\mathrm{tC}$ tonnes métriques de carbone

$\mathrm{TgC}$

Téragrammes de carbone $=$ Millions de tonnes métriques de carbone

CCD/ UNCCD Convention contre la désertification (Convention des Nations Unies sur la lutte contre la désertification)/ United Nations Convention to Combat Desertification

FNUF/UNFF United Nations Forum on Forests/Forum des Nations Unies sur les forêts

CCNUCC/ Convention-cadre des Nations Unies sur les changements

UNFCCC climatiques/ United Nations Framework Convention on Climate Change

REV/VER Réduction d'émissions vérifiée/ Verified Emission Reduction 


\section{Glossaire}

Abattage à impact réduit/ Reduced impact logging (RIL)

Exploitation intensive planifiée et contrôlée avec soin pour réduire au minimum l'impact de l'abattage sur les sols et parcelles de forêts restantes, habituellement en sélectionnant les arbres à abattre un par un.

\section{Additionnalité}

Se réfere à la nature des projets visés par le Protocole de Kyoto. Ces projets doivent faire la preuve de leur " additionnalité " - c'est à dire qu'ils ont pour résultat des bénéfices réels et mesurables sur une longue période de temps, pour réduire ou prévenir les émissions de carbone qui se seraient produites en l'absence de ces projets. Par ailleurs, l'additionnalité au sens des systèmes de comptabilisation s'entend de paiements effectués au titre d'émissions qui ont été réduites à un niveau inférieur à celui du scénario de la poursuite de la tendance actuelle ou scénario BAU (voir sous le terme business-as-usual BAU).

\section{AFOLU}

Acronyme pour Agriculture, Forestry and Other Land Uses. Recommandé dans les lignes directrices du GIEC (2006) comme terme nouveau pour englober les activités LULUCF (Land Use, Land Use Change and Forestry) et l'agriculture.

\section{Atténuation/Mitigation}

Actions visant à prévenir une plus grande accumulation de GES dans l'atmosphère en réduisant les quantités émises ou en augmentant le stockage dans les puits de carbone.

\section{Approche combinéel Nested approach}

Approche hybride qui inclue des aspects relevant des deux autres approches de la REDD aux niveaux sous-national et national. Voir le chapitre 4 pour une description plus détaillée.

\section{Bénéfices associés/Co-benefits}

Bénéfices associés aux projets REDD (autres que ceux qui résultent de la réduction des émissions de GES) comme la réduction de la pauvreté, la protection de l'environnement, l'amélioration de la biodiversité, le renforcement de la gouvernance forestière et la protection des droits humains. 


\section{BioCarbon Fund}

Un fonds public/privé administré par la Banque Mondiale pour faire la démonstration de projets qui séquestrent ou stockent le carbone dans les forêts et les agrosystèmes.

\section{Biomasse}

Le poids sec de toute la matière organique vivante.

\section{Boisement/ Afforestation}

Le Protocole de Kyoto définit le boisement (Afforestation) comme la conversion directe anthropique des terres non forestières en terres forestières permanentes (pendant une période d'au moins 50 ans).

\section{Coalition for Rainforest Nations (CfRN)}

Initiative de collaboration entre pays en développement dotés de forêts tropicales pour concilier gestion et surveillance forestières (forest stewardship) et développement économique. En novembre 2008, la coalition comprenait 41 pays de quatre continents (Asie, Afrique, Amériques et Océanie. La Coalition agit parfois comme un seul groupe aux réunions de la CCNUCC. Elle est l'auteur d'un certain nombre de communications au titre de la REDD.

\section{Conférence des Parties (COP)}

C'est le conseil d'administration qui se réunit une fois par an.

\section{Couvert du houppier/ Crown cover}

C'est le pourcentage de la surface d'un écosystème situé sous la canopée d'un arbre. On se réfère aussi au couvert de la canopée ou simplement au couvert boisé.

\section{Couverture de la canopéel Canopy cover}

Voir « crown cover".

\section{Critère des 3E}

Le critère des $3 \mathrm{E}$ (efficacité, efficience et équité) a tout d'abord été utilisé dans le Rapport Stern (voir le terme Rapport Stern plus loin) pour évaluer les projets de réduction des émissions globales de gaz à effet de serre (GES). Ces critères sont repris dans ce livre pour évaluer différentes options d'architecture globale pouvant s'appliquer dans le cadre du programme REDD (voir fin du chapitre 2 pour explications plus détaillées).

\section{Déforestation}

Dans la plupart des définitions, on décrit la déforestation comme la conversion à longue échéance ou de façon permanente de terres forestières en terres non forestières. Dans une annexe à une décision prise par la Conférence des Parties (CdP) de la CCNUCC agissant comme réunion des Parties au Protocole de Kyoto, la déforestation est définie comme " la conversion anthropique directe de terres forestières en terres non forestières ». La FAO définit la déforestation comme « la conversion de la 
forêt à une autre affectation ou la réduction de longue durée du couvert du houppier au-dessous du seuil minimum de 10 pour cent ». D'autres définitions stipulent aussi la hauteur minimale des arbres (FAO : $5 \mathrm{~m}$ in situ) et les superficies minimales (FAO :0,5 ha), indiquant en outre que l'agriculture ne doit pas constituer l'activité dominante affectée à ces terres. Mais les définitions concernant le couvert du houppier, la hauteur des arbres et la superficie minimale varient d'un pays à l'autre.

\section{Dégradation}

Changements qui surviennent dans la forêt et affectent négativement la structure ou la fonction d'un peuplement ou d'un site forestier et, par conséquent, diminuent la capacité de la forêt à fournir des produits et/ ou des services. Dans le contexte du mécanisme REDD, la dégradation de la forêt entraîne une perte nette de carbone pour l'écosystème. Une façon de mesurer la dégradation consiste à mesurer la diminution $\mathrm{du}$ stock de carbone par unité de superficie (par exemple, par hectare).

\section{Droits sur le carbone/ Carbon rights}

Les droits sur le carbone s'entendent des revendications portant sur les bénéfices provenant de réservoirs de carbone, par exemple d'une parcelle forestière spécifique. Quand existe un marché des réductions d'émissions de GES, les droits sur le carbone peuvent avoir une valeur financière. Ces droits peuvent aussi désigner les responsabilités de gestion associées à une zone forestière spécifique. Les questions relevant des droits sur le carbone comportent la définition de ces droits et la façon dont ils sont administrés dans des zones où les régimes fonciers ne sont pas clairement établis et où les institutions en place n'ont pas les moyens suffisants pour faire appliquer la législation.

\section{Fongibilité (ou compatibilité) (des droits d'émission ou crédits REDD)}

C'est le degré d'interchangeabilité entre les crédits (ou droits d'émission) REDD et les crédits carbone sur les marchés du carbone. Quand les crédits REDD sont entièrement fongibles (ou compatibles), ils peuvent être vendus sans restriction et utilisés à des fins comme celles visant à atteindre les objectifs de réduction d'émissions dans les pays qui s'y sont engagés.

\section{Fuite inversée/ Reverse leakage}

Activité d'atténuation qui entraîne des réductions d'émissions dans des zones situées à l'extérieur de la zone d'atténuation initiale. Elle est aussi qualifiée de "fuite positive".

\section{Fuite/ Leakage}

Dans le contexte du changement climatique, les fuites de carbone sont le résultat d'interventions qui - engagées dans une zone géographique donnée (au niveau sous-national ou national) pour réduire les émissions - entrainent une augmentation des émissions dans une autre zone. On entend par « fuite », par exemple, le processus selon lequel le 
fait d'empêcher l'empiètement de l'agriculture dans les zones forestières d'une région a pour résultat la conversion de zones forestières en terres agricoles dans une autre région. Dans le contexte de la REDD, on qualifie aussi ce phénomène de fuite comme un "déplacement des émissions ".

\section{LULUCF}

Acronyme pour Land Use, Land-Use Change and Forestry (utilisation des terres, changements d'affectation des terres et foresterie). Voir aussi AFOLU.

\section{Marchés du carbone/ Carbon Markets}

Tout marché dans lequel se déroulent des transactions commerciales liées aux émissions de carbone, habituellement sous forme d'octroi de droits d'émission du carbone (ou crédits carbone). Il y a deux catégories de marchés du carbone : les marchés volontaires (dans lesquels les objectifs de réduction d'émissions ne sont pas réglementés) et les marchés réglementaires (dans lesquels les droits d'émission ou crédits carbone font l'objet d'échanges satisfaisant aux objectifs réglementaires fixés en matière de réduction d'émissions). Le marché du carbone le plus important actuellement est le système de quotas d'émissions (ETS) de l'UE.

Mécanisme pour un développement propre (MDP)/Clean Development Mechanism (CDM)

Il s'agit d'un mécanisme établi au titre du Protocole de Kyoto pour aider les pays développés (Annexe I) à se conformer à leurs objectifs en matière d'émissions. Ce mécanisme prévoit que des droits (ou crédits) d'émission seront accordés aux pays visés à l'Annexe I qui financent et mettent en œuvre des projets réduisant les émissions dans les pays en développement (non visés à l'Annexe I) et que ces crédits pourront être utilisés afin de satisfaire aux objectifs fixés en matière de réduction d'émissions aux pays de l'Annexe I. Le CDM ne vise pas seulement à réduire les émissions ou à augmenter les puits de carbone, mais aussi à contribuer au développement durable dans les pays en développement.

Mise en œuvre conjointe (MOC)/ Joint Implementation (JI)

Mécanisme établi au titre du Protocole de Kyoto (parallèlement à celui du MDP) afin d'aider les pays visés à l'Annexe I à atteindre leurs objectifs de réduction d'émissions en investissant dans des projets de réduction d'émissions dans d'autres pays développés en tant qu'alternative à une réduction de leurs émissions sur le territoire national. Contrairement au MDP, les réductions d'émissions au titre de la MOC ont lieu dans des pays qui se sont fixés des objectifs de réduction d'émissions GES.

\section{Niveau de référence/ Baseline}

Le niveau de référence peut se référer à trois concepts (voir chapitre 6) : (i) le niveau de référence historique, c.-à-d. le taux de déforestation et de 
dégradation des forêts et les émissions de $\mathrm{CO}_{2}$ en résultant pendant les $\mathrm{x}$ années passées ; (ii) le taux de déforestation et dégradation projeté dans un scénario du laisser-faire (BAU). Un niveau de référence BAU est le point de repère à partir duquel juger de l'impact des mesures REDD et veiller à l'additionnalité ; et (iii) le niveau de référence pour l'octroi de crédits carbone (ou droits d'émission) est le point de repère à partir duquel récompenser un pays (ou projet) si les émissions sont inférieures à ce niveau.

\section{Niveau/base de référence/ Reference level/line}

Communément utilisé dans le sens de crediting baseline/niveau de référence de comptabilisation (voir "baseline " / " niveau de référence ").

\section{Normes volontaires en matière de carbone/ Voluntary Carbon Standards}

Projets de certification pour des crédits (droits) d'émission non réglementés au titre du Protocole de Kyoto.

\section{Paiements basés sur les intrants/Input-based payments}

Paiements effectués à condition que soient fournis les intrants qui sont censés entraîner des réductions d'émissions dont les résultats ne peuvent cependant pas être directement mesurés (ou à des conditions très onéreuses). On se réfère souvent à ces projets (paiements basés sur les intrants) sous le titre de " politiques et mesures " (PM).

\section{Paiements basés sur les résultats/ Output-based payments}

Les paiements basés sur les résultats sont des paiements directement conditionnés par les résultats. Deux catégories de mesures de ce type sont actuellement étudiées dans le cadre du débat sur la REDD : les mesures basées sur les émissions et les mesures basées sur les stocks. La première approche, basée sur les émissions (ou les flux d'émissions) ne mesure et comptabilise que les variations nettes des stocks de carbone au fil du temps (autrement dit, les émissions). L'approche basée sur les stocks fait des paiements une fonction du stock total de carbone dans une forêt à un moment donné (c'est-à-dire que ce sont les niveaux absolus qui sont pris en compte et non les variations).

\section{Paiements pour les services (écosystèmes) environnementaux (PSE)}

Paiement volontaire effectué par (au moins) un acheteur à (au moins) un fournisseur pour " acheter» un service environnemental (ou l'utilisation d'une terre susceptible de rendre ce service), à la condition expresse que le fournisseur garantisse le service environnemental.

Pays visés et pays non visés à l'Annexe I/ Annee I and Non-Annee I Countries Au titre de la Convention-cadre des Nations Unies sur le changement climatique (CCNUCC), les nations relèvent de deux catégories : 
les pays développés (dénommés " pays visés à l'Annexe I ») et les pays en développement (dénommés " pays non visés à l'Annexe I "). Conformément au principe des responsabilités communes mais différenciées, les pays visés à l'Annexe I ont de plus hauts niveaux d'engagement en matière de mise en vigueur et de rapports de suivi des stratégies qu'ils appliquent et la plupart ont pris des engagements de réduction de leurs émissions au titre du Protocole de Kyoto.

\section{Permanence}

Se réfère à la durée et à l'irréversibilité d'une réduction d'émissions de GES. La non-permanence peut être considérée comme une forme de fuite dans le temps.

\section{Protocole de Kyoto}

Accord conclu en 1997 au titre de la Convention cadre des Nations Unies sur les changements climatiques (CCNUCC). Visés à l'Annexe I, les pays (qualifiés de "pays de l'Annexe I») qui ratifient ce Protocole s'engagent à réduire leurs émissions de dioxyde de carbone et de cinq autres GES. Le Protocole de Kyoto recouvre maintenant plus de 170 pays dans le monde mais seulement 60 pour cent des quantités globales d'émissions liées aux GES. Au mois de décembre 2007, les Etats Unis et le Kazakhstan étaient les deux seuls pays n'ayant pas ratifié le Protocole. La première période d'engagement du Protocole de Kyoto se termine en 2012 et les négociations internationales ont repris en mai 2007 pour débattre de la prochaine période d'engagement.

\section{Rapport Stern/ Stern Review}

Le Rapport Stern (Stern Review on the Economics of Climate Change) est un rapport de 700 pages commandité par le gouvernement du Royaume Uni. Etabli par l'éminent économiste, Lord Stern of Brentford, et paru le 30 octobre 2006, le rapport étudie les effets du changement et du réchauffement climatiques sur l'économie mondiale. Il conclut qu'il faut investir 1 (un) pour cent du produit intérieur brut (PIB) mondial par an pour éviter les effets les plus néfastes du changement climatique. $\mathrm{Ne}$ pas le faire équivaudrait à prendre le risque de faire chuter le PIB mondial de 20 pour cent.

\section{Plan d'Action de Bali/ Bali Action Plan}

Le Plan d'Action de Bali se réfère à la Décision 1/CP.13 qui établit le cadre des négociations internationales pour lancer « ...un vaste processus pour permettre l'application intégrale, effective et continue de la Convention par une action concertée à long terme, dès à présent, d'ici à 2012 et au-delà ". Le Plan d'Action inclut des dispositions sur " des démarches générales et des mesures d'incitation positives pour tout ce qui concerne la réduction des émissions résultant du déboisement et de la dégradation des forêts dans les pays en développement ; ainsi que le rôle de la préservation et du développement durable des forêts 
et du renforcement des stocks de carbone forestiers dans les pays en développement ".

\section{Puits de carbone/ Carbon sink}

Un réservoir qui absorbe ou reprend du carbone libéré par d'autres composants du cycle du carbone

\section{Reboisement/ Reforestation}

Le reboisement est " la conversion anthropique directe de terres forestières en terres non forestières à travers des activités humaines de plantation, d'ensemencement et/ou de promotion de sources naturelles d'ensemencement sur des terres antérieurement boisées mais qui ont été converties en terres non boisées ». Pendant la première période d'engagement du Protocole de Kyoto, les activités de reboisement ont été définies comme le reboisement de superficies qui n'étaient pas boisées au 31 décembre 1989 mais l'avaient été à un certain moment dans le courant des 50 dernières années.

\section{Réduction des émissions dues (liées) à la déforestation et à la dégradation des forêts (REDD)}

Par REDD on entend les mécanismes faisant actuellement l'objet de négociations dans le cadre du processus de la CCNUCC pour réduire les émissions dues à la déforestation et à la dégradation des forêts. La REDD peut se référer à un vaste ensemble d'approches et d'actions qui devraient précisément permettre d'atteindre cet objectif, mais l'idée essentielle est de créer des mécanismes basés sur la performance qui récompenseront des projets ou des pays qui réussiront à réduire les émissions.

\section{Réductions fictives}

Réductions d'émissions qui ne sont pas vraiment additionnelles (voir "additionnalité »). A titre d'illustration, il y a lieu de se reporter à la situation qui prévalait dans l'ex-Union Soviétique et en Europe de l'Est. Le déclin de l'économie dans les années 1990 a entraîné une forte diminution des émissions de GES. Les niveaux d'émission étaient inférieurs à ceux de 1990 qui avaient été pris comme référence de base de comptabilisation. Conformément aux règles du Protocole de Kyoto, ces pays avaient le droit de vendre la différence en tant que droits d'émission malgré le fait que ces crédits leur étaient accordés au titre de réductions qui se seraient, de toutes façons, produites (c.-à-d., non additionnelles).

\section{Rente forestière/ Forest rent}

La rente forestière peut être définie comme le profit net tiré d'une parcelle de terre forestière, c'est à dire la différence entre le revenu brut provenant des produits et services résultant des diverses activités engagées et les coûts d'opportunité des facteurs de production (intrants) utilisés. 
Réservoir de carbone/ Carbon pool

Cette expression désigne un réservoir ou stock qui a la capacité d'accumuler ou de libérer du carbone. Dans les forêts, il y a cinq principaux réservoirs de carbone : la biomasse aérienne, la biomasse souterraine, la litière, le bois mort et le carbone organique des sols.

\section{Responsabilitél Liability}

Il s'agit de l'obligation à laquelle sont tenus les pays ou les projets qui mettent en œuvre la REDD de veiller à la permanence des crédits accordés au titre des réductions d'émissions. Ce terme est essentiellement utilisé dans le contexte de la permanence de la REDD This terms is mainly used in relation to the permanence of REDD (Mainly used in relation to the permanence of REDD).

\section{Revégétation}

La repousse d'une végétation nouvelle dans une zone antérieurement défrichée.

Scénario de la poursuite de la tendance actuelle/ Business-as-Usual (BAU) Une référence politiquement neutre aux émissions futures, c'est à dire aux niveaux futurs d'émission en l'absence d'activités de REDD.

\section{Séquestration du carbone/ Carbon sequestration}

Le retrait du carbone de l'atmosphère et le stockage à long terme dans les puits, comme les océans ou les écosystèmes terrestres, à travers des processus physiques ou biologiques comme la photosynthèse.

Stocks de carbone/ Carbon stocks

La quantité de carbone contenue dans un réservoir de carbone (voir " réservoir de carbone ").

\section{Télédétection/ Remote sensing}

Méthode utilisée pour mesurer le degré de déforestation et/ou de dégradation de la forêt avec un appareil enregistreur qui n'est pas matériellement en contact avec la forêt, comme un satellite par exemple.

\section{Tourbières}

Zones humides (Wetlands) dont les sols sont riches en matières organiques parce qu'ils sont essentiellement formés de végétaux en partie décomposés.

\section{Transition des forêts/ Forest transition}

Description des variations du couvert forestier dans le temps en fonction des quatre stades suivants : (i) couvert boisé initialement élevé et faible déforestation ; (ii) déboisement accéléré et taux de déforestation élevé ; (iii) ralentissement de la déforestation et stabilisation du couvert boisé ; et (vi) période de reboisement. 
Unité de réduction certifiée des émissions (URCE)/Certified Emission Reduction (CER)

C'est le terme technique pour désigner le résultat des projets MDP. Une URCE est une unité de réduction d'émissions de GES qui a été certifiée au titre des dispositions de l'article 12 du Protocole de Kyoto, le mécanisme pour un développement propre. Une unité de réduction certifiée (URCE) vaut une tonne de carbone. Deux catégories spécifiques d'URCE peuvent être délivrées pour des activités de boisement et reboisement $(\mathrm{B} / \mathrm{R})$ au titre du MDP : (i) des réductions temporaires certifiées d'émissions (URCE-T) ; et (ii) des réductions certifiées d'émissions de longue durée (URCE-LD)

\section{Vérification}

Evaluation conduite par une tierce partie indépendante sur les réductions d'émissions réelles ou attendues correspondant à une activité d'atténuation particulière.

\section{Zones humides/ Wetlands}

Terres à la jonction des écosystèmes terrestres et aquatiques. Elles sont caractérisées par la présence d'eaux permanentes pendant au moins une partie de l'année. 



\section{Bibliographie}

Achard, F., Belward, A.S., Eva, H.D., Federici, S., Mollicone, D. et Raes, F. 2005 Accounting for avoided conversion of intact and non-intact forests. Technical options and a proposal for a policy tool. Centre commun de recherche de la Commission Européenne.

Achard, F., DeFries, R., Herold, M., Mollicone, D., Pandey, D. et de Souza, C. 2008 Guidance on monitoring of gross changes in forest area. Chapitre 3. Dans: GOFC-GOLD. Reducing greenhouse gas emissions from deforestation and degradation in developing countries: A sourcebook of methods and procedures for monitoring, measuring and reporting. GOFCGOLD Report version COP 13-2. GOFC-GOLD Project Office, Natural Resources Canada, Alberta, Canada.

Alvarado, L., Rubio, X. et Wertz-Kanounnikoff, S. 2007 Why are we seeing” REDD'? An analysis of the international debate on reducing emissions from deforestation and degradation in developing countries. Institut du Développement Durable et des Relations Internationales (IDDRI), Paris.

Angelsen, A. et Kaimowitz, D. 1999 Rethinking the causes of deforestation: Lessons from economic models. World Bank Research Observer 14 (1): 73-98.

Angelsen, A. 2007 Forest cover change in space and time: Combining von Thünen and the forest transition. World Bank Policy Research Working Paper 4117. Banque Mondiale, Washington, D.C.

Anger, N. et Sathaye, J. 2008 Reducing deforestation and trading emissions: Economic implications for the post-Kyoto market. Discussion Paper No. 08016. Center for European Economic Research, Mannheim, Allemagne.

Asner, G.P., Knapp, D.E., Broadbent, E.N., Oliveira, P.J.C., Keller, M. et Silva, J.N. 2005 Selective logging in the Brazilian Amazon. Science 310 (5747): 480-482.

Aukland, L., Costa, P.M. et Brown, S. 2003 A conceptual framework and its application for addressing leakage: The case of avoided deforestation. Climate Policy 3 (2): 123-136.

Blanco, J. et Forner, C. 2000 Special considerations regarding the 'expiring CER's " proposal. International Forum on Enhancement of Japan's Private Sector's Overseas Re-afforestation Cooperation. Ministerio del Medio Ambiente de Colombia, Bogotá, Colombie. 
Börner, J. et Wunder, S. 2008 Paying for avoided deforestation in the Brazilian Amazon: From cost assessment to scheme design. International Forestry Review 10 (3): 496-511.

Balmford, A. et Whitten, T. 2003 Who should pay for tropical conservation, and how could the costs be met? Oryx 37 (2): 238-250.

Banque Mondiale 2008 Climate investment funds: Mapping of existing and emerging sources of forest financing (CIF/FDM.1/2, October 7, 2008). First design meeting on the forest investment program, Washington, D.C., 16-17 octobre 2008.

http://siteresources.worldbank.org/INTCC/Resources/Mapping_study_ Final_for_FIP_Design_Meeting_Oct_16-17_08.pdf (25 nov. 2008).

Banque Mondiale 2004 Sustaining forests: A development strategy. Banque Mondiale, Washington, D.C. http://siteresources.worldbank.org/ INTFORESTS/Resources/SustainingForests.pdf (25 nov. 2008).

Brown, D. et Peskett, L. 2008 International forest policy: Integrated climate and forestry policy options. Policy Department A: Economic and Scientific Policy, DG Internal Policies, Parlement Européen, Bruxelles.

Brown, K., Adger, W.N., Boyd, E., Corbera-Elizalde, E. et Shackley, S. 2004 How do CDM projects contribute to sustainable development? Tyndall Centre Technical Report No. 16. Tyndall Centre, Norwich, RU. http:// www.tyndall.ac.uk/research/theme2/final_reports/it1_13.pdf $(25$ nov. 2008).

Brown, S., Hall, M., Andrasko, K., Ruiz, F., Marzoli, W., Guerrero, G., Masera, O., Dushku, A., de Jong, B. et Cornell, J. 2007 Baselines for landuse change in the tropics: Application to avoided deforestation projects. Mitigation and Adaptation Strategies for Global Change 12 (6): 1001 1026.

Brown, S. et Braatz, B. 2008 Methods for estimating $\mathrm{CO}_{2}$ emissions from deforestation and forest degradation. Chapitre 5. Dans: GOFC-GOLD. Reducing greenhouse gas emissions from deforestation and degradation in developing countries: a sourcebook of methods and procedures for monitoring, measuring and reporting. GOFC-GOLD Report version COP 13-2. GOFC-GOLD Project Office, Natural Resources Canada, Alberta, Canada.

Bruijnzeel, L.A. 2004 Hydrological functions of tropical forests: not seeing the soil for the trees? Agriculture, Ecosystems \& Environment 104 (1): $185-228$.

Byron, N. et Arnold, M. 1999 What future for the peoples of the tropical forests? World Development 27 (5):789-805.

CE (Commission Européenne) 2008 Addressing the challenges of deforestation and forest degradation to tackle climate change and biodiversity loss. Communication de la Commission au Parlement Européen, Le Conseil, Le Comité économique et social européen et le Comité des régions. Com (2008) 645/3. Bruxelles. 
Chave, J., Andalo, C., Brown, S., Cairns, M.A., Chambers, J.Q., Eamus, D., Fölster, H., Fromard, F., Higuchi, N., Kira, T., Lescure, J.P., Nelson, B.W., Ogawa, H., Puig, H., Riéra, B. et Yamakura, T. 2005 Tree allometry and improved estimation of carbon stocks and balance in tropical forests. Oecologia 145 (1): 87-99.

Chomitz, K.M. 2000 Evaluating carbon offsets from forestry and energy projects: How do they compare? World Bank Policy Research Working Paper No. 2357. Banque Mondiale, Washington, D.C.

Chomitz, K.M., Buys, P., de Luca, G., Thomas, T.S. et Wertz-Kanounnikoff, S. 2006 At loggerheads? Agricultural expansion, poverty reduction, and environment in the tropical forests. Policy Research Report. Banque Mondiale. Washington. D.C. http://go.worldbank.org/KVK3ZDK510 (26 nov. 2008).

CISDL (Centre for International Sustainable Development Law) et GPPI (Global Public Policy Institute) 2007 A carbon stock approach to creating a positive incentive to reduce emissions from deforestation and forest degradation. Joint submission to the UNFCCC on reducing emissions from deforestation in developing countries. 23 février.

Colfer, C.J.P. et Capistrano, D. (eds.) 2005 The politics of decentralization: Forests, power, and people. Earthscan, Londres, RU.

Colchester, M. 2008 Beyond tenure: Rights-based approaches to peoples and forest areas: Some lessons from the Forest Peoples Programme. FPP and RRI, Moreton-in-Marsh, RU.

Colchester, M. et Ferrari, M. 2007 Making FPIC work: Challenges and prospects for indigenous peoples. FPIC Working Papers, Forest Peoples Program.

Convention internationale sur les droits civils et politiques, GA Res. 2200A (XXI), 21 UN GAOR Supp. (No. 16) at 52, UN Doc. A/6316 (1966); 999 UNTS 171; 6 ILM 368 (1967).

Convention internationale sur les droits économiques, sociaux et culturels, GA Res. 2200A (XXI), 21 UN GAOR Supp. (No. 16) at 49, UN Doc. A/6316 (1966); 993 UNTS 3; 6 ILM 368 (1967).

Convention relative aux peuples indigènes et tribaux dans les pays indépendants (ILO No. 169), 72 ILO Official Bull. 59; 28 ILM 1382 (1989).

Convention sur la diversité biologique, 1760 UNTS 79; 31 ILM 818 (1992).

Convention sur l'élimination de toutes les formes de discrimination à l'égard des femmes, GA Res. 34/180, 34 UN GAOR Supp. (No. 46) at 193, UN Doc. A/34/46; 1249 UNTS 13; 19 ILM 33 (1980).

Corbera, E. 2005 Bringing development into carbon forestry markets: Challenges and outcomes of small-scale carbon forestry activities in Mexico. Dans: Murdiyarso, D. et Herawati, H. (eds.) Carbon Forestry: Who will benefit? p. 42-56. CIFOR, Bogor, Indonésie.

Correa, R. et Moreno, L. 2007 Keeping ITT crude underground: the proposal. Ministère des affaires étrangères, du commerce et de l'intégration, Quito, Ecuador. 
Cosbey, A., Murphy, D., Drexhage, J. et Balint, J. 2006 Making development work in the CDM: Phase II of the Development Dividend Project. IISD, Winnipeg, Canada.

da Fonseca, G.A.B., Rodríguez, C.M., Midgley, G., Busch, J., Hannah, L. et Mittermeier, R.A. 2007 No forest left behind. PLoS Biology 5 (8): 16451646.

Décision 1/CP.13. Plan d'Action de Bali, FCCC/CP/2007/6/Add.1.

Décision 2/CP.13. Réduction des émissions résultant du déboisement dans les pays en développement : Démarches incitatives, FCCC/CP/2007/6/ Add.1.

Déclaration universelle des droits de l'homme, GA Res. 217A (III), U.N. Doc $\mathrm{A} / 810$ at 71 (1948).

DeFries, R., Achard, F., Brown, S., Herold, M., Murdiyarso, D., Schlamadinger, B. et de Sourza Jr., C. 2006 Reducing greenhouse gas emissions from deforestation in developing countries: Considerations for monitoring and measuring. Global Terrestrial Observing System (GTOS), Rome.

DeFries, R., Achard, F., Brown, S., Herold, M., Murdiyarso, D., Schlamadinger B. et de Souza, C. Jr. 2007 Earth observations for estimating greenhouse gas emissions from deforestation in developing countries. Environmental Science and Policy 10 (4): 385-394.

de Jong, B., Bazán, E.E. et Montalvo, S.Q. 2007 Application of the” Climafor " baseline to determine leakage: the case of Scolel Té. Mitigation and Adaptation Strategies for Global Change 12 (6): 1153-1168.

Dutschke, M. 2002 Fractions of permanence - Squaring the cycle of sink carbon accounting. Mitigation and Adaptation Strategies for Global Change 7 (4): 381-402.

Dutschke, M. 2007 CDM forestry and the ultimate objective of the Climate Convention. Mitigation and Adaptation Strategies for Global Change 12 (2): 275-302.

Dutschke, M. 2008 The climate stabilization fund - Global auctioning of emission allowances to help forests and people. Climate 2008/Klima 2008, Scientific Online Climate Conference. www.climate2008.net (25 nov. 2008).

Dutschke, M. et Michaelowa, A. 2006 Development assistance and the CDM - how to interpret" financial additionality'. Environment and Development Economics 11 (2): 235-246.

Dutschke, M. et Wolf, R. 2007 Reducing emissions from deforestation in developing countries. The way forward. GTZ Climate Protection Programme, Eschborn, Allemagne. 29p.

Ebeling, J. et Yasue, M. 2008 Generating carbon finance through avoided deforestation and its potential to create climatic, conservation and human development benefits. Philosophical Transactions of the Royal Society for Biological Sciences B, 363 (1498): 1917-1924. 
ECJRC (Centre commun de Recherche de la Commission Européenne) 2003 The global land cover map for the year 2000. GLC2000 database, Centre commun de Recherche de la Commission Européenne, Bruxelles.

Eggleston, S. 2008 Overview of relevant methodologies in IPCC Guidelines and Good Practice Guidance. Presentation at the UNFCCC workshop on Methodological Issues relating to Reducing Emissions from Deforestation and Forest Degradation in Developing Countries. Tokyo, 24-27 juin.

http://unfccc.int/files/methods_and_science/lulucf/application/pdf/080625_ tokyo_eggleson_ipcc.pdf (25 nov. 2008).

Eliasch J. 2008 Eliasch Review - Climate change: Financing global forests. UK Office of Climate Change www.occ.gov.uk/activities/eliasch.htm (25 nov. 2008).

Enkvist, P.A., Nauclér, T. et Rosander, J. 2007 A cost curve for greenhouse gas reduction. McKinsey Quarterly 2007 (1): 35-45.

Euroactiv 2008 Brussels pushing for forests in global climate deal. Euroactiv, 20 Octobre, Bruxelles. http://www.euractiv.com/en/environment/brusselspushing-forests-global-climate-deal/article-176474 25 nov. 2008).

Fearnside, P.M. 2000 Uncertainty in land use change and forestry sector mitigation options for global warming: Plantation silviculture versus avoided deforestation. Biomass and Bioenergy 18 (6): 457-468.

Fearnside, P.M., Lashof, D.A. et Moura-Costa, P. 2000 Accounting for time in mitigating global warming through land-use change and forestry. Mitigation and Adaptation Strategies for Global Change 5 (3): 239-270.

Fearnside, P.M. 2002 Time preference in global warming calculations: A proposal for a unified index. Ecological Economics 41 (1): 21-31.

Fischlin, A., Midgley, G.F. 2007 Ecosystems, their properties, goods, and services. Dans: Parry, M.L., Canziani, O.F., Palutikof, J.P., van der Linden, P.J. et Hanson, C.E. (eds.) Climate change 2007: Impacts, adaptation and vulnerability. Contribution of Working Group II to the Fourth Assessment Report of the Intergovernmental Panel on Climate Change, 211-272. Cambridge University Press, Cambridge, RU.

Fisher, B., Nakicenovic, N., Alfsen, K., Corfee Morlot, J., de la Chesnaye, F., Hourcade, J-C., Jiang, K., Kainuma, M., La Rovere, E., Matysek, A., Rana, A., Riahi, K., Richels, R., Rose S., van Vuuren, D. et Warren, R. 2007 Issues related to mitigation in the long term context. Dans: Metz, B., Davidson, O.R., Bosch, P.R., Dave, R. et Meyer, L.A. (eds.) Climate change 2007: Mitigation of climate change. Contribution of Working Group III to the Fourth Assessment Report of the Intergovernmental Panel on Climate Change, Cambridge University Press, Cambridge, RU.

Foti, J., de Silva, L., Werksman, J., Shaffer, L., Talbot, J. et McGray, H. 2008 Voice and choice: Opening the door to environmental democracy. World Resources Institute, Washington, D.C.

Gan, J. et McCarl, B. 2007 Measuring transnational leakage of forest conservation. Ecological Economics 64 (2): 423-432. 
Gibbs, H.K., Brown, S., O’Niles, J. et Foley, J.A. 2007 Monitoring and estimating forest carbon stocks: Making REDD a reality. Environmental Resource Letters 2 (2007): 045023 (13pp).

GOFC-GOLD 2008 Reducing greenhouse gas emissions from deforestation and degradation in developing countries: A sourcebook of methods and procedures for monitoring, measuring and reporting, GOFC-GOLD Report version COP 13-2. GOFC-GOLD Project Office, Natural Resources Canada, Alberta, Canada.

Grieg-Gran, M. 2008 The cost of avoiding deforestation. IIED, London: 20. http://www.iied.org/pubs/pdfs/G02290.pdf (25 Nov. 2008).

Hamilton, K., Bayon, R., Turner, G. et Higgins, D. 2007 State of the voluntary carbon markets 2007: Picking up steam. The Ecosystem Marketplace and New Carbon Finance, Washington, D.C.

Hamilton, K., Sjardin, M., Marcello, T. et Xu, G. 2008 Forging a frontier: State of the voluntary carbon markets 2008. Ecosystem Market Place and New Carbon Finance, San Francisco, USA et Londres.

Hansen, M.C., Stehman, S.V., Potapov, P.V., Loveland, T.R., Townshed, J.R.G., DeFries, R.S., Pittman, K.W., Arunarwati, B., Stolle, F., Steininger, M.K., Carroll, M. et DiMiceli, C. 2008 Humid tropical forest clearing from 2000 to 2005 quantified by using multitemporal and multiresolution remotely sensed data. PNAS 105 (27): 9439-9444.

Hardcastle, P.D. et Baird, D. 2008 Capability and cost assessment of the major forest nations to measure and monitor their forest carbon. Office of Climate Change. LTS International, Penicuick, RU. http://www.occ.gov. uk/activities/eliasch.htm (25 nov. 2008).

Hare, B. et Macey, K. 2007 Tropical deforestation emission reduction mechanism (TDERM): A discussion paper. Greenpeace International, Amsterdam.52p. http://www.greenpeace.org/raw/content/international/ press/reports/TDERM-full.pdf (25 nov. 2008).

Hoare, A., Legge, T., Nussbaum, R. et Saunders, J. 2008 Estimating the cost of building capacity in rainforest nations to allow them to participate in a global REDD mechanism. Chatham House and ProForest, RU. (25 nov. 2008). http://www.occ.gov.uk/activities/eliasch/Chatham_House_cost_ of_building_capacity.pdf

Hughes, R. et Flintan, F. 2001 Integrating conservation and development experience: a review and bibliography of the ICDP literature. International Institute for Environment and Development, Londres. 24p. http://www. ucc.ie/famine/GCD/ICDP_sec.pdf (25 nov. 2008).

INPE 2004 Monitoramento ambiental da Amazonia por satelite. Brazilian Institute for Space Research. http://www.obt.inpe.br/prodes/ (25 nov. 2008).

GIEC (Groupe d'experts intergouvernemental sur l'évolution du climat) 2003 Guide de bonnes pratiques sur l'utilisation des terres, les changements d'affectation et la foresterie, préparé par the National Greenhouse Gas Inventories Programme. Eggleston, H.S., Buendia, L., Miwa, K., Ngara, T. 
et Tanabe, K. (eds.). Institute for Global Environmental Strategies (IGES), Kanagawa, Japon.

GIEC 2003b Guide de bonnes pratiques pour l'utilisation des terres, les changements d'affectation et la foresterie (GPG-LULUCF). Penman, J., Gytarsky, M., Krug, T., Kruger, D., Pipatti, R., Buendia, L., Miwa, K., Ngara, T., Tanabe, K. et Wagner, F. (eds.), IPCC-IGES, Kanagawa.

http://www.ipcc-nggip.iges.or.jp/public/gpglulucf/gpglulucf_contents.html (25 nov. 2008).

GIEC 2006 Lignes directrices pour les inventaires nationaux des gaz à effet de serre, prepared by the National Greenhouse Gas Inventories Programme. Eggleston, H.S., Buendia, L., Miwa, K., Ngara, T. et Tanabe, K. (eds.). Institute for Global Environmental Strategies (IGES), Japon.

http://www.ipcc-nggip.iges.or.jp/public/2006gl/index.html (25 nov. 2008).

GIEC (Groupe d'experts intergouvernemental sur l'évolution du climat) 2006 Lignes directrices pour les inventaires nationaux des gaz à effet de serre - volume 4: Agriculture, utilisation des terres et foresterie (GL-AFOLU). http://www.ipcc-nggip.iges.or.jp/public/2006gl/vol4.html (25 nov. 2008).

Iskandar, H., Snook, L., Toma, T., MacDicken, K. et Kanninen, M. 2006 A comparison of damage due to logging under different forms of resource access in East Kalimantan, Indonesia. Forest Ecology and Management 237 (1-3): 83-93.

Jakeman, G. et Fisher, B.S. 2006 Benefits of multi-gas mitigation: An application of the Global Trade and Environment Model (GTEM), multigas mitigation and climate policy. The Energy Journal 27 (3): 323-342.

Kanowski, J.J., Catterall, C. et Wardell-Johnson, G.W. 2005 Consequences of broadscale timber plantations for biodiversity in cleared rainforest landscapes of tropical and subtropical Australia. Forest Ecology and Management 208 (1-3): 359-372.

Karousakis, K. 2007 Incentives to reducing emissions from deforestation: Lessons learned from Costa Rica and Mexico. OCDE, Paris. 50p.

Khan, M. 2006 State failure in developing countries and strategies of institutional reform. http://www.gdnet.org/pdf2/online_journals/cerdi/ issue2_3/Khan_paper1.pdf (25 nov. 2008).

Kindermann, G., Obersteiner, M., Sohngen, B., Sathaye, J., Andrasko, K., Ramesteiner, E., Schlamadinger, B., Wunder, S. et Beach, R. 2008 Global cost estimates of reducing carbon emissions through avoided deforestation. Proceedings of the National Academy of Sciences 105 (30): 1030210307.

Korhonen, L., Korhonen, K.T., Rautiainen, M. et Stenberg, P. 2006 Estimation of forest canopy cover: A comparison of field measurement techniques. Silva Fennica 40 (4): 577-588.

www.metla.fi/silvafennica/full/sf40/sf404577.pdf (25 nov. 2008).

Kurosawa, A. 2006 Multi-gas mitigation: An economic analysis using the GRAPE model. The Energy Journal 27 (3): 275-288. 
Lambin, E.F., Geist, H.J. et Lepers, E. 2003 Dynamics of land-use and land-cover change in tropical regions. Annual Review of Environmental Resources 28: 205-241.

Larson, A. et Ribot, J. 2007 The poverty of forestry policy: Double standards on an uneven playing field. Sustainability Science 2 (2): 189-204.

Leach, P. 2008 Carbon sunk? The potential impacts of avoided deforestation credits on emissions trading mechanisms. The Rainforest Foundation, Londres.

http://www.rainforestfoundationuk.org/Carbon_Sunk (25 nov. 2008).

Lecocq, F. et Chomitz, K.M. 2001 Optimal use of carbon sequestration in a global climate change strategy: Is there a wooden bridge to a clean energy future? World Bank Development Research Group Infrastructure and Environment, Washington, D.C.

Marklund, L.G. et Schoene, D. 2006 Global assessment of growing stock, biomass and carbon stock. Forest Resources Assessment Programme Working paper 106/E, Rome.

Massai, L. 2007 European Climate Policy Dossier. T.M.C. Asser Institute, La Haye, Pays-Bas. 57p.

Mather, A. 1992 The Forest Transition. Area 24 (4): 367-379.

M-Co Consulting 2008 Review and assessment of options for reducing emissions from deforestation in developing countries. Gouvernement de la Nouvelle-Zélande, Ministère de l'agriculture et de la foresterie, Wellington.

Meijaard, E., Sheil, D., Nasi, R., Augeri, D., Rosenbaum, B., Iskandar, D., Setyawati, T., Lammertink, M., Rachmatika, I., Wong, A., Soehartono, T., Stanley, S. et O’Brien, T. 2005 Life after logging: Reconciling wildlife conservation and production forestry in Indonesian Borneo. CIFOR, Bogor, Indonésie.

http://www.cifor.cgiar.org/publications/pdf_files/books/BMeijaard0501E0. pdf (25 nov. 2008).

Miles, L., Kapos, V., Lysenko, I. et Campbell, A. 2008 Mapping vulnerability of tropical forest to conversion, and resulting $\mathrm{CO}_{2}$ emissions: A rapid assessment for the Eliasch review. UNEP World Conservation Monitoring Centre.

http://www.occ.gov.uk/activities/eliasch/UNEP_WCMC_mapping_ vulnerability_of_tropical_forest(1).pdf (25 nov. 2008).

Mollicone, D., Achard, F., Federici, S., Eva, H.D., Grassi, G., Belward, A., Raes, F., Seufert, G., Stibig, H.J., Matteucci, G. et Schulze E.D. 2007 An incentive mechanism for reducing emissions from conversion of intact to non-intact forests. Climate Change 83 (4): 477-493.

Motel, P.C., Pirard, R. et Combes, J.L. 2008 A methodology to estimate impacts of domestic policies on deforestation: Compensated successful efforts for" avoided deforestation "(REDD). Ecological Economics (en préparation). 
Moura-Costa, P. et Wilson, C. 2000 An equivalence factor between $\mathrm{CO}_{2}$ avoided emissions and sequestration: Description and applications in forestry. Mitigation and Adaptation Strategies for Global Change 5 (1): 51-60.

Muller, A. 2007 How to make the clean development mechanism sustainable - the potential of rent extraction. Energy Policy 35 (6): 3203-3212.

Murphy, P.G. et Lugo, A.E. 1986 Ecology of tropical dry forest. Annual Review of Ecology and Systematics 17: 67-68.

Murray, B.C. 2008 Leakage from an avoided deforestation compensation policy: Concepts, empirical evidence, and corrective policy options. Nicholas Institute for Environmental Policy Solutions, Duke University, Durham, North Carolina, USA. 32p.

Nepstad, D. 2007 The Amazon's vicious cycles: Drought and fire in the greenhouse. WWF Report. http://assets.panda.org/downloads/amazonas_ eng_04_12b_web.pdf (25 nov. 2008).

Nepstad, D., Soares-Filho, B., Merry, F., Moutinho, P., Oliveira Rodrigues, H., Bowman, M., Schwartzman, S., Almeida, O.et Rivero, S. 2007 The costs and benefits of reducing deforestation in the Brazilian Amazon. The Woods Hole Research Center, Woods Hole, Massachusetts, USA.

Obersteiner, M., Azar, C., Kauppi, P., Möllersten, K., Moreira, J., Nilsson, S., Read, P., Riahi, K., Schlamadinger, B., Yamagata, Y., Yan, J. et van Ypersele, J.-P. 2001 Managing climate risk. Science 294 (5543): 786-787.

OCDE (Organisation de coopération et de développement économiques) 2005 Déclaration de Paris sur l'efficacité de l'aide : Appropriation, harmonisation, alignement, résultats et responsabilité mutuelle. OCDE, Paris. www.oecd. org/dataoecd/11/41/34428351.pdf (25 nov. 2008).

Ogonowski, M., Helme, N., Movius, D. et Schmidt, J. 2007 Reducing emissions from deforestation and degradation: The dual markets approach. International Future Action Dialogue. Center for Clean Air Policy, Washington, D.C.

Olander, L.P., Gibbs, H.K., Steininger, M., Swenson, J.J. et Murray, B.C. 2008 Reference scenarios for deforestation and forest degradation in support of REDD: A review of data and methods. Environmental Research Letters 3 (2008): 025011. http://www.iop.org/EJ/article/1748-9326/3/2/025011/ erl8_2_025011.pdf (25 nov. 2008).

Pearce, F. 2007 Save the climate by saving the forest. New Scientist, 22 mars 2008.

Pearson, T., Harris, N., Shock, D., Pandey, D. et Brown, S. 2008 Estimation of carbon stocks. Chapitre 4 Dans: GOFC-GOLD. Reducing greenhouse gas emissions from deforestation and degradation in developing countries: A sourcebook of methods and procedures for monitoring, measuring and reporting, GOFC-GOLD Report version COP13-2, GOFC-GOLD Project Office, Natural Resources Canada, Alberta, Canada. 
Pedroni, L., Streck, C., Estrada, M. et Dutschke, M. 2007 The” Nested Approach': A flexible mechanism to reduce emissions from deforestation. CATIE, Turrialba, Costa Rica.

Penman, J., Gytarsky, M., Hiraishi, T., Krug, T., Kruger, D., Pipatti, R., Buendia, L., Miwa, K., Ngara, T., Tanabe, K. et Wagner, F. 2003 Good practice guidance for land use, land-use change and forestry. IPCC National Greenhouse Gas Inventories Programme and Institute for Global Environmental Strategies (IGES) (Groupe d'experts intergouvernemental sur l'évolution du climat), Kanagawa, Japon.

http://www.ipcc-nggip.iges.or.jp/public/gpglulucf/gpglulucf_contents.htm (25 nov. 2008).

Penman, J., Gytarsky, M., Krug, T., Kruger, D., Pipatti, R., Buendia, L., Miwa, K., Ngara, T., Tanabe, K. et Wagner, F. (eds.) 2003a Definitions and methodologies for inventory emissions from direct human-induced degradation of forests and devegetation of other vegetation types. IPCCIGES, Kanagawa, Japon.

Penman, J. 2008 An exploration by the EU on methodological issues relating to reducing emissions from forest degradation in developing countries. UNFCCC Informal Meeting of Experts, Bonn, Allemagne, 20-21 Octobre 2008. http://unfccc.int/methods_science/redd/items/4579.php (25 nov. 2008).

Peskett, L., Huberman, D., Bowen-Jones, E., Edwards, G. et Brown, J. 2008 Making REDD work for the poor. IUCN/ODI for the Poverty and Environment Partnership, Gland.

Petley, S. 2007 Forest backed securities: Alternative finance for tropical natural forest. Presentation to the Asia-Pacific Tropical Forest Investment Forum, Août, 2007.

www.itto.or.jp/live/Live_Server/3289/PetleyITTOBangkokREV.JG.pdf nov. 2008).

Piris-Cabezas, P. et Keohane, N. 2008 Reducing emissions from deforestation and degradation in developing countries (REDD): Implications for the carbon market. Environmental Defense Fund, Washington, D.C. 13p.

http://www.climaedesmatamento.org.br/files/general/EDF_Analysis_of_ REDD_in_the_carbon_market_061808.pdf (25 Nov. 2008).

Rao, S. et Riahi, K. 2006 The role of non- $\mathrm{CO}_{2}$ greenhouse gases in climate change mitigation: Long-term scenarios for the $21^{\text {st }}$ Century, multi-gas mitigation and climate policy. Energy Journal 27 (3): 177-200.

Riahi, K., Grubler, A. et Nakicenovic, N. 2006 Scenarios of long-term socioeconomic and environmental development under climate stabilisation. Technological Forecasting and Change 74: 8-9.

Rights and Resources Initiative, 2008. Foundations for effectiveness. Policy brief prepared by RRI and RFN in preparation for the International Conference on Rights, Forests and Climate Change, Oslo, 15-17 Octobre, 2008.

Rio Declaration, UN Doc. A/CONF.151/26 (Vol. I); 31 ILM 874 (1992). 
Robertson, N. et Wunder, S. 2005 Fresh tracks in the forest: Assessing incipient payments for environmental services initiatives in Bolivia. CIFOR, Bogor, Indonésie. $137 \mathrm{p}$.

http://www.cifor.cgiar.org/pes/publications/pdf_files/BRobertson0501.pdf (25 nov. 2008).

Rose, S., Helal, A., Eickhout, B., Fisher, B., Kurosawa, A., Rao, S., Riahi, K. et van Vuuren, D. 2007 Land in climate stabilization modeling: Initial observations. Energy Modeling Forum Report, Stanford University, Stanford, California, USA.

Roy, D.P., Jin, Y., Lewis, P.E. et Justice, C.O. 2005 Prototyping a global algorithm for systematic fire-affected area mapping using MODIS timeseries data. Remote Sensing of Environment 97 (2): 137-162.

Rudel, T.K., Coomes, O.T, Moran, E., Achard, F., Angelsen, A., Jianchu Xu et Lambin, E. 2005 Forest transitions: Towards a global understanding of land use change. Global Environmental Change 15 (1): 23-31.

Santilli, M., Moutinho, P., Schwartzman, S., Nepstad, D., Curran, L. et Nobre, C. 2005 Tropical deforestation and the Kyoto Protocol. Climatic Change 71 (3): 267-276.

Sathaye, J. et Andrasko, K. 2007 Special issue on estimation of baselines and leakage in carbon mitigation forestry projects. Mitigation and Adaptation Strategies for Global Change 12 (6): 963-970.

Schelhas, J. et Sanchez-Azofeifa, G.A. 2006 Post-frontier forest change adjacent to Braulio Carrillo National Park, Costa Rica. Human Ecology 34 (3): 407-431.

Schlamadinger, B., Ciccarese, L., Dutschke, M., Fearnside, P.M., Brown, S. et Murdiyarso, D. 2005 Should we include avoidance of deforestation in the international response to climate change? Dans: Carbon forestry: Who will benefit? Murdiyarso, D. et Herawati, H. (eds.) CIFOR, Bogor, Indonésie.

Schlamadinger, B. et Johns, T. 2006 Reducing emissions from deforestation and forest degradation: Latest developments. Climate Change Mitigation Measures in the Agro-Forestry Sector and Biodiversity Futures, ICTP, Trieste, Italie.

Schlamadinger, B., Bird, N., Johns, T., Brown, S., Canadell, J., Ciccarese, L., Dutschke, M., Fiedler, J., Fischlin, A., Fearnside, P., Forner, C., Freibauer, A., Frumhoff, P., Hoehne, N., Kirschbaum, M.U.F., Labat, A., Michaelowa, A., Montanarella, L., Moutinho, P. Murdiyarso, D., Pena, N., Pingoud, K., Rakonczay, Z., Rametsteiner, E., Rock, J., Sanz, M.J., Schneider, U.A., Shvidenko, A., Skutsch, M., Smith, P., Somogyi, Z., Trines, E., Ward, M. et Yamagata, Y. 2007 A synopsis of land use, land-use change and forestry (LULUCF) under the Kyoto Protocol and Marrakech Accords. Environmental Science and Policy 10 (4): 271-282.

Scholz, I. et Schmidt, L. 2008 Reducing emissions from deforestation and forest degradation in developing countries: Meeting the main challenges ahead. German Development Institute (DIE) Briefing Paper (version préliminaire). 
http:/www.illegal-logging.info/uploads/GermanDevInstREDD.pdf (25 nov. 2008).

Schwarze, R., Niles, J.O. et Olander, J. 2002 Understanding and managing leakage in forest-based greenhouse gas mitigation projects. TNC, Arlington, Virginia, USA.

Seymour, F. (prochainement) Forests, climate change, and human rights: Managing risks and trade-offs. Dans: Humphreys, S. (ed.) Human rights and climate change. Cambridge University Press, Cambridge, RU.

Skutsch, M. et Trines, E. 2008 Operationalising reduced degradation within REDD. Policy Paper No.2: Kyoto: Think Globally Act Locally project.

http://www.communitycarbonforestry.org/resources_Pub08.htm (25 nov. 2008).

Sohngen, B. et Brown, S. 2004 Measuring leakage from carbon projects in open economies: a stop timber harvesting project in Bolivia as a case study. Canadian Journal of Forestry Research 34 (4): 829-839.

Stern, N. 2006 Stern Review: The economics of climate change. Cambridge University Press, Cambridge, RU.

Stern, N. 2008 Key elements of a global deal on climate change. London School of Economics and Political Science, Londres. 56p.

Strassburg, B., Turner, K., Fisher, B., Schaeffer, R. et Lovett, A. 2008 An empirically-derived mechanism of combined incentives to reduce emissions from deforestation. Dans: CSERGE Working Paper ECM 08-01. Centre for Social and Economic Research on the Global Environment (CSERGE), University of East Anglia, Norwich, RU.

Subak, S. 2003 Replacing carbon lost from forests: an assessment of insurance, reserves, and expiring credits. Climate Policy 3 (2): 107-122.

Sunderlin, W., Hatcher, J. et Liddle, M. 2008 From exclusion to ownership? Challenge and opportunities in advancing forest tenure reform. Rights and Resources Initiative, Washington, D.C.

http://www.rightsandresources.org/documents/index.php?pubID=736 nov. 2008).

Tavoni, M., Sohngen, B. et Bosetti, V. 2007 Forestry and the carbon market response to stabilize climate. Energy Policy 35 (11): 5346-5353.

Terrestrial Carbon Group 2008 How to include terrestrial carbon in developing countries in the overall climate change solution. Draft, 8 août.

Ramsar Convention: Convention relative aux zones humides d'importance internationale particulièrement comme habitats des oiseaux d'eau. Ramsar (Iran), 2 février 1971. UN Treaty Series No. 14583.

Trines, E., Höhne, N., Jung, M., Skutsch, M., Petsonk, A., Silva-Chavez, G., Smith, P., Nabuurs, G., Verweij, P. et Schlamadinger, B. 2006 Integrating agriculture, forestry and other land use in future climate regimes. Methodological issues and policy options. Netherlands Environmental Assessment Agency, Bilthoven, Pays-Bas. 
Tutin, C.E.G. et Fernandez, M. 1985 Foods consumed by sympatric populations of Gorilla g. gorilla and Pan t. troglodytes in Gabon: Some preliminary data. International Journal of Primatology 6 (1): 27-43.

Tuvalu (Government of) 2007 Submission from Tuvalu Dans: Reducing emissions from deforestation in developing countries: Approaches to stimulate action. Subsidiary Body for Scientific and Technological Advice, Twenty-seventh session, Bali, 3-11 décembre 2007.

http://unfccc.int/resource/docs/2007/sbsta/eng/misc14a03.pdf (25 nov. 2008).

Underdal, A. 2002 One question, two answers. Dans: Miles, E.L., Underdal, A., Andersen, S., Wettestad, J., Skærseth, J.B. et Carlin, E.M. (eds.) Environmental regime effectiveness. Confronting theory with evidence. MIT Press, Cambridge, Massachusetts, USA.

Nations Unies: Déclaration sur les droits des peuples autochtones, GA Res. 61/295, U.N. Doc. A/RES/61/295 (13 septembre 2007), 46 I.L.M. 1013 (2007).

United Nations Forum on Forests (UNFF) Non-legally binding instrument on all types of forests, E/2007/42.

Nations Unies: Convention des Nations Unies sur l'accès à l'information, la participation du public et l'accès à la justice en matière d'environnement, 2161 UNTS 447; 38 ILM 517 (1999).

Nations Unies : Convention des Nations Unies sur la lutte contre la désertification, 1954 UNTS 3; 33 ILM 1328 (1994).

UNFCCC 2007 Investment and financial flows to address climate change. UNFCCC, Bonn, Allemagne.

CCNUCC 2007c Organe subsidiaire de conseil scientifique et technologique, Vingt-septième Session, Bali, Indonésie, 3-11 décembre 2007.

UNFCCC 2008a Views on outstanding methodological issues related to policy approaches and positive incentives to reduce emissions from deforestation and forest degradation in developing countries. Advanced version. SBSTA Misc. for its $28^{\text {th }}$ session. Bonn, Allemagne, 4-13 juin 2008.

UNFCCC 2008b Informal meeting of experts on methodological issues relating to reducing emissions from forest degradation in developing countries. Bonn, Allemagne, 20-21 octobre 2008.

Ward, M., Strect, C., Winkler, H., Jung, M., Hagemann, M., Höhne, N. et O'Sullivan, R. 2008 The role of sector no-lose targets in scaling up finance for climate change mitigation activities in developing countries. International Climate Division, Dept. of Environment, Food and Rural Affairs (DERFA), RU.

Watson, R.T., Intergovernmental Panel on Climate Change, Noble, I.R., Bolin, B. 2000 Land use, land-use change, and forestry: A special report of the Intergovernmental Panel on Climate Change. Cambridge University Press, Cambridge, RU. 377p.

Wertz-Kanounnikoff, S. 2008 Cost-effective methods for monitoring forest cover changes and associated $\mathrm{CO}_{2}$ emissions for REDD. CIFOR, Bogor, 
Indonesia, International Institute for Environment and Development (IIED), Londres et World Resources Institute (WRI), Washington, D.C.

WHRC (Woods Hole Research Center) et IPAM (Instituto de Pesquisa Ambiental da Amazonia) 2008 How to distribute REDD funds across countries? A stock-flow mechanism. Joint submission to the UNFCCC regarding AWG-LCA (FCCC/AWGLCA/2008/L.7), 30 septembre 2008.

Winrock. 2002 Analysis of leakage, baselines, and carbon benefits for the Noel Kempff Climate Action Project. 45. Ecosecurities Ltd., Sylvan Acres, Geographic Modeling Services.

Wittemyer, G., Elsen, P., Bean, W.T., Coleman, A., Burton, O. et Brashares, J.S. 2008 Accelerated human population growth at protected area edges. Science 321 (5885): 123-126.

Wong, J. et Dutschke, M. 2003 Can permanence be insured? Consideration of some technical and practical issues of insuring carbon credits from afforestation and reforestation. HWWA Discussion Paper 235. 17p.

Wu, J.J. 2000 Slippage effects of the Conservation Reserve Program. American Journal of Agricultural Economics 82 (4): 979-992. 

La REDD (réduction des émissions dues à la déforestation et à la dégradation des forêts) est fondée sur une idée simple: compenser les pays en développement pour qu'ils réduisent leurs émissions de $\mathrm{CO}_{2}$ en provenance du secteur forestier. Cependant, la conception et la mise en œuvre d'un système REDD suscitent immédiatement nombre de questions difficiles: Comment surveiller (mesurer), en rendre compte et vérifier les réductions d'émissions? Comment financer la REDD? Faut-il effectuer les paiements de compensation au titre des pays, des projets ou à ces deux titres? Comment fixer les niveaux de référence? Comment rendre compte de la non permanence et des fuites? Comment obtenir des retombées positives et éviter les effets nocifs?

Faire progresser la REDD : Enjeux, options et répercussions est un ouvrage qui ne cherche pas à apporter des réponses définitives. L'objectif en est plutôt de présenter un certain nombre d'options pour édifier au niveau mondial un système de réduction des émissions (liées au déboisement et à la dégradation des forêts) et d'en évaluer les répercussions sur la base du critère des $3 \mathrm{E}$ - efficacité de cette réduction, efficience des coûts (ou rentabilité), équité et retombées positives. Quiconque participe et s'intéresse au débat sur la REDD et aux négociations y afférentes peut tirer profit de cette présentation claire et concise concernant les enjeux clé de la réduction des émissions dues au déboisement et à la dégradation des forêts.

Co-auteurs : A. Angelsen, S. Atmadja, D. Brown, J. Brown, M. Dutschke, M. Guariguata, P. Guizol, M. Kanninen, R. Lubowski,

C. Luttrell, D. Murdiyarso, L. Peskett, F. Seymour, M. Skutsch,

O. Stella Martins, C. Streck, L.V. Verchot, P. Verweij,

S. Wertz-Kanounnikoff et S. Wunder

ISBN 978-6-02-869305-9

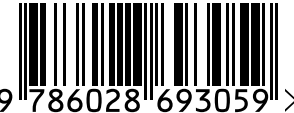

$9786028^{11693059^{\prime}>}$

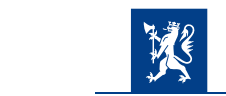

NORWEGIAN MINISTRY OF THE ENVIRONMENT

ENVIRONMENT
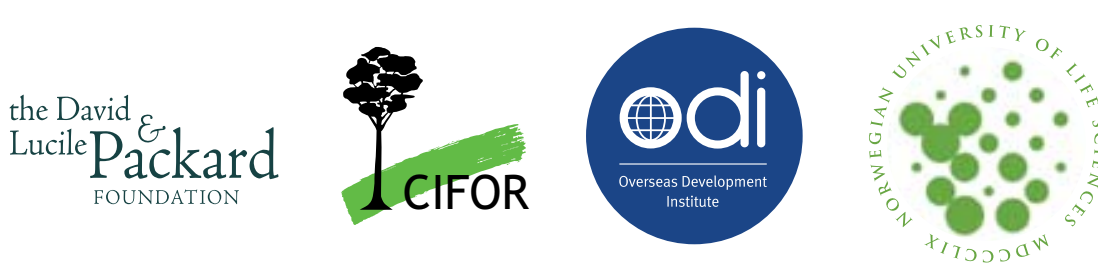

e

CLIMATEFOCUS
ENVIRONMENTAL DEFENSE FUND finding the ways that work 